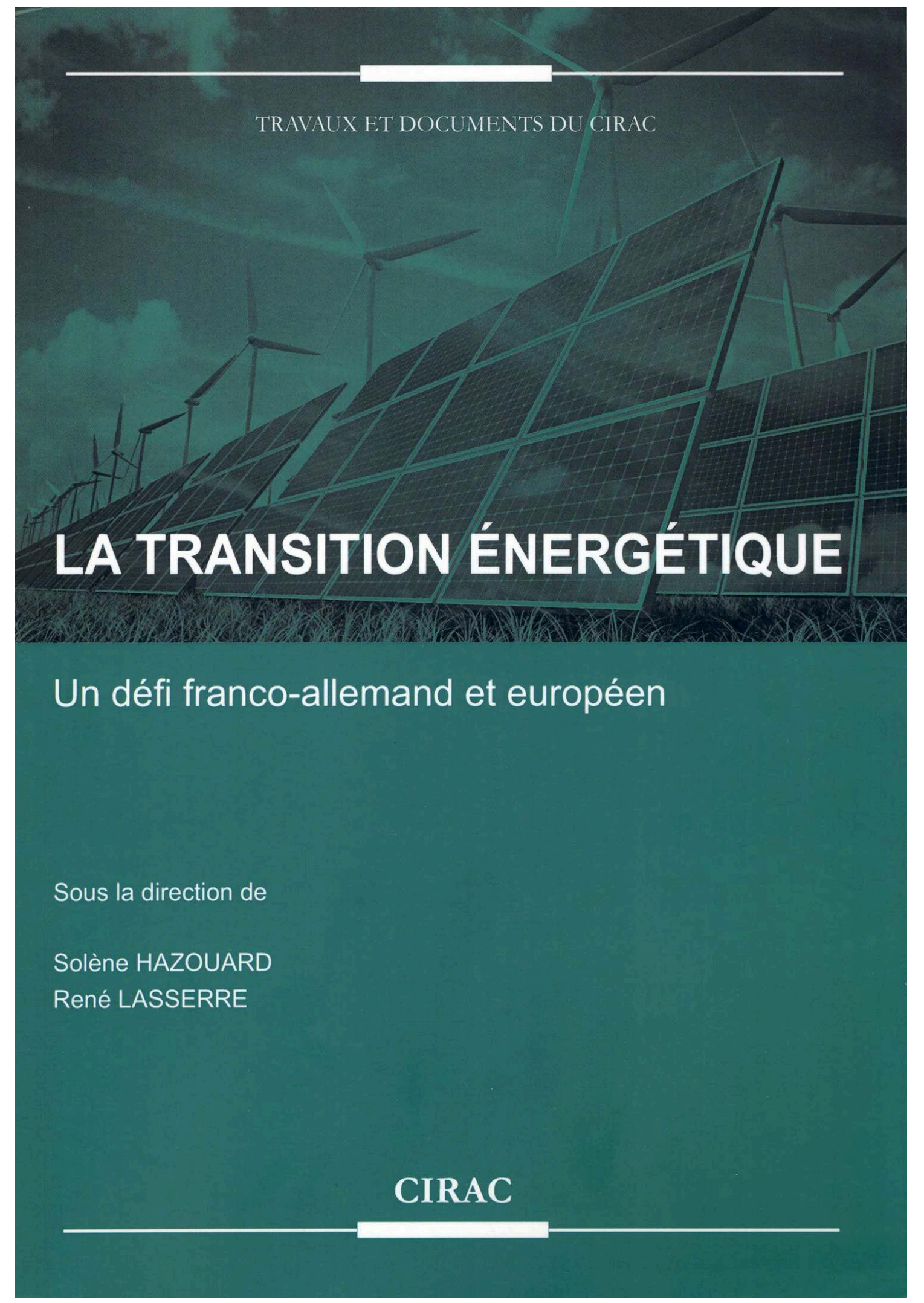




\section{La transition énergétique}

Un défi franco-allemand et européen

Solène Hazouard et René Lasserre (dir.)

DOI : 10.4000/books.cirac.999

Éditeur : CIRAC

Lieu d'édition : Cergy-Pontoise

Année d'édition : 2017

Date de mise en ligne : 21 décembre 2018

Collection : Travaux et documents du CIRAC

EAN électronique : 9782905518729

\section{- OpenEdition}

\section{Books}

https://books.openedition.org

\section{Édition imprimée}

EAN (Édition imprimée) : 9782905518507

Nombre de pages : 270

\section{Référence électronique}

HAZOUARD, Solène (dir.) ; LASSERRE, René (dir.). La transition énergétique : Un défi franco-allemand et européen. Nouvelle édition [en ligne]. Cergy-Pontoise : CIRAC, 2017 (généré le 08 décembre 2022)

Disponible sur Internet : <http://books.openedition.org/cirac/999>. ISBN : 9782905518729 . DOI

https://doi.org/10.4000/books.cirac.999.

(c) CIRAC, 2017

Licence OpenEdition Books 


\section{RÉSUMÉS}

Alors que débute la mise en œuvre des objectifs climatiques établis lors de la COP21 fin 2015 à Paris, voici rassemblées dans un même volume les contributions de deux journées d'étude et d'un colloque consacrés aux questions énergétiques et climatiques en France, en Allemagne et en Europe.

Cet ouvrage se propose dans un premier temps de replacer les stratégies de transition énergétique française et allemande actuelles dans le contexte des politiques menées ces dernières décennies. L'analyse porte ensuite sur des exemples significatifs de coopération francoallemande à différents niveaux décisionnels, avant de se focaliser sur les transformations structurelles du marché de l'électricité. Une cinquième partie est dédiée aux enjeux environnementaux, et notamment à l'impact de la COP21 pour l'Europe. Enfin, l'examen des stratégies énergétiques du Royaume-Uni, de la Suède et de la Pologne met en lumière le long chemin qu'il reste à parcourir avant de pouvoir réellement compter sur une politique européenne de l'énergie.

\section{SOLĖNE HAZOUARD (DIR.)}

Ingénieur d'études au Centre d'information et de recherche sur l'Allemagne contemporaine (CIRAC), Cergy-Pontoise

\section{RENÉ LASSERRE (DIR.)}

Directeur du Centre d'information et de recherche sur l'Allemagne contemporaine (CIRAC) et professeur émérite à l'université de Cergy-Pontoise 


\title{
LA TRANSITION ÉNERGÉTIQUE
}

Un défi franco-allemand et européen

\author{
Sous la direction de \\ Solène HAZOUARD, René LASSERRE
}

\section{CIRAC}




\section{Travaux et documents du CIRAC}

Collection dirigée par René Lasserre

Ouvrage publié en collaboration avec l'Institut allemand de politique étrangère (Deutsche Gesellschaft für Auswärtige Politik, DGAP), avec le concours du Centre interdisciplinaire d'études et de recherches sur l'Allemagne (CIERA), du centre de recherche AGORA de l'université de Cergy-Pontoise et de l'Office franco-allemand pour la transition énergétique (OFATE).

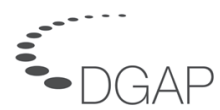

Deutsche Gesellschaft für Auswärtige Politik

German Council on Foreign Relations

\section{ciera AGORA} SET DE RECHERCHES
SUR L'ALLEMAGNE

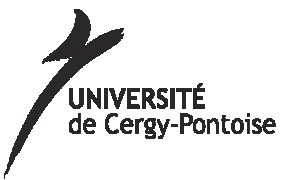

office franco-allemand pour la transition énergétique Deutsch-französisches Büro für die Energiewende

Les contributions publiées dans cet ouvrage engagent uniquement la responsabilité de leurs auteurs.

Illustration de couverture : (C) Flickr - TAFE SA TONSLEY. (https://www.flickr.com/photos/99206485@N02/12648929455/)

(C) CIRAC, 2017

http://www.cirac.u-cergy.fr

CIRAC, c/o Université de Cergy-Pontoise

33, boulevard du Port - 95011 CERGY-PONTOISE CEDEX

ISBN : 978-2-905518-50-7 


\section{LA TRANSITION ÉNERGÉTIQUE \\ UN DÉFI FRANCO-ALLEMAND ET EUROPÉEN}

TABLE DES MATIÈRES

REMERCIEMENTS .

\section{INTRODUCTION}

Transition énergétique et Energiewende : vers une politique européenne de l'énergie?

Solène Hazouard, René Lasserre.

I - Les politiques Énergétiques en France et en Allemagne :

ÉLÉMENTS HISTORIQUES

La France et sa politique de l'énergie (des années 1970 à aujourd'hui)

Alain Beltran.

De la reconstruction à l'Energiewende.

Continuité et changement dans les politiques énergétiques allemandes

Stefan C. Aykut.

II - LA TRANSITION ÉNERGÉTIQUE ET L'ENERGIEWENDE.

Comparatif des politiques énergétiques :

les voies française et allemande pour la transformation du système énergétique

Severin Fischer.

Le tournant énergétique allemand

Michel Cruciani..

Thèses essentielles sur les phases de transformation du système énergétique :

réflexions sur le tournant énergétique en Allemagne

Manfred Fischedick

Quelles solutions pour financer la transition énergétique en France ?

Romain Morel, Hadrien Hainaut, lan Cochran .

Comment financer la transition énergétique en Allemagne ?

Ulrich Benterbusch.

III - EXEMPLES DE COOPÉRATION FRANCO-ALLEMANDE

Exposé introductif :

Le couple franco-allemand : moteur de la transition énergétique ?

Jean-Claude Perraudin .... 
TANDEM : coopération franco-allemande pour la transition énergétique locale Anne Turfin.

Protection du climat, énergies renouvelables et efficacité énergétique dans la Région métropolitaine trinationale du Rhin supérieur Jürgen Oser

Les défis de l'Office franco-allemand pour la transition énergétique

Sven Rösner

IV - DÉFIS ET TRANSFORMATIONS DU MARCHÉ DE L'ÉLECTRICITÉ

Compétitivité : la transition énergétique menace-t-elle nos sites de production ? Joachim Lang

Conséquences de la transition énergétique sur le prix pour le consommateur

Christophe Leininger.....

Le rôle des réseaux dans la réussite de la transition énergétique

Hervé Mignon

Transition énergétique en RFA : impact sur les réseaux électriques en Allemagne

Ulrike Hansen.

Enjeux de la transformation des systèmes électriques

allemand et français à l'horizon 2030

Dimitri Pescia

Le marché européen de l'électricité :

un pilier de la sécurité d'approvisionnement

Wolfram Vogel.

V - LA COP21 ET LA POLITIQUE EUROPÉENNE DE L'ÉNERGIE

Protection du climat et transition énergétique

Berthold Goeke

Quelle contribution la politique énergétique peut-elle apporter au succès de la COP21 ?

Stéphane Reiche.

COP21 : quels enjeux pour l'Europe?

Michel Colombier

La politique énergétique et climatique de l'UE après la COP21

Christian Egenhofer

Le pentagone du marché de l'électricité : une conception pragmatique du marché de l'électricité pour la transition énergétique européenne

Philipp Litz. 
Exposé introductif :

Transition(s) énergétique(s) en Europe : unis dans la diversité ?

Thomas Pellerin-Carlin......

La politique énergétique du Royaume-Uni : orientations passées et futures

Joseph Dutton.....

La politique énergétique d'autres pays d'Europe : le cas de la Suède

Thomas B. Johansson

Les choix énergétiques d'autres pays européens : la Pologne

Marek Wąsiński..... 



\section{REMERCIEMENTS}

Cet ouvrage collectif constitue la synthèse des travaux de deux journées d'étude et d'un colloque, organisés en 2015 et 2016 dans le cadre du projet Transitions énergétiques en Allemagne et en France dans le contexte de la définition d'une politique européenne de l'énergie. Mené par le Centre d'information et de recherche sur l'Allemagne contemporaine (CIRAC), en partenariat avec l'Institut allemand de politique étrangère (Deutsche Gesellschaft für Auswärtige Politik, DGAP), ce projet s'inscrit dans le cadre des programmes de formation-recherche soutenus par le Centre interdisciplinaire d'études et de recherches sur l'Allemagne (CIERA). Il avait pour objet de comparer les politiques actuelles visant à une refonte des bouquets énergétiques français et allemands, dans le contexte des objectifs du paquet énergie-climat de l'Union européenne. Au-delà de la présentation des politiques de transition énergétique française et allemande et de l'évolution binationale des mix énergétiques au cours de ces dernières décennies, le projet s'est proposé d'étudier les défis posés aux responsables politiques, aux autorités de régulation de l'énergie, aux acteurs en charge de la production, du transport et de la distribution d'électricité des deux pays ainsi qu'aux chercheurs impliqués dans ces problématiques de part et d'autre du Rhin. Il s'agissait en outre de mettre en lumière les coopérations et les échanges franco-allemands dans le contexte de la définition d'une politique communautaire de l'énergie et de s'intéresser aux choix énergétiques d'autres pays européens comme le Royaume-Uni, la Suède et la Pologne.

Pour mener à bien ce projet, les organisateurs ont pu bénéficier, en majeure partie, du concours financier apporté par le CIERA, auquel s'est associé le centre de recherche AGORA de l'université de Cergy-Pontoise et l'Office franco-allemand pour la transition énergétique (OFATE). Nous remercions vivement les auteurs, issus de divers pays et de différents horizons scientifiques ou professionnels, pour leur contribution à cet ouvrage, Marie-Céline Georg, qui a assuré la traduction de leurs travaux, ainsi que Claire Demesmay, directrice du programme Relations franco-allemandes à la DGAP, pour sa participation aux échanges avec les auteurs.

Nous tenons également à remercier l'équipe du CIRAC pour son concours, en particulier Isabelle Bourgeois, chargée de recherches, pour la traduction de l'article d'Ulrike Hansen, ainsi qu'Alissa Birle, stagiaire, Juliette Sondermeijer, ingénieur d'études, et Héloïse Cuillier, documentaliste, pour leur coopération aux travaux d'édition.

Solène Hazouard

Ingénieur d'études au CIRAC
René Lasserre

Directeur du CIRAC 



\section{Transition énergétique et Energiewende : vers une politique européenne de l'énergie?}

\section{Solène HAZOUARD, René LASSERRE}

Alors que débute la mise en œuvre des objectifs climatiques établis lors de la COP21 fin 2015 à Paris, il nous a semblé essentiel de valoriser les travaux entrepris ces deux dernières années dans le cadre d'un projet de formation-recherche à travers la parution d'un ouvrage collectif. Nous avons ainsi rassemblé dans un même volume les contributions de deux journées d'étude et d'un colloque final, réalisées par des chercheurs et des experts français, allemands et européens reconnus pour leur compétence et leur expérience sur les questions énergétiques et climatiques.

Dans un premier temps, cet ouvrage se propose de revenir sur les politiques énergétiques française et allemande de ces dernières décennies, et ce afin de mieux comprendre le contexte historique dans lequel s'inscrivent les stratégies de transition énergétique actuelles, lesquelles sont traitées dans une deuxième partie. II est ensuite question d'identifier des modèles de coopération franco-allemande à l'échelle locale, régionale et nationale, avant d'analyser plus en détail la transformation du marché de l'électricité et la nécessaire adaptation des réseaux électriques, pour garantir à la fois la compétitivité et la sécurité de l'approvisionnement. La cinquième partie de l'ouvrage offre l'occasion d'aborder la transition énergétique sous l'angle de la protection du climat et étudie l'impact de la COP21 pour l'Europe. Enfin, les derniers articles de l'ouvrage mettent en évidence le long chemin qu'il reste à parcourir avant de pouvoir réellement compter sur une politique européenne de l'énergie, les stratégies nationales étant encore aujourd'hui très différenciées, comme en témoignent les exemples du Royaume-Uni, de la Suède et de la Pologne.

Les politiques énergétiques en France et en Allemagne : éléments historiques

Face à la brusque augmentation des prix du pétrole dans les années 1970 dans un contexte de dépendance énergétique accrue, la France a choisi d'investir massivement dans le nucléaire et de diversifier ses approvisionnements pétroliers et gaziers. Dès lors, le secteur énergétique français se caractérise aujourd'hui par sa structure très capitalistique, par une faible empreinte carbone et par une électricité nucléaire à plus de $75 \%$. Il convient de noter également que depuis 1981, la part du pétrole et du charbon a reculé dans le mix énergétique (A. BELTRAN).

Après les chocs pétroliers, l'Allemagne a adopté pour sa part une stratégie alliant la revalorisation du charbon, la maitrise de la demande et le nucléaire civil (centralisé et privé), qui fournissait ainsi 40 \% de l'électricité en 1985. Dans le même temps s'est organisé un mouvement anti-nucléaire. Le premier tarif de rachat pour promouvoir les énergies renouvelables a été mis en place en 1991, 
ce qui a contribué à l'essor de l'éolien. Puis, le gouvernement rouge-vert sous Schröder a institutionnalisé le tournant énergétique entre 1998 et 2002, dans un contexte de libéralisation du marché européen de l'électricité qui a eu pour effet une concentration du secteur autour de quatre acteurs (E.ON, RWE, EnBW et Vattenfall) (S. C. AYKUT).

\section{La transition énergétique et l'Energiewende}

Cette deuxième partie s'ouvre sur une comparaison des projets français et allemand de transition énergétique. S'ils présentent des similarités quant à leurs objectifs, avec la réduction du volume des émissions de gaz à effet de serre (GES), le développement des énergies renouvelables, l'amélioration de l'efficacité énergétique et la réduction comparable, en chiffres absolus, de la production électronucléaire, de grandes divergences subsistent de part et d'autre du Rhin dans l'interprétation de la politique environnementale et le rôle de l'État (S. FISCHER).

Outre-Rhin, la loi de 2000 sur les énergies renouvelables (ErneuerbareEnergien-Gesetz, EEG) constitue un véritable tournant dans la politique énergétique. Après la catastrophe de Fukushima en 2011, Angela Merkel décide d'accélérer le processus de sortie du nucléaire tout en conservant les objectifs ambitieux de l'Energiekonzept de 2010, et notamment celui de porter à plus de $80 \%$ la part des énergies renouvelables dans la production d'électricité en 2050 (M. CRUCIANI). L'adoption de la transition énergétique, qui implique de nombreux défis sur le plan politique, économique, technologique et social, résulte par conséquent d'une longue culture de débat sur cette question (M. FISCHEDICK).

Dans les deux pays, l'un des grands enjeux réside dans le financement de la transition énergétique. En France, le secteur public ne pouvant pas supporter l'ensemble des coûts, le secteur privé doit rediriger ses investissements vers des projets «propres », et ce malgré le fait que les réglementations économiques et la politique industrielle continuent de favoriser la « croissance carbonée » et que le secteur financier n'intègre pas suffisamment les enjeux de la transition énergétique (R. MOREL/H. HAINAUT/I. COCHRAN). En Allemagne, la question du financement s'applique à deux domaines : l'efficacité énergétique d'un côté et, de l'autre, les énergies renouvelables et les systèmes énergétiques. Or le volume de financement des énergies renouvelables est beaucoup plus élevé que celui de l'efficacité énergétique, bien que ce dernier pilier soit tout aussi important dans la réussite de la transition énergétique (U. BENTERBUSCH).

\section{Exemples de coopération franco-allemande}

Si la France et l'Allemagne montrent des divergences concernant le recours au charbon ou au nucléaire et l'ampleur du développement des énergies renouvelables à ce jour, les deux pays se rejoignent dans la volonté de mettre 
en œuvre une transition énergétique, qui doit être portée par la technologie (J.-C. PERRAUDIN).

La coopération franco-allemande dans le domaine énergétique se joue à différents niveaux. À l'échelle locale par exemple, le projet TANDEM a vocation à établir, sur le long terme, des partenariats binationaux dans le domaine énergétique. Si les 11 partenariats communaux nés de ce projet contribuent, via un échange de connaissances et d'expériences, à la redynamisation des jumelages, ils sont néanmoins parfois confrontés à un manque de ressources et de soutien politique (A. TURFIN).

Sur le plan régional, on peut citer la collaboration trinationale francogermano-suisse dans la région du Rhin supérieur, qui repose sur l'implication de la société civile et des responsables politiques, économiques et scientifiques. En 2006, la Conférence franco-germano-suisse du Rhin supérieur a instauré une commission Climat et Énergie, qui a ensuite conçu le réseau, puis l'association TRION-climate. Cette plate-forme promeut le transfert de savoirs et d'expériences en matière d'efficacité énergétique et de développement des énergies renouvelables (J. OSER).

Enfin, l'Office franco-allemand pour la transition énergétique (OFATE) agit au niveau national. Fondé en 2006 à l'initiative des gouvernements français et allemand, il organise des conférences offrant la possibilité aux acteurs issus de l'industrie, des ministères, de la recherche et de la société civile de nouer des contacts pour travailler ensemble sur des solutions innovantes et propose à ses nombreuses PME adhérentes une veille régulière au niveau réglementaire, économique et technique. Il joue en outre le rôle d'intermédiaire pour les gouvernements et celui de consultant dans le cadre de la plate-forme énergétique franco-allemande de l'Agence française de l'environnement et de la maîtrise de l'énergie (ADEME) et de l'Agence allemande de l'énergie (Deutsche EnergieAgentur, dena) (S. RÖSNER).

\section{Défis et transformations du marché de l'électricité}

Dans le contexte de la transition énergétique, le secteur de l'électricité subit actuellement une profonde mutation. Chez le fournisseur allemand E.ON, on mise désormais sur les énergies renouvelables, les réseaux énergétiques et les solutions client (J. LANG).

$\mathrm{Au}$ sein de la Commission de régulation de l'énergie, on estime que le prix de l'électricité pour le consommateur final (particulier et professionnel) est susceptible d'augmenter en France en raison de la poursuite de l'ouverture des marchés de l'énergie, de la maintenance d'un parc nucléaire vieillissant, de la remise en concurrence des concessions hydrauliques souhaitée par la Commission européenne et de la poursuite du développement des énergies renouvelables (C. LEININGER).

En France, le recours accru à l'énergie éolienne et solaire nécessite de faire évoluer les infrastructures de réseau, en raison de la localisation différente des 
sources de production (H. MIGNON). Il s'agit de garantir la sécurité de l'approvisionnement en intégrant de plus en plus la notion de flexibilité, du fait de la variabilité de la production des énergies renouvelables. En Allemagne, le développement du réseau électrique est un enjeu d'autant plus crucial que le pays a choisi d'abandonner le nucléaire d'ici 2022 (U. HANSEN). Le renforcement des réseaux électriques nationaux et transnationaux constitue l'option de flexibilité la moins coûteuse. En matière de sécurité d'approvisionnement, la France est en faveur de l'introduction d'un mécanisme de capacité, tandis que l'Allemagne songe davantage à la mise en place d'une réserve stratégique opérée hors marché (D. PESCIA).

En raison du couplage des marchés en Europe, la sécurité de l'approvisionnement a cessé d'être une question nationale. Pour garantir l'approvisionnement en électricité, il convient d'intégrer complètement les énergies renouvelables dans le marché, de valoriser la flexibilité, de renforcer le signal prix du marché et d'évaluer les régulations européennes en vigueur avant d'envisager de nouvelles réglementations (W. VOGEL).

\section{La COP21 et la politique européenne de l'énergie}

La protection du climat - avec la réduction des émissions de $\mathrm{CO}_{2}$ comme objectif principal - est le moteur d'une politique énergétique durable. Elle est indispensable à une économie compétitive et moderne. En amont de la COP21, l'Allemagne a mis sur pied un programme d'action pour la protection du climat à l'horizon 2020 (Aktionsprogramm Klimaschutz 2020), qui contient plus de 100 mesures supplémentaires pour réduire les émissions de GES de $40 \%$ d'ici 2020 (B. GOEKE).

Au-delà des stratégiques adoptées au niveau étatique et des alliances internationales présentées lors de la COP21, les efforts à réaliser reposent avant tout sur la société civile et dépassent le cadre de la politique énergétique. En outre, le sommet de Paris n'est pas une fin en soi : des évaluations sont prévues tous les cinq ans à partir de 2018 et les contributions nationales seront réévaluées à la hausse tous les cinq ans à partir de 2020 (S. REICHE).

Il s'est produit un changement de paradigme entre la conférence de Cancún et celle de Paris. L'enjeu n'est plus de réduire les émissions de GES, mais d'atteindre la décarbonation des économies dans la seconde moitié de ce siècle, selon un principe d'universalité. Dans ce contexte, quatre questions se posent à l'Europe autour de la transition énergétique : celle de l'ambition, du projet industriel et économique, de la gouvernance et de la coopération (M. COLOMBIER).

L'Union européenne (UE) sera seule responsable de la mise en œuvre des moyens pour atteindre l'objectif, fixé lors de la COP 21, de réduction de ses émissions de GES de $40 \%$ d'ici à 2030 et de 80 à $95 \%$ d'ici à 2050 (par rapport à 1990). En matière d'énergies renouvelables, il existe des divergences 
croissantes entre les États membres quant aux objectifs à atteindre. La transition énergétique, qui engendre de l'incertitude sur le plan des investissements et dont le financement via des subventions n'est pas pérenne, gagnerait à être appréhendée à l'échelon européen, et non plus national (C. EGENHOFER).

Par ailleurs, l'objectif de la COP21 de limiter à $1,5-2{ }^{\circ} \mathrm{C}$ le réchauffement climatique d'ici à la fin du siècle (par rapport à la température de l'ère préindustrielle) correspond à la fourchette haute des ambitions de réduction des émissions de GES de l'UE et requiert une certaine flexibilité en termes de gouvernance, ainsi qu'une transformation du secteur énergétique. Pour réussir la transition énergétique dans l'UE, il ne suffit pas de renforcer le marché energy only et le système européen d'échange de quotas d'émission (SEQE). Il faut également procéder au retrait ciblé des anciennes centrales dépourvues de flexibilité et polluantes, assurer des revenus de marché stables pour les nouveaux investissements dans les énergies renouvelables et préserver l'adéquation du système (P. LITZ).

\section{Des stratégies énergétiques différenciées selon les pays}

Les énergies fossiles représentant actuellement $75 \%$ du mix énergétique de l'UE, les pays européens se rejoignent sur la volonté de décarboner l'économie, de garantir la sécurité de l'approvisionnement énergétique et d'assurer la compétitivité de leurs entreprises. Les trois derniers articles de l'ouvrage visent à identifier des convergences, des complémentarités, voire des oppositions dans les objectifs politiques du Royaume-Uni, de la Suède et de la Pologne (T. PELLERIN-CARLIN).

Au Royaume-Uni, les années 1990 ont été marquées par la libéralisation du secteur énergétique, le début du développement des énergies renouvelables et la « ruée vers le gaz », notamment pour réduire la dépendance au charbon. Lors de la décennie suivante, le pays s'est engagé à combattre le réchauffement climatique, de sorte que ces dernières années, il a nettement accru la part des énergies renouvelables dans sa production d'électricité et a entamé une sortie progressive du charbon, encouragé en ce sens par l'UE. Avant même le Brexit, qui génère de nombreuses incertitudes, la politique énergétique du Royaume-Uni différait de celle des États membres de l'UE sur des sujets comme le gaz de schiste, le nucléaire et le marché de capacité. Quelle que soit la relation qu'il entretiendra à l'avenir avec le marché unique, le pays reste lié à ses propres lois en matière de décarbonation (J. DUTTON).

En Suède, il est question de réduire à zéro les émissions nettes de GES d'ici à 2050, avec une priorité placée sur les transports. Le pays, qui voit ses émissions de $\mathrm{CO}_{2}$ reculer depuis 1970, prend part au SEQE et dispose d'une taxe sur le $\mathrm{CO}_{2}$ depuis 1991. À l'horizon 2020, il entreprend de diminuer de $40 \%$ ses émissions par rapport à 1990, de porter à au moins $50 \%$ la part des énergies renouvelables dans la consommation énergétique totale et de baisser 
de $20 \%$ l'intensité énergétique par rapport à 2008. Au printemps 2016, le gouvernement suédois a lancé une initiative en vue de devenir l'un des premiers pays à renoncer aux énergies fossiles dans le monde, ce qui implique une vaste coopération entre les organismes du secteur public et du secteur privé, la communauté scientifique ou encore les organisations non gouvernementales (T. B. JOHANSSON).

Si la politique énergétique de la Pologne à l'horizon 2030 et 2050 est encore en cours d'élaboration, le pays aspire néanmoins d'ores et déjà à la sécurité de l'approvisionnement, à la réduction des prix pour les particuliers, au développement des énergies renouvelables et à l'efficacité énergétique dans le contexte de la politique climatique de l'UE. Ainsi, dans son Plan de développement responsable, le ministère polonais du Développement économique ambitionne de «moderniser le secteur énergétique et de diversifier les sources d'énergie ». Cependant, la production d'électricité en Pologne repose actuellement à $85 \%$ sur le charbon, l'intensité énergétique estimée en parité de pouvoir d'achat (PPA) y est $15 \%$ plus élevée qu'en Allemagne, la pauvreté énergétique y demeure un vrai problème et le pays reste fortement dépendant du pétrole et du gaz russes (M. WA¿SIŃKI).

TOUTE POLITIQUe ÉNERGÉTIQUE doit concilier trois objectifs fondamentaux : la préservation du pouvoir d'achat des ménages et de la compétitivité des entreprises, le respect de l'environnement via la réduction des émissions de gaz à effet de serre et la sécurité de l'approvisionnement. Si les orientations en matière énergétique se décident sur le long terme, le contexte international a pour sa part évolué ces deux dernières années avec le Brexit, la COP21 et l'accroissement des tensions avec la Russie qui interroge sur l'approvisionnement énergétique, notamment pour la Pologne. Malgré une interdépendance croissante entre les systèmes énergétiques européens, les différences nationales persistent. Dès lors, la question est de savoir comment créer des convergences nationales rapidement et mettre en place une politique énergétique ambitieuse au niveau européen. 
$-1-$

LES POLITIQUES ÉNERGÉTIQUES en France et en AlLEMAGNe : ÉLÉMENTS HISTORIQUES 



\section{La France et sa politique de l'énergie (des années 1970 à aujourd'hui) ${ }^{1}$}

\section{Alain BELTRAN}

On peut parler de politique économique quand celle-ci s'inscrit sur le long terme, correspond à une priorité définie par les pouvoirs publics et est approuvée par la population car elle représente l'intérêt général et qu'en conséquence, des moyens importants lui sont consacrés. Ainsi a-t-on vu définir à la Libération des priorités industrielles destinées à reconstruire le pays, ainsi a-t-on vu à l'époque du Premier ministre puis président de la République Georges Pompidou (avec le soutien initial du général de Gaulle) des ambitions industrielles mises au premier plan de l'action publique pour assurer à la France son indépendance et son " rang ", terme essentiel des années 1960-1970. Parmi ces priorités industrielles, l'énergie a été dès l'origine un choix essentiel. Et malgré les alternances politiques, les renversements d'équilibre géopolitiques, les conditions économiques mouvantes, il n'est pas faux de dire que la politique énergétique française telle qu'elle a été définie une première fois dans les années 1970 tient plus de la constante que de la versatilité que l'on prête souvent à la France. Pour comprendre cette continuité finalement remarquable, un retour par l'histoire avec un regard ample et pluridisciplinaire s'avère essentiel. Surtout à l'heure où les changements et les relectures des politiques industrielles ayant un impact sur l'environnement deviennent des réalités mêlées d'urgence.

\section{Un pays dépendant sur le plan énergétique}

À l'inverse de la Grande-Bretagne riche de son charbon, des pays scandinaves ou de la Suisse riche de son hydroélectricité, la France n'a guère de ressources énergétiques à la dimension de son ambition industrielle. Sa principale richesse fut d'abord le charbon du Nord - Pas-de-Calais ainsi que de Lorraine, les autres sites (Massif central) n'ayant guère d'importance. Mais ce charbon était insuffisant en quantité, difficile à extraire, de qualité médiocre. Les Alpes et les Pyrénées fournissaient d'importants gisements hydrauliques mais plutôt excentrés et nécessitant la construction de grandes lignes de transport d'électricité. Point de pétrole, à part une découverte dans les Landes dans les années 1950 et une autre découverte de gaz naturel à partir de la fin des années 1950. Un fait peut résumer ce point faible de la France : ce pays fut le premier importateur mondial de charbon dans l'entre-deux-guerres.

Les pertes de devises et les dépendances ont marqué l'esprit des dirigeants au $\mathrm{XX}^{\mathrm{e}}$ siècle. Ce fut particulièrement le cas lors de la Première Guerre mondiale : d'une part, une grande partie des bassins houillers du Nord - Pas-deCalais étaient soit occupés par l'ennemi, soit sur la ligne de front donc inutilisables ; d'autre part, la France était totalement dépendante pour son pétrole de

\footnotetext{
${ }^{1}$ Article rédigé en juillet 2015, NdE.
} 
la Grande-Bretagne et surtout de la Standard Oil. Fin 1917, du fait du déclenchement de la guerre sous-marine, la France fut à deux doigts d'être privée d'approvisionnements en pétrole. Les imprévoyances d'avant 1914, la grande fragilité envers des énergies devenues stratégiques ont frappé les esprits des contemporains et ont décidé un certain nombre d'entre eux à réagir.

Les origines de la politique énergétique, de l'impératif énergétique pourraiton dire même, viennent donc de loin. C'est ainsi que la loi sur les forces hydrauliques en 1919 a donné à l'État la propriété sur les chutes d'eau. L'État créa en 1924 la Compagnie française des pétroles (CFP, ancêtre de Total) avec la part récupérée sur l'Allemagne de la Turkish Petroleum Company qui devint l'Irak Petroleum Company. Quatre ans plus tard, la loi sur les importations d'hydrocarbures (destinée à une longue vie) permettait aux pouvoirs publics de contrôler ce secteur et de construire rapidement une industrie nationale du raffinage, parmi les plus modernes d'Europe. Cet élan se poursuivit entre 1939 et 1944 avec la création de la Régie autonome des pétroles (RAP), qui trouva un peu de gaz, et de la Société nationale des pétroles d'Aquitaine (SNPA) en 1941, qui devait explorer une vaste zone dans le piémont pyrénéen, et l'instauration d'une loi sur le transport d'électricité, qui revenait à concentrer l'effort dans ce domaine. Et la liste pourrait être allongée par exemple avec la distribution d'électricité en zones rurales ou les recherches sur le « carburant national».

Tout cela ne formait pas à proprement parler une politique, mais en tout cas on pouvait y trouver les bases et l'affirmation d'une volonté.

\section{Un État dominant le secteur énergétique depuis la Libération}

Les événements qui ont eu pour cadre la Libération du pays et sa rénovation (essentiellement entre 1944 et 1946) doivent se comprendre à l'aune des énormes nécessités de la reconstruction d'un pays ruiné et aussi de la concentration des moyens entre les mains de l'État et de quelques personnalités. En fait, il n'était pas seulement question de reconstruire, il fallait aussi penser une nouvelle France à l'aune des expériences de l'entre-deux-guerres et des réflexions des Français libres. Du général de Gaulle à la majorité parlementaire tripartite (communistes, socialistes, républicains populaires ou MRP), la nécessité d'un État fort et de politiques volontaristes était une vue commune. D'où, dans le secteur de l'énergie, une vague de nationalisations qui toucha d'abord le secteur houiller (création de Charbonnages de France), l'électricité et le gaz (Électricité de France et Gaz de France, fondés en 1946), et enfin, la création de sociétés publiques dans le domaine pétrolier (le Bureau de recherche de pétrole puis l'Entreprise de recherches et d'activités pétrolières - ERAP - en 1966, ancêtres d'Elf-Aquitaine). Et pour l'avenir, la création du Commissariat à l'énergie atomique en 1945, car le général de Gaulle avait compris que les grandes nations devaient dominer le secteur nucléaire. Le tout était piloté par un ministère de la Production industrielle fort puissant car il détenait les matières premières essentielles en ces temps de forte pénurie. 
Tous ces moyens se conjuguèrent dans les années 1950 et 1960 (donc à la fois sous la $I^{\mathrm{e}}$ et la $\mathrm{V}^{\mathrm{e}}$ Républiques) pour aboutir à différentes facettes d'une politique qu'on peut qualifier d'indépendance nationale.

Ce fut d'abord la construction de barrages souvent impressionnants, baptisés «les cathédrales des temps modernes » par les contemporains. L'hydraulique a en effet l'avantage d'être national et renouvelable.

L'effort des mineurs («la bataille du charbon») est à noter aussi avec la modernisation des installations et de gros progrès de productivité, même si la fermeture des mines françaises fut décidée dans les années 1960, en particulier du fait d'un pétrole de plus en plus accessible.

En 1951, la découverte de gaz naturel dans la région de Lacq (près de Pau) a permis de transformer en profondeur cette industrie qui, de très marginale, devint un marché porteur.

Les années 1960 ont vu aussi la mise au point d'une filière nucléaire dite française - ou Uranium naturel graphite gaz (UNGG) -, qui d'ailleurs était tout aussi anglaise et canadienne que française. Toutefois, cette nouvelle preuve de l'indépendance technologique donna lieu à ce que les journalistes ont appelé « la guerre des filières ». En effet, si la technologie UNGG avait ses partisans, d'autres pensaient que les filières américaines comme le bouillant (boiling water reactor, BWR) de General Electric ou le pressurisé (pressurized water reactor, PWR) de Westinghouse étaient les seules techniques qui avaient de vrais débouchés internationaux. Il est intéressant de noter qu'en 1969, les Français abandonnèrent la filière UNGG pour prendre celle de l'américain Westinghouse : le fait de développer l'indépendance française ne signifiait pas la crispation sur les solutions seulement nationales. Toutefois, le fait d'avoir su mettre sur pied une industrie nucléaire nationale a donné les moyens et les compétences permettant de prendre des brevets américains et de faire aussi bien sinon mieux que l'industriel d'origine.

Enfin, après des recherches en métropole et dans les différentes parties de l'ex-Empire, la France en 1956 découvrait du gaz et du pétrole dans le désert saharien. En quelques années, ce pétrole pouvait être dirigé vers la France, ce qui changeait complètement la donne : désormais, la France avait son indépendance énergétique, même si la fin de la guerre d'Algérie (1962) signifiait que cette manne pouvait être éphémère.

En conséquence, les sociétés créées après la guerre, comme la Société nationale des pétroles d'Aquitaine ou la Régie autonome des pétroles, sont devenues de vraies et grandes compagnies, sans oublier la Compagnie française des pétroles, qui elle aussi fit des découvertes dans le désert saharien. Le secteur public pétrolier se concentra une première fois en 1966 autour de l'ERAP, qui lança l'année suivante sa marque d'essence, ELF. La France se trouvait donc avec deux pôles pétroliers : un public, l'autre quasi privé (CFP).

Ce secteur d'État consacré à l'énergie avait une unité plus profonde qu'on ne pouvait le penser si on s'arrête un instant sur la sociologie de ses dirigeants 
et les motivations qui les animaient. En effet, souvent issus de la prestigieuse École polytechnique, et en particulier des grands corps de cette école - cette expression désigne les élèves sortis premiers du classement final qui choisissent comme écoles d'application les Mines (le corps le plus prestigieux) ou les Ponts et Chaussées -, les dirigeants des grandes sociétés françaises nationales partageaient certaines valeurs communes. Le service public, l'intérêt général, le rang de la France parmi les grandes puissances ainsi qu'une foi dans le progrès par la science et la technique : telle était la base commune aux X-Mines et X-Ponts (les deux corps principaux de Polytechnique).

De plus, les grandes directions des ministères, et en particulier celui de l'Industrie, étaient peuplées de responsables techniques en général issus des grandes écoles et partageant les mêmes idéaux.

Dernière base de ce «triangle de stabilité »: les organisations syndicales, dominées par la Confédération générale du travail (CGT), soutenaient les efforts d'indépendance nationale et croyaient aussi que la recherche, la science et la technique pourraient apporter le progrès. Certes, il existait des failles dans ce système, comme le deuxième syndicat français né en 1964 - la Confédération française démocratique du travail (CFDT) -, nettement plus critique envers le progrès technique. Mais globalement, la politique énergétique répondait aux grandes attentes de la société française.

\section{La réaffirmation d'une politique d'indépendance lors du premier choc pétrolier}

Pourtant, si la politique d'indépendance justifiait des programmes de long terme, comme le nucléaire dont on pensait qu'il atteindrait son apogée dans les années 1980-1990, d'autres phénomènes venaient contrarier cette orientation.

La baisse continue du prix du pétrole au cours des années 1960, le développement de l'automobile et donc de la consommation d'essence, le déclin inexorable du charbon national, qui était avant tout un problème d'ordre social, tout cela entraînait une dégradation de la balance énergétique. La France devenait largement importatrice de pétrole et de gaz naturel, de charbon aussi. Au début des années 1970, les trois quarts des besoins nationaux en énergie étaient importés. Et les prévisions de consommation restaient importantes, basées sur le pétrole, liant étroitement expansion économique et besoins en énergie. Malgré la politique menée depuis des années vers une plus grande indépendance, les faits étaient contradictoires et montraient que la France - comme d'autres pays européens - devenait dépendante des pays exportateurs de pétrole.

Le premier choc pétrolier d'octobre 1973 et des mois suivants ramena brutalement la France à la réalité, mais également à son attitude développée depuis des années. Certains ont jugé la réactivité française comme une rupture : nous pensons en fait qu'elle fut un retour, une mobilisation pour retrouver les fondamentaux de sa politique énergétique développée depuis la Libération. La réaction fut donc centralisée, massive et rationnelle. Si la partie la plus connue est le 
lancement d'un grand programme électronucléaire en mars 1974 (dit Plan Messmer, du nom du dernier Premier ministre de Georges Pompidou), on ne peut oublier d'autres aspects: le refus d'intégrer l'Agence internationale de l'énergie, jugée trop dominée par les États-Unis (ce qui va bien dans le sens de l'indépendance vis-à-vis des « Grands » qui est un symbole de la politique gaullienne et post-gaullienne), la diversification des approvisionnements pétroliers et gaziers ainsi qu'un vaste et médiatisé programme d'économies d'énergie. Si l'on parla beaucoup du solaire dans les années 1970 (construction de la centrale Thémis dans les Pyrénées françaises), les efforts dans le domaine des énergies dites «nouvelles » (on dirait renouvelables aujourd'hui) furent en fait limités par l'option nucléaire qui était prioritaire. Le programme français se développa sur les connaissances et expériences antérieures, avec l'appui majeur d'une entreprise publique (Électricité de France, EDF) et le soutien sans faille des différents présidents de la République et du syndicat CGT.

Cependant, les années 1970 ont vu se développer les premières contestations anti-nucléaires qui se sont focalisées sur le chantier du surgénérateur Superphénix (près de Lyon) et sur un projet de centrale en Bretagne (Plogoff). En 1981, lors de l'alternance politique qui vit arriver la gauche socialiste au pouvoir, le projet breton fut arrêté mais le reste du programme alla à son terme.

Dès lors, et jusqu'à une période récente, les grands traits de la politique énergétique française se sont affirmés. Le premier objectif à atteindre était de diminuer la dépendance et les importations : de $75 \%$ du total des besoins nationaux, les achats en produits énergétiques passèrent à $50 \%$, niveau stable jusqu'à aujourd'hui. Le pétrole et le gaz sont fatalement importés, mais l'électricité nucléaire (près de $75 \%$ de la production française) assure l'essentiel de la demande et même peut être exportée chez nos voisins. La consommation de pétrole a nettement diminué dans les années 1970 et s'est stabilisée par la suite. La diversification a été obtenue en diminuant les achats au Proche et au Moyen-Orient, en augmentant les importations venant de l'URSS et de l'exURSS, d'Afrique et surtout de mer du Nord, où les pétroliers français comme Elf-Aquitaine (société nationale créée en 1976 par la fusion de l'ensemble du secteur pétrolier public) et CFP-Total ont joué un rôle important, souvent en collaboration.

Le secteur nucléaire français est dominé par des entreprises publiques comme EDF, le Commissariat à l'énergie atomique pour la partie recherche et une société de construction (Framatome, puis AREVA). Cet édifice est cependant très sensible aux événements extérieurs ; en effet, l'une des particularités du secteur nucléaire est d'être en résonance avec les contestations ou accidents dans le monde. En ce sens, la catastrophe de Tchernobyl a sans doute pour la première fois fait vaciller l'opinion publique française, même si les sondages ne sont pas simples à interpréter. Le message sur la sûreté du nucléaire a été ébréché, mais globalement la France a été moins sensible par exemple au «nuage » de Tchernobyl que nos voisins allemands. Le changement dans le secteur élec- 
trique n'en a pas moins été sensible, puisque la croissance de la consommation d'énergie électrique était de 6-7\% depuis les années 1920, soit un doublement tous les dix ans. À partir du premier choc pétrolier, ce chiffre a diminué et sur les dernières années, il a même tendu vers zéro ou même moins, ce qui est une rupture essentielle.

La courbe de la consommation de gaz naturel est quant à elle très différente : elle marque une progression constante jusque vers 2005 puis connait un palier. Cette énergie, on l'a vu, a connu son essor à partir de la découverte du gisement de Lacq. Par la suite, la demande étant suffisante, il fallut passer des contrats avec les Pays-Bas, l'Algérie, l'URSS, la Norvège... L'avantage était une certaine répartition, mais avec la fin du gisement de Lacq, la France devenait totalement dépendante d'approvisionnements extérieurs pour son gaz naturel, Gaz de France (GDF) étant en définitive distributeur et importateur.

Quant au charbon, les dernières mines ont fermé progressivement (l'ultime en Lorraine) et les pouvoirs publics ont essayé de redynamiser un tissu industriel touché par le déclin des activités issues de la première révolution industrielle (charbon, acier, textile).

\section{Une adaptation tardive aux nouvelles orientations européennes en matière d'énergie}

La réponse aux chocs pétroliers en France fut donc l'affirmation d'une politique d'indépendance nationale qui s'appuyait sur des intérêts hexagonaux avec des moyens largement définis par l'État. Certains ont pu y voir l'affirmation d'une politique de «prestige », mais en fait le système français était bien adapté aux grands programmes industriels (voir par exemple le téléphone, le train à grande vitesse, l'aéronautique et le spatial).

Dans cet environnement, la Communauté économique européenne (ou pour le nucléaire EURATOM) ne jouaient pas un rôle majeur. Toutefois, à partir des années 1980, même si ce n'était pas la politique énergétique française qui était visée en priorité, la structure de cette politique a correspondu de moins en moins aux directives européennes. En effet, Bruxelles prônait la concurrence, la liberté des marchés, la fluidité et la transparence. Or Paris défendait des entreprises publiques, plus ou moins assises sur un quasi-monopole, avec un marché très fermé. On constata bien durant des années que les différents gouvernements français, quelle que soit leur couleur, ont défendu ce qu'on pourrait appeler le « modèle français » d'une certaine politique énergétique. Et on pourrait dire que la puissance en particulier d'EDF, en définitive premier électricien au monde, assis sur un parc de 58 réacteurs nucléaires pour 19 centrales, surprenait, agaçait même.

Les années 2000 ont profondément changé la donne dans tous les secteurs, et pas seulement du fait des directives européennes. Le secteur pétrolier français avait la caractéristique inusitée d'avoir deux «champions nationaux », la socié- 
té nationale Elf-Aquitaine, privatisée en partie en 1996, et la société Total. En général, un pays de taille moyenne ne possédait qu'une entreprise pétrolière, mais le cas français s'expliquait par une évolution historique originale. C'est en fait la société privée Total qui en 1999-2000 sut tirer avantage de la situation en absorbant d'abord le belge Fina, puis l'année suivante Elf-Aquitaine. Par voie de conséquence, cette concentration donna naissance à un grand du pétrole mondial (dans les cinq premiers), ce qui pour un pays sans hydrocarbures et latecomer peut paraître comme une réussite.

Dans le domaine gazier, pour répondre à une offensive italienne, l'État décida assez brusquement de former un groupe industriel entre la société nationale Gaz de France et la Compagnie de Suez, vénérable société qui avait été à la base de la construction du canal du même nom (nationalisé en 1956), mais avait aussi d'importants intérêts dans l'eau, l'hygiène et l'énergie (centrales nucléaires belges, installations hydroélectriques). Ce mariage entre utilities restait marqué par la domination du secteur gazier qui semblait avoir un grand avenir. De plus, directement en lien avec la transposition des directives bruxelloises, les sociétés nationales du secteur public virent leur statut modifié de façon sensible. EDF et GDF furent transformées en sociétés anonymes, leur capital fut ouvert et ces entreprises qui étaient intégrées (c'est-à-dire qui réunissaient les fonctions de production, transport et distribution) furent séparées, d'abord comptablement puis dans leurs fonctions. Ainsi GDF-Suez était une société productrice en concurrence avec d'autres fournisseurs ; mais le transport de gaz (Gestionnaire de réseau de transport de gaz, GRTgaz) et la distribution (Gaz réseau distribution France, GrDF) devaient être neutres, transparents et objectifs par rapport à l'ensemble des fournisseurs.

Il en allait de même pour EDF, relevant du secteur concurrentiel, tandis que le transporteur RTE (Réseau de transport d'électricité) et le distributeur ERDF (Électricité réseau distribution France) se devaient de n'avantager aucun fournisseur, y compris leur fournisseur historique (c'est-à-dire EDF). Les choses n'étaient pas simples car, en même temps, EDF était un groupe avec deux filiales à 100 \% (RTE et ERDF) qui se devaient d'afficher leur totale indépendance par rapport à leur maison mère.

Au passage, le principe de spécialité qui faisait que les gaziers ne vendaient que du gaz et les électriciens que de l'électricité fut aboli. Les marchés intérieurs furent progressivement ouverts à la concurrence : d'abord les usagers industriels, puis les usagers commerciaux et enfin les consommateurs domestiques. Il était donc possible de changer de fournisseur. En réalité, quelques années après cette mutation importante, les consommateurs sont restés en majorité fidèles à leur fournisseur historique et à ses tarifs régulés. L'ouverture à la concurrence reste un peu plus visible dans le domaine gazier que dans le domaine électrique. Les pouvoirs publics ont essayé de favoriser une certaine fluidité du système en essayant de mieux répartir le «nucléaire historique », sans résultats très probants pour le moment. Tout ce système était surveillé par une entité 
nouvelle, indépendante, la Commission de régulation de l'énergie (CRE). D'un point de vue législatif, si la loi française avait permis tous ces changements, d'un autre côté, en 2000, les textes officiels concernant le gaz et l'électricité réaffirmaient certaines obligations de service public : l'obligation d'assurer la sécurité d'approvisionnement par exemple. Là aussi, une certaine continuité reste visible par-delà les changements structurels.

Enfin, les directives bruxelloises sur différents points ont été plus rapidement transcrites en droit français, qu'il s'agisse des objectifs environnementaux, des normes $\mathrm{CO}_{2}$ ou du développement de nouveaux réseaux pour assurer une meilleure fluidité.

\section{Bilan de quarante années (et plus...) de politique énergétique}

En termes chiffrés, les résultats de la politique énergétique française peuvent être résumés. La production d'énergie primaire est passée entre 1973 et 2012 de 44 millions de tonnes équivalent pétrole (tep) à 136 millions de tep, essentiellement du fait de la croissance remarquable de la production d'énergie nucléaire (l'électricité française est nucléaire pour les trois quarts). Le taux d'indépendance énergétique, qui était tombé à $23,9 \%$ en 1973 , est remonté à $49,5 \%$ en 1990 et a peu évolué depuis lors (51,2 \% en 2012) car les importations d'hydrocarbures pèsent toujours sur notre économie. Mais le déficit de la balance énergétique, qui atteint 68,7 milliards d'euros en 2012, reste un vrai fardeau puisqu'il est à lui seul équivalent au déficit commercial (67,2 milliards d'euros). Ce déficit, et ce n'est pas une surprise, est dû pour trois quarts aux produits pétroliers et pour environ un quart aux importations de gaz naturel.

À l'inverse, la consommation d'énergie primaire est désormais maîtrisée et ne progresse plus depuis une bonne dizaine d'années ; on remarque même un léger reflux. Avec un indice un peu plus fin, la consommation d'énergie par unité de produit intérieur brut (PIB), les résultats sont nettement plus illustratifs puisqu'entre 1970 et aujourd'hui, la baisse a été pratiquement de $50 \%$. Il est clair désormais que la croissance et la consommation d'énergie ne sont plus corrélées.

La part des énergies renouvelables (essentiellement de l'hydraulique en France, même si le solaire et l'éolien apparaissent désormais dans les bilans) reste modeste par rapport aux deux grandes masses que représentent nucléaire et pétrole.

Si la part du pétrole a baissé dans les bilans, il est à noter que sur quarante ans, celle du gaz naturel a progressé. D'une part, cette énergie est la moins polluante des énergies fossiles, d'autre part, elle est bien diversifiée du point de vue des producteurs. Certains prédisent un âge d'or au gaz naturel car il serait par excellence l'énergie de transition pour pallier l'intermittence des énergies renouvelables.

Si l'on regarde les résultats par secteurs de consommation, on constate que l'agriculture ne pèse guère sur les bilans, que le secteur industriel a réalisé de très sensibles économies, mais qu'à l'inverse, transports et résidentiel restent de 
gros consommateurs. C'est dans ces directions que l'effort devra être fourni, mais sans doute avec une forte résistance du système.

Il reste enfin un indice qui a pris toute sa valeur aujourd'hui : celui des rejets de carbone. Après une progression régulière, un palier a été atteint dans les années 1970. Puis, résultat conjugué du développement du nucléaire (qui ne rejette pas de carbone) et des efforts réalisés dans de nombreux domaines d'activité, le niveau des rejets a baissé régulièrement. Cette diminution entre 1973 et 2013 a atteint 30 \% (environ 10 \% depuis le début des années 1990, car les nations choisissent différents points initiaux lors des conférences internationales). La France met en avant cette basse production de carbone, même si des progrès sont encore largement possibles.

LA POlitique ÉnergétiQue de LA FrAnCE semble s'inscrire aujourd'hui dans un contexte nouveau du fait des restructurations demandées par l'Union européenne. Pourtant, à y regarder de plus près, une bonne partie de l'esprit de la construction des années 1960-1980 reste présente. Le nucléaire domine toujours la production d'électricité, même si les projets actuels tendent à diminuer sa part relative. Les énergies renouvelables sont appelées à se développer, mais restent modestes par rapport à un certain nombre de nos voisins. La France continue de diversifier ses approvisionnements pour ne pas être trop dépendante d'un fournisseur, comme dans le cas de la Russie au moment de la crise ukrainienne (seulement $15 \%$ du gaz naturel consommé en France vient de Russie).

Toutefois, le clivage entre la gauche et la droite reste assez visible dans le domaine du nucléaire car la droite française y est favorable pour des raisons d'indépendance nationale, la gauche souhaitant plus d'énergies renouvelables, en particulier du fait du poids de l'électorat vert. Mais, globalement, l'arrivée d'une nouvelle majorité ne se concrétise pas par une révision déchirante de la politique énergétique française. Et cela pour plusieurs raisons, la principale étant que ce secteur étant très capitalistique, les changements ne peuvent être que très progressifs. Ainsi, il est clair que les politiques d'économies d'énergie ont commencé par les objectifs les plus aisés à remplir. Mais quand il s'agit du secteur des transports ou de l'isolation des maisons individuelles, les résultats ne peuvent se faire sentir que par une politique incitative forte (le gouvernement actuel ne voulant pas d'une politique «punitive ») et sur la longue durée.

En bref, passer d'un modèle centralisé et fortement étatique à un modèle décentralisé ouvert aux initiatives citoyennes n'est pas chose simple dans un pays comme la France. Toujours est-il que les exemples étrangers (en particulier l'abandon du nucléaire par l'Allemagne) ont montré que d'autres voies sont possibles. Certains pensent que les possibilités techniques nouvelles apportées par l'informatique permettent d'imaginer des systèmes décentralisés qui d'ailleurs apparaissent ici ou là. La politique énergétique est revenue au premier plan du débat politique, ce qui n'est d'ailleurs pas toujours un gage de sérénité pour des décisions si stratégiques. 



\title{
De la reconstruction à l'Energiewende. Continuité et changement dans les politiques énergétiques allemandes ${ }^{1}$
}

\author{
Stefan C. AYKUT
}

La France et l'Allemagne, pays voisins dont l'histoire est intimement liée, anciennes ennemies jurées formant aujourd'hui le " couple franco-allemand 》, sont entrées de plainpied dans la modernité technologique en réformant à une vitesse impressionnante leurs économies dévastées après la Seconde Guerre mondiale. Ce changement profond s'est fait plus ou moins simultanément, dans des périodes de croissance soutenue que les deux sociétés commémorent et idéalisent aujourd'hui respectivement comme les "Trente Glorieuses » et le Wirtschaftswunder, le " miracle économique ».

Dans les deux pays, les politiques énergétiques, et en particulier celles en faveur de l'électrification, ont joué un rôle central dans ce projet de modernisation. Si les orientations globales de ces politiques ont pu converger par le passé, les différences sont désormais frappantes : tandis qu'en France, la densité des centrales nucléaires et leur part dans la production d'électricité sont les plus hautes au monde, l'Allemagne voisine a arrêté la construction de nouveaux réacteurs au début des années 1980 et la part du nucléaire dans la production d'électricité n'a jamais dépassé $40 \%$. Le paysage rural et urbain de l'Allemagne des années 2010 se distingue d'ailleurs par ses centaines de milliers de panneaux solaires sur les toits et par ses parcs d'éoliennes dans les campagnes, ce qui témoigne d'une révolution énergétique - l'Energiewende ou "tournant énergétique » - en marche. Comment expliquer ce décalage? Cette contribution a pour objectif de livrer les clefs historiques pour comprendre l'évolution récente des politiques énergétiques allemandes. Pour cela, nous nous concentrons sur le secteur de l'électricité et sur la période qui a précédé le tournant énergétique allemand des années 2000.

\section{La structuration du secteur de l'électricité}

Historiquement, le secteur énergétique allemand s'est organisé autour de monopoles régionaux. Il procède d'une gestion complexe qui implique les échelles communale et régionale mais qui n'accorde finalement qu'un rôle limité à l'État fédéral. Cette organisation a ses origines au début du $\mathrm{XX}^{\mathrm{e}}$ siècle. Elle est le fruit d'une concurrence entre l'État central et les autres niveaux de gouvernement pour la régulation du domaine et d'une opposition entre les tenants d'une organisation publique (des entreprises détenues par l'État) et les partisans d'une organisation privée du secteur.

En 1917, vers la fin de la Première Guerre mondiale, le gouvernement du Reich fut pour la première fois autorisé à formuler un cadre législatif pour toute

\footnotetext{
${ }^{1}$ Article rédigé en juin 2015, NdE.
} 
l'Allemagne. L'intérêt de l'État fédéral dans le secteur était double. D'une part, il s'agissait de faire avancer le vaste chantier d'extension et d'homogénéisation des réseaux électriques. D'autre part, les revenus de ce secteur stratégique en plein essor aiguisaient l'appétit financier de l'État. Différentes tentatives de régulation et de centralisation dans le domaine échouèrent toutefois dans la période de l'entre-deux-guerres, principalement à cause de l'opposition des communes et des Länder, les États fédérés.

Il faut revenir sur le statut particulier des échelles locale et régionale pour comprendre les événements de l'époque mais aussi la situation actuelle. Deux concepts juridiques allemands leur confèrent un rôle central dans le domaine énergétique :

- la Daseinsvorsorge (service public), qui signifie que les communes ont le devoir de garantir les services d'intérêt général au niveau local, et notamment la fourniture d'électricité. Cela a abouti à la création de régies communales, certaines villes faisant aussi appel à des acteurs privés pour garantir ce service public ;

- le Wegemonopol («monopole des routes»), en vertu duquel communes et Länder possèdent les routes nécessaires à la construction de réseaux et à l'acheminement de l'énergie et accordent des concessions aux entreprises qui leur versent en contrepartie une redevance ${ }^{2}$.

Grâce à la pratique des concessions, les communes participent au profit des fournisseurs d'électricité (il en est de même pour le gaz et l'eau) et les fournisseurs gagnent l'accès exclusif à un territoire : c'est l'origine des monopoles régionaux (Krisp, 2007, p. 16). À partir du début du XX $X^{\mathrm{e}}$ siècle, les communes se sont investies aussi activement dans l'électrification, en (r)achetant des réseaux, en construisant des centrales et en entrant dans le capital des entreprises. Autre acteur important, les États fédérés se sont nettement impliqués dans la première phase d'électrification, notamment dans la construction des réseaux et des interconnexions sur leur territoire. Quand le gouvernement fédéral décidait de s'engager dans le secteur, ils craignaient de se voir dépossédés des fruits de leurs efforts et cherchaient à limiter l'emprise du Reich par une politique de création d'entreprises régionales de distribution d'électricité (Stier, 1999, p. 413 ; Krisp, 2007, p. 18).

\section{Des monopoles régionaux publics et privés}

À la fin de la République de Weimar, ces tensions débouchèrent sur une guerre ouverte opposant les grands monopolistes détenus par l'État (Bavière, Prusse, Hambourg, etc.) et les entreprises privées auxquelles participaient, en suivant le modèle du géant RWE, la plus grande entreprise du secteur, les communes et l'industrie lourde. Chacun essayait alors d'agrandir son territoire et ses parts de

\footnotetext{
${ }^{2}$ Pour une analyse de cette construction particulière du droit allemand, voir Pfaffenberger et al. (1992, p. 19).
} 
marché aux dépens des autres. Le secteur fut finalement stabilisé en 1927, quand la «paix de l'électricité » entre la Preussische Elektrizitäts-AG et RWE fixa des lignes de démarcation définitives.

Quelques années plus tard, en 1935, le gouvernement national-socialiste donna un premier cadre national aux politiques énergétiques allemandes, avec la loi sur la promotion de l'industrie de l'énergie ${ }^{3}$. Cette loi a formé, malgré quelques modifications à la marge, la base de la régulation du secteur jusqu'en 1998. Visant avant tout à garantir la sécurité d'approvisionnement en vue de préparer l'effort de guerre, elle a codifié la pratique courante des contrats de concession et des contrats de démarcation. En vertu du caractère de « monopole naturel» des réseaux, elle a exclu toute concurrence dans le secteur de l'énergie. En contrepartie de la pérennisation du système monopoliste et décentralisé, la loi introduit plusieurs principes :

- les entreprises du secteur doivent fournir de l'électricité toujours et partout de façon pérenne (Kontrahierungszwang) ;

- des instances de surveillance contrôlent la distribution d'énergie et la construction de centrales ;

- les administrations des États fédérés contrôlent les prix pour les consommateurs finaux (ménages et petites entreprises), alors que les grands clients industriels peuvent négocier leurs contrats d'approvisionnement directement avec les énergéticiens.

La loi a pérennisé aussi le «pentagramme de fer» (Kleinwächter, 2007) de la régulation du secteur dont les acteurs sont l'État fédéral, les Länder, les communes, les entreprises de production et de distribution d'électricité et les grands clients industriels.

Une caractéristique de ce système réside dans l'imbrication et l'interdépendance des différents acteurs. Ainsi, même si l'industrie énergétique n'est pas nationalisée, l'État joue un rôle central dans le domaine, agissant à travers ses différents organes à la fois comme acteur du marché (les entreprises publiques), comme régulateur et comme instance de contrôle. Quant aux électriciens et aux grands clients industriels, ils sont consultés lors des projets législatifs les concernant, et il n'est pas rare que des politiciens ou hauts fonctionnaires soient employés par des entreprises énergétiques au terme de leur carrière politique (Gammelin/Hamann, 2005).

De manière plus générale, le secteur énergétique forme le cœur du «corporatisme à l'allemande », caractérisé par un réseau dense de continuités personnelles dans les conseils de surveillance et les directions d'entreprises et par des participations mutuelles entre entreprises et banques. Les États fédérés, qui tiennent une participation dans l'entreprise énergétique locale, sont d'ailleurs représentés dans les conseils de surveillance de «leur» énergéticien (Beyer, 2002, p. 1).

\footnotetext{
${ }^{3}$ Gesetz zur Förderung der Energiewirtschaft, EnWG (Bier, 2002).
} 


\section{Un système énergétique bâti sur le charbon}

En termes de sources énergétiques, l'Allemagne a traditionnellement favorisé le charbon $^{4}$, une ressource abondante sur son territoire, qui a joué un rôle décisif dans l'industrialisation et l'électrification du pays.

Pour les Allemands de l'Ouest, la reprise progressive de l'extraction charbonnière, après une sévère période de pénurie de charbon dans l'immédiat après-guerre ${ }^{5}$, signifiait non seulement le retour du chauffage pour des millions de ménages mais elle était aussi devenue le symbole de la reconstruction, en assurant du travail pour des centaines de milliers de mineurs et les métiers associés et en créant les conditions nécessaires pour l'essor industriel du pays. En 1950 , le charbon représentait près de $90 \%$ de l'énergie consommée en Allemagne et 103 millions de tonnes de houille étaient extraits dans les charbonnages allemands. Ce secteur employait plus de 433000 personnes (Huske, 2006), chiffre auquel il faut ajouter quelques dizaines de milliers d'emplois dans l'extraction de lignite. Ces chiffres aident à comprendre l'importance économique mais aussi symbolique de cette ressource dans l'histoire allemande récente (Evrard, 2014). Cette importance symbolique est soutenue par une alliance hétéroclite mais puissante entre les syndicats, les énergéticiens, les grands clients industriels (surtout la sidérurgie), les ministres-présidents des États fédérés charbonniers et le parti social-démocrate (SPD), traditionnellement proche du milieu des mineurs.

En résumé, on peut dire que les caractéristiques du système énergétique allemand étaient stabilisées dès la fin des années 1920 et qu'elles ont été codifiées au milieu des années 1930 (Stier, 1999, p. 413). Ce système a été la source d'une stabilité d'approvisionnement remarquable tout en ne demandant qu'un effort relativement limité à l'État, puisque l'investissement dans les réseaux et les infrastructures pouvait se faire grâce à la rente de monopole garantie aux entreprises électriques. En revanche, la complexité des structures du secteur rendait peu transparents les mécanismes de formation des prix et compliquait les tentatives de réforme du secteur.

\section{La naissance d'une politique énergétique fédérale}

Le secteur connut deux chocs successifs dans les décennies d'après-guerre : la « crise du charbon » des années 1950-1960 et les crises pétrolières des années

\footnotetext{
${ }^{4}$ On distingue deux formes de charbon, la houille, dont le contenu énergétique est supérieur et qui est subventionnée en Allemagne, et le lignite, dont l'extraction est moins onéreuse mais très polluante parce qu'elle se fait à ciel ouvert. L'Allemagne dispose de grandes réserves de houille, mais elle est surtout le premier pays en termes de production et le deuxième en termes de ressources de lignite. Par un souci de facilité de lecture, nous parlerons de « charbon » pour désigner les deux.

${ }^{5}$ De 130 millions de tonnes de houille extraite en 1939, la production tombe à 33 millions de tonnes. On parle alors de Kohlenot, «pénurie de charbon ».
} 
1970, ce qui a fait apparaître une implication plus directe de l'État fédéral comme étant une nécessité.

Le premier de ces événements, la crise du charbon, avait ses origines dans la mondialisation du marché des ressources énergétiques au cours de laquelle du pétrole et du charbon peu coûteux inondaient le marché allemand. La concurrence internationale exposait au grand jour les déficiences du secteur charbonnier, fortement subventionné jusque dans les années 1950 par peur d'un manque de capacités d'extraction dans un contexte de forte croissance de la demande, soit un doublement par décennie depuis la fin de la guerre. À cause d'un coût d'extraction comparativement élevé ${ }^{6}$, un secteur industriel entier entrait alors en déclin : si le charbon fournissait encore $88 \%$ de l'énergie primaire en 1950, sa part chuta à $74 \%$ en 1960 , puis à $28 \%$ en 1970 . Le pétrole, qui ne contribuait qu'à hauteur de $4 \%$ à la production énergétique en 1950 , passa à $53 \%$ en $19700^{7}$. Trois ans plus tard, la production de houille allemande n'atteignait plus que 80 millions de tonnes, entraînant des fermetures de charbonnages et une chute du nombre d'employés travaillant dans le secteur ${ }^{8}$.

Ces évolutions ont donné lieu à de vifs débats au sein du gouvernement de l'époque, opposant le chancelier, Konrad Adenauer, et son ministre de l'Économie, Ludwig Erhard. Si le premier plaidait pour la protection du secteur, le second, père du Wirtschaftswunder et fervent partisan du libéralisme économique, défendait une ligne politique qui minimisait l'intervention de l'État. Ces tensions furent exacerbées par le fait qu'en 1955, l'Allemagne était pour la première fois devenue importatrice nette de ressources énergétiques.

C'est dans ce contexte que le Parlement allemand créa en 1959 une commission d'enquête sur l'énergie, en lui donnant comme mission d'établir une prévision énergétique qui établirait différents scénarios d'évolution du secteur charbonnier allemand ${ }^{9}$. Les résultats de l'étude étaient alarmants, encourageant l'adoption d'une série de mesures par des gouvernements successifs, dont différentes lois régulant la production d'électricité et l'importation de charbon ${ }^{10}$, qui visaient à protéger le marché allemand et à ralentir le déclin des charbonnages. Plus généralement, la politique énergétique fédérale naissante s'inscrivait dans une nouvelle approche des politiques économiques. Résumée sous la formule de Globalsteuerung, le «pilotage global », et codifiée en 1967 dans la loi sur la stabilité économique (Stabilitätsgesetz), cette doctrine fixe comme objectifs des politiques économiques la stabilité des prix, une balance commerciale équili-

\footnotetext{
${ }^{6}$ Pour des raisons géologiques et à cause du différentiel des salaires entre les pays, le charbon allemand coûte alors jusqu'à 15 marks de plus par tonne que celui importé des États-Unis, du Canada ou de Chine.

${ }^{7}$ Chiffres de la Arbeitsgemeinschaft Energiebilanzen (http://www.ag-energiebilanzen.de/, consulté le 24/05/2014).

${ }^{8}$ En 1995, le secteur n'emploie plus que 141000 personnes.

${ }^{9}$ Voir Aykut (2015) pour une analyse de cette commission appelée Energie-Enquete.

${ }^{10} \mathrm{La}$ première de cette série est la loi pour la promotion de l'utilisation du charbon dans les centrales (VerstromG 1) du 12 août 1965. L'importation de charbon est d'ailleurs limitée par des quotas d'importation (Kohlezollkontingentgesetz). De nouvelles lois ou modifications des lois existantes en 1966, 1974, 1976, 1977 et 1980 montrent que l'importance accordée à la question ne s'amenuise pas (Krisp, 2007, p. 26-27).
} 
brée, une croissance économique forte et un niveau d'emploi élevé. Étant donné que les contradictions entre ces objectifs rendent leur poursuite simultanée quasiment impossible, on parle aussi du «carré magique » des politiques économiques (Meyer-Abich/Dickler, 1982, p. 223).

De telles contradictions étaient particulièrement visibles dans les politiques énergétiques : si l'accès à des ressources énergétiques bon marché était considéré comme indispensable pour satisfaire au moindre coût la demande énergétique, le développement de ces énergies - principalement du charbon et du pétrole (plus tard du gaz) importés - plombait la balance commerciale et aggravait les problèmes des houillères allemandes.

C'est dans ce contexte que le développement d'un programme électronucléaire allemand ambitieux émergea comme une nouvelle solution et comme la contrepartie nécessaire au soutien temporaire accordé aux charbonnages allemands, afin de compenser les surcoûts de la production charbonnière par une source d'énergie jugée peu onéreuse. Cette «économie des promesses ${ }^{11}$ » permettait de réconcilier en apparence les objectifs contradictoires de la politique économique. Elle était soutenue par des prévisions très optimistes concernant la croissance économique et énergétique mais aussi la baisse des coûts dans le nucléaire civil $^{12}$, qui formaient la base de la première programmation énergétique fédérale du 3 octobre 1973 (Energieprogramm). Afin de satisfaire l'augmentation projetée de la demande énergétique de $70 \%$ à l'horizon 1985, celle-ci préconisait le développement du pétrole et du nucléaire. La brusque augmentation des prix du pétrole à la fin de l'année 1973 conduisit à une réévaluation des risques de la dépendance énergétique croissante ${ }^{13}$, et précipita l'adoption d'une politique énergétique fédérale plus volontariste.

\section{"CoCoNuke » : un compromis fragile et temporaire}

La nouvelle stratégie, formulée dans de nouvelles programmations en 1974 puis en 1977, était résumée par la formule «CoCoNuke » (Düngen, 1993), désignant une concentration nouvelle sur le charbon (coal) - la première énergie domestique -, la maîtrise de la demande énergétique (conservation) et le nucléaire (nuke).

Dans le domaine du charbon, la signature du « contrat du siècle » (Jahrhundertvertrag) entre les énergéticiens, les grands clients industriels et l'industrie minière en 1977 reconnaissait à la houille allemande une place prioritaire dans l'approvisionnement énergétique du pays.

Pour couvrir les coûts, une redevance touchant les consommateurs finaux, le «denier du charbon» (Kohlepfennig), était introduite. Elle s'ajoutait à la fac-

\footnotetext{
${ }^{11}$ Pour cette notion, voir Joly (2010).

${ }^{12}$ La littérature académique allemande a introduit le terme de «surplus d'espoir » (Fraunholz et al., 2012, p. 24) pour caractériser ce penchant des prévisions à refléter la croyance de l'époque dans le progrès technique et économique.

${ }^{13}$ De $8 \%$ en 1960, la dépendance énergétique de l'Allemagne de l'Ouest a bondi à $60 \%$ en 1977.
} 
ture d'électricité et correspondait à un surcoût important, qui atteignit 8,25 \% de la facture ou 5,3 milliards de marks en 1990. Introduite par les électriciens à la suite des discussions dans les «tables rondes du charbon» (Kohlerunden), qui réunissaient régulièrement les acteurs privés et publics du secteur, acceptée par l'État et payée par les consommateurs, cette redevance marquait la reconnaissance du charbon comme élément intégral du mix énergétique allemand. Même après que la Cour constitutionnelle allemande l'a jugée anticonstitutionnelle (décision du 11 octobre 1994), mettant ainsi fin à la redevance, les gouvernements fédéraux ont continué à subventionner l'extraction du charbon allemand (BMWi/BMU, 2006).

Le programme nucléaire allemand était renforcé et son calendrier accéléré. La première loi sur l'atome date du 3 décembre 1959, quatre ans après que l'Allemagne eut regagné son droit de mener des recherches dans le domaine du nucléaire civil. Elle accordait un rôle central au gouvernement fédéral, cantonnant le rôle des Länder à l'autorisation et à la surveillance des réacteurs (Lippert, 2002, p. 287). Malgré cela, le nucléaire ne représentait que 3,7\% de la production d'électricité en Allemagne en $1970^{14}$.

Le «quatrième programme atomique» de novembre 1973 prévoyait de multiplier les capacités existantes par 30 à l'horizon 1985, principalement en facilitant l'investissement par des garanties étatiques et en relevant le montant des subventions accordées à l'industrie nucléaire. Ce programme était porté par un réseau dense formé d'industriels, de banques et de différentes branches de l'État et soutenu par des structures comme la Société allemande pour l'énergie atomique (Deutsche Gesellschaft für Atomenergie), l'Association d'ingénieurs allemands (Verband Deutscher Ingenieure) et le Forum atomique allemand (Deutsches Atomforum).

Le développement du programme nucléaire renforçait aussi la concentration du secteur. Sur le marché de la construction des réacteurs, la Kraftwerk Union, cofondée par Siemens et AEG, géant de l'électronique, était devenue l'entreprise dominante avec sa ligne de réacteurs Konvoi, qu'elle destinait également à l'exportation. En termes de production d'électricité, près de 1400 entreprises étaient présentes sur le marché en 1974, mais les deux majors RWE et Veba AG contrôlaient à elles seules $50 \%$ du marché, un chiffre qui montait à $73 \%$ pour les huit plus grandes entreprises du secteur (Nelkin/Pollak, 1981, p. 17). Cette organisation du secteur facilitait la coordination des acteurs et l'investissement, contribuant ainsi à l'essor rapide du nucléaire, qui couvrait $40 \%$ de la production d'électricité en Allemagne en 1985 (Herzig, 1992, p. 153 sq.) et qui était devenu la première source de production d'électricité, devant la houille et le lignite. Mais elle créait aussi un climat de confidentialité et d'opacité qui court-circuitait le processus démocratique, donnant ainsi prise à la critique antinucléaire naissante.

\footnotetext{
${ }^{14}$ Pour une analyse des politiques nucléaires allemandes, voir Meinert (1980, p. 43 sq.) et Herzig (1992, p. $153 s q$.).
} 
Enfin, les années 1970 marquaient aussi les débuts d'une politique de mâ̂trise de la demande. Une première loi fédérale du 22 juillet 1976 sur les économies d'énergie dans le bâti ${ }^{15}$ définissait un cadre pour cette politique. Elle a été précisée ensuite par des réglementations plus détaillées. Malgré cette activité réglementaire et des mesures symboliques qui, à l'instar des « dimanches sans voitures » (Autofreier Sonntag), ont marqué les esprits, les politiques de maîtrise de la demande restaient toutefois le parent pauvre des politiques énergétiques allemandes, subissant d'ailleurs, comme dans d'autres pays, une baisse d'intérêt après l'effondrement des cours du pétrole dans les années 1980.

\section{La vision d'un tournant énergétique}

Le programme électronucléaire allemand ne suscitait que peu de discussions dans les années 1950 (Fischer, 1992, p. 28), à une époque «d'euphorie de l'atome » (Atomeuphorie) où la technologie, soutenue par tous les partis représentés au Parlement, était considérée comme «le symbole même du progrès technique » (Herzig, 1992, p. 153).

Mais l'engouement pour l'atome commença à s'éroder dès la fin des années 1950, quand le public allemand découvrit que Franz Josef Strauss, ministre des Affaires atomiques avant de devenir ministre de l'Intérieur, poursuivait des plans visant à développer un programme nucléaire militaire, pourtant strictement interdit par les Alliés. Face à la levée de boucliers de la société civile et d'une large partie de la classe politique, il abandonna le projet. En réalité, la promulgation de la loi sur l'énergie atomique coïncidait déjà avec l'apogée du soutien des politiques nucléaires par l'opinion publique. Le déclin du soutien de l'opinion dans les décennies suivantes a tenu d'abord à des raisons économiques : le prix du pétrole baissait dans les années 1960, alors que les coûts pour la construction de centrales nucléaires s'avéraient considérablement plus importants que prévu (Fischer, 1992, p. 31). À cela s'ajoutaient des doutes croissants sur la capacité des autorités à maitriser les risques associés au nucléaire civil, renforcés par l'accident de Three Mile Island aux États-Unis en 1979.

Le désamour progressif des Allemands pour l'atome était aussi le fruit d'une politisation réussie de la question du nucléaire par le mouvement antinucléaire. Ainsi, le site de construction de la centrale nucléaire de Whyl, dans la vallée du Rhin proche de Fribourg, fut occupé dès 1975 par des agriculteurs et des activistes. Leur lutte marquait le début d'un nouveau mouvement social ${ }^{16}$ massif, qui essaima rapidement à travers l'Allemagne, à coup de manifestations et d'occupations de sites à Brokdorf (au Schleswig-Holstein), Grohnde (en

\footnotetext{
${ }^{15}$ Gesetz zur Einsparung von Energie in Gebäuden (EnEG), 1. Fassung vom 22.07.1976, BGB1. I 1976, p. 1873 .

${ }^{16}$ Pour une analyse des mouvements antinucléaires comme nouveaux mouvements sociaux, voir, parmi beaucoup d'autres, les ouvrages de Dieter Rucht $(1980$; 1994) et d'Alain Touraine $(1980 ; 1983)$.
} 
Basse-Saxe) et Kalkar (en Rhénanie du Nord-Westphalie), où 40000 personnes battaient le pavé en 1977 pour s'opposer à la construction du premier surgénérateur allemand. La même année, le gouvernement fédéral et le gouvernement régional de Rhénanie du Nord-Westphalie décidèrent d'explorer un site potentiel de stockage de déchets nucléaires à Gorleben, provoquant une mobilisation sans précédent qui culmina dans une manifestation de plus de 100000 personnes à Hanovre, la capitale régionale, le 31 mars 1979.

Ces manifestations étaient accompagnées par une longue série d'actions en justice qui parvenaient à retarder considérablement la construction de nouvelles centrales. Les procès donnaient aussi un forum à des experts proches du mouvement écologiste. Issus d'institutions d'éducation populaire comme la VHS Whyler Wald (créée en 1975) et d'instituts de recherche indépendants comme l'Öko-Institut (créé en 1975), ces experts alertaient l'opinion sur les dangers de l'énergie atomique et apportaient des informations sur les sources d'énergie alternatives comme les collecteurs solaires, les panneaux photovoltaïques ou les éoliennes.

C'est ce microcosme militant et expert qui donna naissance, en 1980, au rapport Energiewende, «tournant énergétique », travail fondateur concernant la perspective d'une transition énergétique en Allemagne (Krause et al., 1980). Publiée par l'Öko-Institut, l'étude est axée autour d'une vision nouvelle : découpler la croissance économique et la consommation d'énergie et sortir simultanément du nucléaire et du pétrole en s'appuyant sur les énergies renouvelables et la maîtrise de la demande, tout en faisant appel, au moins temporairement, au charbon pour couvrir les besoins énergétiques de l'économie allemande.

Le rapport, qui trouva d'abord peu d'écho dans la presse allemande, fut ensuite relayé par le rapport de l'enquête parlementaire sur « la future politique nucléaire » (1979-1982) (Altenburg, 2010). S'il a réussi à profondément changer le regard des Allemands sur les politiques énergétiques - et à donner son nom au tournant énergétique allemand vingt ans plus tard - c'est parce que, d'abord, le rapport ne se contente pas d'avertir des dangers de l'énergie atomique mais qu'il propose, pour la première fois, une vision cohérente d'un futur énergétique alternatif.

Ensuite, le vent politique et sociétal commençait à tourner au début des années 1980. Selon les sondages de l'institut TNS Emnid, la part de la population en faveur du programme nucléaire était passée de $40 \%$ en 1974 à $30 \%$ en 1985 et à seulement $17 \%$ en 1988, après la catastrophe nucléaire de Tchernobyl (Fischer, 1992, p. 32). Enfin, le rapport a initié un mouvement local, avec la formation de centaines de «comités de tournant énergétique », Energiewendekomitees, qui ont multiplié les scénarios de transition au niveau communal et donné un fort ancrage territorial à la vision de l'Öko-Institut.

Parallèlement à l'évolution de l'opinion publique, les sociaux-démocrates du SPD, qui avaient pourtant été au gouvernement fédéral dans différentes coalitions gouvernementales durant toute la période de construction du parc nu- 
cléaire allemand, opérèrent un revirement sur la question nucléaire. Initialement favorable à l'atome, le parti était de plus en plus exposé à la gronde des milieux charbonniers qui redoutaient, non sans raison, que l'ambitieux programme nucléaire ne mît en danger le soutien aux charbonnages allemands (Matthöfer, 1976). En proie aux critiques des antinucléaires et des syndicats, le parti craignait alors de perdre une partie importante de son électorat.

À la fin des années 1970, qui avaient vu une multiplication par dix de la capacité nucléaire ouest-allemande, les sociaux-démocrates révisèrent leur position dans une série de congrès nationaux. Cette évolution culmina au congrès de Hambourg en 1977, où le SPD adopta la «doctrine des deux options » (Ehmke, 1994, p. 290). Celle-ci prescrivait le maintien des centrales existantes et en construction, mais prévoyait aussi de s'abstenir de toute nouvelle construction et de combler les besoins énergétiques par une politique volontariste d'économies d'énergie et une politique de préférence pour le charbon, surtout allemand.

Mais même cette ligne qui se voulait équilibrée s'avéra rapidement intenable. Après le passage du parti dans l'opposition parlementaire en octobre 1982, il assistait, impuissant, à l'entrée au Bundestag des Verts allemands en 1983 et à leur participation au gouvernement régional de Hesse en 1985. Alors qu'une alternative résolument antinucléaire émergeait donc à gauche du SPD, la catastrophe de Tchernobyl en avril 1986 poussa le parti à compléter sa mue et à se prononcer pour la sortie du nucléaire, appelant désormais à un arrêt des centrales existantes « en dix ans » (voir par exemple Bundestag, 1987).

\section{Un tournant énergétique favorisé par une géographie de l'énergie particulière}

L'accident de Tchernobyl a scellé le sort de l'énergie nucléaire en Allemagne : même le gouvernement conservateur de Helmut Kohl, pourtant pronucléaire, ne la voyait plus que comme une «technologie de transition » (Brückentechnologie) sur la voie d'un système énergétique plus durable. À la différence de la situation française (Chateauraynaud, 2011), l'alerte climatique n'a d'ailleurs pas renversé la situation argumentative en faveur des partisans d'une relance du nucléaire.

Pour comprendre ce décalage, il faut revenir sur l'histoire particulière de la construction du changement climatique comme problème public en Allemagne, et en particulier à la commission parlementaire sur « la précaution pour la protection de l'atmosphère »(1986-1990). En présentant trois scénarios énergétiques, avec et sans nucléaire, et en démontrant que tous étaient compatibles avec une politique ambitieuse de décarbonation, cette commission a réussi à créer un consensus large et partagé sur les bases scientifiques de l'alerte sur le réchauffement global et sur la nécessité de prendre des mesures de précaution ambitieuses, tout en «découplant» la question climatique de la question nucléaire (Aykut, 2015). 
Ses conclusions ont fondé la décision unilatérale du gouvernement allemand de réduire ses émissions de $25 \%$ entre 1990 et 2005 . Le gouvernement allemand s'investissait aussi sur le plan international, revendiquant un rôle de précurseur. Il devint notamment l'hôte de la première conférence des parties de la convention climat à Berlin en 1995. C'est donc dès cette période que se dessinait un autre objectif important du futur tournant énergétique allemand qui vise, en plus de la sortie du nucléaire, une décarbonation profonde de l'économie. Un troisième pilier du tournant futur, le soutien aux énergies renouvelables, est entré dans la législation allemande dès le 7 décembre 1990, quand une loi (Stromeinspeisungsgesetz) a introduit un tarif d'achat garanti pour les énergies renouvelables. Celui-ci a profité surtout à l'éolien, faisant de l'Allemagne le pays précurseur dans ce domaine dès le milieu des années 1990.

Quels sont les éléments qui permettent alors de rendre intelligible cette trajectoire allemande particulière dans le domaine énergétique, qui s'était dessinée bien avant l'arrivée au pouvoir du gouvernement associant le SPD et les Verts en 1998 ?

Au lieu d'une lecture répandue qui insiste sur une supposée sensibilité particulière des Allemands envers la nature qui les prédisposerait davantage à l'écologie politique, nous souhaitons insister ici sur deux facteurs ${ }^{17}$ sociopolitiques qui tiennent plus aux circonstances spécifiques de l'histoire allemande récente qu'à une quelconque prédisposition culturelle, et qui renvoient :

- à l'émergence d'un discours de «modernisation écologique » permettant de concilier politiques industrielles et politiques de protection de l'environnement ;

- à une géographie de l'énergie particulière qui a favorisé l'émergence de coalitions d'acteurs alternatives.

Dès le début des années 1980, un ensemble de travaux, plus tard rangés sous la bannière de la «modernisation écologique ${ }^{18}$ » insistaient sur la compatibilité entre le développement économique et la protection de l'environnement. Ce débat connaissait un succès important en Allemagne parce qu'il coïncidait avec l'essor d'une nouvelle branche industrielle, celle des «industries de protection de l'environnement ».

En effet, le début des années 1980 était marqué par l'affaire des pluies acides. Les normes et les réglementations introduites sur ce dossier ont fait émerger une industrie dotée d'une expertise spécifique dans la lutte contre les pollutions, avec une capacité de surveillance et de réduction des émissions. Weidner et Mez (2008, p. 360) invoquent la création d'une «dépendance au

\footnotetext{
${ }^{17}$ Nous avons limité les facteurs explicatifs à deux par souci d'économie d'espace. Il faudrait ajouter au moins deux éléments que nous avons décrits ailleurs (Aykut, 2012, p. 353 sq.) : d'une part, la jonction qui s'est opérée entre mouvement antinucléaire et mouvement pour la paix (manifestations anti-Pershing II) et, d'autre part, les caractéristiques structurelles du système politique allemand, qui ont favorisé l'institutionnalisation des Verts allemands.

${ }^{18}$ Pour les origines de cette notion, on pourra se référer aux travaux de Huber (1982) et Jänicke (1985).
} 
sentier » positive : l'épisode montrait qu'il était possible de mettre en œuvre des politiques ambitieuses de protection de l'environnement sans que cette politique n'entraîne de conséquences économiques négatives. Bien au contraire, plusieurs études montraient des effets positifs sur l'emploi, l'innovation et la modernisation des branches concernées.

Selon les statistiques de l'Organisation de coopération et de développement économiques (OCDE), l'industrie de protection de l'environnement allemande employait 320000 personnes à la fin des années 1980, avec un chiffre d'affaires de quelque 40 milliards de marks et une orientation à l'export, avec $40 \%$ de ses produits destinés à l'étranger (OCDE, 1993). Cette expansion industrielle dans le domaine de l'environnement se poursuivit dans les années 1990. En 1998, environ 3,6\% des employés allemands (1,3 millions de personnes) travaillaient dans l'industrie de l'environnement, ce qui faisait de l'Allemagne le pays de l'OCDE où cette branche était la plus développée et la plus dynamique (BMU, 2002). Par conséquent, l'environnement cessait d'être une niche politique pour devenir un facteur important de compétition économique, avec une capacité de lobbying qui n'avait rien à envier aux autres secteurs industriels.

La géographie politique de l'énergie en Allemagne est le deuxième facteur ayant favorisé le changement des politiques énergétiques.

En raison de la structure fédérale et polycentrique de l'Allemagne d'aprèsguerre, les politiques énergétiques allemandes n'ont jamais procédé d'une mise en œuvre centralisée et homogène mais elles ont pris la forme d'une négociation complexe et continue entre des acteurs divers, avec une forte capacité d'obstruction des communes et des États fédérés s'ils considéraient qu'ils n'avaient pas été associés à la prise de décision.

À ceci s'ajoute l'existence de coalitions politico-géographiques, qui ont constitué une faille dans le front de soutien à l'atome. Ces coalitions trouvent leur origine dans la distribution des ressources d'énergie sur le territoire allemand, avec une forte concentration du charbon dans l'ouest et l'est du pays, tandis que le nord et le sud ne sont que peu dotés en ressources fossiles. Si les régions charbonnières font donc naturellement la part belle aux centrales à charbon pour produire de l'électricité, les autres ont plutôt misé sur le nucléaire.

Une véritable géopolitique de l'énergie s'est alors dessinée : ainsi, la Rhénanie du Nord-Westphalie, dans le nord-ouest de l'Allemagne, région la plus peuplée du pays dont l'économie a dépendu pendant longtemps du bassin charbonnier de la Ruhr, était un bastion des sociaux-démocrates. Soutenu par les puissants syndicats miniers, le parti y fut au pouvoir sans interruption durant 40 ans, entre 1966 et 2005. En revanche, un seul des 20 réacteurs nucléaires allemands d'une puissance de plus de 500 MW était localisé dans cet État fédéré (centrale de Würgassen), alors que les deux grands États fédérés du Sud, le Bade-Wurtemberg et la Bavière en comptaient respectivement quatre et cinq. La Bavière abritait aussi le premier réacteur de recherche (Munich, mis en service en 1957) et le premier réacteur raccordé au réseau (Kahl, en 1962), ainsi que le 
plus puissant réacteur construit en Allemagne, Isar 2, avec une puissance brute de 1485 MW. Les deux États fédérés du Sud sont des remparts pour les conservateurs de l'union chrétienne-démocrate et de l'union chrétienne-sociale (CDU/CSU), qui ont dirigé le Bade-Wurtemberg entre 1953 et 2011 et gouvernent la Bavière depuis 1957 (!)

Au prisme de cette géographie politique de l'énergie, le compromis énergétique des années 1970-1980 apparaît comme un équilibre fragile entre des coalitions concurrentes composées de partis politiques, d'États fédérés, d'entreprises énergétiques, de syndicats, d'associations patronales et de formes diverses d'énergie, qui se manifestent dans des forums particuliers comme les «Kohlerunden ${ }^{19} »$ et l' «Atomforum ». Cette fragmentation de l'espace politique et social autour de la question énergétique a permis au mouvement antinucléaire de nouer des alliances de circonstance, favorisant ainsi l'instauration progressive d'une alternative aux politiques publiques établies.

LE REGARD HISTORIQUE PERMET DONC DE MONTRER que le changement récent des politiques énergétiques allemandes, loin d'être le fruit d'une décision prise «à chaud » après la catastrophe de Fukushima en 2011, découle de transformations d'abord graduelles qui ont leur origine dans l'Allemagne des années 1970 et 1980. Porté par un mouvement social puissant, le changement a été favorisé par un ensemble de facteurs sociopolitiques propres à l'Allemagne d'après-guerre : importance de l'échelle locale et régionale dans les politiques énergétiques ; secteur énergétique entre libre marché et participations étatiques ; géographie de l'énergie particulière, rendant difficile une hégémonie de l'électronucléaire comme en France ; enfin, émergence d'une «alternative de politique publique » avec le discours de modernisation écologique et l'idée d'une transformation écologique du système énergétique. Initié par des instituts de recherche proches du mouvement antinucléaire, ce discours a été repris dans le débat public et par une partie de l'administration, avant de fournir un cadre conceptuel au tournant allemand à la fin des années 1990.

Dans un contexte actuel marqué par une généralisation, au niveau européen comme au niveau mondial, de l'injonction à la transformation profonde des politiques énergétiques nationales pour répondre aux multiples crises qui secouent le secteur (climatique, du pétrole, écologique, géopolitique...), ce retour sur le temps long du changement permet aussi de questionner l'apparente convergence de situations nationales que sous-entend l'usage indifférencié de la notion de «transition énergétique ». Ce n'est qu'au prisme de cette histoire longue des politiques énergétiques qu'il paraît possible de comparer des trajectoires nationales, de comprendre pourquoi certaines transformations semblent irréversibles alors que d'autres restent contestées, voire d'envisager la transposition de certains éléments, enseignements, ou instruments d'une transition à une autre.

\footnotetext{
${ }^{19}$ Ces « tables rondes du charbon » réunissent régulièrement acteurs économiques, syndicats et État pour discuter des mesures de soutien aux charbonnages allemands.
} 


\section{Indications bibliographiques}

Altenburg C., Kernenergie und Politikberatung - Die Vermessung einer Kontroverse, VS Verlag für Sozialwissenschaften, Wiesbaden, 2010

AyKuT S. C., Comment gouverner un 'nouveau risque mondial' ? - La construction du changement climatique comme problème public à l'échelle globale, européenne, en France et en Allemagne, Thèse de doctorat en sciences sociales soutenue le 30 mai 2012, École des Hautes Études en Sciences Sociales (EHESS), Paris, 2012

AYKuT S. C., «Energy Futures from the Social Market Economy to the Energiewende: The Politicization of West German Energy Debates, 1950-1990 », in ANDERSSON J., RINDZEVIČIŪTĖ E. (dir.), The Struggle for the Long Term in Transnational Science and Politics - Forging the Future, Routledge, Londres, 2015, p. 63 91.

BEYER J., « Deutschland AG a.D.: Deutsche Bank, Allianz und das Verflechtungszentrum großer deutscher Unternehmen », MPIfG Working Paper, $\mathrm{n}^{\circ}$ 02/4, 2002, p. [n.c.]

BIER C., Regulierter oder Verhandelter Zugang zum Stromnetz? - Eine ökonomische Analyse unter Berücksichtigung imperfekter Aufsichtsbehörden, Verlag Dr. Kovač, Hambourg, 2002

BUNDESMINISTERIUM FÜR UMWELT, NATURSCHUTZ, BAU UND REAKTORSICHERHEIT (BMU), Umweltbericht 2002 - Bericht über die Umweltpolitik der 14. Legislaturperiode; Ökologisch, modern, gerecht; die ökologische Modernisierung von Wirtschaft und Gesellschaft, Bonn, 2002

BUNDESMINISTERIUM FÜR WIRTSCHAFT UND TECHNOLOGIE (BMWI), BUNDESMINISTERIUM FÜR UMWELT, NATURSChUTZ, BAU UND REAKTORSICHERHEIT (BMU), Energieversorgung für Deutschland - Statusbericht für den Energiegipfel am 3. April 2006, Berlin, 2006

BUNDESTAG, « Kohlevorrangpolitik - Antrag der Fraktion der SPD, », Drucksachen, n¹1/958, 14/10/1987, p. $1-8$

Chateauraynaud F., « Sociologie argumentative et dynamique des controverses : l'exemple de l'argument climatique dans la relance de l'énergie nucléaire en Europe », A contrario, n ${ }^{\circ}$ 16, 2011/2, p. 131-150

DünGEN H., «Zwei Dekaden deutscher Energie- und Umweltpolitik: Leitbilder, Prinzipien und Konzepte », in Hohensee J., SALEWSKI M. (dir.), Energie - Politik - Geschichte - Nationale und internationale Energiepolitik seit 1945, Franz Steiner Verlag, Stuttgart, 1993, p. [n.c.]

Енмке H., Mittendrin - Von der Großen Koalition zur Deutschen Einheit, Rowohlt, Berlin, 1994

EVRARD A., «Les énergies renouvelables et l'électricité : à propos d'un conflit entre un secteur et une alternative de politique publique », Écologie et Politique, n49, 2014, p. 67-80

FisCHER W. (dir.), Die Geschichte der Stromversorgung, Verlags- und Wirtschaftsgesellschaft der Elektrizitätswerke, Francfort-sur-le-Main, 1992

Fraunholz U., HänSeroth T., Woschech A., « Hochmoderne Visionen und Utopien. Zur Transzendenz technisierter Fortschrittserwartungen », in U. Fraunholz, WoscheCH A. (dir.), Technology Fiction Technische Visionen und Utopien in der Hochmoderne, Transcript, Bielefeld, 2012, p. 11-24

Gammelin C., Hamann G., Die Strippenzieher: Manager, Minister, Medien - Wie Deutschland regiert wird, Econ, Berlin, 2005

Herzig T., «Wirtschaftsgeschichtliche Aspekte der deutschen Elektrizitätsversorgung 1880 bis 1990 », in FisCHER W. (dir.), Die Geschichte der Stromversorgung, Verlags- und Wirtschaftsgesellschaft der Elektrizitätswerke, Francfort-sur-le-Main, 1992, p. 122-166

HubER J., Die verlorene Unschuld der Ökologie-Neue Technologien und superindustrielle Entwicklung, Fischer, Francfort-sur-le-Main, 1982

HuSKe J., Die Steinkohlezechen im Ruhrrevier - Daten und Fakten von den Anfängen bis 2005, $3^{\mathrm{e}}$ édition, Selbstverlag des Deutschen Bergbau-Museums, Bochum, 2006 
JäNICKE M., «Preventive Environmental Policy as Ecological Modernisation and Structural Policy», WZB/IIUG Discussion Papers, ${ }^{\circ} 85-2,1985$, p. [n.c.]

Joly P.-B., « On the Economics of Techno-scientific Promises », in AKrich M., Barthe Y., Muniesa F., Mustar P. (dir.), Débordements - Mélanges offerts à Michel Callon, Presse des Mines, Paris, 2010, p. 203222

KLEINWÄChTER K., «Das „Eiserne Pentagramm“ - Strommarktregulierung in Deutschland », in KLEINWÄCHTER L. (dir.), Deutsche Energiepolitik, Brandenburgische Landeszentrale für politische Bildung, Potsdam, 2007, p. 65-79

Krause F., Bossel H., Müller-Reissmann K.-F., Energie-Wende: Wachstum und Wohlstand ohne Erdöl und Uran - Ein Alternativ-Bericht des Öko-Instituts, Fischer, Francfort-sur-le-Main, 1980

KRISP A., Der deutsche Strommarkt in Europa - Zwischen Wettbewerb und Klimaschutz, Thèse de doctorat en sciences humaines et sociales, université Justus-Liebig, Giessen, 2007

LIPPERT M., Energiewirtschaftsrecht - Gesamtdarstellung für Wissenschaft und Praxis, Deutscher Wirtschaftsdienst, Cologne, 2002

MATthöFER H., Interviews und Gespräche zur Kernenergie, C.F. Müller, Karlsruhe/Heidelberg, 1976

MEINERT J., Strukturwandlungen der westdeutschen Energiewirtschaft - Die Energiepolitik der Bundesregierung von 1950 bis 1977 unter Berücksichtigung internationaler Dependenzen, Metzner, Francfort-sur-leMain, 1980

Meyer-Abich K.-M., Dickler R.A., «Energy Issues and Policies in the Federal Republic of Germany », Annual Review of Energy, vol. 7, novembre 1982, p. 221-259

Nelkin D., Pollak M., The Atom Besieged - Extraparliamentary Dissent in France and Germany, MIT Press, Cambridge, MA, 1981

OCDE, Données OCDE sur l'Environnement - Compendium 1993, Paris, 1993

Pfaffenberger W., Scheele U., STröbele W., Windelberg J., Auswirkungen des EG-Binnenmarkts für Energie auf die räumliche Energieversorgung - Studie im Auftrag des Bundesministers für Raumordnung, Bauwesen und Städtebau, Schulz-Kirchner, Idstein, 1992

RuCHT D., Von Wyhl nach Gorleben - Bürger gegen Atomprogramm und nukleare Entsorgung, Beck, Munich, 1980

RuchT D., Modernisierung und neue soziale Bewegungen - Deutschland, Frankreich und USA im Vergleich, Campus Verlag, Francfort-sur-le-Main, New York, 1994

STIER B., Staat und Strom - Die politische Steuerung des Elektrizitätssystems in Deutschland 1890-1950, Verlag Regionalkultur, Ubstadt-Weiher, 1999

Touraine A., Anti-Nuclear Protest - The Opposition to Nuclear Energy in France, Cambridge University Press, Cambridge, 1983

Touraine A., Hegedus Z., Dubet F., Wieviorka M., La prophétie anti-nucléaire, Seuil, Paris, 1980

Weidner H., Mez L., «German Climate Change Policy: A Success Story With Some Flaws », The Journal of Environment \& Development, vol. 17, $\mathrm{n}^{\circ}$ 4, décembre 2008, p. 356-378. 

- II -

LA TRANSITION ÉNERGÉTIQUE ET L'ENERGIEWENDE 



\section{Comparatif des politiques énergétiques : les voies française et allemande pour la transformation du système énergétique ${ }^{1}$}

\section{Severin FISCHER}

Avec l'Allemagne et la France, ce sont deux grands États membres de l'Union européenne (UE) qui ont entrepris de transformer en profondeur leur système énergétique. Dans ce processus, non seulement la nécessité de gérer l'approvisionnement énergétique de manière plus respectueuse de l'environnement et du climat, mais aussi les contextes nationaux et les tendances sociopolitiques jouent un rôle important. C'est pourquoi cela vaut la peine de jeter un coup d'œil sur les points communs et les différences entre les deux pays en termes de débat national et d'objectifs de la transition énergétique.

\section{Des objectifs communs par des voies différentes}

En comparant les deux stratégies de transformation de la politique énergétique en Allemagne et en France, on constate que «Energiewende » et «transition énergétique » présentent un nombre étonnant d'objectifs concordants et parfois coïncidents. Les décisions prises par l'Allemagne en 2011 formulent un cadre complet de jalons à court, moyen et long terme pour réduire les émissions de gaz à effet de serre, renforcer les énergies renouvelables, améliorer l'efficacité énergétique et traiter toute une série d'autres sujets. On retrouve des éléments similaires dans le projet de loi «transition énergétique pour la croissance verte » présenté en juillet 2014 par la ministre française de l’Écologie, du Développement durable et de l'Énergie, Ségolène Royal. L'Assemblée nationale a adopté cette loi en première lecture ${ }^{2}$. Même en considérant le sujet politiquement sensible de la réduction du nucléaire dans la production d'électricité, les deux gouvernements ont adopté une démarche de transformation tout aussi exigeante, qui nécessite encore toutefois l'approbation finale du Parlement en France. Les objectifs comparables des deux programmes énergétiques ne doivent cependant pas mener à la conclusion erronée que les évolutions politiques sous-jacentes, les motivations des acteurs politiques et l'opinion publique sur les questions de politique énergétique s'accordent également.

\footnotetext{
${ }^{1}$ Ce texte constitue la version condensée (en juin 2015) de l'article suivant : Franca Diechtl, Severin Fischer, «Unter neuen Vorzeichen - Transformationsprozesse und Kooperationsmodelle in den deutsch-französischen Energiebeziehungen » [en ligne], DGAPanalyse, $\mathrm{n}^{\circ}$ 5, mai 2015, p. 1-12. Disponible sur : https://dgap.org/de/ article/getFullPDF/26775 [consulté le 02/10/2015], NdE.

${ }^{2}$ Cf. Severin Fischer, «Frankreichs energiepolitische Reformagenda. Grüne Wachstumsimpulse und strategische Ambivalenzen » [en ligne], SWP-Aktuell, $\mathrm{n}^{\circ}$ 61, octobre 2014, p. 1-4. Disponible sur : http://www.swpberlin.org/fileadmin/contents/products/aktuell/2014A61_fis.pdf [consulté le 06/05/2015].
} 


\section{Tableau 1 : Liste des objectifs stratégiques de l'Allemagne (Energiewende de juin 2011 ou Energiekonzept de 2010) et de la France (projet de loi « transition énergétique » de juillet 2014)}

\begin{tabular}{|c|c|c|c|}
\hline & \\
\hline & Objectif & Allemagne & France \\
\hline \multirow{3}{*}{$\begin{array}{l}\text { Réduction des émissions } \\
\text { de gaz à effet de serre } \\
\text { (par rapport à 1990) }\end{array}$} & 2020 & $-40 \%$ & $-20 \%$ \\
\hline & 2030 & $-55 \%$ & $-40 \%$ \\
\hline & 2050 & -80 à $-95 \%$ & $-75 \%$ \\
\hline \multirow{3}{*}{$\begin{array}{l}\text { Part des énergies renouvelables } \\
\text { dans la consommation } \\
\text { d'énergie finale }\end{array}$} & 2020 & $18 \%$ & $23 \%$ \\
\hline & 2030 & $30 \%$ & $32 \%$ \\
\hline & 2050 & $\min .60 \%$ & - \\
\hline \multirow{3}{*}{$\begin{array}{l}\text { Part des énergies renouvelables } \\
\text { dans la consommation brute } \\
\text { d'électricité }\end{array}$} & 2020 & $35 \%$ & - \\
\hline & 2030 & $50 \%$ & - \\
\hline & 2050 & $\min .80 \%$ & - \\
\hline $\begin{array}{l}\text { Réduction de la part } \\
\text { des énergies fossiles }\end{array}$ & 2030 & - & $-30 \%$ \\
\hline \multirow{2}{*}{ Économies d'énergie } & 2020 & $-20 \%$ & $-20 \%$ \\
\hline & 2050 & $-50 \%$ & $-50 \%$ \\
\hline $\begin{array}{l}\text { Réduction de la part de l'énergie } \\
\text { atomique par rapport à } 2010^{3}\end{array}$ & $\begin{array}{c}2022 \\
\text { (ou 2025) }\end{array}$ & $\begin{array}{r}-22 \% \\
\text { (env. }-162 \mathrm{TWh})\end{array}$ & $\begin{array}{r}-28 \% \\
\text { (env. }-154 \mathrm{TWh})\end{array}$ \\
\hline Électromobilité & & $\begin{array}{l}2020 \text { : } 1 \text { million de } \\
\text { véhicules électriques } \\
\text { (ou hybrides) }\end{array}$ & $\begin{array}{l}2030: 7 \text { millions de } \\
\text { stations de recharge }\end{array}$ \\
\hline
\end{tabular}

Source : Franca Diechtl, Severin Fischer, «Unter neuen Vorzeichen - Transformationsprozesse und Kooperationsmodelle in den deutsch-französischen Energiebeziehungen », DGAPanalyse, $\mathrm{n}^{\circ}$ 5, mai 2015, p. 4. Disponible sur : https://dgap.org/de/article/getFullPDF/26775 [consulté le 02/10/2015].

Le tournant énergétique allemand ne peut s'expliquer, a posteriori, que si on le comprend comme le résultat d'un processus qui s'étend sur plusieurs décennies pendant lesquelles la société a débattu des questions de politique énergétique. À ce jour, cette préoccupation pour la politique énergétique est profondément ancrée dans les groupes sociaux et les partis ${ }^{4}$. C'est un processus marqué par le grand professionnalisme des mouvements écologistes allemands, l'accent mis sur le rôle de l'énergie atomique en tant qu'élément de discussion central et l'orientation scientifique du débat allemand. Avant même la catastrophe de

\footnotetext{
${ }^{3}$ Entre parenthèses: estimation de la réduction absolue de la production annuelle d'électricité d'origine nucléaire, à production d'électricité constante.

${ }^{4}$ Cf. Joachim Radkau, Die Ära der Ökologie. Eine Weltgeschichte, Bundeszentrale für politische Bildung, Bonn, 2011.
} 
Fukushima, des décisions politiques avaient été prises pour une transformation écologique du système énergétique : les événements de mars 2011 n'ont fait que les confirmer plus ouvertement. La part relativement faible de l'énergie atomique dans la production d'électricité en Allemagne et la progression fulgurante des énergies renouvelables ont permis d'engager très tôt un « débat sur la substitution » qui donnait à penser que l'intégralité du parc de centrales nucléaires pouvait être remplacée de manière rentable. Contrairement à l'intégration historique des mouvements de protestation sociale dans le système politique allemand, en France le mouvement antinucléaire est toujours resté relativement restreint en termes d'effectifs et n'a eu qu'un accès limité au système politique, aux ressources et donc au grand public. Le système électoral et les partis de la $\mathrm{V}^{\mathrm{e}}$ République ont empêché les mouvements environnementaux déjà très faibles de conquérir les institutions. De plus, le nucléaire brandi comme un étendard patriotique, symbole de puissance militaire et économique, et la volonté politique d'assurer l'indépendance énergétique de la France ont renforcé le consensus général en faveur du maintien de cette énergie.

\section{Politique environnementale : des interprétations différentes}

Le débat public sur les futures lois du «Grenelle de l'environnement » entamé en 2007 sous la présidence de Nicolas Sarkozy témoignait aussi d'un changement de l'opinion publique en matière de protection de l'environnement et de lutte contre le changement climatique ${ }^{5}$. Alors qu'en Allemagne, c'étaient surtout le mouvement antinucléaire et un lobby grandissant des énergies renouvelables qui se faisaient les plus visibles, le débat français portait plus largement sur des thèmes tels que le développement durable et la sobriété énergétique. En plus d'un intérêt croissant pour les problématiques motivées par la politique environnementale, la politique énergétique française doit faire face depuis plusieurs années à des défis systémiques. On peut notamment mentionner le vieillissement du parc de centrales nucléaires, majoritairement construites dans les années 1970-1980, la prédominance d'un unique type de réacteurs dans ce parc, ce qui entraîne un risque élevé en cas de défaillance des structures ou des matériaux dans un grand nombre d'installations similaires, et enfin l'augmentation de la consommation d'électricité ainsi que la thermosensibilité du système électrique français, poussé aux limites de ses capacités pendant les mois d'hiver'.

Le fait que le président François Hollande ait intégré dans son programme électoral de 2012 l'annonce d'une réforme de la politique énergétique ne

\footnotetext{
${ }^{5}$ Les lois du « Grenelle de l'environnement » ont été adoptées en deux temps en 2007 et 2008, alors que JeanLouis Borloo était ministre de l'Environnement. Elles portaient principalement sur le développement durable, la biodiversité, la réduction des émissions de gaz à effet de serre et des mesures d'efficacité énergétique.

${ }^{6}$ Cf. David Buchan, The French Disconnection: Reducing the nuclear share in France's energy mix [en ligne], Oxford Institute for Energy Studies, Oxford, 2014 (OIES Paper; SP 32). Disponible sur: http://www.oxfordenergy.org/wpcms/wp-content/uploads/2014/01/SP-32.pdf [consulté le 06/05/2015].
} 
résultait donc pas seulement de la volonté de courtiser un électorat aux orientations écologiques ou d'intégrer le parti des Verts dans le gouvernement, ni même de la catastrophe nucléaire de Fukushima, mais reflétait en grande partie la nécessité de réforme inhérente au système. Avec le lancement du débat sur la transition énergétique, le gouvernement fraîchement nommé par le président Hollande a adopté une approche d'intégration qui avait déjà fait ses preuves dans le cadre des processus du Grenelle, réunissant de nombreux acteurs de différents groupes de la société et des régions du pays ${ }^{7}$. Il s'agissait là toutefois d'une rupture vis-à-vis de la tradition technocratique centralisée en matière de politique énergétique, ce qui n'a pas suscité une sympathie démesurée au sein des ministères concernés. De ce point de vue, on peut désormais voir un parallèle avec le processus de transformation de la politique énergétique en Allemagne, qui a toujours été profondément ancré dans la structure fédéraliste du pays - ancrage encore renforcé avec la nouvelle répartition des flux financiers prévue par la loi sur les énergies renouvelables. Alors que la France fait ses premiers pas vers une participation des acteurs régionaux dans la politique énergétique, le rôle fort des communes et des Länder apparaît comme une caractéristique spécifique du tournant énergétique allemand. Ces dernières années, celui-ci s'est transformé en défi pour l'organisation du système allemand tout entier, qui est de plus en plus souvent confronté à des questions de solidarité en matière d'extension du réseau ou d'ajout de capacités de production dans le domaine des énergies renouvelables. Même le débat actuel en Allemagne sur le rôle futur du charbon dans le mix énergétique n'a pratiquement aucun écho en France, en raison du rôle marginal de cette source d'énergie dans le pays. On constate aussi une différence fondamentale du point de vue du rôle du secteur de l'électricité dans la politique climatique, puisque celui-ci est responsable d'environ 19 millions de tonnes de $\mathrm{CO}_{2}$ en 2014 en France, du fait de la part élevée du nucléaire, contre 301 millions de tonnes en Allemagne ${ }^{8}$.

De nettes différences entre les processus de transformation des deux pays se révèlent également en matière de prise de décision et de perception de leur durabilité dans les systèmes politiques respectifs. Alors que les principales décisions sur le tournant énergétique allemand ont été prises au printemps 2011 avec un large consensus de tous les partis représentés au Bundestag ainsi que des ministres-présidents des Länder, la transition énergétique reste un sujet d'affrontement politique en France. À ce jour en Allemagne, le consensus porte sur la réalisation du tournant énergétique avec ses principales étapes de transformation, la sortie du nucléaire d'ici 2022 et le développement des énergies renouvelables. Il ne subsiste de controverse que sur l'organisation des instru-

\footnotetext{
${ }^{7}$ Cf. Andreas Rüdinger, Die Energiewende in Frankreich. Aufbruch zu einem neuen Energiemodell? [en ligne], Friedrich-Ebert-Stiftung, Berlin, 2014 (Internationale Politikanalyse). Disponible sur : http://library. fes.de/pdf-files/id/10960.pdf [consulté le 06/05/2015].

${ }^{8}$ Les chiffres sont tirés des données du gestionnaire du réseau de transport RTE en France, et du think tank Agora Energiewende ou de l'Office fédéral de l'environnement en Allemagne.
} 
ments politiques et les mesures d'infrastructures nécessaires. En France, en revanche, l'intégralité du projet pourrait faire l'objet d'une révision en cas de changement de gouvernement. Dès le débat au Sénat, on a pu constater que la forte réduction de la part du nucléaire dans la production d'électricité et les objectifs d'efficacité énergétique, tels qu'ils étaient présentés, faisaient débat entre les partis. Même au sein de la gauche française, il existe des points de vue divergents à cet égard, de sorte qu'il est impossible de parler d'un large « consensus sur la transition énergétique » tel qu'il existe en Allemagne.

De part et d'autre du Rhin, une question centrale se pose sur le financement des stratégies respectives de transformation. Il existe déjà d'importantes obligations de financement dans les deux pays, mais elles sont réparties différemment entre consommateurs finaux, budget public et entreprises (publiques). En France, les tarifs réglementés de l'électricité et la nécessité de moderniser le parc nucléaire pèsent dans la balance. En Allemagne, en revanche, les obligations résultant de la redevance sur les énergies renouvelables nécessiteront aussi à l'avenir d'importants transferts de la part des consommateurs d'électricité. Le principal facteur d'incertitude réside toutefois, pour les deux pays, dans le financement futur des mesures d'efficacité énergétique, en particulier dans le secteur du bâtiment, pour atteindre les objectifs politiques. Le financement de l'électromobilité reste également un poste en grande partie non couvert dans les deux budgets. Certes, l'Allemagne a conçu un modèle de financement par la banque publique Kreditanstalt für Wiederaufbau (KfW), alors qu'en France tout reste à commencer. Cependant, l'Allemagne n'arrivera pas à ses fins sans financements publics. En ce qui concerne la situation budgétaire et le respect de la limite des $3 \%$ de déficit public par rapport au produit intérieur brut (PIB) fixée par Bruxelles, il semble que l'Allemagne bénéficie d'une flexibilité accrue?

\section{État et marché}

Enfin, une étude comparative des voies choisies par l'Allemagne et la France pour cette transformation ne peut se terminer sans un coup d'œil sur le cadre économique du secteur énergétique, qui présente sans aucun doute d'importantes différences entre les deux pays. Pour les deux gouvernements, le cadre de la politique énergétique constitue un facteur déterminant pour les performances ultérieures de leur économie respective. Ils semblent s'accorder sur la nécessité de développer des technologies innovantes et de soutenir les entreprises qui s’y emploient. Cependant, le rôle de l'État dans ce processus reste l'objet de divergences. Tandis que la France possède plus de $80 \%$ d'Électricité de France (EDF), d'AREVA et d'une série d'autres groupes de la branche énergétique, la

\footnotetext{
${ }^{9}$ Cf. Pawel Tokarski, « Economic Reforms in France. Bleak Macroeconomic Data Is Shaking Politics in Paris and Cementing France's Traditional Approach towards the Economic and Monetary Union » [en ligne], SWP Comments, $\mathrm{n}^{\circ} 39$, septembre 2014, p. 1-8. Disponible sur : http:/www.swp-berlin.org/fileadmin/contents/ products/comments/2014C39_tks.pdf [consulté le 06/05/2015].
} 
participation directe de l'État est anecdotique en Allemagne. L'ambivalence entre la nécessité de conserver des parts de marché d'un côté et de réformer le secteur pour augmenter la compétitivité des entreprises de l'autre reste donc un problème spécifiquement français. Ainsi, une contradiction subsiste entre la réduction de la part du nucléaire, en s'abstenant de construire de nouveaux réacteurs, et le rôle simultanément important du nucléaire pour EDF et AREVA à l'exportation. En Allemagne, on se demande en revanche de plus en plus jusqu'à quel point on peut pousser les électeurs à payer des subventions pour le développement de technologies, leur utilisation et la transformation du système énergétique. Sur un marché de l'électricité allemand fortement marqué par des structures concurrentielles, la capacité d'innovation n'est pas toujours récompensée, mais nécessite fréquemment un soutien de l'État. Pour les acteurs politiques, la nécessité de limiter ou d'ajuster ce soutien, comme dans le cas de la redevance sur les énergies renouvelables, s'est souvent avérée un combat rencontrant une farouche résistance. Même l'imprévisibilité des évolutions économiques, comme l'a montré l'exode de l'industrie solaire allemande, constitue un défi pour une politique économique qui mise avant tout sur des cadres précis et des subventions ciblées pour des branches et des technologies spécifiques.

Dans les deux pays, le secteur énergétique revêt une grande importance pour le marché du travail, même si les priorités sont différentes. Dans le cadre de l'Agenda 2010, la «modernisation écologique » était à l'ordre du jour d'un programme de réforme et de relance au début des années 2000 en Allemagne. À partir de 2005, la «politique industrielle écologique» de la grande coalition a misé sur des changements structurels pour stimuler la croissance. D'une manière similaire, le gouvernement français s'efforce aujourd'hui d'enclencher une dynamique comparable sous le vocable de «croissance verte». L'accent est également mis sur d'autres secteurs, comme la promotion de l'électromobilité dans la production automobile, ou celle du bâtiment dans le contexte des mesures d'efficacité énergétique.

Les deux projets coïncident aussi largement en matière d'intégration européenne et internationale. Lors des négociations sur le cadre de la politique énergétique et climatique de l'UE d'ici 2030, les deux gouvernements ont adressé des demandes similaires pour une réduction ambitieuse des émissions en Europe $^{10}$. Cette intervention commune avait déjà porté ses fruits en 2007-2008 dans le cadre des négociations du plan $2020^{11}$. Les deux pays soutiennent également la poursuite de la politique en matière d'énergies renouvelables et d'efficacité énergétique. Pour la France, l'organisation de la conférence internationale sur le climat (COP 21) fin 2015 à Paris a une résonance particulière en termes

\footnotetext{
${ }^{10}$ Cf. Severin Fischer, «Der neue EU-Rahmen für die Energie- und Klimapolitik bis 2030. Handlungsoptionen für die deutsche Energiewende-Politik » [en ligne], SWP-Aktuell, nº 73, décembre 2014, p. 1-8. Disponible sur : http://www.swp-berlin.org/fileadmin/contents/products/aktuell/2014A73_fis.pdf [consulté le 06/05/2015].

${ }^{11}$ Cf. Severin Fischer, «Die Neugestaltung der EU-Klimapolitik: Systemreform mit Vorbildcharakter? » [en ligne], Internationale Politik und Gesellschaft, $\mathrm{n}^{\circ}$ 2, 2009, p. 108-126. Disponible sur : http://library.fes.de/ pdf-files/ipg/ipg-2009-2/09_a_fischer_d.pdf [consulté le 06/05/2015].
} 
de politique intérieure. Le gouvernement français investit des ressources importantes pour la réussite de ces négociations. La mise en œuvre de la transition énergétique doit également être considérée dans ce contexte, où elle se veut un exemple sur la scène internationale.

CETTE RAPIDE COMPARAISON DES STRATÉGIES Nationales adoptées en Allemagne et en France pour la transformation du secteur énergétique montre que, malgré un grand nombre de points d'accord en matière d'orientation stratégique, de grandes divergences subsistent dans l'approche politique et la perception dans l'opinion publique des fonctions de l'État dans ce domaine. Le sujet de l'énergie atomique en particulier, mais aussi le rôle de la politique économique en lien avec le secteur de l'énergie restent marqués par des différences parfois importantes. S'y ajoute l'expérience déjà longue de l'Allemagne dans la restructuration de l'économie énergétique, alors que la France doit encore s'y atteler. À l'avenir, l'Allemagne et la France pourraient avoir à relever des défis comparables, la première avec le charbon et la seconde avec le nucléaire, qui jouent tous deux un rôle important dans la politique industrielle et de l'emploi de chacun des deux pays. Ce débat s'insérerait dans une coopération mûrie depuis des années entre les deux États, et dont l'organisation a subi elle aussi des transformations ces dernières décennies sur le plan du contenu et des acteurs concernés.

Traduction de Marie-Céline GEORG 



\section{Le tournant énergétique allemand ${ }^{1}$}

\section{Michel CRUCIANI}

Avec l'entrée dans le nouveau millénaire, la politique énergétique allemande a subi une inflexion majeure, concrétisant les attentes exprimées durant la décennie antérieure par les opposants à l'énergie nucléaire et les partisans des énergies renouvelables. Mis en œuvre par le gouvernement Schröder en 2000, le nouveau cadre réglementaire a produit progressivement ses résultats, très visibles à partir de 2005. Devenue chancelière à cette date, Angela Merkel a tenté une réorientation en 2010, en accordant un sursis aux centrales nucléaires, mais elle s'est vite ravisée et dès 2011 elle a fait du "tournant énergétique " un véritable étendard de la politique allemande. Les ajustements introduits en 2011 n'ont cependant pas permis de surmonter les difficultés apparues précédemment, et le dispositif législatif a connu un remaniement de grande ampleur en 2014, afin de contenir les coûts devenus exorbitants des deux filières privilégiées, éolienne et photovoltaïque.

Le présent article analyse les évolutions vécues outre-Rhin selon un déroulé chronologique. II se termine par une interrogation sur l'extension à l'échelle européenne des choix allemands... et des problèmes qu'ils engendrent. En effet, ayant surmonté la crise économique ouverte en 2008 mieux que la plupart des pays du continent, l'Allemagne a conquis une influence prépondérante dans les affaires européennes. En matière énergétique, elle pousse naturellement à une stratégie communautaire qui consolide ses propres choix, mais qui pourrait fragiliser les pays voisins.

\section{Rappel chronologique 2000-2011}

Après la catastrophe de Tchernobyl, l'opposition à l'énergie nucléaire en Allemagne trouve une nouvelle vigueur et les sources alternatives d'électricité connaissent un regain d'intérêt. En 1990, l'alliance formée par l'union chrétiennedémocrate et l'union chrétienne-sociale de Bavière (CDU-CSU) contrôlant le Bundestag fait adopter une loi introduisant la notion de tarif d'achat garanti pour l'électricité issue de sources renouvelables (Stromeinspeisegesetz du 7 décembre 1990). Les investissements décollent, notamment dans la production éolienne et la biomasse, mais l'effet de cette loi reste cependant contrarié à la fois par la réunification du pays, drainant les capitaux disponibles vers la mise à niveau des infrastructures, et par les incertitudes entourant la volonté de la Commission européenne de libéraliser le secteur de l'électricité.

En 1998, une alliance entre le parti social-démocrate (SPD) et le parti écologiste, la coalition rouge-verte, conquiert le pouvoir fédéral. L'accord de gouvernement inclut la «modernisation écologique » du secteur de l'énergie. Encouragée par le chancelier Gerhard Schröder, elle est menée en grande partie par le ministre de l'Environnement, Jürgen Trittin, issu du parti écologiste (Bündnis 90/Die

\footnotetext{
${ }^{1}$ Article rédigé en juin 2015, NdE.
} 
Grünen), épaulé par des élus du SPD hostiles à l'énergie nucléaire, tels que le député Hermann Scheer. Cette politique se traduit par deux actes majeurs :

- l'adoption de la loi sur les énergies renouvelables, dite loi EEG (Erneuerbare-Energien-Gesetz du 29 mars 2000). Dans son chapitre concernant la production d'électricité, cette loi étend le dispositif antérieur, confiné auparavant aux petites installations. Le tarif d'achat est garanti sur 20 ans, à un prix connu à l'avance et le surcoût au regard du prix du marché est répercuté sur le consommateur final. En 2004, une retouche à ce dispositif permet un ajustement du tarif aux coûts des équipements ;

- la signature d'une convention avec les exploitants de centrales nucléaires, le 14 juin 2001. Elle fixe un plafond à la production cumulée des 19 réacteurs en service ( $2620 \mathrm{TWh}$ ) et une durée moyenne d'exploitation (32 ans), en laissant toutefois la possibilité d'affecter cette production et la durée de vie selon un barème spécifique à chaque centrale. Le dernier réacteur nucléaire serait arrêté en 2021. Cette convention est transcrite dans la loi le 22 avril 2002, sous le titre «Sortie du nucléaire » (Atomausstieg), par un amendement à la loi autorisant le pays à recourir à l'usage pacifique de l'atome (Atomgesetz du 23 décembre 1959).

Ce nouveau cadre législatif conduit d'une part à un développement remarquable de l'électricité d'origine renouvelable durant toute la décennie 2000-2010, d'autre part à la fermeture de deux réacteurs nucléaires (Stade et Obrigheim).

Toutefois, les grandes compagnies électriques allemandes ${ }^{2}$ ne semblent pas entrevoir les risques de recomposition sectorielle induits par la loi EEG et cette myopie s'explique peut-être par l'attitude bienveillante des gouvernements successifs à leur égard. Ces derniers autorisent ainsi en 2000 le rapprochement entre les conglomérats VEBA et VIAG, maisons mères des groupes PreussenElektra et Bayernwerk, dont la fusion donne naissance au géant de l'énergie E.ON. Celui-ci obtient à son tour en 2003 l'autorisation d'absorber le groupe Ruhrgas afin d'élargir encore sa palette d'activités. Dans les deux cas, le gouvernement allemand défend la consolidation auprès de la Commission européenne, pourtant hostile aux fusions : elle interdit à la même époque le mariage entre les entreprises françaises Legrand et Schneider.

Sur d'autres terrains également, la chancellerie réussit à préserver des spécificités allemandes très favorables aux compagnies électriques en place. Ainsi, alors que l'ouverture du marché aux producteurs électriques des pays voisins est rendue obligatoire depuis la directive européenne de 1996, les importations restent verrouillées jusqu'en 2005 par l'obligation faite aux concurrents étrangers de négocier le passage du courant avec les gestionnaires allemands des réseaux, qui ne font rien pour faciliter les accords... L'électricien français EDF comprend que pour exporter du courant outre-Rhin, il faut devenir allemand et prend donc une

\footnotetext{
${ }^{2}$ Cette expression désigne ici les groupes EnBW, E.ON, RWE et Vattenfall qui assuraient l'essentiel de la
} production d'électricité en Allemagne au début des années 2000. 
participation au sein du groupe EnBW. Les compagnies reçoivent aussi un traitement de faveur lors de la mise en œuvre du système européen d'échange de quotas de $\mathrm{CO}_{2}\left(\mathrm{au} 1^{\mathrm{er}}\right.$ janvier 2005) : leur dotation est calculée selon des modalités extrêmement avantageuses. Enfin, l'Allemagne bénéficie d'une dérogation aux contraintes européennes, évitant aux entreprises locales (les Stadtwerke) de scinder leurs activités; la directive de 2009 sur le marché intérieur de l'électricité confirme que cette clause, destinée à placer tous les fournisseurs sur un pied d'égalité, ne s'applique pas à près de 800 acteurs locaux en territoire germanique.

Fortes de ce soutien gouvernemental sur leur cœur de métier, les grandes entreprises allemandes continuent de croire en l'avenir de l'énergie nucléaire. Le groupe Siemens approuve en 2001 un partenariat avec le groupe AREVA pour développer cette technologie; il dénonce cet accord en 2009, mais en signe aussitôt un autre avec le groupe d'État russe Rosatom, un spécialiste du domaine nucléaire à taille mondiale. En 2009 encore, les groupes E.ON et RWE s'allient au sein d'une filiale commune, Horizon Nuclear Power, créée pour participer à la relance de cette énergie en Grande-Bretagne.

Le relatif désintérêt des électriciens «historiques » pour les énergies renouvelables transparaît dans les chiffres : en 2010, ils ne détenaient que 4 GW des $53 \mathrm{GW}$ de sources renouvelables alors en service, soit à peine $7 \%$. Leur choix s'explique aussi par les changements de majorité gouvernementale. Après un premier basculement en 2005, une coalition entre le parti chrétiendémocrate (CDU) et le parti libéral (FDP) arrive au pouvoir en 2009, en ayant promis de réviser la décision de sortie du nucléaire.

Cette volonté est confortée par l'échec de la conférence de Copenhague, fin 2009. Entre 2001 et 2009, l'Union européenne (UE) emboîte le pas à l'Allemagne et promeut le développement des énergies renouvelables, avec deux directives imposant des mesures en leur faveur (électricité en 2001 et biocarburants en 2003). L'UE accorde aussi une grande importance à la lutte contre les émissions de gaz à effet de serre, mettant en place un système d'échange de quotas d'émission (SEQE) dès 2005. Les alertes contre les conséquences $\mathrm{du}$ changement climatique se multiplient. Le rapport Stern et le film d'Al Gore en 2006, puis le quatrième rapport du GIEC en 2007, rencontrent une large audience ${ }^{3}$. Les dirigeants européens croient alors en la perspective d'un accord mondial à Copenhague, lors de la quinzième conférence des parties à la convention de Rio ${ }^{4}$. Pour se présenter à cette conférence en position exemplaire, les chefs d'État et de gouvernement de l'UE, soutenus par le Parlement européen, adoptent un paquet énergie-climat comportant des objectifs contraignants pour chaque État membre de l'Union. Mais le refus de la Chine et des

\footnotetext{
${ }^{3}$ Sir Nicholas Stern, Review on the Economics of Climate Change, Cambridge University Press, Cambridge, 2006.

Une vérité qui dérange, film réalisé par Davis Guggenheim à partir des publications de l'ancien vice-président des USA.

GIEC : Groupe intergouvernemental d'experts sur l'évolution du climat (IPCC en anglais).

${ }^{4}$ Convention-cadre des Nations unies sur les changements climatiques (CCNUCC), signée en 1992.
} 
États-Unis de s'engager sur un texte multilatéral ruine les espoirs de bâtir un cadre international.

Après l'échec de Copenhague, et au terme d'une longue concertation, la chancelière Angela Merkel présente un plan d'ensemble, la «trame énergétique » (Energiekonzept 2010, ou EK 2010). Elle définit une orientation énergétique extrêmement ambitieuse jusqu'à l'horizon 2050, comparable par sa résonnance aux lois «Grenelle de l'environnement» de 2009 et 2010 en France. Le texte est approuvé par le Bundestag le 29 octobre 2010. Il prévoit la prolongation de la durée de vie des centrales nucléaires de 8 à 14 ans, selon leur âge, au-delà de 2021. En échange, les exploitants s'engagent sur un programme d'investissements accrus pour renforcer leur sûreté. Ils acceptent en outre de payer d'une part une taxe sur le combustible nucléaire, dont le produit est versé au budget de l'État (en ce temps-là déficitaire...), d'autre part une contribution au fonds «Énergie et Climat », qui servira notamment à stimuler le développement des énergies renouvelables. L'énergie nucléaire est alors décrite comme une énergie de transition, dont la préservation reste nécessaire jusqu'à l'arrivée à maturité des énergies renouvelables, qui domineront le paysage énergétique allemand en 2050.

Les dispositions favorables à l'énergie nucléaire se révèlent aussitôt impopulaires. Soucieux de s'en désolidariser, le gouvernement du Land de BadeWurtemberg, appartenant pourtant à la majorité fédérale menée par la CDU, demande en toute hâte à EDF de céder sa participation au sein du groupe EnBW. Les élections régionales de mars 2011 s'annoncent en effet périlleuses pour l'équipe sortante; elle tient à afficher son indépendance à l'égard d'un opérateur électrique identifié comme pronucléaire. Un accord, avantageux pour EDF, est signé le 6 décembre 2010.

L'accident de Fukushima survient le 11 mars 2011. La chancelière Angela Merkel comprend immédiatement le parti qu'elle peut tirer des circonstances pour rétablir son image ; elle agit très rapidement :

- dès le 14 mars, la chancelière annonce un moratoire de trois mois, mettant immédiatement à l'arrêt les huit réacteurs nucléaires les plus anciens ;

- le 17 mars, elle demande à la Commission de sûreté des réacteurs $\left(\mathrm{RSK}^{5}\right)$ de procéder à un examen des centrales allemandes à la lumière des évènements survenus au Japon ;

- le 22 mars, une commission éthique est installée par la chancelière. Sa mission consiste à évaluer les risques présentés par l'énergie nucléaire et à examiner la faisabilité d'une sortie de cette énergie pour «entrer dans le siècle des énergies renouvelables ». La commission est coprésidée par Klaus Töpfer, ancien ministre (CDU) du chancelier Helmut Kohl, et Matthias Kleiner, alors président de l'Agence allemande de financement de la recherche (DFG) ;

\footnotetext{
${ }^{5}$ RSK (Reaktor Sicherheitskommission), autorité fédérale de sûreté, chargée depuis 1958 du contrôle de la sûreté des installations nucléaires et de la protection contre les rayonnements ionisants.
} 
- le 15 mai, la RSK remet son rapport, estimant que le parc nucléaire allemand ne présente pas de risque générique, mais préconisant des tests de sûreté spécifiques sur chaque site. Elle émet cependant un doute sur la sûreté des centrales les plus anciennes en cas de chute d'un avion gros porteur ;

- le 28 mai, la commission éthique remet son rapport au gouvernement, avant de le rendre public deux jours plus tard. Selon les conclusions du rapport, l'exploitation de l'énergie nucléaire fait courir des risques à des populations qui ne sont associées ni aux décisions ni au contrôle de leur mise en œuvre : il s'agit des peuples vivant en dehors du pays d'établissement des centrales ou des générations futures appelées à vivre dans ce pays. En ce sens, exploiter l'énergie nucléaire ne remplit pas toutes les conditions éthiques. Le rapport estime par ailleurs que l'Allemagne pourrait obtenir une sécurité d'approvisionnement équivalente à son niveau actuel en arrêtant progressivement les centrales nucléaires et en développant les énergies renouvelables. Il préconise cette voie ;

- le 30 mai, le ministre de l'Environnement, Norbert Röttgen, annonce que les huit réacteurs à l'arrêt ne redémarreront pas, et que les neuf autres seront fermés d'ici 2022 ;

- le 6 juin, un projet de loi en ce sens est adopté lors d'un conseil des ministres extraordinaire ; le texte est soumis au Bundestag et voté à une majorité écrasante le 30 juin 2011, puis approuvé par le Bundesrat le 8 juillet 2011.

Dans les semaines qui suivent, les députés adoptent divers autres textes remodelant le cadre légal du secteur de l'énergie allemand, l'ensemble marquant un véritable «tournant énergétique » (Energiewende). Plusieurs des textes approuvés durant cette période comportent des clauses permettant leur mise à jour ultérieure par voie d'ordonnance, sans consultation du Bundesrat. Un point essentiel mérite attention: les objectifs volontaristes du plan antérieur (Energiekonzept 2010) sont conservés, aussi bien la réduction des émissions de gaz à effet de serre que la réduction des consommations d'énergie et le développement des énergies renouvelables. Or l'atteinte de ces objectifs devait être facilitée par le maintien d'une production nucléaire offrant au pays une électricité peu onéreuse, non émettrice de $\mathrm{CO}_{2}$ et pourvoyeuse de fonds pour la politique énergétique ; désormais il faut réussir avec des apports considérablement réduits de la part du secteur nucléaire.

\section{Évolution de 2011 à 2015}

Les objectifs confirmés en 2011 contiennent quatre repères majeurs pour 2020 :

- une réduction des consommations d'énergie de $20 \%$ (par rapport à leur niveau de 2008); 
- une réduction des émissions de $\mathrm{CO}_{2}$ de $40 \%$ (par rapport à leur niveau de 1990);

- une part de $18 \%$ d'énergies renouvelables dans la consommation totale ;

- une part de $35 \%$ de sources renouvelables dans la consommation d'électricité.

Cependant, dans le «paquet» législatif traité en 2011, l'essentiel des dispositions conforte le soutien aux sources renouvelables d'électricité, qui devient de fait l'objectif phare de l'Energiewende. La réduction de la consommation d'énergie continue à reposer sur le dispositif antérieur, certes solide grâce aux aides sur fonds publics, mais sans mesure novatrice. Aucun tournant significatif non plus pour les énergies autres que l'électricité. Pour la réduction des émissions de $\mathrm{CO}_{2}$, le gouvernement comptait sur un projet de loi autorisant le captage et le stockage du $\mathrm{CO}_{2}$. Il visait à faciliter le lancement de démonstrateurs à l'échelle industrielle, en autorisant la construction de réseaux de $\mathrm{CO}_{2}$ jusqu'aux sites de stockage souterrain les plus appropriés, fussent-ils situés hors de la région d'origine. Or ce texte est rejeté par le Bundesrat le 23 septembre 2011. En effet, les Länder situés sur les structures géologiques les plus favorables craignent de devenir les « dépotoirs » du $\mathrm{CO}_{2}$ produit dans tout le pays, alors que le stockage souterrain suscite encore méfiance, voire opposition dans une partie de la population. Une hostilité comparable frappe l'exploitation du gaz de schiste : la fracturation hydraulique demeure bannie de facto.

\section{Graphique 1 : Production d'électricité d'origine renouvelable en Allemagne}

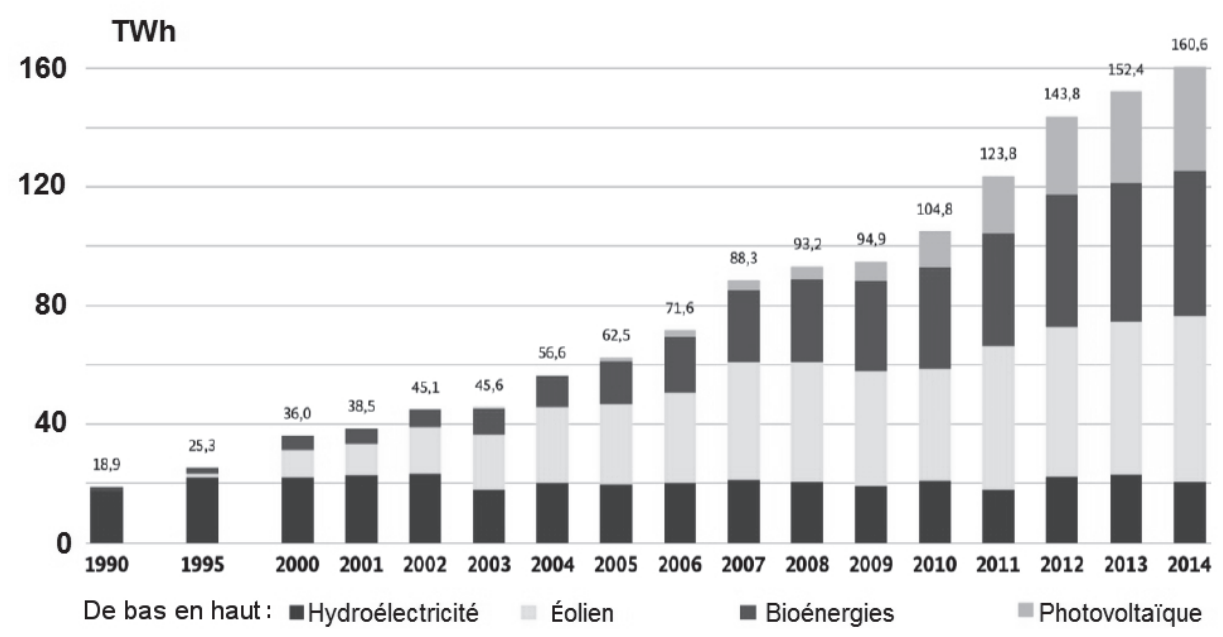

Source : Bundesministerium für Wirtschaft und Energie (BMWi), Erneuerbare Energien im Jahr 2014, Berlin, 27 février 2015.

La production d'électricité d'origine renouvelable avait déjà connu une accélération à partir de 2007, grâce à la baisse du prix des composants et à la diffusion du 
savoir-faire dans les branches professionnelles concernées. Confortée par la priorité politique qui lui est accordée en 2011, l'électricité d'origine renouvelable reçoit une nouvelle impulsion et progresse à un rythme exceptionnel ; elle représente déjà $27 \%$ de l'électricité consommée en 2014. Le graphique 1 ci-dessus rend compte de ce double élan ; il illustre la place considérable des sources éolienne et photovoltaïque, malgré les inconvénients liés à leur caractère intermittent.

Cependant, cette croissance spectaculaire comporte aussi des zones d'ombre.

En premier lieu, ses bénéfices industriels restent inégaux. Le secteur éolien garde un taux d'activité satisfaisant, mais les entreprises du secteur photovoltaïque ont fortement reculé. En effet, dans l'espoir de canaliser le développement de cette énergie, le gouvernement a réduit la rémunération du courant qu'elle produit. Ce geste a abouti à un résultat imprévu : les panneaux produits en Allemagne ont été évincés par les modules beaucoup moins chers fabriqués en Asie (principalement de Chine), et la mise en place de filières d'importation bon marché a donné un coup de fouet à l'énergie photovoltaïque... tout en ruinant les producteurs de panneaux allemands, qui figuraient auparavant parmi les leaders mondiaux. En 2008, environ 43 \% des capacités photovoltaïques en service sur la planète étaient installées en Allemagne, qui détenait $17 \%$ du marché mondial. En 2013, l'Allemagne possédait $25 \%$ des capacités mondiales, mais ses usines n'approvisionnaient plus que $2 \%$ du marché.

En second lieu, l'électricité issue de sources renouvelables se substitue à celle venant des réacteurs nucléaires, sans entamer les volumes produits par les centrales alimentées en combustibles fossiles. Parmi ces derniers, les plus polluants (lignite et houille) ont pris l'avantage sur le moins nocif (gaz naturel), en raison de prix de marché plus avantageux en 2012 et 2013, de sorte que les émissions de $\mathrm{CO}_{2}$ de l'Allemagne ont augmenté de $3 \%$ entre 2011 et 2013. Le mécanisme des quotas de $\mathrm{CO}_{2}$ instauré à l'échelle européenne n'a pas joué son rôle de frein, car le gouvernement avait distribué généreusement les quotas lorsqu'ils étaient gratuits et la crise économique a réduit les besoins dans la plupart des pays entourant l'Allemagne, faisant chuter le prix de la tonne de $\mathrm{CO}_{2}$ à un niveau sans conséquence pour les opérateurs. On notera incidemment l'attitude ambiguë du gouvernement allemand, qui n'a pas montré d'ardeur particulière à réformer le $\mathrm{SEQE}$ et a ferraillé contre le projet de durcir les normes européennes relatives au $\mathrm{CO}_{2}$ émis par les voitures...

En dernier lieu, le succès des sources renouvelables d'électricité repose sur la propension à payer des consommateurs allemands, ceux-ci assumant le surcoût de ces sources par rapport aux centrales conventionnelles. Ce surcoût a pris des proportions considérables; en 2014 il atteignait 23,5 milliards d'euros. Cette somme n'est pas ventilée équitablement entre tous les clients : la grande industrie bénéficie d'exemptions. L'essentiel du surcoût est répercuté sur les consommateurs domestiques sous forme d'une surcharge (EEG-Umlage) qui représente en 2014 et 2015 environ $21 \%$ du prix du kWh pour les ménages, lequel reste le deuxième plus cher d'Europe (le premier étant celui du Danemark). 
Dès 2013, l'augmentation incontrôlée des factures prend une tournure politique. Les élections fédérales du 22 septembre 2013 voient l'effacement du parti libéral et l'arrivée du parti social-démocrate (SPD) au sein du gouvernement ; l'accord négocié au sein de la nouvelle coalition comprend en bonne place une refonte de la loi sur les énergies renouvelables. Le dossier est confié au nouveau ministre en charge de l'Économie et de l'Énergie Sigmar Gabriel (SPD), qui se rend immédiatement à Bruxelles pour obtenir l'assurance que le régime dérogatoire accordé à la grande industrie ne sera pas remis en cause. Il est entendu : les lignes directrices présentées par la Commission européenne le 9 avril $2014^{6}$ autorisent ce type d'exemptions, selon des modalités que le projet de loi allemand reprend scrupuleusement. Le texte est voté par le Bundestag le 27 juin et approuvé par le Bundesrat le 11 juillet 2014.

La loi, dite «EEG $2.0 »$, permet un contrôle strict de la rémunération des producteurs d'électricité d'origine renouvelable en l'enserrant dans un «corridor » qui fluctue en fonction des résultats. Si ceux-ci dépassent les objectifs annuels, la rémunération est immédiatement amputée ; elle est majorée dans le cas inverse. Ces objectifs eux-mêmes favorisent la source la moins dispendieuse : l'énergie éolienne à terre, avec une trajectoire de $2500 \mathrm{MW} / \mathrm{an}$. L'énergie photovoltaïque apparaît bridée, avec le même objectif (2 $500 \mathrm{MW} / \mathrm{an}$ ) alors que les années antérieures avaient vu des mises en service plus conséquentes: 3300 MW en 2013 et 7600 MW en 2012 ! L'énergie éolienne en mer reçoit un objectif inférieur : 6500 MW d'ici 2020 et l'électricité issue de biomasse (solide ou biogaz) est quasiment abandonnée, avec $100 \mathrm{MW}$ par an seulement. Pour cette dernière source, le gouvernement suit l'opinion publique, choquée par les cultures intensives de maïs destinées à la production de biogaz, tandis que le pays importe du maïs alimentaire.

Au passage, la nouvelle loi restreint l'avantage dont bénéficiaient auparavant les autoconsommateurs, c'est-à-dire les propriétaires d'installations (photovoltaïques principalement) destinées à leur propre alimentation. Ce courant électrique était totalement exonéré de la surcharge EEG ; elle s'appliquera désormais partiellement (à hauteur de $40 \%$ ) à chaque $\mathrm{kWh}$ autoconsommé. Cette mesure ne constitue qu'une correction mineure de l'un des effets redistributifs engendrés par la loi initiale, depuis l'an 2000. Elle atténue l'inégalité entre le consommateur ordinaire et l'autoconsommateur, mais ne l'élimine pas.

La loi de 2014 ne réduit en rien une autre forme d'inégalité, celle qui favorise les épargnants, prêts à investir dans des installations d'énergie renouvelable, en général très rentables grâce au principe du tarif d'achat garanti, principe maintenu par le nouveau texte malgré des modalités différentes. Le consommateur aux ressources insuffisantes pour épargner continuera donc à payer son électricité à un prix élevé afin de rémunérer son voisin qui a pu placer des liquidités dans les sources renouvelables. La loi maintient aussi, comme in-

${ }^{6}$ Lignes directrices concernant les aides d'État à la protection de l'environnement et à l'énergie pour la période 2014-2020, document 2014/C 200/01, publié au Journal officiel de l'Union européenne le 28 juin 2014. 
diqué ci-dessus, une autre forme de transfert financier, des consommateurs domestiques vers la grande industrie. Les exonérations relatives à la surcharge EEG dont bénéficie cette dernière peuvent être estimées à 4,8 milliards d'euros pour l'année 2014, somme qui s'apparente à une subvention indirecte de la part des ménages, car ceux-ci acquittent le montant non assumé par l'industrie. Signalons en outre que les gros consommateurs d'électricité ne paient pas l'intégralité des frais de réseau qui leur incombent : un abattement leur est consenti, chiffré à 630 millions d'euros pour l'année $2014^{7}$.

Par ailleurs, la loi EEG 2.0 n'apporte aucune réponse à trois dossiers sensibles politiquement :

- les difficultés financières des producteurs conventionnels. Rémunérée en dehors des lois du marché, l'électricité issue de sources renouvelables tire vers le bas le prix appliqué à l'ensemble des productions. Les revenus des entreprises possédant les centrales classiques chutent, érodant leur valeur boursière, poussant à leur endettement et restreignant les possibilités d'investissement. Or ces centrales demeurent indispensables à la sécurité d'approvisionnement : elles assurent encore l'essentiel de la production allemande et fonctionnent en l'absence de vent et de soleil ;

- les émissions de $\mathrm{CO}_{2}$. En 2011, le gouvernement espérait que les détenteurs des anciennes centrales au charbon les remplaceraient progressivement par des unités modernes, dotées d'un meilleur rendement (notamment lorsqu'elles mettent en œuvre la technologie de la cogénération), et recourant davantage au gaz, nettement moins émetteur de $\mathrm{CO}_{2}$ par $\mathrm{kWh}$ produit. Cependant, les difficultés financières des entreprises exploitant ces centrales, le prix avantageux du charbon et les obstacles juridiques entravant la construction de centrales neuves ont compromis les perspectives tracées par l'Energiewende ;

- les extensions de réseau. Dans leur majorité, les fermes éoliennes sont érigées dans les régions situées au nord du pays, les mieux ventées, et ces régions recevront bientôt les grands parcs éoliens en mer prévus d'ici 2030. Or les principaux lieux de consommation industrielle restent implantés au sud, et les liaisons électriques à haute tension entre les deux zones ne répondent plus aux besoins. Bien que les lois adoptées durant l'été 2011 comportent des clauses visant à accélérer la construction des lignes, les oppositions à ces ouvrages demeurent vives

\footnotetext{
${ }^{7}$ Bundesverband der Energie- und Wasserwirtschaft e.V. (BDEW), BDEW Strompreisanalyse Juni 2014 Haushalte und Industrie [en ligne], Berlin, 2014. Disponible sur: https://www.bdew.de/internet.nsf/id/ 20140702-pi-steuern-und-abgaben-am-strompreis-steigen-weiter-de/\$file/140702\%20BDEW\%20Strompreis analyse\%202014\%20Chartsatz.pdf [consulté le 29/07/2014]. Le montant de l'abattement sur les dépenses de réseau figure en page 24 ; le montant des exonérations pour la surcharge EEG résulte d'un calcul de l'auteur à partir des données de la page 26.
} 
de la part des riverains, de sorte que les constructions ont pris un retard considérable.

Ces trois dossiers concernent aussi les pays entourant l'Allemagne. Sous l'impulsion de la Commission européenne, les pays situés à l'ouest du continent ont réalisé le «couplage » des marchés de l'électricité ; sous l'influence des excédents allemands, les prix de gros sont entraînés partout à la baisse, ce qui fragilise toutes les grandes entreprises de la zone. Le SEQE établit un prix du $\mathrm{CO}_{2}$ à l'échelle européenne ; toute solution que l'Allemagne mettra en œuvre pour juguler ses émissions comportera des conséquences pour l'ensemble de l'Union européenne. Enfin, en raison de l'insuffisante capacité des réseaux intérieurs allemands, une partie du courant produit au nord du pays transite par les pays limitrophes avant de revenir au sud, saturant à certaines périodes les réseaux des Pays-Bas, de la Belgique et de la France d'un côté, et de la Pologne, de la République tchèque et de l'Autriche de l'autre côté.

Cependant, malgré les difficultés qu'ils observent en Allemagne et malgré les conséquences négatives de sa politique qu'ils subissent sur leur sol, une majorité de pays européens semblent prêts à suivre la piste ouverte outre-Rhin. Les Conseils européens de juin et octobre 2014 ont en effet bien accueilli la proposition de la Commission de viser un objectif de $27 \%$ de sources renouvelables pour l'ensemble des énergies en 2030 , ce qui équivaut à $45 \%$ pour l'électricité.

Or l'Allemagne a pu atteindre $27 \%$ d'électricité d'origine renouvelable grâce à des spécificités que peu de pays partagent. Elle dispose d'une structure fédérale, propice à une gestion décentralisée, bien adaptée à ce type de projets à dominante locale, et elle s'appuie sur un tissu de banques régionales détenant une réelle expertise dans le secteur de l'énergie. L'Allemagne sert de refuge aux capitaux depuis la crise de 2008 ; sa banque publique KfW a ainsi pu drainer les liquidités des marchés mondiaux et irriguer les circuits de financement à des taux très avantageux. Les coopératives locales restent dynamiques et permettent une implication des citoyens dans les projets ; il existe par ailleurs un consensus unique sur l'acceptation de factures élevées par le consommateur domestique et sur le soutien à l'industrie grâce à des exemptions considérables. Enfin le pays possède un parc de centrales conventionnelles complémentaires des installations renouvelables et il peut exporter ses excédents de production vers les pays proches ; ce ne sera évidemment plus possible si ces derniers deviennent aussi exportateurs au même moment. Tant que l'on n'aura pas mis au point des solutions de stockage massif de l'électricité, de l'ordre de plusieurs mois de consommation, il faudra pourtant trouver un débouché aux volumes produits durant les saisons de fort vent et grand soleil.

L'AlLEMAGNE A AMORCÉ DEPUIS LE DÉBUT DU MILLÉNAIRE une transition énergétique majeure ; cependant, des conditions exceptionnellement favorables ne l'ont pas empêchée de rencontrer des difficultés, qui ne sont pas encore surmontées. On peut en déduire qu'une pleine réussite suppose une progression au 
même rythme de tous les facteurs : construction des installations renouvelables, mais aussi reconversion du parc existant, adaptation des modalités de consommation, traitement des effets redistributifs... En allant trop vite, on ne tire pas tout le bénéfice des acquis de l'expérience, notamment pour la gestion des réseaux. Un tel constat devrait inciter les autres pays européens à la prudence, continuant certes à encourager les énergies renouvelables, mais sans s'imposer un objectif dont l'atteinte exigera des sacrifices susceptibles de susciter des mouvements de rejet dans la population (augmentation des factures, construction de lignes à haute tension, etc.), d'autant plus marqués que sa situation économique est dégradée.

De son côté, l'Allemagne ménagerait l'avenir en associant davantage ses voisins à la résolution des problèmes que pose l'injection massive de courant issu de sources intermittentes dans le système électrique européen. Elle a décidé sans concertation avec les pays limitrophes de fermer son parc nucléaire, de solliciter leurs réseaux pour ses besoins propres, d'affaiblir leurs entreprises nationales par l'exportation de ses excédents. Son poids politique en Europe lui permettrait d'aménager encore son cadre national sans trop se préoccuper des conséquences pour les voisins, par exemple pour réduire les émissions de $\mathrm{CO}_{2}$ de ses vieilles centrales par des dispositions internes plutôt qu'une réforme du SEQE ou pour garantir sa sécurité d'alimentation par des mécanismes locaux inaccessibles aux entreprises étrangères plutôt que par un modèle ouvert à tous. Ce faisant, l'Allemagne prendrait alors le risque que les autres États agissent aussi de manière non coopérative et vident de sa substance l'Europe de l'énergie. 



\section{Thèses essentielles sur les phases de transformation du système énergétique : réflexions sur le tournant énergétique en Allemagne ${ }^{1}$}

\section{Manfred FISCHEDICK}

En adoptant son projet énergétique (Energiekonzept) mi-20112, le gouvernement allemand a formulé des objectifs ambitieux pour la transformation durable du système énergétique : sortie de l'énergie nucléaire pour la production d'électricité d'ici 2022, réduction des émissions de gaz à effet de serre d'au moins 80 \% d'ici le milieu du siècle par rapport à 1990, augmentation de la part des énergies renouvelables dans la production d'électricité pour atteindre $80 \%$ et division par deux de la consommation d'énergie primaire par rapport au niveau de 2008 dans le même délai, pour ne citer que quelques exemples. Pour atteindre ces objectifs, le gouvernement fédéral mise principalement sur l'exploitation du potentiel des énergies renouvelables (en particulier éolienne et solaire) ainsi que sur l'amélioration systématique de l'efficacité énergétique. Le chemin esquissé par le gouvernement ne constitue ni plus ni moins qu'un changement total, pour passer d'un système actuel largement dominé par les sources d'énergie fossiles à un système reposant nettement sur les énergies renouvelables. C'est à juste titre que ce processus est qualifié de " tournant énergétique » (Energiewende).

Outre-Rhin, une longue culture de débat sur la transition énergétique

Le débat sur les alternatives en matière d'approvisionnement énergétique n'est pas récent en Allemagne ; il s'est ouvert bien avant la publication de l'Energiekonzept mi-2011. Dès la fin des années 1970, à l'époque très marquées par les manifestations concernant l'extension de l'énergie nucléaire, on a vu les premiers livres portant le titre «Energiewende ». Dans ces livres, la transition énergétique était alors abordée sous l'angle de «[...] la faisabilité du nécessaire » ou de «[...] la croissance et la prospérité sans pétrole ni uranium». Aujourd'hui, certains des objectifs formulés à cette occasion se retrouvent sous une forme adaptée dans les décisions gouvernementales formelles.

Même si la longue culture de débat sur l'approvisionnement énergétique du futur a certainement contribué à forger un socle substantiel de connaissances, non seulement sur la nécessité de changer, mais aussi et surtout sur les possibilités de changement, il reste d'énormes défis à relever en termes de réalisation pratique du tournant énergétique. On ne peut que constater que, du point de vue

\footnotetext{
${ }^{1}$ Article rédigé en juin 2015, NdE.

${ }^{2}$ Le projet énergétique de 2011 reprend les objectifs de celui adopté un an plus tôt par le gouvernement allemand. Il intègre en outre la sortie progressive du nucléaire, conséquence des accidents survenus en mars 2011 sur les réacteurs de la centrale de Fukushima au Japon.
} 
actuel, la mise en œuvre de l'Energiewende constitue un processus de transformation ambitieux et en même temps hautement complexe, pour lequel on ne dispose à ce jour d'aucun modèle.

\section{Développer les énergies renouvelables et accroître l'efficacité énergétique}

Dans ce contexte, les thèses essentielles ci-après veulent contribuer à mieux faire comprendre ces défis et à définir des pistes pour les actions futures.

En observant d'autres processus de transformation, on peut tirer des leçons utiles pour l'organisation du tournant énergétique. C'est souvent à la suite de crises ou de situations de pénurie que de profondes mutations se sont produites, par exemple lorsque les structures existantes ont atteint leurs limites et que les modes de comportement sont devenus impossibles à maintenir ou que des domaines d'activités établis se sont détériorés. Ces mutations ont pu s'imposer lorsque les possibilités technologiques étaient suffisantes et qu'il était possible de les intégrer dans des contextes institutionnels, sociaux et culturels adéquats. Les transformations sont encouragées par les idées innovantes qui ont fait la démonstration de leur succès et constituent une source de modification des structures existantes (par exemple les structures de marché). Le débat social et la participation à différents niveaux jouent un rôle d'accélérateur pour la mise en œuvre de processus complexes de transformation, au même titre que l'orientation vers une stratégie commune. Un parfait exemple en est l'appel au « ciel bleu au-dessus de la Ruhr» lancé il y a plus de 50 ans par Willy Brandt, alors candidat au poste de chancelier.

Le tournant énergétique doit être compris comme une mission globale de réorganisation du système dans son ensemble, en tenant compte des synergies et des rétroactions négatives entre les différents secteurs. Dans ce contexte, le tournant énergétique est bien plus qu'un tournant électrique, même si la production d'électricité a un rôle de premier plan dans la décarbonation du système énergétique. Ce tournant ne se résume pas non plus à une affaire de mise à disposition et de distribution durables d'énergie. Pour qu'il soit réussi, il faut non seulement développer les énergies renouvelables et leur intégration dans le système, mais aussi et surtout augmenter sérieusement l'efficacité énergétique dans tous les secteurs, depuis le rendement des centrales électriques jusqu'à l'exploitation des nombreux potentiels d'efficacité énergétique - parfois très intéressants économiquement - dans les ménages, les transports, le commerce et l'industrie.

La décarbonation du système énergétique peut être divisée en quatre phases, les frontières entre les secteurs devenant toujours plus floues au fil du temps, ce qui nécessite de plus en plus des solutions globales. La première phase est celle de la prise de conscience, du développement et de la mise sur le marché de nouvelles technologies, de la construction de nouvelles structures et de l'exploitation des effets de dégressivité des coûts. En ce qui concerne la production d'élec- 
tricité à partir d'énergies renouvelables, avec une part de marché de $25 \%$ et plus, nous avons déjà dépassé cette phase. La deuxième phase, peut-être la plus décisive, correspond à une part d'énergies renouvelables dans la production d'électricité comprise entre 25 et $60 \%$. Elle est placée sous le signe de l'intégration des énergies renouvelables et de la gestion technique et économique intelligente des interactions entre les différentes technologies. Dans la troisième phase, où la part de marché des énergies renouvelables se situe entre 60 et $100 \%$, c'est le stockage (à long terme) qui prime, avec la poursuite de l'intégration européenne et la construction de structures transsectorielles. Le surplus de courant provenant d'énergies renouvelables est de plus en plus souvent converti en hydrogène, en gaz naturel synthétique ou en carburants (Power-to- $X$ ), qui sont principalement utilisés comme source d'énergie finale dans le domaine des transports et de l'industrie, c'est-à-dire là où les possibilités d'utilisation directe de l'électricité sont limitées. La quatrième phase consiste à poursuivre l'exclusion des énergies fossiles du secteur des énergies finales. L'électricité provenant d'énergies renouvelables et ses produits de conversion représentent alors l'élément central de la décarbonation du système énergétique dans son ensemble. À ce stade, la production d'électricité à partir d'énergies renouvelables dépasse nettement la demande (classique) en courant des secteurs consommateurs.

\section{Graphique 1 : Schéma des phases d'évolution du système énergétique}

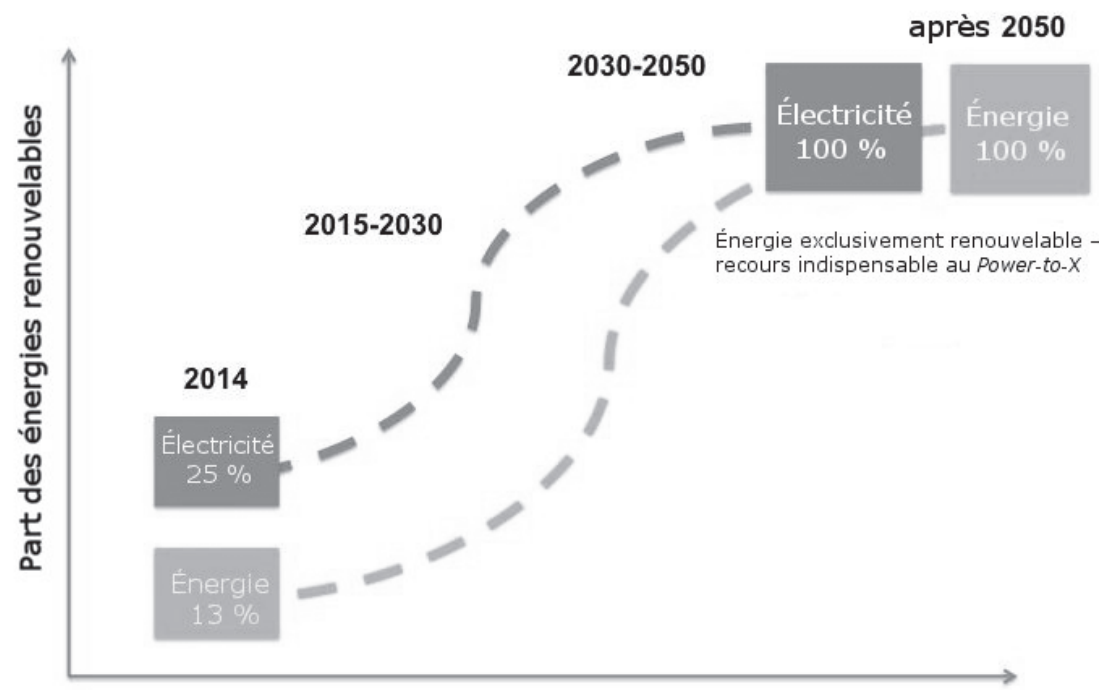

Source : présentation de l'auteur.

\section{Un enjeu à la fois politique, économique, technologique et social}

En fin de compte, la mise en œuvre du tournant énergétique ne va pas de soi. C'est un défi à la fois technologique, économique et social, qui exige un 
changement des mentalités à différents niveaux. Il est par exemple nécessaire de redéfinir totalement le modèle actuel du marché de l'énergie et de l'électricité. Les énergies renouvelables ne constituent plus un élément complémentaire, mais sont au cœur du système. Il faut donc se demander par quelles mesures le développement des énergies renouvelables doit être complété, et quelles technologies doivent être employées pour cela. En bref, nous avons besoin d'un marché pour les options de flexibilité. Ce nouveau modèle devra être conjugué à la conception de nouveaux secteurs d'activité, initiés aussi bien par les acteurs établis du marché que par les nouveaux arrivants, afin de générer une dynamique propre suffisante. Sur le plan politique également, il faut repenser le système. Fonctionnant souvent en parallèle les unes des autres, les orientations et les lois du système actuel, dit «multiniveaux », doivent se fondre dans une approche intégrée et cohérente, qui aligne et regroupe les intérêts et les possibilités de l'Union européenne, des différents États membres, des régions et des communes. Enfin, ces modifications du système nécessitent un large débat de la société sur les possibilités d'organisation et leurs limites, c'est-à-dire une discussion ouverte et juste sur les pour et les contre des différents scénarios de développement. Seule cette intégration dans le processus d'organisation, alliée à une participation active au changement du système énergétique (par exemple via des centrales électriques citoyennes), permettra à la société d'accepter le processus de transformation. Le tournant énergétique est une mission d'intérêt commun qui exige, pour être remplie avec succès, une «nouvelle» coopération au plan politique, social, scientifique et économique.

POUR CONCLURE, VOICI LES DÉFIS que le tournant énergétique doit relever :

- le défi technologique: intégration progressive des énergies renouvelables dans le système énergétique, développement et utilisation d'options de flexibilité (par exemple systèmes de stockage, systèmes hybrides, maîtrise de la demande en énergie) ;

- le défi de la compatibilité : coopération entre les technologies conventionnelles et nouvelles sur la base d'un modèle adéquat du marché de l'énergie et de l'électricité ;

- le défi de l'investissement : mise à disposition de capitaux suffisants pour transformer le système et garantie de conditions de financement suffisamment attractives ;

- le défi des infrastructures: développement d'infrastructures adaptées pour relier les centres de production aux centres de consommation (par exemple smart grid et super smart grid) ;

- le défi des ressources : lutte contre les effets négatifs sur les ressources (ressources critiques, matières toxiques) ;

- le défi des acteurs : gestion correcte de l'inertie des acteurs établis ; 
- le défi de la société : acceptation sociale de la transformation du système, renforcement de la participation, styles de vie durables, lutte contre les effets de rebond ;

- le défi de la politique : intégration des initiatives politiques régionales, nationales et internationales (approche multiniveaux) dans un cadre politique cohérent ;

- le défi de l'innovation : concentration sur les innovations globales au lieu d'une orientation purement technique (intégration des innovations sociales), tests dans des situations adaptées (laboratoires réels);

- le défi de l'organisation : amélioration des connaissances sur l'organisation des processus de transformation (connaissance des objectifs, des systèmes et des transformations).

Traduction de Marie-Céline GEORG 



\title{
Quelles solutions pour financer la transition énergétique en France ?'
}

\author{
Romain Morel, Hadrien Hainaut, lan COCHRAN
}

Le secteur financier a commencé à s'emparer des questions liées au climat depuis quelques années. C'est une dynamique nouvelle qui répond à une prise de conscience de l'exposition du secteur au changement climatique. Néanmoins, les connaissances sur les enjeux, les impacts et les solutions s'améliorent, mais restent parcellaires. Cet article cherche à dresser un état des lieux des discussions et données disponibles. II illustre également le contexte français, où la question sur le financement de la transition énergétique a connu beaucoup d'avancées ces dernières années.

\section{Les enjeux du financement de la transition énergétique et écologique}

La redirection des investissements nécessaire pour le financement de la transition

Des investissements ambitieux pour atteindre les objectifs climat

En matière de transition énergétique et écologique, les modèles et scénarios prospectifs chargés d'estimer les besoins d'investissement aboutissent à des montants ambitieux. En France, l'ordre de grandeur varie entre 40 et 60 milliards d'euros par $a^{2}$. Au niveau européen, les estimations portent sur des centaines de milliards. Au niveau mondial, sur des milliers de milliards ${ }^{3}$. Ces montants considérables sont à rapporter au volume annuel des investissements dans l'économie, de l'ordre de 400 milliards d'euros en France et de 17000 à 18000 milliards de dollars dans le monde. En proportion, la transition énergétique et écologique mobiliserait donc 5 à $10 \%$ du total des investissements. De plus, ce volume d'investissement est à prévoir quoi qu'il arrive, contrainte climatique ou non. Le « surcoût » d'investissement d'un scénario $2{ }^{\circ} \mathrm{C}$ est un ordre de grandeur audessous : quelques centaines de milliards de dollars par an au niveau mondial.

\section{Le secteur public ne peut pas porter seul tous ces besoins}

Au regard des montants en jeu, l'État ne pourra pas supporter tout seul ces investissements, qui devront également provenir du secteur privé. Ce dernier de-

\footnotetext{
${ }^{1}$ Article rédigé en juin 2015, NdE.

${ }^{2}$ Voir Débat national sur la transition énergétique (DNTE), Rapport du groupe de travail $n^{\circ} 4$ du conseil national. Quels coûts, quels bénéfices et quel financement de la transition énergétique? [en ligne], Paris, 2013. Disponible sur : http://www.developpement-durable.gouv.fr/IMG/pdf/gt4_financement_dnte.pdf [consulté le 10/09/2015].

${ }^{3}$ Voir Agence internationale de l'énergie (AIE), World Energy Investment Outlook - Special Report [en ligne], Paris, 2014. Disponible sur: https://www.iea.org/publications/freepublications/publication/WEIO 2014.pdf [consulté le 10/09/2015].
} 
vra apporter à la fois les capitaux nécessaires, mais aussi sa capacité à mettre en place et mener à bien les projets. Ceci est d'autant plus vrai que la capacité financière du secteur public est aujourd'hui limitée. À ce titre, il doit se concentrer sur ses missions principales et là où il a le plus de leviers : mise en place de signaux clairs aux acteurs de marché, création des cadres d'incitation cohérents, correction des imperfections de marché, atténuation des impacts sociaux de la transition et financement des projets pilotes.

\section{Le secteur privé doit engager un double mouvement}

En complément, le secteur privé peut et doit intervenir partout où les investissements sont importants et rentables. Cette mobilisation implique une augmentation des flux financiers vers les investissements bas carbone et une baisse de ceux destinés aux activités économiques carbonées. Au niveau mondial, les milliers de milliards de dollars à affecter à l'investissement bas carbone ne représentent qu'un faible montant supplémentaire à dégager; ils seront principalement issus de la redirection de plusieurs centaines de milliards, aujourd'hui mobilisés dans des secteurs intensifs en carbone. Ce double mouvement - augmentation et redirection de l'investissement - aura des conséquences profondes sur les ressources publiques et privées.

\section{Les barrières en place à cette redirection}

Aujourd'hui, cette redirection des flux se heurte à des barrières économiques. Le cadre fiscal de notre économie continue d'encourager les investissements carbonés. Le coût des émissions de $\mathrm{CO}_{2}$ n'est pas représenté dans le prix payé par les consommateurs qui achètent ces énergies ou décident d'investir dans des biens ou équipements qui les consomment. Plusieurs études récentes ont cherché à démontrer l'ampleur des "subventions aux énergies fossiles », soit versées directement, soit indirectement par la différence entre les coûts sociaux et les coûts réels de ces énergies. Récemment, le Fonds monétaire international (FMI) a estimé leur coût budgétaire direct à 500 milliards de dollars par an. Au niveau mondial, les subventions considérées sont le plus souvent des subventions à la consommation d'énergie, comme les tarifs sociaux. En intégrant le coût des externalités, le coût global de ces subventions est estimé à 5000 milliards de dollars par an.

Les barrières à l'investissement bas carbone sont également politiques. Malgré la volonté des États d'agir sur le changement climatique et de soutenir l'investissement, des incohérences de fond subsistent. À côté des politiques climatiques, les États continuent de financer ou soutenir des secteurs carbonés. Il en résulte un manque de cohérence qui brouille le signal de long terme que les pouvoirs publics souhaitent donner. Pour remédier à cette situation, il est indispensable de continuer à distiller les enjeux et les politiques du climat dans tous les domaines de l'intervention publique. Cet alignement des initiatives pu- 
bliques doit également servir à clarifier, vis-à-vis des acteurs privés, l'ampleur et les rythmes des changements en cours ${ }^{4}$. En effet, la variabilité et la mauvaise lisibilité de la politique climatique sont des obstacles majeurs au développement des investissements. Aujourd'hui, le «fossé » d'investissement privé entre les besoins d'investissements «climato-compatibles » et les investissements existants est l'illustration de la faible qualité du signal donné par les pouvoirs publics : les acteurs perçoivent ce manque de cohérence et donnent ainsi peu de crédit aux objectifs de long terme.

\section{Le besoin d'une approche systémique}

Le besoin de rendre le climat mainstream est particulièrement criant au sein du système financier ${ }^{5}$. Si l'intégration des enjeux climatiques dans le secteur financier a progressé récemment, cette dynamique reste marginale. Les règles prudentielles, la manière de prendre en compte les risques liés aux projets financés et les durées considérées pour la rentabilité des investissements sont, par exemple, globalement défavorables aux trajectoires à faible intensité de carbone. Or la transition énergétique ne peut se faire sans inclure la manière dont sont prises les décisions d'investissement.

Le changement climatique fait naître une nouvelle gamme de risques pour les entreprises et les institutions financières. En plus des risques directement liés à l'exposition de l'activité aux effets du climat (évènements extrêmes, conditions de température, risque côtier, etc.), le risque régulatoire est passé au premier plan des préoccupations. C'est le principe des «stranded assets » ou actifs échoués. Appliqué au secteur de l'extraction des énergies fossiles, une majorité des réserves connues d'énergies fossiles ne vont pas pouvoir être exploitées car elles mettraient en péril le budget carbone compatible avec les objectifs à long terme. Au-delà de la contrainte sur l'utilisation des réserves, la logique des stranded assets a des implications pour le système financier, notamment dans la manière dont sont évalués et valorisés les actifs des sociétés et des États. Ces risques pourraient ainsi s'avérer systémiques et mettre en péril la stabilité financière. À ce titre, les institutions chargées de cette stabilité devraient commencer à intégrer la problématique climat dans le cadre de leur mandat historique (Morel et al., 2015).

La complexité des circuits financiers et le caractère transversal de la transition énergétique et écologique appellent une approche systémique, capable de

\footnotetext{
${ }^{4}$ Organisation de coopération et de développement économiques (OCDE), Aligning Policies for the Transition to a Low-Carbon Economy [en ligne], Paris, 2015 Disponible sur: http://www.oecd.org/ $\mathrm{mcm}$ /documents/Aligning-policies-for-the-transition-to-a-low-carbon-economy-CMIN2015-11.pdf [consulté le $10 / 09 / 2015]$

${ }^{5}$ Morel et al., Mainstreaming Climate Change in the Financial Sector and its Governance [en ligne], CDC Climat/Institut du développement durable et des relations internationales (Iddri), Paris, 2015. Disponible sur : http://www.i4ce.org/download/lintegration-du-changement-climatique-dans-le-secteur-financier-et-sa-gouver nance/ [consulté le 07/10/2015].
} 
maintenir une cohérence globale dans l'intervention publique. D'un secteur à l'autre, les besoins, volumes et mécanismes d'intervention des acteurs publics et privés diffèrent fortement. Il faut donc élaborer un mix de politiques qui puisse s'appliquer de manière efficace pour chacun des acteurs. Dans cette perspective, il n'est pas raisonnable d'attendre un outil omnipotent puisque chaque acteur a ses problématiques qui appellent un traitement adéquat.

\section{Les initiatives françaises sur le financement de la transition}

En France, les besoins d'investissement au titre de la transition énergétique sont évalués entre 40 et 60 milliards d'euros par an, jusqu'en 2050. Là encore, ces chiffres considérables doivent être renvoyés aux ordres de grandeur des investissements globaux dans l'économie, et comparés aux investissements qu'il est de toute façon nécessaire de réaliser en raison du renouvellement de nos équipements de production et de consommation d'énergie. Plusieurs initiatives récentes se sont consacrées à l'identification des moyens de financer ces investissements.

\section{Le livre blanc sur le financement de la transition écologique : poser des bases}

En 2013, le livre blanc sur le financement de la transition écologique marque l'importance prise par le sujet du financement dans la foulée du Débat national sur la transition énergétique (DNTE). Ce document, réalisé par Dominique Dron et Thierry Francq sur commande de la direction générale du Trésor et du Commissariat général au développement durable (CGDD), fait l'état des lieux de la problématique du financement et propose quatre principes fondamentaux pour mobiliser efficacement les financements :

- l'amélioration de la prévisibilité des signaux fournis, dans le contexte de clarification et de cohérence de la politique publique évoqué plus haut ;

- la création d'outils ciblés, en complément des instruments existants, pour mobiliser les financements publics et privés. Ces instruments doivent notamment permettre un partage du risque entre public et privé ;

- la prise en compte des enjeux extra-financiers, appelés aussi critères $\mathrm{ESG}^{6}$, pour garantir que toutes les composantes de la transition sont prises en compte. Les instruments comme la conditionnalité ou la traçabilité des flux financiers sont fondamentaux à cette fin ;

- une évolution du cadre intellectuel des pratiques des acteurs du financement, au moyen d'outils de formation et de sensibilisation plus répandus.

\footnotetext{
${ }^{6}$ Environnement, social et gouvernance.
} 
Le livre blanc pose les bases pour les réflexions ultérieures, notamment en matière de financement de la transition énergétique.

La conférence bancaire et financière : un premier rassemblement d'acteurs

À l'été 2014 s'est tenue une conférence bancaire et financière de la transition énergétique, dans le prolongement du débat national et au moment de l'élaboration du projet de loi actuellement en débat. Cette conférence s'est penchée sur trois points principaux :

- les travaux de rénovation des logements particuliers. Il s'agissait notamment de discuter des mesures de simplification des instruments existants comme l'éco-PTZ ou le tiers-financement, et la création d'un fonds de garantie soutenant des taux d'emprunt avantageux pour les ménages qui réalisent des travaux ;

- le financement des entreprises, en particulier au travers de la création d'obligations vertes, financements de long terme pour des entreprises qui les consacrent à des investissements favorables à la transition énergétique. Les investisseurs institutionnels seraient encouragés à détenir une part plus importante de ces obligations ;

- les aides aux collectivités, avec notamment un rôle de prêteur renforcé pour la Caisse des Dépôts.

Si la conférence bancaire et financière s'est tenue de manière relativement discrète et essentiellement sur l'optimisation de dispositifs existants, elle n'en a pas moins constitué une première étape du dialogue entre les parties prenantes du financement sur le thème de la transition énergétique.

\section{Le PLTECV : des avancées sur le reporting}

Le projet de loi pour la transition énergétique et la croissance verte (PLTECV) constitue une initiative majeure en France en matière de transition énergétique. Le projet de loi est élaboré à partir du débat national qui avait été engagé dès 2012 sur ce sujet. À l'heure actuelle, le projet de loi est toujours en cours d'évolution au fil du processus législatif établi entre les deux chambres du Parlement.

En termes de financement, le projet de loi concrétise plusieurs mesures comme la simplification des dispositifs de prêt et le renforcement du crédit d'impôt pour les travaux des particuliers dans la rénovation des logements. Il comprend également un volet destiné au compte rendu des flux financiers dans les sociétés privées (financières et non financières). La notion d'empreinte carbone des actifs financiers ferait donc son entrée dans la loi, obligeant les sociétés à monter en compétence sur la mesure et les implications de ce type d'indicateur. La part verte, c'est-à-dire la proportion des actifs détenus contribuant au processus de transition, devient également un indicateur clé, sur lequel des ob- 
jectifs chiffrés ( $5 \%$ en 2016, $10 \%$ en 2020) sont proposés pour les institutions financières.

Si les mesures annoncées par le projet de loi contribuent à renforcer la gamme des instruments disponibles, elles ne résolvent pas l'enjeu de la cohérence globale de la politique publique. Il manque un cadre réglementaire cohérent pour mobiliser efficacement les flux privés. D'une manière générale, une partie des questions liées au financement devraient être abordées dans les projets de loi de finances.

\section{La stratégie nationale bas carbone}

Élaborée en parallèle du projet de loi, la stratégie nationale bas carbone (SNBC) est un document de référence destiné à mettre en cohérence l'action publique en matière de transition énergétique. La stratégie détaille les trajectoires de chaque secteur et identifie le rôle et l'impact des principaux instruments de la politique publique. Des «budgets carbone», par secteur et période, devraient permettre d'anticiper les efforts de réduction et de proposer les outils adéquats pour les mettre en œuvre. Il est prévu d'approfondir ces trajectoires par des évaluations économiques des volumes d'investissements à mettre en œuvre.

L'élaboration d'une stratégie nationale bas carbone est un élément indispensable pour renforcer la lisibilité et la stabilité de la politique publique en matière de transition énergétique et écologique. En revanche, elle ne couvre pas spécifiquement les outils financiers associés à la transition.

\section{L'analyse des flux financiers pour le climat en France}

Pour répondre au besoin d'une approche systémique de la finance climat, CDC Climat Recherche travaille depuis début 2014 sur un Panorama des financements climatiques. Cette étude est la déclinaison française d'une initiative portée par l'organisation non gouvernementale (ONG) Climate Policy Initiative (CPI). Des travaux similaires ont été conduits en Allemagne et au niveau mondial.

Une méthode mondialement reconnue

L'objectif de l'étude est de recenser les financements pour le climat, c'est-à-dire les financements des équipements tangibles qui réduisent les émissions de gaz à effet de serre. L'étude couvre aussi les financements pour l'adaptation, bien que la définition de ce périmètre soit plus ouverte et que l'information au sujet des flux financiers soit parfois manquante.

\section{Le périmètre de l'étude}

La méthode déployée se base sur les directives établies par la CPI et les applique au contexte français. Concrètement, l'étude ne considère que les investis- 
sements dans les infrastructures et équipements tangibles, pour lesquels le lien entre l'investissement et la baisse des émissions est clairement établi. Les dépenses de fonctionnement, de recherche et développement, de production de matériaux, de formation et d'information ne sont donc pas considérées, malgré leur importance pour la transition.

L'étude considère trois grandes catégories d'actions : l'efficacité énergétique, les capacités de production ou de consommation d'énergies renouvelables, et les équipements de transport «durables », notamment les infrastructures. Ces actions se déclinent dans différents secteurs : bâtiment, transport, industrie, agriculture, énergie. Certaines catégories «à part », comme le nucléaire ou les flux internationaux, sont également considérées, ce qui permet de les inclure ou non dans le résultat final selon les besoins.

L'étude caractérise les investissements, c'est-à-dire les volumes engagés dans les équipements, et documente la nature des flux financiers mobilisés pour réaliser ces investissements. Différentes méthodes sont juxtaposées. Pour certains secteurs, l'étude considère la totalité de l'investissement, pour d'autres, seulement le coût marginal de l'efficacité énergétique (par exemple, dans le bâtiment neuf).

Il existe plusieurs manières de définir les investissements «climat». La Convention-cadre des Nations unies sur les changements climatiques (CCNUCC), au travers de son comité permanent sur le financement (Standing Committee on Finance, SCF) retient que le financement dans la lutte contre les changements climatiques «vise à réduire les émissions, à renforcer les puits de gaz à effet de serre, à réduire la vulnérabilité et à maintenir et accroître la résilience des systèmes humains et écologiques face aux impacts négatifs du changement climatique ». D'autres approches sont possibles, mais dans les faits, c'est au niveau de chaque secteur, voire du contexte national, que se fait la sélection des types de projets retenus au titre du climat.

\section{Vue d'ensemble des financements en France}

Selon l'étude Panorama des financements climatiques en France en 2011', entre 20 et 25 milliards d'euros ont été consacrés aux investissements climat en France. L'écart entre ces deux chiffres provient essentiellement de l'inclusion ou non des secteurs annexes, comme le nucléaire ou certaines infrastructures de transport. De cet ensemble, au moins 5 milliards sont directement mobilisés par le secteur public, dont la moitié dans le domaine des transports durables. La finance privée, elle, se concentre dans les secteurs de l'industrie et de l'énergie. Le secteur du bâtiment représente 7 milliards d'euros d'investissements dans l'efficacité énergétique et 3 milliards d'euros dans les énergies renouvelables.

\footnotetext{
${ }^{7}$ Romain Morel, Ian Cochran, Romain Hubert, Jeanne Dequesne (CDC Climat Recherche) et Morgan HervéMignucci (Climate Policy Initiative), Panorama des financements climatiques en France en 2011, CDC Climat Recherche, Paris, octobre 2014.
} 
Tableau 1 : Récapitulatif des financements dédiés au climat en France en 2011

\begin{tabular}{|c|c|c|c|}
\hline \multirow[b]{2}{*}{ France, 2011} & \multirow{2}{*}{$\begin{array}{c}\text { Total des } \\
\text { financements climat } \\
\text { (en milliards d'euros) }\end{array}$} & \multicolumn{2}{|c|}{ Sources de financement } \\
\hline & & $\begin{array}{l}\text { Part du secteur } \\
\text { public (\%) }\end{array}$ & $\begin{array}{l}\text { Part du secteur } \\
\text { privé (\%) }\end{array}$ \\
\hline Bâtiment & 10,1 & $36 \%$ & $64 \%$ \\
\hline Transports*) & 2,6 & $100 \%$ & $0 \%$ \\
\hline Industrie ${ }^{\star \star}$ ) & 2,1 & $0 \%$ & $100 \%$ \\
\hline Agriculture & 0,8 & $16 \%$ & $84 \%$ \\
\hline Énergie & 4,4 & $9 \%$ & $91 \%$ \\
\hline Total & 20,0 & $34 \%$ & $66 \%$ \\
\hline Nucléaire & 0,9 & $0 \%$ & $100 \%$ \\
\hline International $\left.\right|^{* * \star}$ ) & 7,2 & $76 \%$ & $24 \%$ \\
\hline Ensemble & 28,1 & $44 \%$ & $56 \%$ \\
\hline
\end{tabular}

Source : Romain Morel, Ian Cochran, Romain Hubert, Jeanne Dequesne (CDC Climat Recherche) et Morgan Hervé-Mignucci (Climate Policy Initiative), Panorama des financements climatiques en France en 2011, CDC Climat Recherche, Paris, octobre 2014. *) Dans le secteur des transports, ne sont pas comptabilisées les participations des entreprises dans les partenariats public-privé (PPP) ; un périmètre plus large incluant des infrastructures de transport dont l'impact climatique est plus indirect représente un peu moins de 7 milliards d'euros; **) dans le secteur de l'industrie, la part des financements publics n'a pas pu être mise en évidence ; ***) les montants privés présentés sont issus du fDi Report, dont le périmètre ne correspond pas totalement à celui du reste de l'étude.

Au-delà de la vision d'ensemble, ce type d'étude doit aussi permettre de faire la typologie des acteurs qui réalisent les investissements. En effet, la nature des instruments efficaces dépend beaucoup du mode d'organisation des acteurs réalisant les dépenses. Par exemple, la création d'un fonds pour soutenir l'investissement ne se justifie que si les dépenses sont relativement concentrées sur un faible nombre d'acteurs. Dans un secteur diffus, comme le logement où interviennent plusieurs centaines de milliers de propriétaires réalisant des travaux, ce type d'instrument est peu adapté. L'étude en 2011 montre qu'il y a une parité entre le financement de gros et le financement de détail, tous secteurs confondus. Il existe cependant des disparités : le financement des énergies renouvelables est plus concentré sur l'année 2011, celui de l'efficacité énergétique plus diffus.

L'année 2011, pour laquelle a été réalisée cette première édition du Panorama, est une année «haute» en ce qui concerne le financement des énergies renouvelables. Par la suite, les prix ont baissé, notamment sur l'énergie solaire, et on constate un ralentissement des volumes de puissance installée. Pour mieux comprendre ces évolutions, CDC Climat Recherche travaille en ce moment sur une extension du Panorama pour les années 2012 à 2014.

\section{Comparaison France-Allemagne}

Dans le cadre de la comparaison des transitions énergétiques française et allemande, il est intéressant de mettre en perspective le Panorama réalisé en France 
avec celui produit par la CPI pour l'Allemagne en 2012 sur l'année 2010. Au niveau des volumes d'ensemble, la France consacre une plus faible part de son produit intérieur brut (PIB) aux investissements dédiés au climat, de l'ordre de $1 \%$, contre $1,5 \%$ en Allemagne. La différence est uniquement due aux énergies renouvelables ainsi qu'à leur acheminement.

C'est en matière d'instruments que les deux pays divergent le plus. La France affiche un montant élevé de subventions directes (de l'ordre de 3 milliards d'euros), tandis qu'en Allemagne l'intervention publique repose principalement sur la distribution de prêts concessionnels ${ }^{8}$. Cette seconde approche présente l'avantage d'afficher un effet de levier beaucoup plus important. Cette différence de philosophie et de mise en œuvre des moyens de soutien à la transition énergétique est source d'un important débat en France sur la manière dont le modèle allemand peut inspirer des évolutions dans les dispositifs publics. Cette question porte à la fois sur l'effet de levier, mais aussi sur la dissémination de l'expertise sur les financements climat. En effet, en Allemagne, la banque publique Kreditanstalt für Wiederaufbau (KfW) s'appuie sur le réseau bancaire existant tout en lui apportant une expertise technique fine sur les projets concernés.

\section{Conclusion : des ordres de grandeur gérables qui appellent à la confiance et à l'action}

Il faut rapprocher les volumes d'investissement recensés dans le cadre d'exercices comme le Panorama, de l'ordre de 20 à 25 milliards d'euros par an, avec les ordres de grandeur des besoins nécessaires à la transition, de 40 à 60 milliards d'euros par an pour la France. Ce rapport de 1 à 2 est assez représentatif de la situation actuelle en matière de financement des investissements ${ }^{9}$. Le double mouvement à engager du côté de la finance privée, et notamment la redirection des flux qui vont aujourd'hui aux investissements carbonés, constitue la clé pour combler l'écart qui nous sépare de l'atteinte des objectifs de la transition énergétique et écologique.

Cela montre que les objectifs d'investissement sont ambitieux, mais gérables. Cela appelle donc à retoquer toute forme de défaitisme vis-à-vis des enjeux, mais également à engager rapidement les actions nécessaires pour combler le fossé actuel entre besoins et financements existants. À ce titre, même si le rôle du secteur privé est central, les pouvoirs publics ont un rôle clé à jouer, notamment dans la fourniture d'un signal fort et cohérent.

\footnotetext{
${ }^{8}$ L'étude allemande se concentre sur le niveau fédéral. Il est possible qu'en intégrant les politiques régionales, le rôle des subventions soit plus important.

${ }^{9}$ Ce point est renforcé par le fait que le périmètre des besoins estimés est plus large que le périmètre de l'étude sur les financements climatiques.
} 



\section{Comment financer la transition énergétique en Allemagne ? ${ }^{1}$}

\section{Ulrich BENTERBUSCH}

En guise d'introduction, j'aimerais rapidement présenter l'Agence allemande de l'énergie (Deutsche Energie-Agentur, dena). Elle occupe une place particulière dans le paysage des institutions et autorités allemandes. La dena a été créée il y a 15 ans à Berlin. Cette société de projet s'est depuis inspirée du concept de partenariat public-privé : elle est présente sur le marché en tant que société privée, et propose des prestations de services à des partenaires politiques et économiques pour l'organisation et la mise en œuvre des objectifs du tournant énergétique (Energiewende). À peu près la moitié de son budget annuel (d'environ 20 millions d'euros) provient de partenaires économiques, tandis que l'autre moitié est constituée de fonds publics, en particulier octroyés par les ministères fédéraux. La dena emploie 185 personnes. Par rapport à l'Agence de l'environnement et de la maîtrise de l'énergie (ADEME), c'est relativement peu. Cependant, il faut tenir compte du fait qu'en République fédérale d'Allemagne, il existe également des agences de l'énergie au niveau des Länder, de sorte que, l'un dans l'autre, ces structures sont équivalentes.

La mission de la dena consiste à soutenir le gouvernement dans l'organisation et la mise en œuvre du tournant énergétique. En matière d'efficacité énergétique, par exemple, elle est toujours intervenue pour développer les marchés. II ne s'agit pas tant d'injecter de l'argent public dans des projets que de créer les conditions nécessaires au fonctionnement du marché privé. La dena est compétente et active dans tous les domaines importants de la transition énergétique. Cela inclut aussi bien le système électrique dans son ensemble et la question de l'intégration des énergies renouvelables dans ce système que l'efficacité énergétique, en particulier dans le secteur du bâtiment, dans l'électricité et les transports.

Pour commencer, je vais brièvement revenir sur le contexte de la transition énergétique. J'aborderai ensuite la question du financement dans deux domaines: celui de l'efficacité énergétique, et celui des énergies renouvelables et des systèmes énergétiques.

\section{Le contexte de la transition énergétique}

Lorsqu' on parle du financement de la transition énergétique, il faut d'abord se faire une idée des objectifs et des champs d'action de la politique énergétique et climatique en Allemagne. Dans quel contexte politique se présente donc le financement de la transition énergétique en Allemagne?

Réduction des émissions de $\mathrm{CO}_{2}$ :

l'efficacité énergétique et les énergies renouvelables sont les principaux facteurs

L'objectif principal de la politique mondiale en matière de climat est de limiter à $2{ }^{\circ} \mathrm{C}$ le réchauffement climatique. Pour cela, il faut nettement réduire les émis-

\footnotetext{
${ }^{1}$ Texte issu d'une communication donnée dans le cadre de la journée d'étude Les politiques énergétiques française et allemande des années 1970 à la transition énergétique, coorganisée par le CIRAC le 13 avril 2015, NdE.
} 
sions de $\mathrm{CO}_{2}$. L'Agence internationale de l'énergie (AIE) a déterminé par quelles mesures nous pourrions suffisamment réduire les émissions de $\mathrm{CO}_{2}$ d'ici 2035 pour que le réchauffement climatique soit limité à $2{ }^{\circ} \mathrm{C}$. Le principal levier est l'efficacité énergétique : celle-ci contribue pour $44 \%$ à la réalisation de cet objectif. Si l'on inclut les moteurs alternatifs, on arrive même à $47 \%$. Ces chiffres coïncident avec les analyses à long terme concernant l'Allemagne, selon lesquelles l'efficacité énergétique pourrait contribuer pour plus de $50 \%$ à la réduction des émissions de $\mathrm{CO}_{2}$ dans le pays d'ici 2050, en particulier dans le secteur de la chaleur - c'est-à-dire l'eau chaude pour les ménages, le chauffage, mais aussi le chauffage industriel. Avec un taux de $23 \%$, le deuxième levier d'action déterminé par l'AIE réside dans le développement des énergies renouvelables; en incluant les biocarburants, il représente même $27 \%$. Viennent ensuite le captage et le stockage de dioxyde de carbone (en anglais carbon capture and storage, CCS) contribuant pour $17 \%$ à la réalisation de l'objectif, et l'énergie nucléaire, pour $8 \%$. Ces chiffres montrent bien que si nous voulons obtenir des résultats, nous devons nous préoccuper d'abord de l'efficacité énergétique. En effet, moins nous consommons de kilowattheures, moins nous devons produire ou exploiter de précieuses sources d'énergie comme l'électricité ou le gaz. Pourtant, l'importance de l'efficacité énergétique est souvent sous-estimée.

Les objectifs du gouvernement fédéral allemand en matière de politique énergétique

Dans son projet énergétique (Energiekonzept), le gouvernement fédéral formule des lignes directrices pour une stratégie globale s'étendant jusqu'en 2050, avec les objectifs essentiels suivants :

- réduire les émissions de gaz à effet de serre de $40 \%$ d'ici 2020 et de $80 \%$ d'ici 2050 (par rapport à 1990) ;

- réduire la consommation d'énergie primaire de $20 \%$ d'ici 2020 et de $50 \%$ d'ici 2050 (par rapport à 2008);

- augmenter la part des énergies renouvelables dans la consommation brute d'énergie finale pour atteindre $18 \%$ d'ici 2020 et $60 \%$ d'ici 2050 ;

- augmenter la part de l'électricité produite à partir d'énergies renouvelables dans la consommation brute d'électricité jusqu'à 40-45\% d'ici 2025, 55-60\% d'ici 2035 et $80 \%$ d'ici 2050.

L'Union européenne (UE), pour sa part, se concentre sur trois grands indicateurs d'ici 2030 :

- réduire les gaz à effet de serre de 40 \% par rapport à 1990 ;

- développer les énergies renouvelables pour atteindre au moins $27 \%$ de la consommation d'énergie ;

- réduire la demande d'au moins $27 \%$ (objectif indicatif).

Aucun État membre de l'UE n'est en voie d'atteindre ces trois objectifs, le moins avancé étant celui de l'efficacité énergétique. 


\section{Statu quo dans les différents domaines d'action}

Le rapport d'avancement sur la transition énergétique présenté par le gouvernement fédéral pour la première fois en décembre 2014 montre que l'objectif de développement des énergies renouvelables est le seul à avoir été atteint et même dépassé dans ce cadre, mais à un coût très élevé. Malgré la réforme de la loi sur les énergies renouvelables (Erneuerbare-Energien-Gesetz, EEG) adoptée en 2014, l'Allemagne n'a pas encore réussi, par exemple, à mettre l'énergie éolienne sur la voie d'un développement modéré.

Dans tous les domaines (demande en électricité, demande en chaleur, énergies renouvelables, centrales électriques, réseau de transport, réseau de distribution, réseau offshore, stockage et conception du marché), la dena pense qu'il est urgent d'agir. La mise en œuvre de la transition énergétique est complexe et difficile. Il faut poser dès maintenant les jalons d'une transition énergétique réussie. Le principal aspect consiste à coordonner le développement des énergies renouvelables avec la progression réalisée dans les autres domaines.

Parmi les principales tâches, on peut citer :

- la coordination du développement des réseaux avec le développement des énergies renouvelables ;

- la diminution de la demande en énergie (c'est-à-dire l'efficacité énergétique). En matière d'efficacité énergétique dans le domaine de la chaleur et dans celui des transports - en particulier des véhicules utilitaires -, les résultats à long terme sont loin d'être garantis, même si l'Allemagne s'est fixé des objectifs très ambitieux pour la réduction des gaz à effet de serre. L'avis des experts qui ont commenté le rapport d'avancement sur demande du gouvernement fédéral n'est pas favorable sur ce point ;

- la création d'une nouvelle conception du marché de l'électricité.

Nous devons garder cette situation de départ à l'esprit lorsque nous nous intéressons à la question du financement. En effet, chaque champ d'action pose à sa manière la question de savoir comment financer les mesures correspondantes.

\section{Le financement dans le domaine de l'efficacité énergétique}

Comme on l'a dit plus haut, l'efficacité énergétique est de loin le plus important levier pour atteindre les objectifs climatiques. En même temps, c'est le champ d'action dans lequel il est le plus difficile d'atteindre les objectifs fixés, en Allemagne comme dans bien d'autres pays d'Europe, notamment parce que les mesures sont par nature beaucoup plus difficiles à mettre en œuvre dans ce domaine, à cause de la multiplicité des acteurs et des différents sous-domaines concernés.

C'est pourtant précisément dans ce domaine que les mesures sont généralement les plus attractives économiquement : les investissements sont remboursés par les économies d'énergie et les rendements sont souvent plus élevés que 
sur le marché des capitaux. De plus, les réussites dans l'amélioration de l'efficacité énergétique ont des répercussions sur la coûteuse production d'énergie : chaque kilowattheure non consommé n'a pas besoin d'être produit !

De ce point de vue, désigner l'efficacité énergétique comme «le deuxième pilier du tournant énergétique » (après les énergies renouvelables) relève de l'euphémisme. C'est néanmoins sous ce slogan que le gouvernement fédéral a donné fin 2014 le signal de départ pour progresser plus vite dans ce domaine.

\section{NAPE : un plan d'action national pour l'efficacité énergétique}

Au cœur de la politique visant à augmenter l'efficacité énergétique se trouve le plan d'action national pour l'efficacité énergétique (Nationaler Aktionsplan Energieeffizienz, NAPE). Le gouvernement fédéral l'a présenté en décembre 2014, en même temps que son programme d'action 2020 pour la protection du climat (Aktionsprogramm Klimaschutz 2020).

Il s'agit d'une stratégie visant à augmenter l'efficacité énergétique d'ici 2020, en incluant la mise en œuvre de la directive européenne sur l'efficacité énergétique, sous le triple slogan « Informer - Promouvoir - Exiger ». Elle prévoit d'agir dans quatre directions :

- l'efficacité énergétique dans les bâtiments ;

- les économies d'énergie, un modèle de performance économique et d'entreprise ;

- la responsabilité individuelle en matière d'efficacité énergétique ;

- la poursuite des mesures relatives aux transports.

Pour ces quatre champs d'action, le gouvernement fédéral a défini 14 mesures immédiates ainsi que 22 processus d'approfondissement. Il a notamment créé une plate-forme pour l'efficacité énergétique, afin de développer les perspectives de travail à long terme dans ce domaine pour les différents acteurs du monde économique, de la société civile, de la sphère scientifique, ainsi que des ministères et des Länder concernés.

Le NAPE s'appuie souvent sur des instruments de financement existants, en les perfectionnant et en les développant. Cependant, avant de «promouvoir », il faut «informer». On ne devrait pas avoir à subventionner quelque chose qui rapporte déjà en soi. L'objectif principal doit être de donner une impulsion aux marchés pour l'efficacité énergétique. Les subventions ne sont donc pas toujours le levier décisif, mais constituent une motivation efficace.

\section{Promotion de l'efficacité énergétique dans les bâtiments}

Le NAPE a pour but de perfectionner et de renforcer les instruments incitatifs dans le secteur du bâtiment.

Les subventions d'État pour les conseils sur site sont par exemple passées de $50 \%$ à $60 \%$. Pour une maison individuelle ou jumelée, l'aide peut désor- 
mais aller jusqu'à 800 euros. En 2014, l'État fédéral a ainsi financé des conseils sur site à hauteur de 3 millions d'euros au total.

L'enveloppe du programme d'amélioration de la performance énergétique des bâtiments mis en place par la banque publique Kreditanstalt für Wiederaufbau (KfW), littéralement « établissement de crédit pour la reconstruction », qui encourage l'efficacité énergétique dans l'immobilier neuf ou la rénovation par des subventions ou des prêts à taux bonifié, est passée de 1,8 à 2 milliards d'euros par an. Cependant, ce n'est pas seulement le volume des moyens mis à disposition qui importe ; c'est aussi le volume effectivement dépensé. À ce niveau, la situation est un peu moins réjouissante. L'effet incitatif des réductions de taux par la KfW n'est visiblement pas très élevé. C'est pourquoi, dans les semaines et les mois qui viennent, nous devons nous demander comment rendre ce programme plus attractif.

Le programme incitant le marché à recourir aux énergies renouvelables, qui doit être perfectionné dans le cadre de la stratégie d'efficacité énergétique du bâtiment, a également vu ses moyens augmenter et s'élève désormais à 300 millions d'euros par an au total. Il consiste en des subventions pour les ménages et les entreprises qui veulent utiliser des énergies renouvelables pour se chauffer. Si on veut réduire les émissions de carbone liées à la production de chaleur, il est très important de se demander comment on peut intégrer les énergies renouvelables dans le marché de la chaleur.

C'est peut-être - y compris dans une perspective d'avenir - la question la plus passionnante, car la transition énergétique dans le secteur de l'électricité va entraîner, dans les prochaines décennies, la présence de plus en plus fréquente d' «excédents de courant», c'est-à-dire d'une quantité superflue d'électricité dans le réseau, produite lorsque le vent souffle fort et que le soleil brille franchement. Nous devons donc trouver des solutions afin que le marché de la chaleur, dans sa structure future, soit compatible avec ce système de production d'électricité à partir d'énergies renouvelables. Un exemple, qui constitue dès aujourd'hui une solution possible, est la conversion d'électricité en chaleur, le Power-to-Heat. De nombreuses régies municipales (Stadtwerke) construisent de grands réservoirs d'eau, afin de mieux s'adapter à la transition énergétique au moyen de la cogénération. Ce sont des approches qui pourront certainement nous aider à l'avenir.

Un outil qui n'a, à ce jour, pas encore été mis en œuvre au plan politique est l'incitation fiscale à investir dans des mesures de rénovation énergétique pour les propriétaires de maisons individuelles ou jumelées. C'est fort regrettable, car les propriétaires de ces maisons sont une cible que les programmes de la KfW n'ont pas encore réussi à toucher efficacement, du moins pas en masse. De plus, c'est un groupe qui réagit normalement très fortement aux incitations fiscales. Dans le système fédéral allemand, les ministres des Finances des Länder prennent part au processus de décision au sein du Bundesrat. Malheureusement, jusqu'à présent, les nombreuses tentatives pour faire passer des mesures d'inci- 
tation fiscale se sont toujours heurtées au veto du Bundesrat. Même la dernière, après l'adoption du NAPE, a échoué.

Le gouvernement fédéral va désormais s'assurer que le milliard d'euros de subventions planifié sur cinq ans sera dirigé vers d'autres programmes afin de générer d'autres effets incitatifs. À la dena, nous avons milité pour l'incitation fiscale, car c'est un instrument très efficace. Selon nos calculs - dont les résultats sont largement partagés par le milieu scientifique -, un euro d'impôt non perçu génère huit euros d'investissements. Avec ces huit euros, l'incitation fiscale est plus que remboursée à l'État, en particulier par la TVA.

\section{Les économies d'énergie, un modèle de performance économique et d'entreprise}

La dena pense depuis longtemps que l'efficacité énergétique doit aussi se comprendre comme un modèle de performance économique et d'entreprise. Le NAPE renforce désormais ce type de modèles d'entreprise au-delà du secteur du bâtiment.

Une nouvelle approche, prometteuse, est celle des appels d'offres publics pour des mesures d'efficacité dans le domaine de l'électricité. Tous les acteurs possibles peuvent y participer : prestataires de services énergétiques, régies municipales, coopératives énergétiques, etc. Plus les économies d’énergie par « euro d'incitation » sont importantes, plus les subventions sont élevées. Le montant des fonds s'élève à 300 millions d'euros d'ici 2018.

Le modèle de «contracting » doit lui aussi être renforcé et rendu plus attractif, en particulier pour les petites et moyennes entreprises (PME). Il concerne la fourniture d'énergie ou les économies d'énergie. Des prestataires privés proposent à une entreprise un contrat aux termes duquel ils s'engagent à assurer l'approvisionnement et l'exploitation énergétiques d'un bâtiment ou d'une entreprise, en tirant leurs bénéfices des économies réalisées. Le client y trouve aussi son avantage, sans avoir à se préoccuper de la mise en œuvre. Le contracting est également un instrument très important pour aider les villes et les communes à économiser l'énergie. Nos modélisations ont montré qu'il serait possible d'économiser $30 \%$ des coûts énergétiques sans gros problèmes par le biais de ces contrats.

Ainsi, l'important n'est pas toujours de savoir combien d'argent l'État peut mettre à disposition. Si le cadre des investissements pour l'efficacité énergétique est correctement posé, on peut aussi exploiter les forces du marché pour que l'attrait des économies entraîne la réalisation de nombreux investissements. Le contracting est un modèle que nous n'avons pas encore suffisamment développé, mais je suis sûr que dans les années qui viennent, nous allons accomplir de nets progrès. Par exemple, nous avons besoin de plus de garanties de bonne fin. Jusqu'à présent, nous avons eu beaucoup de problèmes avec ces garanties dans les contrats d'économies d'énergie, mais c'est une question que nous allons régler. En outre, il subsiste quelques autres problèmes juridiques. Une fois que nous les aurons résolus, nous pourrons libérer les forces du marché. 
Le NAPE prévoit également de mettre en place des crédits KfW pour l'efficacité énergétique dans les installations et processus de production, dont la hauteur dépendra de l'ampleur des économies d'énergie réalisées. Il existe déjà toute une série d'incitations à l'efficacité énergétique dans les entreprises, notamment pour :

- les prestations de conseil en énergie (jusqu'à $80 \%$ des coûts pris en charge pour la consultation initiale);

- la certification des systèmes de gestion de l'énergie (jusqu'à $80 \%$ ) ;

- la certification d'un système de contrôle énergétique (jusqu'à 80 \%);

- les investissements dans des technologies transversales d'efficacité énergétique (par exemple air comprimé ou pompes, jusqu'à $30 \%$ ) ;

- les mesures d'optimisation énergétique des systèmes (jusqu'à $30 \%$ ).

S'y ajoute le système de remboursement partiel des taxes sur l'énergie et l'électricité pour les plus gros consommateurs dans l'industrie manufacturière (Spitzenausgleich) $)^{2}$. L'objectif de ces mesures est d'abolir, à moyen terme, les obstacles au contracting et aux investissements dans l'efficacité énergétique.

Les potentiels d'économies d'énergie

\section{Graphique 1 : Consommation d'énergie finale en 2008 (en pétajoules) et potentiels d'économies d'énergie d'ici 2020 (en \%)}

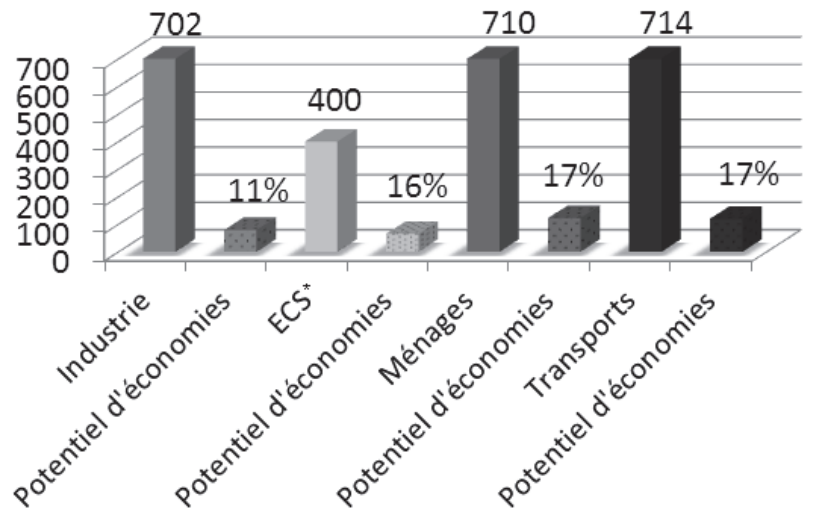

Sources: ministère fédéral de l'Économie et de l'Énergie, données sur l'énergie 2012; scénario d'efficacité énergétique de l'étude EnEffVSys de la dena et de Frontier Economics, 2012. * ECS : échanges, commerce, services.

En matière d'efficacité énergétique, on cherche avant tout à exploiter des potentiels économiques. Le graphique 1 montre l'importance de ces potentiels dans

\footnotetext{
${ }^{2}$ Depuis le $1^{\text {er }}$ janvier 2013, les entreprises concernées doivent justifier de la mise en œuvre d'un système de management de l'énergie répondant à des normes précises pour pouvoir bénéficier de cet allègement fiscal, $\mathrm{NdE}$.
} 
les différents secteurs de consommation. On constate qu'il existe des potentiels économiques d'efficacité énergétique dans tous les secteurs. Pour les exploiter, il faut surmonter divers obstacles :

- les ménages, les entreprises et les communes ne savent pas précisément combien d'énergie ils consomment, ni comment ils pourraient réduire leur consommation d'énergie et le prix qu'ils paient ;

- la tâche semble trop complexe, fragmentaire et coûteuse ;

- l'offre de produits et de services semble trop floue.

Associer l'information à l'incitation

Pour ces raisons, en plus de l'incitation, le NAPE mise largement sur l'information et la responsabilisation, par exemple au moyen de réseaux, de campagnes d'information, d'étiquetages, de normes de qualité, de conseils. La dena est fortement impliquée dans les campagnes gouvernementales. Aucun gouvernement ne peut se passer de ces campagnes, car à mon avis, il faut continuer à sensibiliser la population à la nécessité d'économiser l'énergie.

\section{Le financement dans le domaine des énergies renouvelables et des systèmes énergétiques}

\section{Déséquilibres de financement}

Chaque année, la loi allemande sur les énergies renouvelables (EEG) répartit 20 milliards d'euros pour le développement de ces énergies via la redevance EEG (EEG-Umlage). En comparaison, il faudra plus de 20 milliards pour développer le réseau électrique, mais sur une période de 10 ans. Le déséquilibre est également remarquable par rapport aux quelque 3 milliards d'euros consacrés chaque année à l'efficacité énergétique dans le budget de l'État.

Dans les années qui viennent, nous devons rééquilibrer la situation. En effet, le montant dévolu aux énergies renouvelables n'est pas disponible pour soutenir d'autres mesures. Pourtant, l'efficacité énergétique et les énergies renouvelables peuvent en principe apporter la même contribution à la transition énergétique. Nous devons mieux coordonner les instruments et, de mon point de vue, sortir un peu du surfinancement des énergies renouvelables. La réforme de la loi EEG adoptée en 2014 constitue un pas important dans cette direction. Nous devons maintenant voir si cela suffira.

\section{L'évolution du marché de l'électricité}

Le développement des énergies renouvelables a eu des effets très importants sur le système énergétique dans son ensemble, et en particulier sur le marché de l'électricité. Pour le gouvernement allemand, la question se pose désormais de 
savoir s'il est prêt à ouvrir un nouveau réservoir de subventions pour assurer l'approvisionnement du marché de l'électricité - le «marché de capacité » des centrales à énergie fossile - ou s'il va essayer d'éviter ce recours et va miser sur un autre concept. Le livre vert Ein Strommarkt für die Energiewende («Un marché de l'électricité pour le tournant énergétique ») publié en octobre 2014 montre qu'il s'oriente plutôt vers la deuxième piste. Par conséquent, le nouveau marché doit se développer correctement.

Comment ce développement se profile-t-il ? Actuellement, le marché de l'électricité en Allemagne est en train de subir une profonde mutation. En effet, alors qu'auparavant les centrales électriques suivaient la demande, selon la nouvelle conception du marché de l'électricité, ce sont les producteurs d'énergie flexibles autant que les consommateurs qui vont s'adapter aux fluctuations de l'offre, au moyen de transferts de charge, d'options de flexibilité et de solutions de stockage.

\section{Maîtrise de la demande (demand side management)}

La maîtrise de la demande ou demand side management, c'est-à-dire le pilotage flexible de la consommation dans les entreprises, est un instrument important pour rendre le marché de l'électricité plus flexible et permettre ainsi l'intégration des énergies renouvelables. De nombreuses études montrent par exemple que le potentiel technique de flexibilité pour le transfert de charge dans l'économie allemande est plus important que ce qui sera nécessaire pour la transition énergétique. La question n'est cependant pas de savoir ce qui est techniquement possible, mais ce qui, en pratique, est réalisable économiquement pour les entreprises en termes de transfert de charge.

La dena a deux projets pilotes, un en Bavière et un dans le Bade-Wurtemberg, dans le cadre desquels elle réalise des plans et des projets concrets avec des entreprises grosses consommatrices d'énergie. On cherche à savoir comment ces entreprises pourraient piloter leur production de manière à ce que leur consommation d'énergie s'adapte à l'offre des producteurs d'électricité. En bref, si les exploitants des réseaux de transport d'énergie ont besoin d'énergie d'équilibrage, ils peuvent actuellement en acheter auprès d'entreprises ayant des charges flexibles. C'est un report de charge, une gestion de la charge. La question est : peut-on ainsi renoncer à construire de nouvelles centrales électriques ? L'avenir nous le dira.

\section{La question du développement du réseau}

Une autre clé de la flexibilité du système électrique réside dans le développement du réseau. Ce sujet donne actuellement lieu à d'intenses discussions en Allemagne. On se demande si d'ici 2022 - lorsque les dernières centrales nucléaires seront mises à l'arrêt -, l'énergie éolienne en excédent dans le nord de l'Allemagne pourra être acheminée vers les centres de consommation dans le 
sud du pays. Nous nous trouvons face à un important défi, notamment en ce qui concerne l'acceptation du tournant énergétique par la population. En fin de compte, une bonne conception du marché de l'électricité ne suffira pas s'il n'y a pas de lignes électriques pour transporter l'énergie.

Si le développement du réseau n'est pas assez rapide, il faut se demander si nous pouvons maintenir notre zone de tarification unique, ou si, à cause des nombreuses interventions dans le fonctionnement des centrales, notamment sous la pression de Bruxelles, nous devrons passer à un système à deux zones, dont personne ne veut naturellement en Allemagne. La réponse à cette question n'est pas exclusivement entre des mains allemandes. Cela montre aussi combien tous les aspects de la transition énergétique sont interconnectés et combien il est difficile de mettre en œuvre un projet d'une telle envergure en aussi peu de temps, en particulier lorsque les projets d'infrastructure nécessitent l'approbation de la population dans les régions concernées.

LA TRANSITION ÉNERGÉTIQUE EST UN VASTE DÉFI. Jusqu'à présent, le financement s'est concentré sur les énergies renouvelables; il faut soutenir plus fortement l'efficacité énergétique. C'est la direction que prend le gouvernement fédéral avec ses nouveaux programmes. Du point de vue de la dena, il faut toujours garder un objectif en tête : assurer le pilotage souhaité au plan politique par un cadre clairement défini et fiable, et autoriser au sein de ce cadre autant de marché et d'ouverture technologique que possible. En résumé, il faut autant de marché que possible, autant de pilotage que nécessaire, en pensant à l'efficacité énergétique avant tout.

Traduction de Marie-Céline GEORG 
- III -

EXEMPLES DE COOPÉRATION FRANCO-ALLEMANDE 



\section{EXPOSÉ INTRODUCTIF}

\section{Le couple franco-allemand : moteur de la transition énergétique ?'}

\section{Jean-Claude PERRAUDIN}

Parmi les sujets de divergence entre les politiques française et allemande, l'énergie est généralement le plus fréquemment cité, qu'il s'agisse des modes de production ou de la vision stratégique à long terme.

Concernant les mix électriques, les différences se sont fortement creusées ces dernières années. Aujourd'hui, la production allemande se répartit entre $14 \%$ de nucléaire, $42 \%$ de lignite et de charbon et plus de $30 \%$ de renouvelables. En France, d'où les fossiles solides ont pratiquement disparu, le nucléaire contribue à plus de $75 \%$ et les renouvelables à $16 \%$, dont $12 \%$ d'hydraulique sur lequel l'Allemagne ne peut compter qu'à hauteur de 3 à $4 \%$.

À moyen terme, ces divergences devraient encore s'accentuer: en Allemagne, le nucléaire aura totalement disparu fin 2022 et le charbon ne devrait connaître qu'une décroissance lente, peu à peu remplacé par les énergies renouvelables épaulées dans une première phase par le gaz. En France, la diversification engagée prévoit de faire passer la part du nucléaire dans la production d'électricité à $50 \%$ à l'horizon 2025, qui continuera donc d'assurer la production de base et de garantir la sécurité d'approvisionnement.

Les visions pour préparer l'avenir énergétique à long terme présentent aussi bien des différences: en Allemagne, il faut parler de tournant (die Energiewende) tant les objectifs sont ambitieux, voire risqués selon certains, alors que la France s'est engagée dans une transition plus progressive, régie par la loi « transition énergétique pour une croissance verte » adoptée en août 2015, qui encadre avec rigueur le processus et définit les modalités de développement du mix.

Avec des stratégies et des trajectoires nationales aussi différentes, on peut légitimement s'interroger sur la capacité du couple franco-allemand à constituer un moteur pour la transition énergétique : tout semble plutôt indiquer que les deux axes tournent dans des sens opposés.

Si la réflexion porte sur la seule vision de la composition du mix à long terme, la réponse à la question posée en titre est logiquement négative. Mais il n'en est pas de même si l'on observe que les deux pays partagent une même ambition de décarbonation de leur économie, renforcée depuis décembre 2015 par le succès de la conférence environnementale de Paris (COP21), et par le

\footnotetext{
${ }^{1}$ Article rédigé en octobre $2016, \mathrm{NdE}$.
} 
souci de répondre à ses prometteuses conclusions. Les transitions qu'elles imposent, dont chaque pays doit assumer la responsabilité, devront en effet reposer sur des moyens de mise en œuvre communs, certes utilisés dans des proportions différentes conformément à leur vision nationale.

Parmi ces moyens, les nouvelles technologies occupent une place privilégiée. Au-delà des nécessaires réglementations et incitations, elles seules pourront en effet contribuer concrètement à assurer demain une production d'énergie à la fois fiable et moins émettrice de $\mathrm{CO}_{2}$, et à mettre en œuvre les nécessaires mesures de réduction de consommation, notamment en renforçant l'efficacité énergétique.

La plupart de ces technologies sont connues, mais beaucoup nécessitent un saut technologique urgent. Celui-ci ne se fera pas sans un effort considérable en matière de recherche et d'innovation. Les deux pays possèdent chacun dans ce domaine des atouts et des compétences uniques dont la complémentarité et la réunion pourraient constituer un socle commun permettant de réduire notablement les coûts et les délais de réalisation des indispensables nouveaux dispositifs de production, de gestion et de consommation durables de l'énergie.

LA SCIENCE EST UNIVERSELLE et ne connaît pas de frontières. S'il est un domaine autour duquel les deux pays peuvent se retrouver et où l'union peut faire la force, c'est bien la recherche et l'innovation en énergie qui pourraient sans nul doute constituer ce moteur franco-allemand que tous appellent de leur vœux et qui peine toujours à démarrer. 


\section{TANDEM : coopération franco-allemande pour la transition énergétique locale ${ }^{1}$}

\section{Anne TURFIN}

La COP21, conférence de l'ONU sur le climat qui s'est tenue en décembre 2015, a débouché sur l'accord de Paris, dont l'objectif déclaré est de limiter le réchauffement mondial " nettement en dessous de $2{ }^{\circ} \mathrm{C}$ ", et même de tendre vers $1,5^{\circ} \mathrm{C}$. La conférence de Paris sur le climat a constitué un moment historique pour les villes et les communes, qui sont de plus en plus reconnues par la communauté internationale comme des acteurs importants pour atteindre les objectifs climatiques. Le rôle des communes dans l'organisation de la transition énergétique est désormais apprécié au plan international.

En Allemagne comme en France, la motivation est forte pour mettre en œuvre cette transition énergétique. Les deux pays, qui constituent également le cœur géographique de l'Europe, ont une influence centrale sur la politique européenne de protection du climat. La prospérité économique y est jusqu'à présent fortement liée à l'émission de $\mathrm{CO}_{2}$. Si les deux pays pouvaient concevoir leur politique de protection du climat de manière à permettre une transition énergétique alliant prospérité et développement économique, cet exemple constituerait une meilleure incitation pour la communauté internationale qu'un traité contraignant. La coopération franco-allemande doit être présentée de manière offensive afin de pouvoir remplir cette fonction d'exemple. L'article de Franca Diechtl, de l'Agence allemande de l'énergie (Deutsche Energie-Agentur, dena), et de Severin Fischer, paru en mai 2015 et intitulé «Unter neuen Vorzeichen. Transformationsprozesse und Kooperationsmodelle in den deutsch-französischen Energiebeziehungen », en donne une illustration très parlante : «L'Allemagne et la France se sont efforcées à plusieurs reprises au cours des dernières années de mettre en auvre une coopération concrète et de se positionner comme l'avant-garde de l'Europe. Des réflexions et des décisions communes d'ordre énergétique sont devenues le point de mire des Conseils des ministres francoallemands organisés régulièrement $[\ldots]^{2}$. » En ce qui concerne la mise en œuvre de mesures concrètes de coopération, il reste beaucoup à faire. Cependant, il existe des amorces prometteuses de projets communs, comme l'Office franco-allemand pour les énergies renouvelables (OFAEnR, renommé Office franco-allemand pour la transition énergétique - OFATE - en avril 2016) ou l'établissement d'une plate-forme énergétique franco-allemande par l'Agence

\footnotetext{
${ }^{1}$ Article rédigé en décembre 2016, NdE.

${ }^{2}$ Franca Diechtl, Severin Fischer, «Unter neuen Vorzeichen. Transformationsprozesse und Kooperationsmodelle in den deutsch-französischen Energiebeziehungen », DGAPanalyse, n 5, mai 2015, p. 7 [version française : Franca Diechtl, Severin Fischer, «Sous de nouveaux auspices : processus de transition et modèles de coopération au sein des relations énergétiques franco-allemandes », Vision franco-allemande, $\mathrm{n}^{\circ} 26$, juin 2015, p. 13.].
} 
française de l'environnement et de la maîtrise de l'énergie (ADEME) et la Deutsche Energie-Agentur (dena). Ces deux projets de coopération s'adressent aux ministères, aux entreprises, aux associations industrielles, aux établissements de recherche dans le domaine des énergies renouvelables de chaque pays. Les villes et les communes ne sont pas suffisamment impliquées dans les projets ci-dessus. C'est d'autant plus étonnant que les communes et les Länder ont un rôle important à jouer dans la mise en œuvre de la transition énergétique en Allemagne.

En mars 2013, les réseaux de villes Klima-Bündnis et Energy Cities, ainsi que l'Agence de l'énergie et du climat du Bade-Wurtemberg (Klimaschutzund Energieagentur Baden-Württemberg $\mathrm{GmbH}, \mathrm{KEA}$ ) ont organisé une réunion de deux jours rassemblant des communes françaises et allemandes à Stuttgart. Initialement prévue comme une rencontre des deux clubs de la Convention des maires, cette manifestation a donné lieu à un dialogue vivant non seulement entre des conseillers municipaux allemands et français, mais aussi avec des responsables communaux de l'énergie, des représentants des services communaux d'approvisionnement énergétique, des responsables économiques, des citoyens et des chercheurs. Elle a contribué à une meilleure compréhension de la situation de départ et des tâches à accomplir en matière de politique énergétique et climatique de part et d'autre du Rhin. On a pu constater que les objectifs et les obstacles, mais aussi les stratégies de résolution, n'étaient pas si différents : le souhait a été émis d'affronter ensemble ces défis et de rechercher des solutions dans le cadre d'un échange d'expérience toujours plus approfondi et de projets de coopération. C'est ainsi qu'est né le projet financé par l'Office fédéral de l'environnement (Umweltbundesamt) et l'ADEME française, «Energiewende et transition énergétique locale : partenariats franco-allemands pour le climat », auquel on a donné le judicieux nom de TANDEM.

\section{Le projet TANDEM}

Le projet TANDEM est animé conjointement par les réseaux Klima-Bündnis et Energy Cities, avec la participation de l'Agence de l'énergie et du climat du Bade-Wurtemberg (KEA) et de l'Institut franco-allemand (Deutsch-Französisches Institut, dfi).

Ce projet réunit des communes et des acteurs pertinents pour organiser et favoriser la protection du climat à l'échelon communal, afin de stimuler et d'organiser au niveau local, par un échange d'expérience approfondi et des projets de coopération, l'Energiewende allemande et la transition énergétique française.

L'objectif est d'accélérer la mise en œuvre des concepts communaux pour la protection du climat (kommunale Klimaschutzkonzepte) du côté allemand et des Plans climat-énergie territoriaux (PCET) du côté français. À partir des suggestions émises lors de la conférence organisée par Klima-Bündnis et 
Energy Cities sur le thème «Transition énergétique vs Energiewende » en mars 2013 à Stuttgart, des communes françaises et allemandes doivent former des partenariats pour la protection du climat. Dans ce cadre, des projets communs seront élaborés et mis en œuvre, avec les prestations et les investissements correspondants.

Pour créer une compréhension mutuelle, on a tout d'abord réalisé une analyse de la situation des deux pays en matière de protection communale du climat. À l'occasion des recherches menées pour cette analyse, on a réalisé une première série de fiches thématiques bilingues publiées sur Internet (http://villetandem.eu/ressources/fiches-thematiques.html ${ }^{3}$ ). Ces fiches traitent des thèmes suivants :

- projets scolaires ;

- dispositifs d'aides aux politiques climat-énergie ;

- rénovation thermique des bâtiments.

Les informations sont présentées dans des fiches bilingues de deux à quatre pages. Elles servent de base pour les webinaires ou constituent le résultat d'un événement de ce type.

Le format du webinaire s'est avéré particulièrement adapté pour transmettre des informations sur des thèmes spécifiques; c'est un moyen de communication facile à mettre en œuvre et peu onéreux pour approfondir les bases communes. Les webinaires se sont également révélés très utiles pour stimuler les partenariats. C'est pour cette raison que nous avons réalisé cinq webinaires sur les thèmes suivants (dont la moitié avec interprétation consécutive) :

- fonctionnement de l'administration française : le rôle des communes (mai 2015) ;

- fonctionnement de l'administration allemande : le rôle des communes (juin 2015) ;

- Masterplan $100 \%$ Klimaschutz (Plan $100 \%$ climat) et PCAET (Plan climat-air-énergie territorial): quelles possibilités de partenariat entre collectivités françaises et allemandes ? (juin 2015);

- Le couple TANDEM Épernay - Ettlingen partage son expérience (octobre 2015) ;

- Cit'ergie/eea [European Energy Award]: regards croisés francoallemands (juin 2016).

Une rubrique «Webinaires » est proposée sur le site Internet de TANDEM : http://ville-tandem.eu/ressources/webinaires.html ${ }^{4}$. On peut y revoir les webinaires passés qui y sont sauvegardés.

\footnotetext{
${ }^{3}$ Consulté le 10/03/2017.
}

${ }^{4}$ Consulté le 10/03/2017. 
À partir de là, des projets de coopération entre des communes allemandes et françaises ont été lancés, après une manifestation inaugurale organisée en France (Strasbourg, 2014).

\section{Chances et défis pour la création de partenariats franco-allemands de protection du climat}

Lorsqu'une ville allemande et une ville française s'engagent dans un partenariat de protection du climat, elles identifient rapidement les possibilités que cette coopération leur ouvre. Le partenariat y gagne le plus souvent une nouvelle dynamique, de nouvelles idées (communes) et des impulsions pour la protection du climat. C'est un moyen très simple de regarder la situation au-delà des frontières nationales et d'entrer en contact.

Cependant, réunir deux communes dans un partenariat pour la protection du climat est un processus de longue haleine. En règle générale, les coopérations transfrontalières ne sont pas à l'ordre du jour dans les services et les administrations chargés de l'énergie et du climat. Le manque de ressources humaines ou de soutien politique au sein de la commune, et parfois simplement le manque d'intérêt envers le partenariat ont été des obstacles fréquents à la création de ces partenariats. De plus, du côté français, les compétences en matière d'énergie et de protection du climat sont souvent aux mains des communautés de communes, tandis que les jumelages historiques concernent les villes, un obstacle supplémentaire qu'il a fallu prendre en compte.

Après le premier échange, les villes constatent qu'il existe de nombreuses convergences entre le Plan climat-air-énergie territorial (PCAET) et le Klimaschutzkonzept, et qu'elles ont beaucoup à apprendre les unes des autres. Ensemble, elles commencent à élaborer et mettre en œuvre des plans ambitieux de protection du climat (avec de bonnes perspectives de subventions). Cela donne aux citoyens une image positive de l'Europe et des relations franco-allemandes. À long terme, les partenariats TANDEM pourraient devenir un moteur du renforcement de la politique européenne. Le projet TANDEM s'est avéré très enrichissant pour tous les participants, non seulement au plan technique, mais aussi au plan humain.

\section{Constitution d'un partenariat TANDEM}

L'accompagnement de villes françaises et allemandes a montré que la construction d'un partenariat pour la protection du climat prenait du temps. Mais par quelles étapes faut-il passer pour qu'une ville française et une ville allemande constituent un partenariat TANDEM ?

La première étape est la recherche d'un partenaire. La plupart des villes décident de s'adresser à leur ville jumelée soit directement, soit, le plus souvent, via l'équipe TANDEM. En l'absence d'intérêt pour un tel projet, ou s'il manque des moyens, l'équipe TANDEM propose de chercher une ville parte- 
naire adaptée. La recherche commence par l'établissement d'un profil de la ville, qui est en quelque sorte sa carte de visite. Il contient les informations suivantes sous une forme claire et concise : un portrait de la ville, en signalant si elle est signataire de la Convention des maires ou certifiée European Energy Award, une description de la politique de protection du climat et de ses objectifs en la matière, un résumé du Klimaschutzkonzept (le cas échéant), les étapes/principaux projets de protection du climat en cours et des propositions de thématiques ou de projets pour la coopération. En tant que réseaux actifs de villes européennes en matière de protection du climat, Energy Cities en France et Klima-Bündnis en Allemagne disposent de contacts personnels avec de nombreux intéressés potentiels.

La deuxième étape consiste à mieux se connaître. Pour commencer, on échange des documents pertinents sur le sujet, en général de courtes présentations de projets de protection du climat. Dès qu'on a trouvé un partenaire intéressé, la première téléconférence peut avoir lieu. L'accent est mis sur la prise de contact personnelle et sur la recherche d'intérêts communs et de thèmes de coopération potentiels. Il s'est avéré judicieux d'organiser régulièrement des téléconférences pour entretenir le contact entre les villes partenaires, afin d'approfondir les thèmes de coopération possibles pendant la phase initiale et de promouvoir ensuite la mise en œuvre de projets.

La troisième étape est la rencontre sur place. Les responsables politiques, les services de l'environnement ou autres bureaux et responsables impliqués dans la protection du climat, ainsi que les entreprises communales (agences de l'énergie et régies municipales par exemple), consacrent une à deux journées à échanger sur la politique de protection du climat dans leurs collectivités territoriales respectives. On a constaté que l'association d'une présentation de projets environnementaux réussis et d'une visite donnait de bons résultats en termes d'inspiration mutuelle et d'unités de discussion/réflexion. Les rencontres sur place offrent les meilleures conditions pour discuter des thèmes de coopération et des projets.

La poursuite de la coopération entre les deux villes s'effectue par le biais de conférences téléphoniques et d'autres rencontres organisées sur place. Une rencontre par an est un minimum; il n'y a aucune limite supérieure, c'est avant tout une question de distance. En raison des changements de personnel, il peut arriver que certaines villes choisissent de sortir du processus de coopération, ou de le mettre en sommeil jusqu'à ce que les conditions redeviennent favorables.

Fin novembre 2016, on comptait déjà dix couples TANDEM. Quatre autres se trouvent encore au stade de la prise de décision, et plusieurs communes sont à la recherche d'un partenaire.

À l'exception d'un couple TANDEM, tous les partenariats se sont créés sur la base de jumelages existants et ont donné lieu à au moins une rencontre de coopération (Ettlingen/Épernay, Herten/Arras, Fribourg-en-Brisgau/Besançon, 
Essen/Grenoble, Nuremberg/Nice, Velbert/Châtellerault, Karlsruhe/Nancy, Stuttgart/Strasbourg, Worms/Metz, Göttingen/Pau).

\section{Carte 1 : Couples TANDEM - Présentation de partenariats en cours}

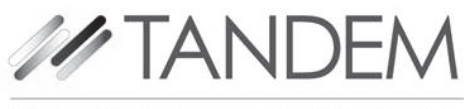

DEUTSCH-FRANZOOSISCHE KLMAPARTINERSCHATEN FÜR DIE LOKALF ENERGIEWENDE COOPÉRATON Franco-ALIEMANDE POUR LA TRANSTION ÉNERGÉticue Locale

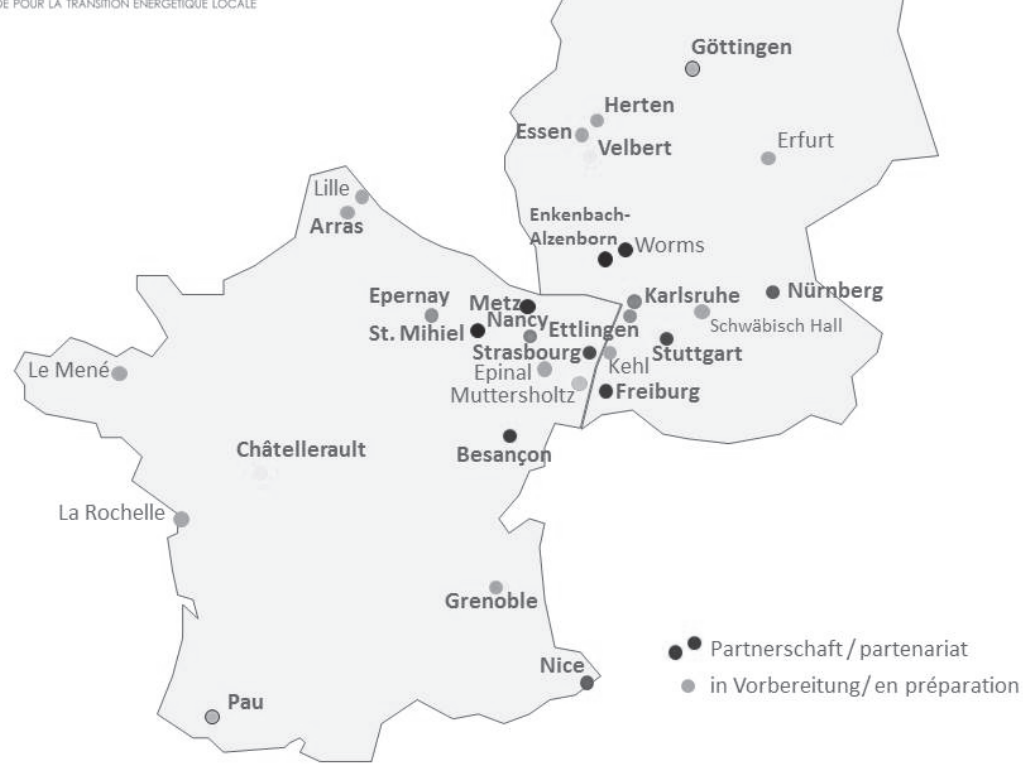

Source : http://ville-tandem.eu/couples-tandem.html [consulté le 13/03/2017].

Il s'est avéré que trois modes de coopération sont au cœur d'un partenariat pour la protection du climat :

- l'échange d'expérience - quand deux villes souhaitent mettre en œuvre des projets similaires ;

- le transfert de connaissances - quand une ville souhaite mettre en œuvre un projet qui existe déjà dans sa commune partenaire ;

- les projets communs - dans la plupart des cas, il s'agit de projets qui s'adressent aux citoyens, mais il peut aussi s'agir de mesures d'investissement.

Un bon exemple d'échange d'expérience est le projet de travaux de rénovation à Herten et Arras. Ces deux villes sont en train de planifier en parallèle et en concertation deux chantiers de rénovation énergétique des équipements culturels publics. 
En ce qui concerne la mise en œuvre commune d'un projet, on peut citer l'achat prévu d'un espace info énergie mobile par les villes d'Épernay et d'Ettlingen.

Quant à Besançon et Fribourg-en-Brisgau, elles ont réalisé un passionnant projet de transfert de connaissances : le projet «Familles à énergie positive » de Besançon a été adapté à la situation allemande par la ville de Fribourg-enBrisgau et mis en œuvre sous le nom de « 200 Familien aktiv fürs Klima» (200 familles actives pour le climat). Dans le cadre de ce projet, les citoyens participants ont été accompagnés pour organiser leur mode de vie de manière plus durable et économe en ressources.

Le projet TANDEM a donné naissance à de très intéressantes coopérations. Il a également permis une collaboration constructive entre les clubs français et allemands de la Convention des maires. Ceux-ci ont élaboré une déclaration commune, «Transition énergétique au niveau local et Energiewende - Recommandations aux instances nationales de France et d'Allemagne pour accélérer la transition énergétique au niveau local », qu'ils ont adoptée à l'occasion du deuxième colloque TANDEM. Le $1^{\text {er }}$ mars 2016 , des représentants du club allemand ont présenté cette déclaration commune à la ministre fédérale de l'Environnement, Barbara Hendricks, lors de la séance plénière de la $9^{\mathrm{e}}$ conférence du Deutscher Städte- und Gemeindebund (Association des villes et municipalités allemandes) sur la protection du climat, «Kommunen aktiv für den Klimaschutz ».

EN REVENANT SUR LES TROIS DERNIÈRES ANNÉES, on peut dire que le projet TANDEM est un franc succès. L'implication de villes dans un projet francoallemand sur le thème de la transition énergétique comble une lacune de la coopération entre les deux pays et offre des perspectives très prometteuses. En effet, une majorité de la population européenne vit en ville. Les villes sont responsables d'environ $80 \%$ des émissions de gaz à effet de serre, mais ce sont aussi des acteurs importants de la transition énergétique, qui peuvent mettre en œuvre les mesures de protection du climat bien plus rapidement que les États. Néanmoins, il faudra encore de nombreux partenariats TANDEM pour relever les défis de la transition énergétique et du changement climatique.

Traduction de Marie-Céline GEORG 



\section{Protection du climat, énergies renouvelables et efficacité énergétique dans la Région métropolitaine trinationale du Rhin supérieur ${ }^{1}$}

\section{Jürgen OSER}

La Région métropolitaine trinationale du Rhin supérieur (RMT) englobe l'espace transfrontalier franco-germano-suisse constitué par quatre territoires : l'Alsace, la Suisse du NordOuest, le sud du Palatinat et une partie du Pays de Bade. Elle compte six millions d'habitants sur une superficie de 21517 km² $^{2}$ Délimitée par la Forêt-Noire à l'est, les Vosges à l'ouest et une partie du Jura au sud, elle est largement couverte de forêts - au total, 44 \% du territoire du Rhin supérieur. Dans le piémont, on cultive la vigne. L'agriculture occupe $41 \%$ du territoire, tandis que $2 \%$ sont constitués de cours d'eau et d'autres espaces non exploitables. Les $13 \%$ restants sont dévolus aux agglomérations, à l'industrie et aux voies de communication. Le produit intérieur brut (PIB) du Rhin supérieur s'élève à 240 milliards d'euros par an (2014).

Instituée par l'accord de Bonn le 22 octobre 1975, la Commission intergouvernementale formée de représentants des trois États a constitué la base d'une approche systématique dans les contacts transfrontaliers.

La création de la Conférence franco-germano-suisse du Rhin supérieur (CRS) en 1991 représente la conséquence logique du développement souhaité par ses initiateurs, y compris à l'échelle régionale.

Le secrétariat commun de la CRS, créé en 1996 à Kehl avec un secrétaire de délégation de chacun des trois États, joue un rôle de charnière tout en garantissant le lien direct des autorités entre elles.

Plus de 500 experts participent aujourd'hui aux différents groupes de travail : Éducation et formation, Sport, Entraide en cas de catastrophe, Jeunesse, Santé, Environnement, Culture, Aménagement du territoire, Agriculture, Économie, Climat et énergie, Transports.

Seule une étroite coopération permet de relever la plupart des défis posés, qui vont de la protection de l'environnement à la politique de l'emploi, en passant par le financement des infrastructures. Les gens doivent ressentir l'utilité de la coopération transfrontalière pour que l'espace vital et économique commun du Rhin supérieur puisse se concrétiser. Ces 25 dernières années, plus de 500 projets ont pu être réalisés.

La gouvernance de la Région métropolitaine trinationale du Rhin supérieur

La politique seule ne peut pas aborder tous les sujets. Pour mieux exploiter nos forces communes, des représentants de l'Allemagne, de la France et de la Suisse ont signé le 10 décembre 2010 la déclaration d'Offenburg créant la Région métropolitaine trinationale du Rhin supérieur (RMT). Celle-ci s'appuie sur quatre

\footnotetext{
${ }^{1}$ Article rédigé en octobre $2016, \mathrm{NdE}$.
} 
piliers : les sciences, l'économie, la société civile et la politique. Dans le cadre d'une gouvernance à plusieurs niveaux, les acteurs travaillent ensemble au développement de cet espace transfrontalier.

La stratégie 2020 de la RMT, «Agir et se développer ensemble », définit les 10 principaux objectifs de cette région à l'échelle transfrontalière. Ceux-ci s'inspirent de la nouvelle stratégie européenne Europe 2020, qui vise à développer l'emploi, à assurer une croissance intelligente, durable et inclusive, et à mieux coordonner les politiques nationales et européennes d'une part, et la Nouvelle politique régionale suisse d'autre part.

Ces objectifs figurent dans le programme opérationnel INTERREG V A Rhin supérieur, qui représente, pour la période 2014-2020, la plus importante source de financement pour le développement régional, avec une dotation de 109 millions d'euros.

Le thème de l'énergie y joue un rôle important. L'enjeu à long terme est essentiellement de garantir l'approvisionnement énergétique du Rhin supérieur, en créant un pôle d'excellence pour les énergies renouvelables et l'efficacité énergétique. Pour cela, il s'agit de renforcer la coopération en matière de production, d'économies, de stockage et d'approvisionnement, ainsi que la coordination trinationale et l'échange d'informations sur l'énergie nucléaire à travers la Commission locale d'information et de surveillance (CLIS).

\section{La commission Climat et Énergie et la plate-forme d'action TRION-climate ${ }^{2}$}

Voici plusieurs années déjà que la corporation Sanitär/Heizung/Klima (SHK) de Kehl - Pays de Hanau/Lahr est en contact avec la Corporation des professionnels ferblantiers installateurs (COPFI), son homologue dans le département du Bas-Rhin. S'agissant de la mise en œuvre de nouvelles technologies pour lutter contre le changement climatique, le défi à relever est le même de part et d'autre du Rhin. Mais en France comme en Allemagne, les responsables n'entendaient pas se contenter de proposer des rencontres et de promouvoir l'apprentissage transfrontalier.

Avec le soutien de l'association kehloise TRION-climate, de l'Ortenauer Energieagentur et du centre de ressources energivie.pro à Strasbourg, ils ont organisé en avril 2015 un premier atelier transfrontalier sur le thème de la ventilation double flux destiné aux membres des deux corporations. Des experts alsaciens et badois ont pris la parole sur les aspects techniques mais aussi, notamment, sur les aspects juridiques en France et en Allemagne.

Les responsables de la Conférence du Rhin supérieur se sont penchés sur les questions climatiques et énergétiques bien avant la catastrophe de Fukushima : le changement climatique ne s'arrête pas aux frontières et, dans l'espace trinational, on n'a pas tardé à se rendre compte que la lutte contre le réchauffement

\footnotetext{
${ }^{2}$ Reinhard Reck, 1975-2015 - 40 ans de coopération transfrontalière dans le Rhin supérieur, Conférence franco-germano-suisse du Rhin supérieur, Kehl, 2015.
} 
de la planète exigeait des initiatives communes. De même, on s'efforce depuis longtemps de coopérer pour promouvoir les énergies renouvelables. Les acteurs du Rhin supérieur affichent l'objectif ambitieux de faire de leur région un modèle en matière climatique et énergétique.

En 2009, avec la création à Kehl du réseau TRION pour l'énergie et le climat (un projet porté par le Regierungspräsidium de Fribourg-en-Brisgau), la Conférence du Rhin supérieur a jeté les bases d'une approche trinationale en matière de lutte contre le changement climatique et fait figure de pionnier à l'échelle européenne. Depuis septembre 2015, l'ancien réseau TRION a cédé la place à l'association TRION-climate, une structure formelle dotée de la personnalité juridique.

Son bureau compte aujourd'hui quatre collaborateurs. Ses tâches principales sont l'échange transfrontalier d'informations, la promotion de la coopération entre représentants des milieux scientifiques, économiques et politiques sur des projets concrets, et enfin la collecte de données dans la région frontalière. Ainsi, les acteurs disposent aujourd'hui d'une image précise des besoins en énergie et des potentiels d'économies pour le Rhin supérieur. TRION est particulièrement engagé dans l'organisation de salons professionnels et de manifestations d'information (comme les green visits).

La commission Climat et Énergie de la Conférence du Rhin supérieur s'efforce d'aborder les sujets relevant de sa compétence selon une approche interdisciplinaire. Aussi intègre-t-elle aussi bien des experts du climat que des spécialistes des politiques de l'environnement et des transports, ou encore des représentants de l'agriculture et de la sylviculture.

La première Stratégie transfrontalière sur le climat et l'énergie a été adoptée en 2006, puis mise à jour en juin 2013. Signée par des représentants des trois pays partenaires, elle souligne d'emblée que le Rhin supérieur joue un rôle précurseur au niveau européen dans le domaine de la protection du climat et de l'énergie. Un de ses objectifs est de réduire les émissions de gaz à effet de serre d'au moins $80 \%$ par rapport à 2000 d'ici 2050. En outre, à compter de 2050, dans l'ensemble du Rhin supérieur, les besoins en énergie pour l'électricité et la production de chaleur doivent pouvoir être couverts le plus largement et durablement possible par des sources d'énergies renouvelables.

\section{Stratégie transfrontalière sur le climat et l'énergie pour la Région métropolitaine trinationale du Rhin supérieur \\ Comité directeur de la Conférence du Rhin supérieur du 28 juin 2013}

\section{Objectif :}

Le Rhin supérieur joue un rôle précurseur au niveau européen dans le domaine de la protection du climat et de l'énergie en renforçant et en cultivant son image marquée par l'innovation et le bon sens écologique et économique.

Stratégie:

1. Les futurs défis auxquels sera confronté le Rhin supérieur dans le secteur de la protection du climat et de l'énergie doivent être abordés conjointement, dans le cadre d'une approche interdis- 
ciplinaire et transfrontalière. L'accent sera mis plus particulièrement sur une coopération des principaux acteurs au niveau politique, administratif, communal, environnemental, économique et scientifique, de même que sur l'intégration des initiatives publiques et privées en cours.

2. Les stratégies et lois de I'Union européenne (UE), de la France, de l'Allemagne, de la Suisse et des régions formant le Rhin supérieur prévalent face à la présente stratégie.

3. II importe que les objectifs politiques en matière de climat et d'énergie de la présente stratégie concordent avec les objectifs environnementaux, économiques et sociétaux, dans le sens d'un développement durable.

4. D'ici 2050, dans l'ensemble du Rhin supérieur, les émissions de gaz à effet de serre doivent être réduites de manière continue et durable d'au moins $80 \%$ par rapport à $2000^{3}$.

5. D'ici 2050, dans l'ensemble du Rhin supérieur, la consommation d'énergie primaire devra baisser durablement d'au moins $35 \%$ par rapport à $2000^{4}$.

6. À partir de 2021, tous les bâtiments neufs de l'UE devront être énergétiquement pratiquement autonomes pour le chauffage, l'eau chaude, la ventilation et la climatisation. Les bâtiments publics neufs devront remplir cette exigence dès 2019. Le besoin résiduel d'énergie devrait provenir autant que possible d'énergies renouvelables produites directement sur place ou à proximité du bâtiment.

La rénovation énergétique du parc bâti doit reposer sur l'objectif de réduire le besoin spécifique moyen en chauffage de $130 \mathrm{kWh} / \mathrm{m}^{2}$ par an à $35 \mathrm{kWh} / \mathrm{m}^{2}$ par an $\mathrm{n}^{5}$ 'ici 2050. Le taux de rénovation doit atteindre au moins $2 \%$ par an.

7. À compter de 2050, dans l'ensemble du Rhin supérieur, les besoins en énergie pour l'électricité et la production de chaleur doivent pouvoir être couverts le plus largement et durablement possible par des sources d'énergies renouvelables, en assurant à tout moment la sécurité d'approvisionnement notamment par l'extension des réseaux. L'atteinte de cet objectif exige l'utilisation de toutes les sources d'énergies renouvelables présentes dans le Rhin supérieur.

8. Les membres de la Conférence du Rhin supérieur (la France, l'Allemagne et la Suisse) chargent la commission Climat et Énergie de la mise en œuvre stratégique de la présente stratégie et TRION, le réseau Énergie-Climat de la Région métropolitaine du Rhin supérieur, de la mise en œuvre opérationnelle des différentes mesures et projets dont l'accent sera mis sur les aspects suivants :

a) coordination transfrontalière des agences régionales sur le climat/énergie et les clusters ;

b) mise en réseau des acteurs issus des domaines de l'environnement, de l'économie, des sciences et autres acteurs ;

c) organisation d'échanges transfrontaliers des savoirs, des formations et des expériences ;

d) compilation de données liées à l'énergie et estimation de potentiels, pour optimiser la mise en œuvre des actions, évaluer les résultats et soutenir la prise de décisions stratégiques ;

e) comparabilité transfrontalière de standards, de labels et d'autres données techniques ;

f) identification, soutien et mise en œuvre de projets exemplaires communs et développement de projets européens d'innovation ;

g) soutien à la mise sur le marché notamment de projets et technologies innovants.

\section{Utilisation concrète des énergies renouvelables dans le Rhin supérieur ${ }^{6}$}

La force hydraulique du Rhin est depuis longtemps utilisée par les États riverains pour produire de l'énergie. Dans le traité de Versailles, en 1919, la France

\footnotetext{
${ }^{3} 41$ millions de tonnes selon le rapport Énergie et protection du climat dans le Rhin supérieur de mars 2006.

${ }^{4} 215$ TWh selon le rapport Énergie et protection du climat dans le Rhin supérieur de mars 2006.

${ }^{5}$ Selon les indications dans la directive européenne 2010/31/EU.

${ }^{6}$ Centre régional de documentation pédagogique d'Alsace, en collaboration avec le Landesmedienzentrum Baden-Württemberg et le Landesmedienzentrum Rheinland-Pfalz, Vivre dans le Rhin supérieur - Manuel pour une Europe sans frontières; Groupe de travail Éducation et formation de la CRS.
} 
avait obtenu le droit d'utiliser la section frontalière du Rhin pour la production d'électricité et la navigation.

Entre 1932 et 1959, la France a construit un canal latéral équipé de quatre centrales hydroélectriques et écluses sur la rive gauche du Rhin entre Bâle et Breisach (Allemagne). Quatre centrales «en feston»seront ensuite construites conjointement par la France et l'Allemagne entre Breisach et Strasbourg (convention de 1956). Ces dérivations réalisées dans la zone partiellement canalisée ont également une utilité écologique, puisqu'après chaque centrale, l'eau est renvoyée dans le lit Vieux Rhin pour y maintenir un certain niveau d'eau.

Les centrales hydroélectriques implantées en aval de Strasbourg sont des aménagements franco-allemands (convention de 1969) «en ligne », directement sur le lit du fleuve.

Il existe aussi des barrages transfrontaliers entre l'Allemagne et la Suisse. Au total, on a ainsi 22 centrales hydroélectriques en fonctionnement le long du Rhin, qui couvrent jusqu'à un tiers de la consommation d'énergie du Rhin supérieur.

\section{Géothermie}

Le graben du Rhin supérieur est une région particulièrement intéressante pour l'exploitation géothermique. En Alsace, le site de Soultz-sous-Forêts se distingue par une hausse très rapide de la température du sous-sol en fonction de la profondeur de forage. L'eau circulant dans l'échangeur de chaleur géologique est pompée à une température pouvant atteindre $175^{\circ} \mathrm{C}$ et utilisée dans une centrale à technologie spéciale (Organic Rankine Cycle, ORC) pour fabriquer de l'électricité, puis, une fois refroidie, réinjectée dans le forage.

C'est ainsi qu'un projet de recherche international, financé par l'Union européenne, la France, l'Allemagne, ainsi que le Royaume-Uni dans sa phase initiale, a abouti à un succès. Des entreprises énergétiques françaises et allemandes ainsi que des équipes de chercheurs d'autres États y ont activement participé ${ }^{7}$.

Un projet similaire de géothermie hydrothermale est en cours à Rittershoffen (France). Dans une zone de faille géologique, de l'eau thermale à une température de 150 à $170{ }^{\circ} \mathrm{C}$ est captée grâce à un forage de 2500 à $3000 \mathrm{~m}$ de profondeur. Le circuit du forage permet de pomper l'eau chaude vers la surface pour en exploiter l'énergie. Un second puits (doublet) sert à réinjecter l'eau refroidie dans la roche. Un système de conduites achemine l'énergie vers l'amidonnerie Roquette située à $15 \mathrm{~km}$ de là, au bord du Rhin. La centrale produit 190 millions de $\mathrm{kWh}$ d'énergie thermique par an, ce qui couvre un quart des besoins en chaleur de process (16 000 tonnes équivalent pétrole) de l'usine.

\footnotetext{
${ }^{7}$ Service d'information BINE de l'Institut Leibniz pour les infrastructures d'information (FIZ Karlsruhe).
} 


\section{Risques de la géothermie ${ }^{8}$}

En décembre 2006, un forage géothermique test d'une profondeur de $5000 \mathrm{~m}$ a été réalisé à Bâle. On a ensuite injecté de l'eau dans la roche afin de la fracturer et d'améliorer sa perméabilité.

L'injection d'eau sous pression a provoqué en décembre 2006 des séismes perceptibles atteignant 3,5 de magnitude. Les secousses se sont poursuivies jusqu'en février 2007. Elles n'ont pas fait de dégâts notables, mais la population a été perturbée. Après ces événements, la société Geopower Basel AG a démonté la tour de forage et stoppé le projet.

Des forages géothermiques à faible profondeur $(130 \mathrm{~m})$ destinés au chauffage de l'hôtel de ville rénové à Staufen (Allemagne) sont responsables de soulèvements de terrain dans la ville. On avait foré dans la nappe phréatique sous pression, ce qui a permis à l'eau de remonter dans des couches géologiques (anhydrite) susceptibles de gonfler en présence de liquide. En octobre 2010, on recensait 268 maisons endommagées, dont 127 sérieusement. Les dernières mesures montrent que le soulèvement, qui a débuté il y a plus de sept ans, se poursuit encore au centre de la ville à un rythme de 2,6 à 2,8 $\mathrm{mm}$ par mois. Des problèmes similaires se sont produits en Alsace, dans le village de Lochwiller, toutefois sans atteindre la même ampleur.

\section{Utilisation de l'énergie éolienne ${ }^{9}$}

Aujourd'hui déjà, les énergies renouvelables assurent plus de $23 \%$ de la production d'électricité dans le Bade-Wurtemberg. D'ici 2020, ce pourcentage doit passer à $38 \%$, pour atteindre $80 \%$ d'ici 2050 .

C'est pourquoi le gouvernement du Land a jeté les bases d'un développement systématique de l'éolien en modifiant la loi sur la planification du territoire, en établissant des centres de compétences dans les quatre Regierungspräsidien, et en publiant un décret sur l'énergie éolienne ainsi que d'autres informations. Il soutient en permanence ce développement par des lignes directrices, des expertises, des guides et des conseils d'évaluation.

Parmi les énergies renouvelables, l'éolien terrestre (ou onshore) constitue la technologie la plus économique pour fournir de l'électricité. Peu encombrant, il offre également aux citoyens de bonnes possibilités de participer activement à la transition énergétique sous forme de «projets éoliens citoyens » en investissant et en percevant directement des bénéfices.

En août 2015, on comptait 400 éoliennes en fonctionnement dans tout le Land, 121 étaient en construction, et 279 avaient déjà obtenu ou au moins demandé une autorisation - dont 99 demandes et 58 autorisations rien que pour 2015.

\footnotetext{
${ }^{8}$ Ralf Deckert, Stuttgarter Nachrichten, 9 mars 2015.

${ }^{9}$ TRION-climate.
} 
Une éolienne récemment construite sur le sommet badois du Hornisgrinde (1163 m d'altitude), à l'est de Strasbourg, remplace trois installations plus petites datant de 1994. D'une hauteur totale de $120 \mathrm{~m}$, elle délivre une puissance de 2,3 MW, contre environ $350 \mathrm{~kW}$ au total et seulement $35 \mathrm{~m}$ de haut pour les trois précédentes. La nouvelle éolienne fournit ainsi pratiquement sept fois plus d'électricité.

Le grand nombre d'éoliennes offshore le long des côtes de la mer du Nord et de la Baltique conduit actuellement à planifier et construire de nouvelles lignes électriques à $380 \mathrm{kV}$ du nord vers le sud de l'Allemagne.

Les premières éoliennes alsaciennes sont implantées à Dehlingen, à la limite de la Lorraine. Ce projet a été porté par les 32 communes de la Communauté de communes de l'Alsace bossue $(\mathrm{CCAB})$ et mené à bien en février 2013. Les cinq éoliennes, dont les mâts atteignent $80 \mathrm{~m}$ pour une envergure de pales de $45 \mathrm{~m}$ et un poids de 70 tonnes, ont été construites par le groupe Nordex. Le parc possède une puissance totale de 12,5 MW.

\section{Photovoltaïque}

Les installations photovoltaïques convertissent la lumière du Soleil en électricité. Soit on l'utilise directement sur le lieu de production, soit on la réinjecte dans le réseau, par exemple le réseau communal en Allemagne. En contrepartie, le propriétaire reçoit une rémunération de rachat. La loi allemande sur la chaleur d'origine renouvelable (Erneuerbare-Energien-Wärmegesetz) impose un quota d'énergies renouvelables pour la production de la chaleur nécessaire aux bâtiments neufs. Cependant, le photovoltaïque n'est pas obligatoire.

En Alsace aussi, on voit de plus en plus de grandes installations photovoltaïques, en particulier à la campagne. Les petites installations sont de plus en plus fréquentes sur les toits des maisons individuelles. Le courant est racheté par Électricité de France (EDF) ou Électricité de Strasbourg. Les panneaux photovoltaïques sont souvent associés à des pompes à chaleur air-air électriques.

\section{Utilisation de la biomasse dans le Rhin supérieur}

Le projet trinational OUI Biomasse - Innovations pour une utilisation durable de la biomasse dans la région du Rhin supérieur - a pris fin en juin 2015 après trois ans d'existence. À l'issue de cette période, un consortium d'universités et d'instituts de recherche a présenté un guide pour une utilisation durable de la biomasse dans la région du Rhin supérieur.

Ce projet était financé par le programme européen INTERREG IV A avec un cofinancement par l'Offensive Sciences de la RMT (Région métropolitaine trinationale du Rhin supérieur). Le territoire sélectionné pour la recherche englobait toute l'Alsace, le nord-ouest de la Suisse avec cinq cantons, ainsi qu'une grande partie du Pays de Bade et le sud de la Rhénanie-Palatinat. En raison de 
sa structure topographique et de sa situation climatique, le Rhin supérieur offre des conditions favorables à la production de biomasse.

Le bois, principale source de bioénergie, contribue à hauteur d'environ $500 \mathrm{kWh} / \mathrm{habitant} /$ an à la demande totale d'énergie spécifique à la région, estimée à $30000 \mathrm{kWh} / \mathrm{habitant/an}$. Les résidus agricoles et le lisier possèdent un potentiel théorique d'environ $200 \mathrm{kWh} / \mathrm{habitant} / \mathrm{an}$, les déchets organiques et verts de $90 \mathrm{kWh} / \mathrm{habitant} / \mathrm{an}$. Les boues d'épuration pourraient apporter environ $50 \mathrm{kWh} / \mathrm{habitant} / \mathrm{an}$. Ainsi, on pourrait théoriquement couvrir $3 \%$ des besoins totaux en énergie du Rhin supérieur en exploitant toutes les sources de biomasse.

Alors que la biomasse forestière est déjà presque intégralement exploitée et présente un potentiel supplémentaire de $10 \%$ maximum, les résidus de l'agriculture (comme la paille) et le lisier produit par l'élevage sont actuellement très peu utilisés.

Les principales formes d'exploitation énergétique de la biomasse sont la combustion du bois, la méthanisation et l'incinération des déchets. Dans la région, ces technologies sont largement utilisées depuis longtemps et ont permis le développement d'une forte compétence technique.

L'Institut de technologie de Karlsruhe (Karlsruher Institut für Technologie, KIT) a mené plus de 100 entretiens avec des experts des trois pays, en collaboration avec ses partenaires de la Fachhochschule Nordwestschweiz et de l'École nationale du Génie de l'Eau et de l'Environnement de Strasbourg. Ces entretiens ont permis de constater que dans les trois pays, le principal moteur de l'exploitation de la bioénergie réside dans les incitations financières publiques. De plus, les projets bioénergétiques, pour réussir, doivent être adaptés à la situation locale et s'intégrer dans les chaînes locales de création de valeur ajoutée et dans les réseaux d'acteurs locaux.

Lors de sondages comparatifs sur l'acceptation des installations de méthanisation entre les trois pays, on a constaté que la bioénergie arrivait partout en troisième ou quatrième place derrière l'énergie solaire, l'éolien et l'énergie hydraulique en réponse à la question sur les formes d'énergie renouvelable souhaitées pour l'avenir ${ }^{10}$.

On peut citer l'exemple de la scierie SIAT-BRAUN, à Urmatt (Alsace). Avec environ $600000 \mathrm{~m}^{3}$ de résineux traités par an, c'est la plus grosse scierie française. L'entreprise familiale créée en 1818 emploie tout juste 300 personnes. Pour garantir une rentabilité maximale et durable, un projet visant à obtenir une autonomie totale en combustible et en matière première a été mis sur pied.

Dans une centrale récemment bâtie, la biomasse (les « déchets ») permet de produire 4,8 MW d'électricité. Pratiquement toute la chaleur résiduelle est utilisée, d'une part pour le séchage des copeaux, d'autre part pour le séchage des

\footnotetext{
${ }^{10}$ Ursula Göttert, BIOPRO Baden-Württemberg GmbH, 3 août 2015.
} 
produits sciés. Avec les copeaux, la société fabrique environ 80000 tonnes de granulés par an.

\section{Une nouvelle idée : l'utilisation de la chaleur résiduelle}

Lors du deuxième congrès trinational sur le climat et l'énergie organisé par TRION pour le compte de la Conférence du Rhin supérieur dans les locaux du Landratsamt de Lörrach (Allemagne), des décideurs politiques, des personnels administratifs et des entreprises énergétiques se sont consacrés au thème spécifique des réseaux de chaleur et de l'utilisation de la chaleur résiduelle dans le Rhin supérieur.

L'utilisation de la chaleur dégagée par l'industrie et les ménages va principalement contribuer à réduire la consommation d'énergie et constitue donc un facteur important pour remplir les objectifs d'économies d'énergie du Rhin supérieur et pour réaliser la stratégie énergétique et climatique commune de la Conférence du Rhin supérieur. Le thème des réseaux de chaleur est particulièrement pertinent dans le contexte transfrontalier lorsqu'il permet d'encourager la création de réseaux de chaleur transfrontaliers.

\section{Ouverture de la loi allemande sur les énergies renouvelables (Erneuerbare-Energien-Gesetz, EEG) à l'électricité produite dans d'autres États membres de l'UE ${ }^{11}$}

Le ministère fédéral de l'Économie et de l'Énergie a présenté ses arguments pour une "ouverture partielle de l'EEG à l'électricité provenant d'autres États membres de l'UE dans le cadre de l'appel d'offres pilote pour des installations photovoltaïques au sol», et a convenu avec la Commission européenne, dans le cadre de l'autorisation de l'EEG 2014 par rapport à la législation sur les aides d'État, d'admettre à partir de 2017 la participation d'installations établies dans d'autres États membres à hauteur de $5 \%$ des capacités à installer chaque année.

Ce concept est un signal important pour les voisins européens de l'Allemagne, qui montre qu'il faut renforcer la coopération en matière de soutien aux énergies renouvelables, pour le bénéfice des deux parties. Ce concept d'ouverture reprend l'orientation européenne systématique de la transition énergétique déjà inscrite dans la loi sur le marché de l'électricité.

Cette ouverture doit également contribuer à l'ancrage transfrontalier de la transition énergétique et à une harmonisation progressive des systèmes de subvention pour les énergies renouvelables. Deux appels d'offres pilotes ouverts ont été menés avec deux pays partenaires, le Danemark et le Luxembourg.

Les subventions à l'électricité renouvelable venant de l'étranger sont soumises à trois conditions :

\footnotetext{
${ }^{11}$ Ministère fédéral de l'Économie et de l'Énergie, 12 octobre 2016.
} 
- la signature d'un accord de droit international entre l'Allemagne et le pays partenaire ;

- une coopération basée sur le principe de la réciprocité. Cela signifie que si l'Allemagne ouvre ses appels d'offres aux installations étrangères, alors à l'inverse, l'État partenaire ouvre ses appels d'offres dans la même mesure aux installations allemandes ;

- l'électricité doit avoir un effet réel sur le marché allemand de l'électricité («importation physique »).

L'expérience tirée de la mise en œuvre du concept pilote avec des installations photovoltaïques au sol doit permettre l'ouverture partielle à d'autres technologies à partir de 2017.

Dans ce domaine, la France et l'Allemagne pourraient mener ensemble un projet pilote de subvention du photovoltaïque en France, et vérifier dans quelle mesure un tel projet pilote pourrait être réalisé dans le contexte de l'arrêt programmé de la centrale nucléaire de Fessenheim dans le Haut-Rhin.

\section{Économies d'énergie - développement de la mobilité et efficacité énergétique}

La région du Rhin supérieur constitue un trait d'union important entre différents itinéraires remarquables de circulation ferroviaire et de navigation fluviale. Mais les projets transfrontaliers de transport local ont surtout contribué à améliorer l'efficacité énergétique de la mobilité et l'accessibilité pour les citoyens. L'objectif prioritaire est de rendre les offres tarifaires existantes attractives et accessibles dans tout le Rhin supérieur, et de les élargir dans le cadre de groupements transfrontaliers. À ce titre, le programme INTERREG représente une source de financement non négligeable.

Parallèlement, un soutien est apporté aux modes de transport durables, ainsi qu'aux recherches et expériences en matière de technologies innovantes dans des agglomérations frontalières (comme la mise à disposition de voitures électriques pour le personnel frontalier de la société ABB entre la Suisse et l'Allemagne).

Ces dernières années dans le Rhin supérieur, beaucoup de progrès ont été accomplis dans le domaine ferroviaire. Par exemple, une desserte régionale a été établie entre Fribourg et Mulhouse (2011). Un TGV part tous les jours de Fribourg vers Paris (2012). Un nouveau pont ferroviaire sur le Rhin entre Strasbourg et Kehl (2011) a permis d'accélérer les trajets Munich - Stuttgart - Strasbourg - Paris et Francfort - Strasbourg - Paris ou Marseille en TGV.

Avec l'Europass, les frontaliers de l'eurodistrict Strasbourg-Ortenau peuvent utiliser tous les transports publics à un tarif avantageux. Des lignes de tramway transfrontalières sont en construction entre Strasbourg et Kehl (mise en service en 2017) ainsi qu'entre Bâle et Saint-Louis (mise en service en 2019).

Un tram circule déjà entre Bâle et Weil am Rhein (2015). À partir de décembre 2016, il existera des liaisons TER directes à petit prix entre Strasbourg 
et Neustadt (Rhénanie-Palatinat) le week-end. Des trams-trains circulent à Mulhouse et dans l'agglomération de Karlsruhe. L'interconnexion entre réseau de tram et voie ferrée permet de créer des liaisons directes sans rupture de charge entre transports urbains (tramway ou métro) et réseau ferré régional. L'objectif est d'assurer un lien entre le centre-ville et la périphérie, en utilisant du matériel compatible avec différents systèmes.

Il existe enfin de nombreuses pistes cyclables qui franchissent le Rhin. Un nouvel itinéraire est en construction entre les communes de Rheinau (Allemagne) et Gambsheim (France), pour un coût de 5 millions d'euros. Un autre a été mis en service entre Bâle, Saint-Louis et Weil am Rhein.

\section{Bicyclettes à assistance électrique $(25 \mathrm{~km} / \mathrm{h})$ et « speed bikes » $(45 \mathrm{~km} / \mathrm{h})$}

Le nombre de bicyclettes électriques a désormais franchi le cap des 2,5 millions sur les routes allemandes. Le vélo électrique est un moyen de locomotion agréable (et qui crée des emplois) : il offre une solution adaptée et simple pour franchir les côtes, parcourir de grandes distances ou rouler contre le vent. Pour aller au travail, faire des courses ou se déplacer avec une remorque pour les enfants, l'assistance électrique est un atout indéniable. Pour les personnes âgées ou ayant des handicaps physiques aussi, un vélo électrique ouvre de nouvelles perspectives de mobilité. Avec l'augmentation générale du trafic cycliste et le manque de places de stationnement en ville, les deux-roues utilitaires constituent une raison supplémentaire pour en faire encore plus, à long terme, afin de promouvoir le cyclisme sur tout le territoire du Rhin supérieur, et pour resserrer encore le réseau transfrontalier de pistes cyclables.

À Golbey (région Alsace-Champagne-Ardenne-Lorraine), la société Moustache Bikes est spécialisée dans la production de bicyclettes à assistance électrique. Elle est encore jeune, mais prospère, et emploie déjà 32 personnes.

\section{L'efficacité énergétique crée des emplois}

Une étude de TRION-climate met en lumière le potentiel des rénovations de bâtiments. La rénovation rapide du parc immobilier pourrait apporter une contribution décisive à la transition énergétique et recèle un potentiel économique important (maintien des emplois existants et création de nouveaux emplois) pour les entreprises de la région.

TRION chiffre à 3,6 milliards d'euros par an les investissements à réaliser rien que pour la rénovation des enveloppes de bâtiments et des systèmes de chauffage sur le territoire du Rhin supérieur. Près des deux tiers de cette somme concernent la partie allemande, un bon quart la partie française et à peine un douzième le secteur suisse du Rhin supérieur. Plus de la moitié du marché réside dans la rénovation des enveloppes de bâtiments, et un peu moins dans le remplacement des installations de chauffage et dans les énergies renouvelables. 
Il existe des différences d'une région à l'autre : ainsi, en termes de valeur économique, le secteur des fenêtres est très important en raison du fort taux de renouvellement et du coût relativement élevé au mètre carré, principalement en Suisse. En France et en Allemagne, les toitures importent également beaucoup, ce qui est essentiellement dû à la forte proportion de maisons individuelles ${ }^{12}$.

Le président de la commission Climat et Énergie, Alberto Isenburg, juge l'évolution positive. "Aujourd'hui, la protection du climat présente le même degré de priorité en Alsace, dans le Pays de Bade et dans le nord-ouest de la Suisse. Auparavant, les Français étaient un peu à la traîne, en particulier en matière de rénovation des bâtiments pour augmenter leur efficacité énergétique. Mais la France s'est bien rattrapée ces dernières années. Aujourd'hui, il se passe beaucoup de choses dans les trois pays. Les mesures prises vont essentiellement dans la même direction, même si chacun adopte son propre rythme. Les approches varient parfois. Certains travaillent par incitations financières par exemple pour la rénovation des bâtiments -, d'autres utilisent la législation, d'autres encore misent sur le volontariat. Ce qui nous aide, c'est que nous disposons dans le Rhin supérieur d'excellents instituts de recherche qui sont toujours plus connectés ${ }^{13}$.»

\section{DE TOUTE ÉVIDENCE, QUATRE CRITÈRES sont décisifs pour progresser ${ }^{14}$ :}

- des citoyens intéressés et engagés (locataires/propriétaires de bâtiments), volontaires pour économiser l'énergie et utiliser les énergies renouvelables sont la clé du succès ;

- le commerce et l'artisanat doivent disposer d'un personnel suffisamment formé et sensibilisé pour convaincre les locataires/propriétaires des avantages des économies d'énergies et des énergies renouvelables ;

- des programmes d'aide nationaux et régionaux attirent l'attention sur les possibilités existantes, donnent de la confiance et permettent de surmonter les obstacles initiaux à l'investissement ;

- les informations sur les technologies existantes et nouvelles ainsi que sur les programmes d'aide doivent être communiquées de manière compréhensible. Elles motivent les locataires/propriétaires et assurent la transparence du marché.

Traduction de Marie-Céline GEORG

\footnotetext{
${ }^{12}$ Agence allemande de l'Énergie (Deutsche Energie-Agentur), Zukunft Haus Info, édition de mai 2013.

${ }^{13}$ Entretien avec la Mittelbadische Presse, 27 novembre 2015.

${ }^{14}$ Agence allemande de l'Énergie (Deutsche Energie-Agentur), Zukunft Haus Info, édition de mai 2013.
} 


\section{Les défis de l'Office franco-allemand pour la transition énergétique ${ }^{1}$}

\section{Sven RÖSNER}

Dans cette contribution, je présenterai dans un premier temps le rôle de l'Office francoallemand pour la transition énergétique (OFATE) dans les échanges entre la France et l'Allemagne, puis j'aborderai diverses problématiques franco-allemandes ayant trait à l'énergie.

\section{L'Office franco-allemand pour la transition énergétique}

\section{Une plate-forme d'échange franco-allemande}

L'Office franco-allemand pour la transition énergétique a été créé en 2006 à l'initiative du gouvernement français et du gouvernement allemand. L'objectif était alors d'instaurer une plate-forme d'échange public/privé franco-allemande dans le domaine de l'éolien, où les acteurs industriels et politiques pouvaient se rencontrer et parler de leur expérience. L'OFATE est situé à la fois dans les locaux du ministère de l'Environnement, de l'Énergie et de la Mer à Paris et dans ceux du ministère de l'Économie et de l'Énergie à Berlin. Son financement est porté à $50 \%$ par les gouvernements français et allemand et à $50 \%$ par ses 220 entreprises adhérentes, qui représentent l'intégralité de la chaîne de valeur de la transition énergétique.

\section{Cinq périmètres d'activités}

Jusqu'à l'été 2016, l'OFATE comprenait quatre périmètres d'activités (Énergie éolienne, Énergie solaire, Bioénergies, Systèmes \& Marchés). Or l'organisme et les deux ministères ont constaté qu'il fallait aller plus loin pour ne pas perdre en pertinence, car la transition énergétique ne consiste pas uniquement à remplacer une technologie de génération d'énergie par une autre. Il s'agit plutôt d'une transformation complète du domaine de l'énergie. Par conséquent, dans le cadre des dix ans de l'OFATE, le Conseil des ministres franco-allemand (CMFA) a annoncé en avril 2016 son élargissement à un nouveau périmètre, nommé Efficacité et Flexibilité. L'organisme va désormais se pencher sur la flexibilité du système électrique et énergétique (parce qu'il existe de nombreuses passerelles entre la chaleur et l'électricité par exemple), ainsi que sur l'efficacité énergétique dans l'industrie et chez le particulier. En effet, l'efficacité énergétique va

\footnotetext{
${ }^{1}$ Texte issu d'une communication donnée dans le cadre du colloque Coopérations franco-allemandes dans la perspective d'une politique européenne de l'énergie, corganisé par le CIRAC les 26 et 27 septembre 2016, $\mathrm{NdE}$.
} 
avoir un impact sur la production de l'énergie et la façon dont elle doit être acheminée vers les foyers. Il s'agit en outre de s'intéresser aux possibilités de transport d'électricité.

\section{Conférences et publications}

L'OFATE organise une vingtaine de conférences par an, offrant la possibilité aux acteurs issus de l'industrie, des administrations (ministères et organismes de régulation), de la finance, de la recherche et de la société civile de nouer des contacts pour travailler ensemble sur de nouvelles solutions innovantes. Depuis sa création, l'OFATE a permis l'établissement de 5000 contacts entre acteurs. Dans le domaine de la recherche par exemple, Mines ParisTech et l'Institut Fraunhofer pour l'énergie éolienne et les technologies des systèmes énergétiques (Fraunhofer-Institut für Windenergie und Energiesystemtechnik, IWES) ont établi un système pour simuler les interactions entre les systèmes énergétiques français et allemand, en tenant compte de l'intermittence des énergies éolienne et photovoltaïque. Cette analyse a permis de définir la robustesse du système actuel et de voir où il fallait investir dans les années à venir pour rendre ce système plus flexible.

L'OFATE répond chaque année à environ 400 demandes des ministères et des entreprises adhérentes sur des sujets techniques, juridiques et pratiques. Il ne s'agit pas d'une agence de communication sur la transition énergétique, mais d'un organisme qui essaie de montrer les possibilités de la transition énergétique tout en évoquant les problèmes qui ont été rencontrés.

L'organisme compte de nombreuses PME parmi ses adhérents, auxquelles il propose une veille régulière au niveau réglementaire, économique et technique. Ces quelque 65 documents publiés chaque année s'adressent également aux administrations.

\section{Un rôle d'intermédiaire et de consultant}

L'OFATE joue un rôle d'intermédiaire pour les gouvernements, en participant aux échanges entre la direction générale de l'Énergie et du Climat en France et le ministère allemand de l'Économie et de l'Énergie sur le cadre réglementaire et son évolution dans le domaine de l'énergie dans les deux pays. Il organise des soirées politiques où les responsables institutionnels peuvent échanger avec la société civile.

Enfin, il agit en tant que consultant dans le cadre de la plate-forme énergétique franco-allemande de l'Agence française de l'environnement et de la mâ̂trise de l'énergie (ADEME) et de l'Agence allemande de l'énergie (Deutsche Energie-Agentur, dena), créée en 2015 à l'initiative du Conseil des ministres franco-allemand. L'objectif de cette plate-forme est de faciliter les coopérations entre entreprises françaises et allemandes à travers différentes initiatives, comme par exemple la création d'un prix attribué à un projet particulièrement 
intéressant. Ce prix a été remis pour la première fois en juin 2016. D'autres projets qui sont en préparation, comme des séminaires qui peuvent permettre d'apprendre comment travailler avec des acteurs issus du pays voisin.

\section{Défis franco-allemands en matière d'énergie}

\section{Intermittence du photovoltaïque et de l'éolien}

L'OFATE a organisé différentes conférences sur la question de la flexibilité. Les énergies intermittentes comme l'éolien et le solaire ne produisent pas nécessairement de l'électricité au moment où le consommateur en a le plus besoin. Il est possible de réduire le besoin de flexibilité en créant un mix relativement équilibré entre le solaire, qui produit le plus d'électricité à midi, et l'éolien, dont la production est répartie sur toute la journée, ou encore en utilisant le biogaz, réglable comme une centrale à gaz, ou la géothermie. Sur le plan du stockage, il existe en outre les stations de transfert d'énergie par pompage (STEP) ou le stockage en batteries.

Néanmoins, selon l'entreprise RTE (Réseau de transport d'électricité), l'évolution des énergies renouvelables telle que prévue par la programmation pluriannuelle de l'énergie (PPE) fait qu'il ne sera pas nécessaire de recourir à des moyens de stockage avant 2030. Pour RTE, le moyen de flexibilité le moins cher réside dans le développement du réseau de transport d'électricité et la multiplication des interconnexions en Europe.

\section{Captage et stockage du $\mathrm{CO}_{2}$ (carbon capture and storage, CCS)}

Le CCS rencontre non seulement un problème d'acceptabilité par la population, mais aussi et surtout un problème de rentabilité dans le secteur thermique pour les opérateurs historiques comme RWE, E.ON et Vattenfall. Cette dernière entreprise cherche actuellement un repreneur pour ses mines de lignite pour un euro symbolique. Si l'on ajoute le coût d'une technologie telle que le CCS, le calcul devient encore moins intéressant. Dès lors, il ne sera pas nécessaire de stocker le $\mathrm{CO}_{2}$ car celui-ci ne sera pas émis en raison du fait que la centrale ne sera plus rentable et qu'elle sera remplacée par autre chose. C'est la tendance aujourd'hui sur le marché, avec un prix de l'électricité qui oscille autour de 3 centimes d'euros par kWh.

\section{Efficacité énergétique}

En matière d'efficacité énergétique, le législateur allemand s'intéresse beaucoup au cadre contraignant qui a été mis en place en France. L'OFATE reçoit régulièrement des questions des services du ministère fédéral de l'Économie et de l'Énergie à ce sujet. La transition énergétique en Allemagne demeure très axée sur le développement des énergies renouvelables, puis sur le transport et la distribution de l'électricité. En revanche, le pays n'est pas en avance en ce qui con- 
cerne l'efficacité énergétique. Par conséquent, les responsables allemands trouvent très intéressant le système français, et notamment le fait que les municipalités puissent définir des objectifs dans lesquels elles incluent non seulement la production, mais aussi la consommation d'énergie. On arrive à l'idée que c'est bien de développer les énergies renouvelables, mais que c'est encore mieux de ne pas en avoir besoin. La solution la moins chère est en effet de permettre à la population de faire des économies d'énergie.

Selon moi, il faut trouver des moyens pour valoriser les investissements réalisés dans le cadre de l'isolation thermique des foyers, notamment en cas de revente d'un bien immobilier dans les années qui suivent les travaux. C'est un des grands défis pour le succès de la transition énergétique en France et en Allemagne. En revanche, cela fonctionne déjà dans le tertiaire. La société Unilever, par exemple, a signé un bail pour de nouveaux locaux en Île-de-France, qui ne porte pas seulement sur les murs, mais aussi sur la consommation de l'énergie pour les 20 prochaines années. Aujourd'hui, de plus en plus de baux portent également sur la consommation.

En France, la loi sur la transition énergétique comprend tout de même un programme ambitieux de rénovation de 500000 appartements. Cela peut fournir la masse critique pour démarrer un processus, engendrant ainsi une prise de conscience et une baisse des coûts qui permettrait par la suite de rendre ces solutions plus intéressantes pour le grand public.

\section{Sociétés franco-allemandes dans l'énergie}

Dans le cadre de la transition énergétique, des sociétés se sont forgé des compétences interculturelles leur permettant de travailler dans les deux pays, particulièrement dans les services. On voit cela par exemple dans le domaine de l'agrégation. Il s'agit d'entreprises qui commercialisent à la bourse les énergies renouvelables pour les propriétaires des centrales.

\section{Rôle du citoyen}

Je suis convaincu que la transition énergétique est un projet sociétal qui traite entre autres de l'énergie et qui redistribue les cartes au niveau de l'industrie, de la recherche, de la formation et de l'emploi. Il est essentiel de ne pas oublier le rôle du citoyen, dont dépend la réussite de ce projet. En Allemagne, une enquête publiée en septembre 2016 montre que $92 \%^{2}$ des sondés sont favorables à la transition énergétique. En revanche, une autre enquête révèle que l'acceptabilité est moindre pour ce qui est du développement du réseau de transport d'électricité. Il nous faut travailler sur ce point dans les deux pays.

\footnotetext{
${ }^{2}$ PwC, Energiewende 2015 [en ligne], Francfort-sur-le-Main, mars 2015. Disponible sur : https://www.pwc. de/de/energiewende/assets/onlinestudie-energiewende.pdf [consulté le 27/04/2017].

${ }^{3}$ Erneuerbare Energien Hamburg Clusteragentur $\mathrm{GmbH}$, Norddeutsche sind die stärksten Befürworter der Energiewende [en ligne], Hambourg, 15 février 2016. Disponible sur: http://www.erneuerbare-energien-
} 


\section{Réseau de transport d'électricité}

Le problème d'acceptabilité du développement du réseau par la population se pose dans de nombreux pays. Dès lors, un projet de développement de réseau prend sept ans : six ans de concertation et un an de construction. En Allemagne, le plan de développement du réseau (Netzausbauplan, NAP) rassemble 36 projets, dont actuellement trois grandes lignes à haute tension. En effet, l'électricité produite à partir des éoliennes installées au bord de la mer du Nord et de la mer Baltique doit être transportée vers le sud du pays, fortement industrialisé. Il y a un gros travail d'explication à mener avec les riverains. L'accord trouvé consiste à enterrer ces lignes, ce qui a un impact non négligeable sur les coûts. La livraison n'est plus prévue pour 2022, date à laquelle vont être mises hors service les dernières centrales nucléaires en Allemagne, mais pour 2025. Si durant cette période de transition, l'Allemagne pourra compter sur les interconnexions avec les pays voisins, il s'agit tout de même d'un stress test pour le réseau de transport.

\section{Propriété intellectuelle}

Il est nettement plus facile de mettre en place aujourd'hui un projet francoallemand dans le domaine de la recherche que dans le domaine de l'industrie, fortement concurrentiel. De plus, certaines technologies sont devenues standardisées : il est par exemple beaucoup moins cher de produire des panneaux photovoltaïques en Chine. Enfin, en ce qui concerne les batteries, ce n'est pas seulement une question de prix, mais de compétences. Les acteurs en Corée du Sud sont beaucoup plus en avance sur le stockage lithium-ion. Il est donc plus intéressant en Europe d'acheter ces batteries et de construire autour un système de gestion qui représente une valeur ajoutée.

LA COOPÉRATION FRANCO-ALLEMANDE EST SOLIDE et joue actuellement un rôle qui n'a jamais été aussi important en Europe. Je crois en son avenir pour le bien de la France, de l'Allemagne et de l'Europe. Pour ce qui est de la transition énergétique, il serait utile de faciliter à l'avenir les échanges entre l'Allemagne et la France dans le domaine de la recherche. Il me semble très important de se parler et d'apprendre de l'autre au niveau local et au-delà, avec peut-être un impact direct sur les économies de nos pays.

hamburg.de/de/service/pressemitteilungen/details/norddeutsche-sind-die-staerksten-befuerworter-der-energie wende.html [consulté le 27/04/2017]. 

- IV -

\section{DÉFIS ET TRANSFORMATIONS}

DU MARCHÉ DE L'ÉLECTRICITÉ 



\section{Compétitivité : la transition énergétique menace-t-elle nos sites de production ?'}

\section{Joachim LANG}

Depuis plus d'un siècle, notre branche gagne sa vie en fournissant de l'énergie à la population. L'essentiel a toujours été de produire, transporter et fournir de l'électricité et du gaz avec efficacité et fiabilité. C'était le cœur commercial de notre activité, et ça l'est encore aujourd'hui. Jusqu'à présent, les données que nous collections dans ce cadre n'étaient qu'accessoires: elles servaient essentiellement à traiter les opérations commerciales. Grâce aux solutions économiques de stockage et aux nouvelles possibilités d'analyse, les données ont acquis une valeur commerciale propre. En effet, elles permettent d'offrir de nouveaux services aux clients. Les données sont devenues précieuses, bientôt plus précieuses peut-être que l'énergie dont la livraison a généré leur existence. On peut tout à fait envisager de fournir de l'électricité ou du gaz comme on propose aujourd'hui de nombreuses applications de smartphone : apparemment gratuites, mais en réalité payées par les données du client. En bref: du gaz ou de l'électricité gratuitement, à condition que le client renonce à sa vie privée. Utopique ? Peut-être. Impensable ? Certainement pas.

\section{Évolution de l'environnement concurrentiel et disruption}

Ces nouveaux enjeux changent radicalement l'environnement concurrentiel de notre branche. Si les performances numériques sont devenues un facteur de compétitivité décisif, le client ne va plus comparer les fournisseurs d'électricité entre eux. Au lieu de cela, il va nous comparer avec des banques et des assurances, avec Google et Amazon.

Simultanément, des prestataires extérieurs à la branche peuvent pénétrer dans le domaine énergétique en proposant des prestations numériques attractives. Pour l'économie de l'énergie, cela signifie qu'il ne suffit plus d'être bon dans son activité énergétique proprement dite. Il est au moins aussi important de maîtriser la génération des données, leur analyse, et la numérisation des processus, afin de s'en servir pour concevoir des offres attractives pour les clients. Sinon, d'autres le feront.

Voilà ce qu'est l'avenir, et c'est déjà le présent : l'énergie va devenir un accessoire, tout comme le lait accompagne les céréales au petit-déjeuner. Elle doit être disponible, de bonne qualité et aussi bon marché que possible - ce qu'elle était déjà. Le produit que le client connaît et apprécie, ce sont les céréales.

Est-ce un diagnostic trop sombre de l'économie énergétique ? On devrait être de cet avis si on suit le concept de la disruption tel qu'il a été développé il y a 20 ans. Selon ce point de vue, les entreprises établies ont très peu de chances de se maintenir sur un marché ciblé par une innovation disruptive.

\footnotetext{
${ }^{1}$ Article rédigé en mars 2016, NdE.
} 
En effet, elles devraient pour cela remettre en question précisément ce qui leur a permis d'être grandes, fortes et performantes. Elles devraient être prêtes à cannibaliser leur propre activité. Elles devraient être en mesure de se réinventer.

Tout cela n'est pas facile. C'est d'autant plus difficile lorsqu'on maîtrisait particulièrement bien son activité précédente, parce qu'on ne peut pas s'empêcher de penser avant tout à ce qui a fonctionné pendant des décennies. Comme on dit familièrement, « on prend les mêmes et on recommence ». Pourquoi cela devrait-il subitement cesser de fonctionner?

C'est précisément la tentation fatale à laquelle les entreprises établies ont beaucoup de mal à résister. La guerre entre les entreprises énergétiques établies et les nouveaux arrivants numériques est-elle déjà terminée avant même d'avoir commencé ?

Absolument pas! Les prophéties apocalyptiques ne se justifient pas. Le fatalisme n'est pas une nécessité. Il faut plutôt le courage de rompre avec le type traditionnel du fournisseur d'énergie intégré : celui-ci a fait son temps. Comment, en tant qu'exploitant de grandes centrales et marchand de grandes quantités d'énergie, peut-on aider ses clients de manière crédible à trouver des solutions décentralisées, proches d'eux, et à utiliser plus efficacement l'énergie?

\section{Une spécialisation dans les énergies nouvelles et les solutions client}

Chez E.ON, nous divisons très clairement nos activités entre les énergies classiques et les énergies nouvelles. La direction dans laquelle nous avons choisi d'orienter notre nouvelle stratégie est visible dans notre slogan : Empowering customers, shaping markets (donner du pouvoir aux clients, modeler les marchés). Nous avons regroupé dans une entreprise spécifique, sous le nom d'Uniper, les activités relatives aux énergies classiques, en particulier les centrales conventionnelles et le marché global de l'énergie. E.ON, de son côté, se concentrera totalement sur les énergies renouvelables, les réseaux énergétiques et les solutions client.

Ce nouvel avenir énergétique a déjà commencé et prend de la vitesse. Centrales solaires et stockages toujours plus performants, numérisation de tout l'approvisionnement énergétique et nouvelles prestations associées, électromobilité, Power-to-Heat, smart grids avec la mise en réseau intelligente des infrastructures énergétiques: tout cela va révolutionner l'approvisionnement énergétique. Ce n'est plus la vente de kilowatts qui prime, mais la fourniture de solutions énergétiques globales adaptées aux besoins de nos clients.

À l'avenir, E.ON va résolument axer son travail sur ses clients, pour optimiser et numériser tous les processus en fonction de leurs exigences. Le moteur de cette transformation sera notre nouvelle organisation marketing centrale (CMO). Nous y intégrerons et y coordonnerons toutes les fonctions marketing pour l'ensemble des activités du groupe, par exemple le développement de pro- 
duits innovants, la communication avec les clients, l'analyse du marché et la gestion de la marque. Tous les processus associés seront harmonisés à l'échelle du groupe. Cette nouvelle unité réunira également les capacités numériques du groupe et promouvra la transformation numérique.

Pour cela, nous mettons notamment en place un Future Lab qui élaborera des projets disruptifs reposant sur l'exploitation de données. Nous allons radicalement raccourcir le chemin qui mène d'une bonne idée à un produit convaincant. Pour cela, il faut prendre des décisions rapides et agiles, définir des responsabilités claires et disposer d'une culture d'entrepreneuriat. En fin de compte, il s'agit de sortir d'une posture défensive face aux nouveaux défis, pour passer à l'action. Ceux qui veulent seulement défendre leur activité actuelle ont déjà perdu. À l'inverse, ceux qui considèrent les nouvelles possibilités comme des chances d'entreprendre peuvent les convertir en croissance et en création de valeur ajoutée.

\section{Prise de participation dans des start-up}

Mais pour cela, il faut être prêt à apprendre, par exemple auprès de start-up. Les cultures et les processus des entreprises établies et des start-up ne sont pas des opposés inconciliables. Chacun peut apporter ses atouts à un projet commun : la force d'innovation d'un côté, la masse critique de l'autre. Nous faisons partie des premiers à nous être implantés dans la Silicon Valley avec notre propre personnel. Par des co-investissements stratégiques, nous accédons volontairement non seulement à de nouvelles idées technologiques, mais aussi à de nouvelles impulsions culturelles.

Nous prenons des participations dans des start-up pour apprendre, pas pour les dominer. C'est pour cela que nous laissons toute latitude à nos partenaires, et que nous les aidons à développer leurs idées. Nous partageons les risques et les chances : si nous réussissons ensemble, la valeur de la start-up augmentera et nous pourrons proposer de nouvelles solutions à nos clients.

En 2013, Thermondo a été le premier chauffagiste en ligne d'Allemagne. Aujourd'hui, il est le plus gros acteur du marché. Ce fournisseur allie la rapidité et l'étendue de l'offre d'une entreprise sur Internet à l'exceptionnel savoir-faire d'un chauffagiste expérimenté. Grâce à une plate-forme en ligne et à une infrastructure informatique propre à l'entreprise, les clients peuvent comparer différents fabricants de systèmes de chauffage et diverses technologies, rapidement, facilement et à moindres frais.

Un autre exemple est greenwave. Dans le nouveau monde énergétique des solutions intelligentes pour les particuliers et les entreprises, les locaux des clients contiendront une multitude d'appareils interconnectés. De plus en plus de données vont être échangées pour optimiser l'utilisation, la combinaison et le pilotage des appareils. Ceux-ci doivent donc parler la même langue informatique, ce qui est loin d'être le cas à l'heure actuelle. C'est pourquoi des sys- 
tèmes spécifiques, passerelles ou switchs, assurent la communication. À l'avenir, le client pourra consulter les données collectées sur des portails en ligne ou des smartphones. Ainsi, il y aura accès en permanence et pourra piloter ses appareils comme il le souhaite. Nous envisageons l'intégration de tous ces équipements informatiques dans les habitations de nos clients. Avec des partenaires comme greenwave, nous voulons saisir l'occasion de simplifier la connexion et le pilotage d'un grand nombre d'applications, et de proposer de nouvelles prestations à nos clients. Par exemple, il peut s'agir de commander le chauffage ou de connaitre en toute transparence la consommation propre de l'électricité produite par les panneaux photovoltaïques de nos clients.

Début novembre 2015, nous avons également pris une participation dans la start-up Bidgely. Cette jeune pousse américaine propose aux entreprises des applications énergétiques dans le cloud, consultables sur Internet ou par téléphone mobile en temps réel. Ainsi, les ménages peuvent mieux gérer leur consommation d'énergie. De plus, ils reçoivent par exemple des conseils pour optimiser leurs appareils électriques et de chauffage, envoyés directement sur leur téléphone mobile. Ils peuvent aussi comparer leur consommation d'énergie à celle de ménages comparables dans leur voisinage.

\section{Numérisation et valeur ajoutée}

Comme le montrent ces exemples, nous abordons la numérisation de bien des manières. Certaines choses fonctionnent déjà très bien, d'autres tâtent encore le marché pour comprendre ce que les clients veulent et ce qu'ils ne veulent pas ou pas encore. En fin de compte, ce qui réussit n'est pas ce que nous préférons, mais ce qui apporte une valeur ajoutée au client. D'un autre côté, les souhaits des clients ne tombent pas du ciel : ils naissent au moment où les clients voient ce qui est possible. C'est cela que nous voulons montrer, et développer avec et pour les clients.

Les entreprises établies peuvent répondre efficacement aux tendances disruptives générées par la numérisation. John Hagel, qui connaît la question en profondeur, a écrit: "En ces temps de mutations exponentielles, celui qui se laisse séduire par les sirènes de la Silicon Valley ou qui contemple avec trop de fascination l'échec d'autres entreprises établies risque d'être détourné des nouvelles occasions et de les laisser passer ${ }^{2} . »$

En nous concentrant clairement sur l'organisation de l'avenir énergétique, nous tournons notre regard vers ces nouvelles occasions, et nous les saisissons dans le domaine de la numérisation via une multitude d'activités. Nous connais-

\footnotetext{
${ }^{2}$ John Hagel, John Seely Brown, Maggie Wooll et Andrew de Maar, Patterns of Disruption - Anticipating Disruptive Strategies in a World of Unicorns, Black Swans, and Exponentials [en ligne], Deloitte University Press, [s.1.], 2015, p. 21. Disponible sur : https://www2.deloitte.com/content/dam/Deloitte/nl/Documents/technology/ deloitte-nl-deloitte-patterns-of-disruption.pdf [consulté le 01/08/2016].
} 
sons nos points forts et nous élaborons des réponses entrepreneuriales pour nos marchés et nos clients.

LA TRANSITION ÉNERGÉTIQUE EST UN CATALYSEUR. Elle oblige, tôt ou tard, toutes les entreprises actives sur le marché de l'énergie à se confronter à un avenir totalement nouveau. Seuls ceux qui sont prêts à s'adapter en profondeur à ces modifications resteront compétitifs et pourront y prendre part. Plus vite nous trouverons des réponses à ces défis en Europe, mieux nous pourrons en profiter.

Traduction de Marie-Céline GEORG 



\section{Conséquences de la transition énergétique sur le prix pour le consommateur ${ }^{1}$}

\section{Christophe LEININGER}

Les missions de la direction du Développement des marchés de la Commission de régulation de l'énergie (CRE) concernent notamment les prix et tarifs appliqués au consommateur final (particulier et professionnel), les coûts de production sous-jacents à ces tarifs et les dispositifs de soutien au développement des énergies renouvelables (ENR). C'est notamment sur le fondement de ces trois missions que je traiterai, dans la présente contribution, des conséquences à moyen terme de la transition énergétique sur le prix pour le consommateur final.

\section{La France reste marquée par les engagements du passé}

Je souhaiterais dans un premier temps rappeler deux éléments de contexte importants en France. Le premier a trait à l'ouverture du marché de l'électricité à la concurrence initié il y a 15 ans. Nous sommes actuellement à un tournant puisque, sous la pression de la Commission européenne, le gouvernement français a annoncé en 2010, avec la loi portant nouvelle organisation du marché de l'électricité (dite loi Nome), la suppression au 31 décembre 2015 des tarifs réglementés de vente pour les clients professionnels, ce qui concerne à peu près deux tiers de la consommation d'électricité. Cette suppression va dès lors exposer ces gros consommateurs au prix du marché de gros de l'électricité. Par conséquent, les variations du prix de marché vont directement se répercuter sur les factures des consommateurs concernés. Pour les consommateurs résidentiels, les tarifs réglementés ne sont pas supprimés mais ils sont proposés par la CRE qui veillera à ce qu'ils soient suffisamment élevés pour permettre à la concurrence de se développer. Ces tarifs seront eux aussi largement inspirés du marché puisqu'ils en reflèteront pour une large part les variations. Désormais en France, la facture du consommateur sera pour partie orientée vers les évolutions du prix de marché.

Le deuxième élément est lié au fait que si la loi de transition énergétique fixe des objectifs ambitieux en matière de développement des énergies renouvelables, elle prévoit de maintenir une partie du parc nucléaire en exploitation et d'abaisser progressivement la part du nucléaire dans la production d'électricité à $50 \%$ à l'horizon 2025 (contre $75 \%$ en 2015). Cela signifie que les coûts du nucléaire auront un impact durable sur la facture des consommateurs français, voire européens, le réseau de transport d'électricité étant fortement

\footnotetext{
${ }^{1}$ Texte issu d'une communication donnée dans le cadre de la journée d'étude La transition énergétique en France et en Allemagne : défis et transformations du marché de l'électricité, coorganisée par le CIRAC le 24 septembre 2015, NdE.
} 
interconnecté à l'échelle du continent. D'autre part, les coûts du parc hydraulique installé depuis de nombreuses années en France - qui représente un atout pour la France -, vont également évoluer et continuer de se répercuter sur la facture des consommateurs.

Ainsi, les engagements du passé continueront à structurer une grande partie du mix énergétique du pays et présentent une tendance inflationniste des coûts de production.

\section{Le parc nucléaire est confronté à des enjeux de sûreté qui viendront modifier sa compétitivité}

La durée d'exploitation d'une centrale nucléaire est de 30 ans en moyenne. L'objectif d'Électricité de France (EDF), opérateur historique et fournisseur principal français, est de porter cette durée à 60 ans. Cela s'accompagne d'enjeux liés à la maintenance et à la sûreté. En termes de maintenance, il s'agit de progressivement remplacer des composants qui ont 30 ans de durée de vie par de nouveaux composants pour espérer atteindre 60 ans. En termes de sûreté, il faut l'accord de l'autorité de sûreté pour poursuivre l'exploitation d'une centrale au-delà de 40 ans, ce qui concerne actuellement une partie du parc. Dans le cas contraire, la centrale s'arrête.

Les coûts élevés liés aux enjeux de maintenance et de sûreté auront un impact sur le prix à payer par le consommateur. Avec la loi Nome, le législateur français a en effet mis en place jusqu'en 2025 l'accès régulé à l'électricité nucléaire historique (ARENH) pour les fournisseurs alternatifs. Le prix de l'ARENH, calculé et fixé par la CRE, tient compte du coût de production de l'électricité par les centrales nucléaires. Dès lors, si le tarif de l'ARENH augmente tout en restant inférieur au prix du marché de gros, les hausses de coûts auront une nette incidence sur la facture des consommateurs.

\section{Le parc hydraulique, deuxième maillon de compétitivité}

Le deuxième point susceptible d'occasionner des hausses de coûts a trait aux concessions hydrauliques. La Commission européenne demande avec insistance à la France de bien vouloir procéder à la remise en concurrence des concessions hydrauliques pour les attribuer à des fournisseurs concurrents d'EDF. Cela signifie que ces concessions, qui s'inscrivaient auparavant dans une logique tarifaire, au sens de la couverture des coûts comptables, vont aller vers une logique de vente aux prix de marché, s'accompagnant vraisemblablement d'un effet inflationniste.

\section{La poursuite du développement des énergies renouvelables}

Le troisième point concerne le développement des ENR. On observe en France une forte augmentation de la contribution au service public de l'électricité 
(CSPE). Toutefois, son niveau n'est aujourd'hui pas suffisant pour couvrir les charges liées au développement des énergies renouvelables, ce qui occasionne une dette assez importante pour EDF qui en supporte le coût.

Comme en Allemagne, des dispositifs d'exonération de la taxe s'appliquent à quelques grands consommateurs. Les plafonds appliqués sont actuellement examinés par la Commission européenne qui souhaite que des évolutions soient apportées à ce type d'exonération. Sous l'impulsion de la Commission mais aussi du gouvernement français, la CSPE est en cours de réforme. Il est probable que l'assiette de prélèvement de cette taxe soit élargie à l'essence, au diesel, au gaz et au fioul en plus de l'électricité, ces énergies participant aussi aux émissions de gaz à effet de serre et donc pouvant éventuellement être contributrices de la subvention au développement des énergies renouvelables ${ }^{2}$.

Pour CONCluRe, NOUS SOMMES FACE À UNE SITUATION de coûts inflationnistes. La question de la compétitivité doit s'apprécier à l'aune de l'augmentation des coûts, en tout cas en ce qui concerne la France. Certes aujourd'hui, le prix de marché de l'électricité est bas du fait des conditions macroéconomiques, mais cela n'est vraisemblablement que conjoncturel. Dès lors, dans un contexte de coûts inflationnistes et avec la poursuite du développement des ENR, il est susceptible de remonter. C'est dans le cadre de ce débat que s'inscrit la question de la compétitivité.

\footnotetext{
${ }^{2}$ Depuis cette communication, le projet de loi de finances rectificative pour 2015 , en date du 13 novembre 2015, a introduit la réforme de la CSPE, NdE.
} 



\section{Le rôle des réseaux dans la réussite de la transition énergétique ${ }^{1}$}

\section{Hervé MIGNON}

Cette contribution vise à montrer l'articulation entre les tendances de la transition énergétique et les évolutions nécessaires des infrastructures de réseau, tant en termes d'adaptation et de création de nouveaux ouvrages que de gestion du système dans sa globalité. Je l'illustrerai par quelques exemples du côté français, qui montrent également l'interdépendance qui existe au sein du système électrique européen - le plus interconnecté au monde.

\section{Le réseau, vecteur du développement des énergies renouvelables}

En France, un événement nouveau a marqué l'agenda législatif à l'été 2015, avec l'adoption et la promulgation de la loi relative à la transition énergétique pour la croissance verte. Cette loi représente l'aboutissement de deux ans de concertations concernant les modalités de la transition énergétique et d'un an de débats parlementaires au niveau de l'Assemblée nationale et du Sénat.

La loi offre un nouveau cadre pour l'ensemble des éléments constitutifs de la transition énergétique en France. Comportant 215 articles, elle fixe de grands objectifs qui portent sur des horizons temporels et des axes différents. Il y est notamment question de réduire de $40 \%$ les émissions de gaz à effet de serre entre 1990 et 2030, de réduire de moitié la consommation d'énergie à l'horizon 2050 par rapport à 2012 et de porter la production d'énergie renouvelable à $32 \%$ de la consommation énergétique finale en 2030 , ce qui, appliqué à l'électricité, devrait correspondre à $40 \%$ en termes de consommation. Un autre objectif consiste à diversifier la production d'électricité et baisser à $50 \%$ la part du nucléaire à l'horizon 2025.

Tous ces éléments montrent que la dynamique en cours, qui porte à la fois sur les modes de production et sur les modes de consommation, va s'accélérer. Au-delà des aspects de coût va se poser la question du calendrier de mise en œuvre de ces différentes dispositions. On pourrait avoir le sentiment trompeur qu'on se situe à des horizons extrêmement lointains $(2025,2030 \ldots)$. En fait, les délais liés à la construction de réseaux de transport d'électricité, à la création de nouveaux moyens de production ou à un changement de localisation des moyens de production nécessitent un travail d'anticipation qui pousse à avoir dès à présent un regard concret sur des horizons aussi lointains.

\footnotetext{
${ }^{1}$ Texte issu d'une communication donnée dans le cadre de la journée d'étude La transition énergétique en France et en Allemagne: défis et transformations du marché de l'électricité, coorganisée par le CIRAC le 24 septembre 2015, NdE.
} 
Deuxième constat : même si ces éléments portent sur des aspects très divers, le réseau de transport d'électricité aura incontestablement un rôle important à jouer en tant que vecteur pour pouvoir accompagner et faciliter une telle transition énergétique compte tenu des différents impacts que cela peut engendrer, tant au niveau de la consommation que de la production.

En France, une tendance de fond est d'ores et déjà en cours concernant la diversification du mix énergétique, avec le développement croissant des énergies renouvelables. Cela concerne notamment l'éolien terrestre, avec plus de 9000 MW de puissance installée en 2014. Cela correspond à peu près à la situation de l'Allemagne il y a 10 ou 15 ans, avec une spécificité française qui réside dans la très forte diversification des lieux d'implantation de cette nouvelle source d'énergie. En effet, en France, il existe trois régimes de vents différents et décorrélés qui concernent la façade nord, la façade atlantique et la zone autour du Rhône. Ces trois zones de vents correspondent à trois zones de localisation prioritaires pour les nouvelles fermes éoliennes.

Au-delà de la source d'énergie, de son mode de fonctionnement et de son coût, il importe aussi d'être en capacité de mettre en connexion les moyens de production et les zones de consommation. Or la localisation différente des sources de production conduit à changer aussi structurellement le mode de développement des infrastructures de réseau. Un travail d'élaboration de nouvelles capacités d'accueil est actuellement en cours. L'éolien terrestre - mais c'est aussi vrai pour le photovoltaïque -, apparaît dans des zones qui historiquement n'étaient pas toujours desservies par le réseau de transport d'électricité.

Avec l'implantation annuelle d'environ $1 \mathrm{GW}$ de puissance éolienne terrestre, l'objectif de $15 \mathrm{GW}$ fin 2018 devrait être quasiment atteint. En revanche, en ce qui concerne le photovoltaïque, la dynamique a été plus rapide que ce qui avait été initialement prévu dans les différents travaux parlementaires antérieurs à la loi. Cette filière a été marquée successivement par des cycles d'accélération et de retournement importants, en France comme dans le reste des pays de l'Union européenne, au gré de l'évolution des dispositifs de soutien. Indépendamment des contextes de subvention, d'appels d'offres ou d'obligations d'achat quand il peut en bénéficier, ce secteur a connu une évolution très forte, le parc installé atteignant $6 \mathrm{GW}$ en 2014, et ce en l'espace de quelques années. Comme c'est le cas pour chacun des pays européens, un certain «héliotropisme » caractérise les implantations photovoltaïques en France. Le photovoltaïque se développe plutôt dans le sud de la France, où des aménagements de réseau sont nécessaires pour pouvoir gérer correctement ces nouveaux flux.

À l'heure où la COP21 va bientôt être accueillie à Paris, la question de la réduction des émissions de $\mathrm{CO}_{2}$, qui fait partie des objectifs de la loi relative à la transition énergétique, revêt un aspect particulier. Les émissions de $\mathrm{CO}_{2} \mathrm{du}$ secteur électrique en France peuvent en effet avoir des caractéristiques différentes d'autres émissions de $\mathrm{CO}_{2}$ dans d'autres pays de l'Union européenne et 
au niveau mondial. Les évolutions structurelles du parc de production conduisent à une diminution des émissions. En 2014, dernière année de référence, on a même pu assister à une diminution de plus de $40 \%$ des émissions de $\mathrm{CO}_{2} \mathrm{du}$ secteur électrique français. C'est aussi lié en partie à des températures extrêmement clémentes en hiver et frâiches en été, qui ont conduit à une moindre utilisation de l'électricité. Quoi qu'il en soit, pour la première fois en France, les énergies renouvelables (éolien, photovoltaïque et hydraulique) ont dépassé le parc thermique classique en termes de production.

Un autre point important concerne la gestion future des systèmes électriques, intégrant de plus en plus la notion de flexibilité. Au-delà de leur raccordement, le développement massif de ces nouvelles sources de production variables (par opposition aux moyens de production pilotables qui prédominaient jusqu'ici) conduit les acteurs à s'interroger sur la gestion du système électrique. La problématique de la flexibilité est l'un des grands enjeux induits par le développement du parc renouvelable. Soumises par nature aux conditions climatiques - vent, ensoleillement -, les caractéristiques des productions renouvelables modifieront de plus en plus profondément le fonctionnement du système électrique, soulevant notamment des questions de flexibilité. Les productions photovoltaïque et éolienne ont des impacts différents sur la variabilité de la consommation résiduelle : le photovoltaïque affecte principalement sa variabilité journalière, et l'éolien, sa variabilité hebdomadaire. Les estimations de l'entreprise Réseau de transport d'électricité (RTE) montrent qu'à l'horizon 2030, pour le scénario «Nouveau mix » du Bilan prévisionnel $2014^{2}$, le besoin de flexibilité hebdomadaire, essentiellement lié à la variabilité de l'éolien, s'accroîtra plus rapidement que le besoin de flexibilité journalier. La mutualisation entre pays européens via les interconnexions permettra de diminuer ces besoins de flexibilité, notamment grâce au foisonnement des productions d'énergies renouvelables.

Ceci illustre concrètement le fait que de plus en plus, le système électrique sera caractérisé par des besoins de flexibilité auxquels il conviendra de répondre via des outils concrets. Il pourra s'agir de mettre en œuvre des mécanismes de marché qui permettent d'orienter vers telle ou telle solution technologique, de gérer la demande, de gérer du stockage ou encore d'avoir de la commandabilité sur les outils de production.

Aujourd'hui, les études réalisées dans le cadre de la programmation pluriannuelle de l'énergie montrent que nous allons être davantage confrontés à des enjeux de variabilité hebdomadaire, compte tenu du mode de production éolien, qu'à des enjeux de variabilité journalière, davantage liée au photovoltaïque. Ce défi de demain doit être relevé pour pouvoir accompagner la transition énergétique de manière sûre, efficace et économique.

\footnotetext{
${ }^{2}$ RTE, Bilan prévisionnel de l'équilibre offre-demande d'électricité en France - Édition 2014, La Défense, [s.d.].
} 


\section{Les enjeux sur la sécurité d'approvisionnement}

Le graphique ci-dessous illustre les très forts échanges qui existent entre la France et l'Allemagne sur le marché de l'électricité. La courbe noire représente l'éolien en Allemagne, la courbe grise l'éolien et le photovoltaïque en Allemagne et les bâtonnets le solde des échanges commerciaux France-Allemagne. Ce graphique met en lumière l'influence des productions variables (photovoltaïque et éolienne) sur le sens des échanges.

\section{Graphique 1 : Évolution comparée du solde des échanges sur la frontière franco-allemande et de la production éolienne et photovoltaïque en Allemagne}

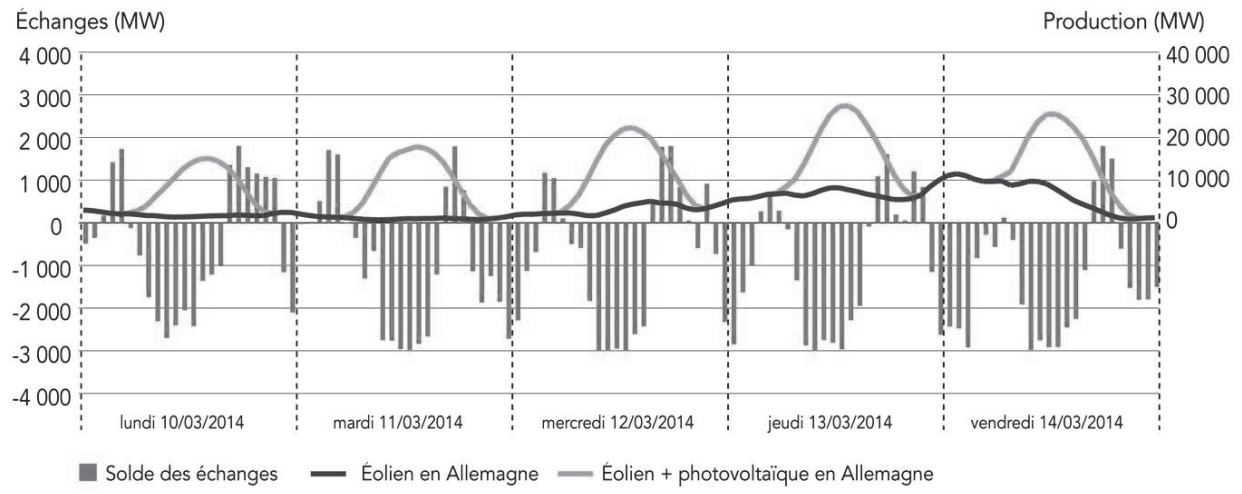

Source : RTE, Bilan électrique 2014, La Défense, 2015.

Il montre également que parmi les solutions qui permettront d'accompagner la transition énergétique figure en premier lieu le développement des interconnexions permettant de mutualiser les capacités de production européenne et de faire foisonner des gisements d'énergies renouvelables à une grande échelle. Dans ce contexte de transition énergétique, les réseaux énergétiques européens sont soumis à un cahier des charges profondément renouvelé. Ils devront permettre :

- de piloter une complexité d'un ordre nouveau, en raison de la variabilité de certaines énergies renouvelables, mais aussi avec l'émergence de nouveaux usages, tels que les véhicules électriques ;

- d'assurer les solidarités entre les États et les régions ;

- d'accompagner la décentralisation.

POUR CONCLURE, LA TRANSITION ÉNERGÉTIQUe CONDUIT À une localisation différente des sources de production. Les nouveaux sites, plus dispersés que les moyens de production «conventionnels », ne coïncident pas avec la cartographie antérieure. Les productions éolienne ou photovoltaïque sont généralement situées dans des zones éloignées des centres de consommation. Le réseau doit donc s'adapter rapidement pour être en mesure d'accueillir les nouvelles sources de production. 
Cela implique la création de nouveaux ouvrages, la modification des ouvrages existants, l'accueil au niveau local - dans le cadre de schémas régionaux - des énergies éolienne et photovoltaïque. Si jusqu'à présent le réseau électrique était construit pour répondre aux évolutions de la demande - caractérisée par une très forte thermosensibilité -, l'enjeu demain sera moins de suivre cette progression de la demande - qui devrait arriver à des niveaux de croissance différents - que d'accompagner l'insertion des nouvelles formes de production, ce qui induit un double challenge. Le premier est lié à la localisation : il s'agit d'aller sur le terrain dans des zones qui historiquement n'étaient pas desservies par le réseau de transport, ce qui rend nécessaire un travail de concertation pour pouvoir permettre l'acceptation des différents ouvrages impliqués. Le deuxième consiste à être en cohérence, en termes de dynamique, avec des modes de production qui changent significativement sur des durées relativement courtes (un an ou deux) alors que les infrastructures de réseau, du fait notamment des procédures administratives afférentes, requièrent souvent des délais très longs pour pouvoir être mises en œuvre. Par exemple, sur les grandes infrastructures de type $400 \mathrm{kV}$, les délais sont de l'ordre d'une décennie. Tel est donc le double défi à relever pour pouvoir pleinement accompagner la transition énergétique. 



\section{Transition énergétique en RFA : impact sur les réseaux électriques en Allemagne ${ }^{1}$}

\section{Ulrike HANSEN}

La réorientation de la transition énergétique allemande est en cours depuis le début de l'année 2014 (sur l'impératif de réformes, voir Bourgeois, 2014). Avec l'entrée en vigueur, le 1er août 2014, de la loi de révision de la loi de promotion des énergies renouvelables (Erneuerbare Energien-Gesetz, EEG), les mesures suivantes ont été mises en œuvre : définition d'objectifs de production d'énergie de source renouvelable, révision des critères de soutien aux énergies renouvelables et obligation de vente directe sur le marché de l'électricité. Par ailleurs, le ministère fédéral de l'Économie et de l'Énergie a publié le 24 juin 2014 un " Agenda énergie en 10 points » (10 Punkte-Energie-Agenda) qui vise la coordination des approches, et des calendriers afférents, à savoir : réforme de la loi EEG, mise en œuvre conjointe du paquet énergie-climat 2030 au sein de l'Union européenne, révision du système européen d'échange de quotas d'émission de $\mathrm{CO}_{2}$, nouvelle approche de l'organisation du marché de l'électricité et de coordination des mix énergétiques, définition d'une stratégie d'efficacité énergétique dans le BTP, développement du réseau allemand de transport et de distribution d'électricité, suivi annuel de l'avancée de la transition énergétique (monitoring) et coopération renforcée des plates-formes de recherche en la matière.

À la suite d'une consultation publique, le ministère fédéral de l'Économie et de l'Énergie a publié le livre blanc sur le développement du marché énergétique allemand Weißbuch "Ein Strommarkt für die Energiewende ») en 2015. Les principales idées du livre blanc ont été intégrées à la loi sur la conception du marché de l'électricité (Strommarktgesetz), laquelle a été adoptée par le Conseil des ministres le 4 novembre 2015 et par le Parlement allemand le 8 juillet 2016, et est entrée en vigueur le 30 juillet 2016. Les fondements principaux de la loi sont : la libre fixation des prix, une surveillance élaborée de la sécurité d'approvisionnement, le maintien d'une "réserve du réseau » (pour assurer la sécurité de l'exploitation du réseau et pour combler l'écart temporel du développement du réseau) et une "réserve de capacité » pour réagir aux situations extrêmes et imprévisibles du marché de l'énergie.

L'accélération du développement $d u$ réseau électrique est un élément central de la transition énergétique allemande depuis son lancement il y a plus de quinze ans. Après l'arrêt de huit réacteurs (7+1) intervenu en 2011 à la suite du moratoire de sortie accélérée du nucléaire, le volume d'électricité d'origine nucléaire injecté dans le réseau a baissé de quelque $9700 \mathrm{MW}$, ce qui a profondément modifié la structure du flux de charge, se traduisant par une hausse des importations d'électricité (cette situation s'est toutefois inversée entretemps, l'Allemagne étant aujourd'hui exportatrice) et des flux acheminés d'est en

\footnotetext{
${ }^{1}$ Version revue et actualisée en août 2016 d'un article paru dans la revue du CIRAC Regards sur l'économie allemande, $\mathrm{n}^{\circ} 114$, automne 2014 , $\mathrm{NdE}$.
} 
ouest. Cet impact du moratoire sur l'abandon du nucléaire d'ici 2022 à la fois sur les réseaux de transport et la sécurité de l'approvisionnement vient rappeler l'impératif du développement des réseaux.

\section{Le développement du marché de l'énergie en Allemagne}

Depuis plus d'une décennie, la question de l'extension des réseaux joue un rôle central dans la politique de transition énergétique allemande. Avant l'institution d'une régulation propre au marché de l'électricité en 2005 , le calcul prévisionnel des besoins de production, de la consommation et des impératifs de développement des réseaux s'effectuait au sein même de chaque entreprise du secteur de l'électricité ; ces électriciens étaient alors des sociétés verticalement intégrées. Or la libéralisation (1999) a eu pour effet la séparation des activités de production, de transport et de distribution (unbundling), de même que la multiplication des acteurs sur le marché de la production d'électricité. À cela est venu s'ajouter le choix d'un mix énergétique accordant une place croissante à l'électricité produite à partir de sources renouvelables. Il est alors apparu dès 2003 que cette évolution rendait incontournable un effort accru de développement du réseau à très haute tension et que celui-ci devait être accompagné par les pouvoirs publics. Comme l'actuelle autorité de régulation, la Bundesnetzagentur (BNetzA), n'avait alors pas encore de compétences énergétiques, c'est l'Agence allemande de l'énergie (Deutsche Energie-Agentur, dena) qui fut chargée par le ministère fédéral de l'Environnement de réaliser un rapport sur la planification des réseaux à l'horizon 2020, intégrant l'éolien onshore et offshore (rapport Energiewirtschaftliche Planung für die Netzintegration von Windenergie in Deutschland an Land und Offshore bis zum Jahr 2020, 2005).

\section{Le marché de l'électricité allemand - données clés}

En Allemagne, on compte quatre exploitants de réseaux de transport d'électricité : Amprion, Tennet, Transnet BW et 50 Hertz. Ces sociétés sont toutes de droit privé, au statut de SARL $(\mathrm{GmbH})$. Tous les opérateurs ont obtenu la certification délivrée par l'autorité de régulation, la Bundesnetzagentur, de même que l'opérateur de réseaux offshore Tennet Offshore mbH.

Le réseau de répartition d'électricité est géré actuellement par 884 sociétés.

La gestion des réseaux est totalement séparée des activités que sont la production d'électricité et sa distribution au consommateur final. La part de marché des quatre plus grandes sociétés de production d'électricité s'élève à environ $73 \%$ de la production nette d'électricité qui est de 408 TWh (état au 31 décembre 2014). Alors que la production d'électricité de source conventionnelle est tombée à 426,6 TWh en 2014 (soit -20,5 TWh par rapport à 2013), celle issue de sources renouvelables a progressé de 8,4 TWh pour s'établir à 154,8 TWh en 2014. L'Allemagne est exportateur net d'électricité et occupe, en sa qualité de pays de transit, le rôle de plaque tournante dans le système central de couplage des marchés européens (Bundesnetzagentur, Monitoringbericht, 2015).

\section{Le cadre législatif pour accélérer le développement des réseaux}

La loi sur l'approvisionnement en gaz et en électricité adoptée en 2005 et surnommée «loi sur l'économie de l'énergie » (Energiewirtschaftsgesetz, EnWG) fait obligation aux opérateurs de réseaux «d'entretenir et d'optimiser en fonc- 
tion des besoins, de renforcer et de développer le réseau dans la mesure de ce qui est économiquement raisonnable » (§ 11, al. 1 loi EnWG). Ils peuvent pour cela déposer auprès de la BNetzA des demandes de soutien à l'investissement pour des projets individuels de développement du réseau. La loi EnWG confie également aux exploitants de réseaux de transport la mission explicite de planifier le réseau de transport. Ceux-ci devaient déposer tous les deux ans devant la BNetzA un rapport sur l'état de leur planification. Depuis 2011, ce rapport devait être remis tous les ans. Cependant, l'intervalle de soumission du rapport été porté à deux ans à partir de 2016.

Or l'extension et la modernisation du réseau a pris beaucoup de retard, du fait entre autres de la lenteur des procédures administratives d'autorisation. Face à la persistance des lacunes et pénuries constatées, le législateur a donc dû prendre une série de mesures pour accélérer le développement du réseau.

Une première loi visant à accélérer les procédures de planification d'infrastructures a ainsi été adoptée le 9 décembre 2006. Entrée en vigueur le 17 décembre 2006, elle modifie les procédures administratives et réglementaires d'autorisation de certaines infrastructures de transport de gaz et d'électricité contenues dans la loi EnWG, importantes notamment pour sécuriser l'approvisionnement. Elle facilite les procédures réglementaires d'autorisation.

Une deuxième loi accélérant le développement des réseaux a été adoptée le 21 août 2009: la loi sur l'extension des lignes de transport d'énergie (Energieleitungsausbaugesetz, EnLAG). Une telle loi était devenue nécessaire parce que la précédente n'avait pas suffi à réduire les retards pris tant au niveau de la planification que de la réalisation de l'extension du réseau. Le pivot de la modification est donc l'inscription des différents projets - notamment des projets d'intérêt commun européen - au sein d'une loi de programmation prévisionnelle. Auparavant, le contrôle du bien-fondé de ces projets d'extension s'effectuait dans le cadre de plusieurs procédures administratives relevant de la compétence des Länder : la procédure d'aménagement et la procédure réglementaire d'autorisation permettant la construction des lignes, et sur l'aspect du financement, la procédure d'octroi des budgets d'investissement par la Bundesnetzagentur.

Enfin, la loi sur l'accélération du développement des réseaux (Netzausbaubeschleunigungsgesetz, NABEG) a été adoptée en 2011. Elle simplifie en profondeur les processus d'harmonisation des scénarios de planification des réseaux et les procédures d'autorisation (procédure d'approbation des grands projets publics) tout en en réduisant nettement la durée.

\section{1 : loi NABEG et simplification des procédures}

Depuis 2011, la planification des réseaux de transport d'électricité s'effectue de la manière suivante ( $\S 12 \mathrm{a}-12 \mathrm{e}$ EnWG) : les exploitants de réseaux de transport allemands présentent chaque année un schéma-cadre conjoint. Il constitue la base sur laquelle s'effectue la planification de l'ensemble des réseaux, qu'ils 
soient onshore ou offshore. Il comprend au moins trois projections (scénarios) censés correspondre aux évolutions probables des objectifs de politique énergétique fixés par le gouvernement fédéral au cours des dix années à venir. L'un des scénarios porte sur les deux décennies à venir. Les exploitants de réseaux de transport posent donc, pour chacun des scénarios, des hypothèses de travail en ce qui concerne la production, la distribution et la consommation d'électricité, de même que les échanges avec les États voisins, et ils prennent en considération également les programmes d'investissement concernant l'infrastructure des réseaux européens. Ce projet de schéma-cadre est remis à la Bundesnetzagentur qui le soumet à une procédure de consultation publique à l'issue de laquelle, et en tenant compte des avis émis lors de cette dernière, l'autorité de régulation approuve annuellement ce schéma-cadre. L'intervalle de proposition d'un schéma-cadre par les gestionnaires des réseaux de transport allemands a également été porté à deux ans à partir de 2016. Les différents scénarios sont désormais établis à un horizon allant de 10 à 15 ans à moyen terme et de 15 à 20 ans à long terme.

Tableau 1 : Objectifs du schéma-cadre approuvé par la Bundesnetzagentur le 19 décembre 2014 et relatif au projet de développement des réseaux 2025 ainsi qu'au projet de développement des réseaux offshore 2025

\begin{tabular}{|c|c|c|c|c|c|c|c|}
\hline & $\begin{array}{c}\text { Référence } \\
2013\end{array}$ & $\begin{array}{l}\text { Scénario } \\
\text { A } 2025\end{array}$ & $\begin{array}{l}\text { Scénario } \\
\text { B1 } 2025\end{array}$ & $\begin{array}{l}\text { Scénario } \\
\text { B1 } 2035\end{array}$ & $\begin{array}{l}\text { Scénario } \\
\text { B2 } 2025\end{array}$ & $\begin{array}{l}\text { Scénario } \\
\text { B2 } 2035\end{array}$ & $\begin{array}{c}\text { Scénario } \\
\text { C } 2025\end{array}$ \\
\hline Nucléaire & 12,1 & 0,0 & 0,0 & 0,0 & 0,0 & 0,0 & 0,0 \\
\hline Houille & 25,9 & 25,8 & 21,8 & 11,0 & 21,8 & 11,0 & 14,9 \\
\hline Gaz naturel & 26,7 & 26,5 & 29,9 & 40,7 & 29,9 & 40,7 & 29,5 \\
\hline Pétrole & 4,1 & 1,3 & 1,1 & 0,8 & 1,1 & 0,8 & 1,1 \\
\hline Autres sources conventionnelles & 4,7 & 3,2 & 3,1 & 3,1 & 3,1 & 3,1 & 3,1 \\
\hline Total prod. de source conventionnelle & 101,1 & 79,6 & 77,3 & 77,5 & 77,3 & 77,5 & 67,4 \\
\hline Éolien onshore & 33,8 & 53,0 & 63,8 & 88,8 & 63,8 & 88,8 & 59,0 \\
\hline Éolien offshore & 0,5 & 8,9 & 10,5 & 18,5 & 10,5 & 18,5 & 10,5 \\
\hline Photovoltaïque & 36,3 & 54,1 & 54,9 & 59,9 & 54,9 & 59,9 & 54,1 \\
\hline Biomasse & 6,2 & 6,4 & 7,4 & 8,4 & 7,4 & 8,4 & 6,4 \\
\hline Total production & 182,2 & 206,4 & 218,7 & 258,5 & 218,7 & 258,5 & 201,8 \\
\hline Consommation nette prévisible [TWh] ${ }^{*}$ ) & 543,6 & 543,6 & 543,6 & 543,6 & 543,6 & 543,6 & 516,4 \\
\hline Pic annuel $[G W]^{*}$ ) & 82,8 & 84,0 & 84,0 & 84,0 & 84,0 & 84,0 & 79,8 \\
\hline
\end{tabular}

Source : http://www.netzausbau.de/SharedDocs/Downloads/DE/2025/SR/Szenariorahmen_2025_Ge nehmigung.pdf [consulté le 26/07/2016]. *) Y inclus pertes en ligne dans le réseau de distribution.

Sur la base du schéma-cadre ainsi retenu, les exploitants de réseaux se mettent d'accord, chaque année, sur des projets de développement des réseaux (Netzentwicklungspläne, NEP), onshore et offshore (\$12b EnWG). Ces projets annuels doivent contenir toutes les mesures opportunes visant l'optimisation des réseaux selon les besoins, l'accroissement de leur capacité et leur extension. Celles-ci sont approuvées dès lors qu'elles sont nécessaires pour assurer le fonc- 
tionnement sûr et fiable des réseaux au cours des dix années à venir. Ces projets annuels sont eux aussi soumis à consultation publique avant d'être transmis pour approbation à l'autorité de régulation BNetzA.

Après examen, cette dernière les soumet à son tour à consultation. Afin de prendre en considération dès l'amont l'impact environnemental de ces projets, la BNetzA effectue un contrôle stratégique de conformité avec la protection de l'environnement du plan fédéral de programmation des besoins (Bundesbedarfsplan). Sont ainsi évaluées, pour chacun des projets, les répercussions sur la santé humaine, la biodiversité et l'environnement que pourrait avoir la construction de lignes électriques, qu'elles soient aériennes ou souterraines, qu'elles transportent du courant continu ou triphasé. Les conclusions de ces travaux sont ensuite résumées dans un rapport sur l'environnement soumis lui aussi à consultation publique. La BNetzA prend ensuite en considération les avis prononcés par les citoyens et les administrations lorsqu'elle entérine les projets de développement des réseaux onshore et offshore (NEP). Ceux-ci, de même que le rapport sur l'environnement, constituent les deux piliers sur la base desquels s'effectue la programmation annuelle du développement des réseaux.

\section{6 : changement de la périodicité de la planification des réseaux}

À partir de l'année 2016, la procédure du schéma-cadre alterne avec la planification de l'ensemble des réseaux tous les deux ans. Ainsi, le développement et la proposition d'un schéma-cadre sont obligatoires pour les gestionnaires des réseaux de transport pendant les années paires, tandis que l'approbation des NEP onshore et offshore aura lieu pendant les années impaires jusqu'au 31 décembre. Pendant les années impaires, les exploitants de réseaux présentent à la Bundesnetzagentur des rapports de mise en œuvre avant le 30 septembre (art. 2 de la première loi modifiant la loi sur l'obligation de mentionner les valeurs de consommation d'énergie sur l'étiquette des produits ainsi que les autres dispositions réglementaires dans le domaine de l'énergie ${ }^{2}$ ).

En outre, si des modifications conséquentes sont intervenues, mais au minimum tous les trois ans, l'autorité de régulation soumet au gouvernement fédéral les NEP qu'elle a entérinés et son rapport environnemental pour alimenter le projet de plan fédéral de programmation des besoins que le gouvernement fédéral soumet au vote du Parlement tous les trois ans au minimum également. C'est ainsi que prend forme la loi de programmation fédérale des besoins (Bundesbedarfsplangesetz, BBPIG).

\footnotetext{
${ }^{2}$ Erstes Gesetz zur Änderung des Energieverbrauchskennzeichnungsgesetzes (EnVKG) und zur Änderung weiterer Bestimmungen des Energiewirtschaftsrechts.
} 


\section{Carte 1 :}

\section{Lignes prévues dans le cadre de la loi de programmation fédérale des besoins 2015}

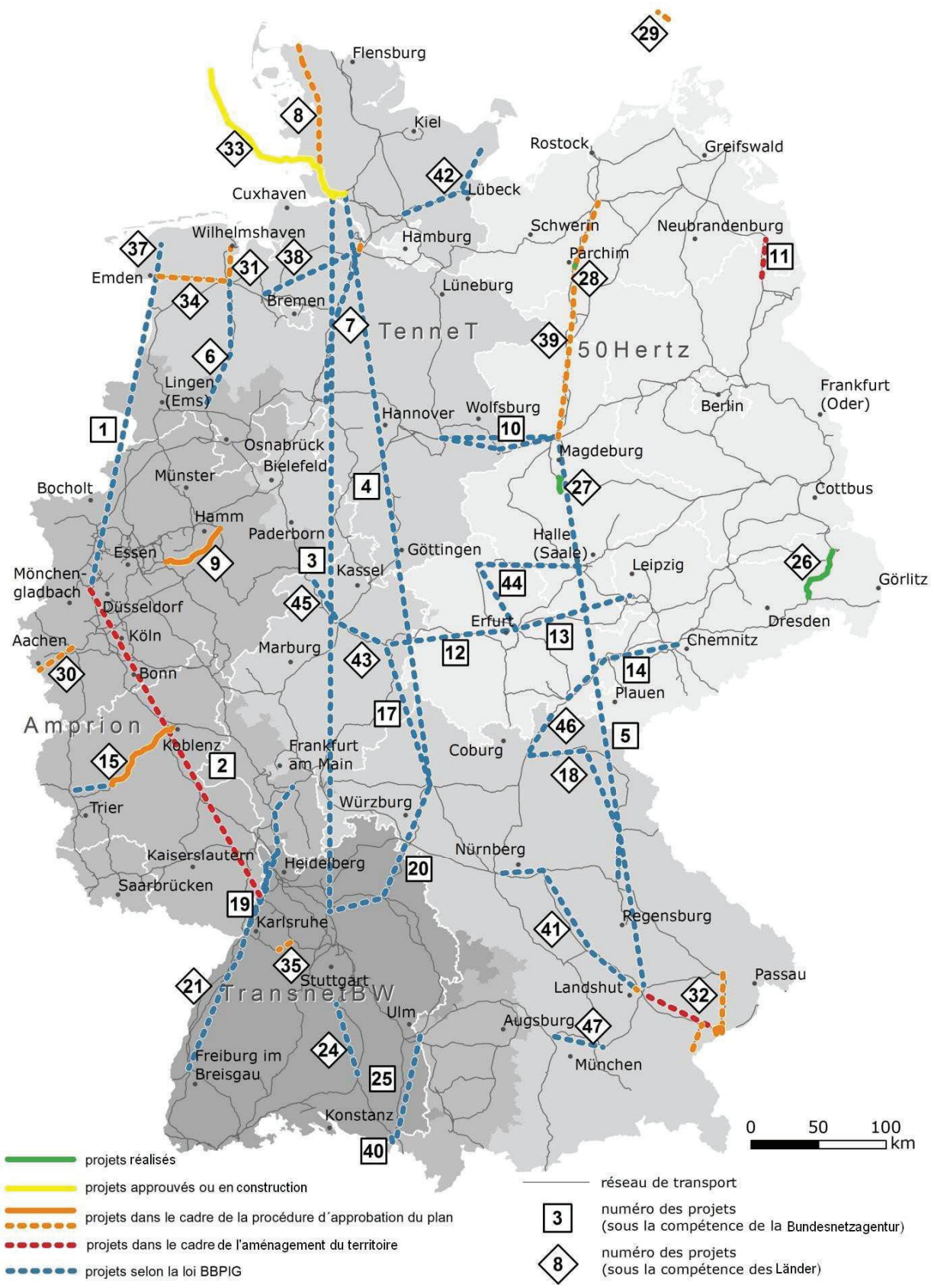

Source : http://www.netzausbau.de/leitungsvorhaben/de.html?cms_map=2 [consulté le 26/07/2016]. 
La loi de programmation fédérale des besoins, adoptée le 23 juillet 2013 pour la première fois et actualisée en 2015, a défini, sous l'angle des impératifs de politique énergétique, la nécessité du développement des réseaux et les besoins afférents tels que prévus par la loi, les rendant de la sorte contraignants pour les exploitants de réseaux, y compris dans le cadre de la procédure réglementaire d'autorisation (§§ 43-43d EnWG et §§ 18-24 NABEG).

Ces dispositions suppriment tout risque d'incertitude lié à l'appréciation de l'autorité de régulation, qu'il s'agisse de l'agrément des coûts programmés pour les travaux d'extension du réseau ou de l'octroi des autorisations afférentes. Non contentes de cela, elles font obligation aux exploitants de réseaux de transport de réaliser effectivement les projets d'extension et d'aménagement contenus dans les projets de développement approuvés par la BNetzA. La loi de programmation des besoins 2013-2015 apporte ainsi aux exploitants de réseaux une sécurité juridique maximale en ce qui concerne les investissements requis.

La loi de programmation fédérale des besoins a donc été amendée le 30 décembre 2015. Cette mise à jour, entrée en vigueur le 31 décembre 2015, tient compte de la planification des réseaux à l'horizon 2024. Pour certains projets, la nécessité de mise en œuvre ne s'est pas confirmée ou les coordonnées des mesures ont été changées. Par ailleurs, 11 projets ont été ajoutés.

De même, les règles juridiques ont subi une révision pour faciliter l'installation des lignes souterraines sous certaines conditions (loi du 30 décembre 2015 modifiant les dispositions réglementaires concernant la construction des lignes de transport d'énergie ${ }^{3}$ ).

Les lignes pour le transport de courant continu à très haute tension (high voltage direct current, HVDC) peuvent être installées en priorité en tant que lignes souterraines. Dans la version actualisée de la loi de programmation fédérale des besoins, trois projets HVDC ont été approuvés, leur mise en œuvre souterraine devenant possible selon cette nouvelle réglementation. Tous les corridors marqués par la lettre $\mathrm{E}$ dans l'annexe de la loi du 31 décembre 2015 (les corridors nommés A1, D, C1, C2 ainsi que le projet Oberzier-Belgique (P 65) dans l'approbation des projets de développement des réseaux (NEP) $2024^{4}$ ) pourront être réalisés en souterrain, à l'exception du corridor Ultranet (nommé A2 dans l'approbation des projets de développement des réseaux (NEP) 2024), pour lequel une priorité de mise en œuvre souterraine n'est pas prévue par la loi.

Toutefois, les lignes qui servent au transport de courant alternatif (alternating current, $\mathrm{AC}$ ) - et qui représentent la plupart des projets - continueront à être installées en version aérienne. Il est prévu de lancer des projets pilotes sou-

\footnotetext{
${ }^{3}$ Gesetz zur Änderung von Bestimmungen des Rechts des Energieleitungsbaus.

${ }^{4}$ Bundesnetzagentur, Bestätigung Netzentwicklungsplan Strom (Zieljahr 2024) [en ligne], Bundesnetzagentur, Bonn, 4 septembre 2015, p. 7. Disponible sur : http://data.netzausbau.de/2024/NEP/NEP2024_Bestaetigung. pdf [consulté le 02/08/2016].
} 
terrains pour certains projets inscrits dans la loi, de façon à approfondir les expériences et à améliorer leur développement technique.

Dans un deuxième temps, les exploitants de réseaux de transport peuvent faire auprès de l'autorité de régulation une demande d'attribution de corridors à l'intérieur des tracés programmés par la loi fédérale de programmation des besoins. Cette procédure, dite de planification technique fédérale (Bundesfachplanung), s'applique aux liaisons à très haute tension traversant les frontières des Länder ou des États limitrophes. Ces corridors constituent alors la base de la procédure réglementaire d'autorisation pour la construction des lignes, prévue par la procédure de planification des réseaux confiée notamment à la BNetzA $(\$ \S 4 s q$. NABEG). Ces deux procédures - planification technique fédérale et procédure réglementaire d'autorisation - incluent elles aussi une large consultation du public.

Une consultation d'une telle ampleur a pour objectif foncier d'engager le dialogue avec toutes les parties intéressées et tous les citoyens concernés et ce, le plus tôt possible afin de pouvoir rendre intelligibles les projets d'infrastructures, de permettre à l'opinion d'exprimer ses craintes et d'en débattre. C'est ainsi que, depuis 2011, l'autorité de régulation organise, dans l'ensemble de l'Allemagne, nombre de manifestations destinées à informer les citoyens sur les schémas-cadre, les projets de développement des réseaux et les rapports environnementaux, ainsi que des débats sur les technologies, la protection de l'environnement ou l'état de la recherche.

\section{Impact sur les réseaux : la question de la sécurité de l'approvisionnement}

En plus de la planification de l'extension des réseaux et de la simplification des procédures réglementaires d'autorisation, une autre question encore est centrale pour le bon déroulement de la transition énergétique : celle de la sécurité de l'approvisionnement. L'abandon progressif de la production d'électricité de source nucléaire exige en effet que soit préservée une capacité de production suffisante pour couvrir les besoins en électricité. Il implique également que soit garantie la stabilité du réseau lorsque, du fait de la volatilité de la production des énergies renouvelables, survient un déséquilibre entre production injectée et énergie soutirée. Et que, enfin, les réseaux soient sécurisés contre l'intervention de tiers. Pour répondre à ces exigences, les exploitants de réseaux de transport et l'autorité de régulation veillent à garantir la capacité de production de source conventionnelle nécessaire pour les années à venir.

Cette mission s'effectue d'une part via un suivi de l'arrêt des diverses centrales. À cet effet, les exploitants de sites de production et de stockage sont tenus de notifier aux exploitants de réseaux de transport concernés et à la BNetzA leur projet de fermeture au moins 12 mois avant l'arrêt de leurs installations. Celle-ci n'intervient pas nécessairement à la date théoriquement prévue. Car si l'exploitant du réseau de transport concerné justifie de l'importance pour le système du site à fermer et que l'autorité de régulation l'agrée (\$ $13 \mathrm{a}$, al. 2 
EnWG), la fermeture est ajournée. Une centrale ou un site sont jugés importants pour le système lorsque l'arrêt définitif de la centrale ou du site en question entraînerait une mise en danger ou une perturbation non négligeables de la sécurité ou de la fiabilité du système d'approvisionnement en électricité, et qu'il ne pourrait y être remédié d'aucune manière. Si la BNetzA donne son agrément à la catégorisation ainsi effectuée par l'exploitant du réseau de transport, la fermeture du site en question est repoussée, de 24 mois au maximum.

D'autre part, dans l'objectif d'assurer la stabilité du réseau, les exploitants de réseaux de transport doivent évaluer les besoins prévisionnels en matière de centrales de réserve et faire valider leurs prévisions par la BNetzA. La capacité de production ainsi mise en réserve est destinée à pallier une surcharge éventuelle du réseau. Il s'agit là d'une mesure transitoire destinée à assurer la sécurité de l'approvisionnement tant que le réseau électrique, encore insuffisamment développé, est fragilisé par l'injection accrue d'électricité de source renouvelable (volatilité) et l'arrêt progressif de centrales conventionnelles. Les centrales de réserve représentent ainsi une assurance supplémentaire pour la stabilisation du réseau dans le cas où est épuisé le potentiel de mesures de redispatching de l'ensemble des centrales existant sur le marché. Ces mesures ont pour but d'éviter une surcharge possible d'une partie du réseau : si une congestion est prévisible dans un segment précis du réseau, les centrales en amont de ce segment sont tenues d'injecter moins d'électricité dans le réseau et celles situées en aval d'augmenter la puissance injectée. De cette manière (redispatching) est créé un flux de charge qui élimine la congestion.

La nécessité des mesures de redispatching et des mesures de gestion d'injection d'électricité des énergies renouvelables dans le réseau s'est considérablement accrue ces dernières années. Pour le redispatching, la durée des interventions a quintuplé entre 2010 (avec 1588 heures par an) et 2014 (avec 8453 heures par an) et presque décuplé entre 2010 et 2015 (avec 15811 heures par an). La Bundesnetzagentur collecte des données et publie des évaluations trimestrielles depuis $2015^{5}$.

Les dernières prévisions des exploitants de réseaux, validées le 4 mai 2015 par la BNetzA, sont publiées en ligne : "Pour l'hiver 2015-2016, la puissance de production nécessaire à fournir par les centrales de réserve est de $6700 \mathrm{MW}$ à $7800 \mathrm{MW}$, dont un volume de $489 \mathrm{MW}$ jusqu'à $1388 \mathrm{MW}$ maximum doit encore être couvert. Pour la période 2016-2017, elle est de $6600 \mathrm{MW}$ à $7700 \mathrm{MW}$, dont un volume de $3198 \mathrm{MW}$ jusqu'à $4212 \mathrm{MW}$ maximum doit encore être couvert. Puis pour la période 2018-2019, le volume est de $1600 \mathrm{MW}$. » Une part importante de cette puissance de production est d'ores et déjà garantie par le biais d'engagements contractuels ou d'obligations légales.

\footnotetext{
5 Bundesnetzagentur, Netz- und Systemsicherheit [en ligne], Bonn, 2016. Disponible sur : http://www.bundesnetzagentur.de/cln_1432/DE/Sachgebiete/ElektrizitaetundGas/Unternehmen_Institutionen/ Versorgungssicherheit/Stromnetze/Netz_Systemsicherheit/Netz_Systemsicherheit_node.html [consulté le 04/08/2016].
} 
$\mathrm{Au}$ vu du potentiel du marché des centrales de production en Allemagne comme dans les États limitrophes dans l'Union européenne, la puissance manquante devrait être couverte sans difficulté.

TOUS CES ÉLÉMENTS PERMETTENT DE MIEUX MESURER l'ampleur de l'impact de la transition énergétique allemande et de comprendre le rôle crucial que joue dans ce contexte le développement des réseaux électriques en Allemagne, mais également en Europe. Quant au fait qu'il soit possible de trouver hors des frontières allemandes des centrales de réserve et de conclure des contrats d'approvisionnement avec elles, il révèle la dimension européenne de la transition énergétique allemande.

\section{Traduction d'Isabelle Bourgeois et d'Ulrike Hansen}

\section{Indications bibliographiques}

[S.A.], Bundesbedarfsplangesetz du 23 juillet 2013 (BGB1. I 2013 p. 2543; 2014 I p. 148), modifiée en dernier lieu par l'article 7 du 21 décembre 2015 (BGB1. I 2015 p. 2495-2497)

[S.A.], Energieleitungsausbaugesetz du 21 août 2009 (BGB1. I p. 2870)

[S.A.], Erstes Gesetz zur Änderung des Energieverbrauchskennzeichnungsgesetzes und zur Änderung weiterer Bestimmungen des Energiewirtschaftsrechts (BGB1 I 2015 p. 2140, 10 décembre 2015)

[S.A.], Gesetz zur Änderung von Bestimmungen des Rechts des Energieleitungsbaus du 21 décembre 2015 (BGB1. I 2015 p. 2490)

BouRgeOIS I., «La transition énergétique allemande : mission impossible ? », Regards sur l'économie allemande, $\mathrm{n}^{\circ} 112,2014$, p. 5-16

BUNDESMINISTERIUM FÜR WIRTSCHAFT UND ENERgIE (BMWI), 10 Punkte Energie Agenda [en ligne], Berlin, 26 juin 2014. Disponible sur: http://www.bmwi.de/BMWi/Redaktion/PDF/0-9/10-punkte-energieagenda,property=pdf,bereich=bmwi2012, sprache=de,rwb=true.pdf [consulté le 24/10/2016]

BUNDESMINISTERIUM FÜR WIRTSCHAFT UND ENERGIE (BMWI), Reform des Erneuerbare-EnergienGesetzes beschlossen [en ligne], Berlin, 27 juin 2014. Disponible sur : http://www.bmwi.de/DE/Themen/ energie,did=644468.html [consulté le 24/10/2016]

BUNDESMINISTERIUM FÜR WIRTSCHAFT UND ENERGIE (BMWI), Weißbuch „Ein Strommarkt für die Energiewende, [en ligne], Berlin, juillet 2015. Disponible sur: http://www.bmwi.de/DE/Themen/ Energie/Strommarkt-der-Zukunft/Strommarkt-2-0/stellungnahmen-weissbuch,did=718200.html [consulté le 24/10/2016]

BundeSnetZaGentur, Monitoringbericht 2007 [en ligne], Bonn, 29 juin 2007. Disponible sur : http://www.bundesnetzagentur.de/SharedDocs/Downloads/DE/Sachgebiete/Energie/Unternehmen_Institution en/DatenaustauschUndMonitoring/Monitoring/Monitoringbericht2007.pdf?_blob=publicationFile\&v=2 [consulté le 24/10/2016]

BUNDESNETZAGENTUR, Bericht gemäß $\S 63$ Abs. $4 a$ EnWG zu Auswertung der Netzzustands- und Netzausbauberichte der deutschen Elektrizitätsübertragungsnetzbetreiber [en ligne], Bonn, 8 janvier 2008. Disponible sur: https://www.bundesnetzagentur.de/SharedDocs/Downloads/DE/Allgemeines/Bundesnetzagentur/ Publikationen/Berichte/2008/BerichtNetzzustandNetzausbauId12385pdf.pdf?_blob=publicationFile\&v=2 [consulté le 24/10/2016] 
Bundesnetzagentur, Monitoringbericht 2009 [en ligne], Bonn, 2009. Disponible sur : http://www.bundes netzagentur.de/SharedDocs/Downloads/DE/Sachgebiete/Energie/Unternehmen_Institutionen/Datenaustausch UndMonitoring/Monitoring/Monitoringbericht2009.pdf?_blob=publicationFile\&v=2 [consulté le 24/10/2016]

BUNDESNETZAGENTUR, Auswirkungen des Kernkraftwerk-Moratoriums auf die Übertragungsnetze und die Versorgungssicherheit : Bericht der Bundesnetzagentur an das Bundesministerium für Wirtschaft und Technologie [en ligne], Bonn, 11 avril 2011. Disponible sur : http://www.bmwi.de/BMWi/Redaktion/PDF/Publika tionen/auswirkungen-kernkraftwerk-moratorium-auf-uebertragungsnetze-und-versorgungssicherheit,property=pdf, ber\%20eich=bmwi2012,sprache=de,rwb=true.pdf [consulté le 24/10/2016]

BUNDESNETZAGENTUR, Genehmigung des Szenariorahmens für den Netzentwicklungsplan und den OffshoreNetzentwicklungsplan 2025 [en ligne], Bonn, 19 décembre 2014. Disponible sur: http://www.netzaus bau.de/SharedDocs/Downloads/DE/2025/SR/Szenariorahmen_2025_Genehmigung.pdf?_blob=publicationFi le [consulté le 24/10/2016]

BUNDESNETZAGENTUR, Bestätigung des Reservekraftwerksbedarfs : Ausgabejahr 2015 [en ligne], Bonn, 4 mai 2015. Disponible sur: https://www.bundesnetzagentur.de/SharedDocs/Pressemitteilungen/DE/2015/ 150504_ReserveStrom.html [consulté le 24/10/2016]

BundeSnetZagentur, Bestätigung Netzentwicklungsplan Strom (Zieljahr 2024) [en ligne], Bonn, 4 septembre 2015, p. 7. Disponible sur : http://data.netzausbau.de/2024/NEP/NEP2024_Bestaetigung.pdf [consulté le $24 / 10 / 2016]$

BundeSnetzagentur, Monitoringbericht 2015 [en ligne], Bonn, 10 novembre 2015. Disponible sur: http://www.bundesnetzagentur.de/SharedDocs/Downloads/DE/Allgemeines/Bundesnetzagentur/Publikationen /Berichte/2015/Monitoringbericht_2015_BA.pdf?_blob=publicationFile\&v=3 [consulté le 24/10/2016]

BUNDESNETZAGENTUR, Netz- und Systemsicherheit [en ligne], Bonn, 2016. Disponible sur : http://www.bundesnetzagentur.de/cln_1432/DE/Sachgebiete/ElektrizitaetundGas/Unternehmen_Institutionen/ Versorgungssicherheit/Stromnetze/Netz_Systemsicherheit/Netz_Systemsicherheit_node.html [consulté le 24/10/2016]. 



\section{Enjeux de la transformation des systèmes électriques allemand et français à l'horizon $2030^{1}$}

\section{Dimitri PESCIA}

Les énergies éolienne et photovoltaïque sont amenées à jouer un rôle majeur dans la transformation des systèmes électriques, en Allemagne comme en France. Ces technologies sont désormais compétitives avec les centrales thermiques conventionnelles pour les nouveaux investissements. Leurs caractéristiques intrinsèques (variabilité, coûts d'investissements élevés et coûts marginaux quasi nuls) bouleversent néanmoins profondément le fonctionnement des systèmes et marchés électriques, qui doivent évoluer vers davantage de flexibilité. En parallèle, des conditions d'investissement stables en faveur des énergies renouvelables doivent être préservées, afin de limiter leurs coûts et d'accompagner leur déploiement au rythme souhaité. En matière de sécurité d'approvisionnement, la France et l'Allemagne poursuivent aujourd'hui une approche différente : introduction d'un marché de capacité en France, réserve stratégique opérée hors marché en Allemagne. Par ailleurs, l'Allemagne et la France doivent relever des défis structurels essentiels, mais différenciés, relatifs à l'avenir de leur parc de production conventionnel : charbon pour l'Allemagne, nucléaire pour la France. Une réforme du marché des émissions de $\mathrm{CO}_{2}$ ainsi qu'un plan de sortie du charbon à long terme sont nécessaires pour permettre à l'Allemagne d'atteindre ses objectifs de baisse des émissions de $\mathrm{CO}_{2}$. La France est confrontée à des choix politiques et économiques majeurs en ce qui concerne le rééquilibrage de son mix électrique et la place qu'y jouera le nucléaire à long terme.

\section{L'Allemagne et la France au cœur de la transformation} du système électrique européen

La stratégie européenne bas carbone vise un développement conséquent des énergies renouvelables dans l'ensemble des secteurs énergétiques, et en particulier dans le secteur de la production d'électricité. Conformément à l'accord du Conseil européen d'octobre 2014, l'Union européenne s'est ainsi fixé un objectif de $27 \%$ d'énergie renouvelable dans sa consommation finale d'énergie d'ici 2030, ce qui correspond à une part d'environ $50 \%$ dans le secteur électrique (contre 28,6\% en 2015).

La France et l'Allemagne ont adopté des objectifs nationaux ambitieux, en ligne avec ces engagements européens. Dans le cadre de l'Energiewende, l'Allemagne vise ainsi $50 \%$ d'électricité renouvelable en 2030 (contre 32,5\% en 2015). La loi de transition énergétique française vise, quant à elle, un objectif de $40 \%$ d'électricité renouvelable au même horizon de temps (contre $18,7 \%$ en 2015).

\footnotetext{
${ }^{1}$ Article rédigé en mars 2016, NdE.
} 


\section{Carte 1 : Contribution des énergies renouvelables dans le mix électrique européen en 2030}

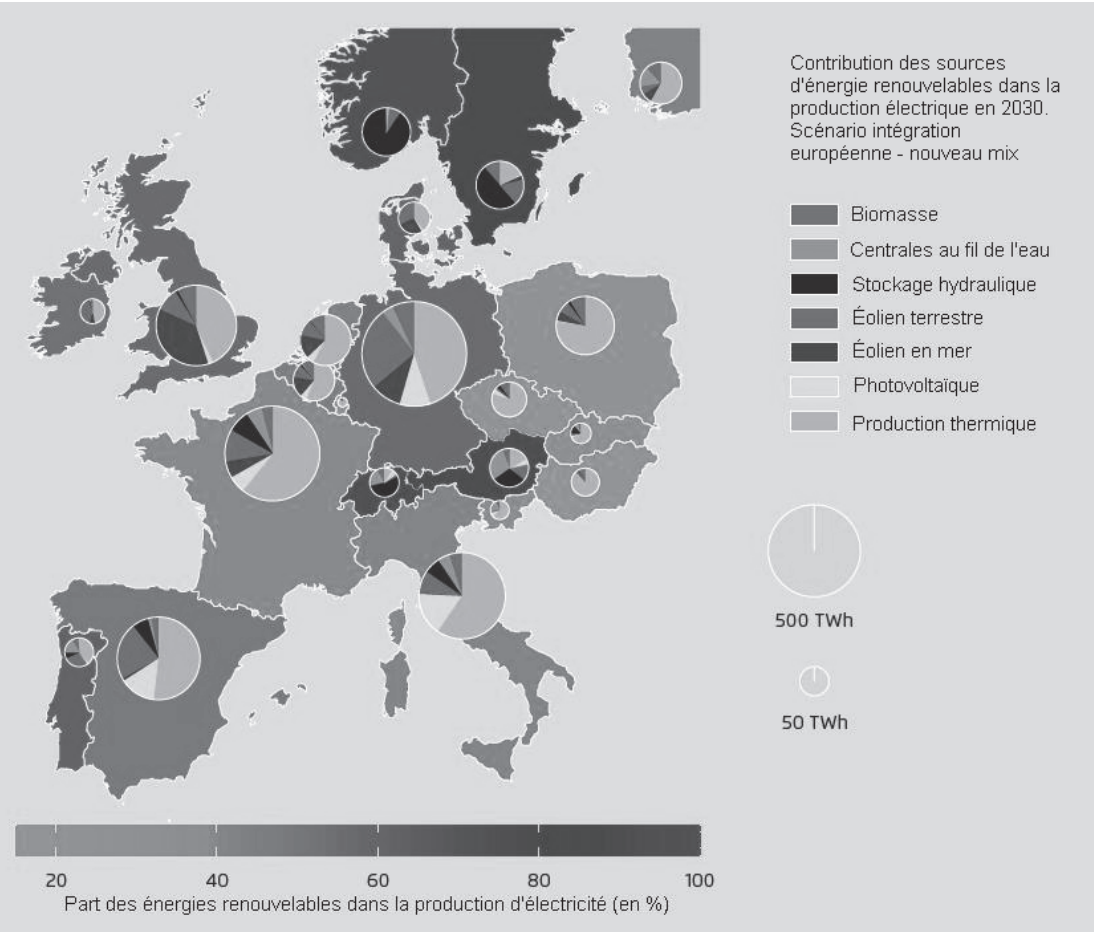

Source : Fraunhofer IWES (2015) sur la base d'ENTSO-E (2014) et des stratégies nationales.

La France et l'Allemagne jouent un rôle de premier plan dans la transformation des systèmes électriques européens, étant donné la taille de leurs marchés et leur niveau d'interconnexion élevé. Les marchés électriques allemand et français couvrent ensemble près d'un tiers de la production électrique européenne (647 TWh produits par l'Allemagne en 2015 et 546 TWh produits par la France). Par ailleurs, les deux États sont les deux premiers exportateurs d'électricité d'Europe en 2015, avec un solde exportateur net de 62 TWh pour la France (soit $11 \%$ de la production nationale) et $50 \mathrm{TWh}^{2}$ pour l'Allemagne ( $8 \%$ de la production nationale). Cette situation est la conséquence de l'important niveau d'interconnexion dont bénéficient les deux États (environ $21 \mathrm{GW}$ d'interconnexions à l'export pour l'Allemagne et $17 \mathrm{GW}$ pour la France ${ }^{3}$ ) et de la compétitivité de leur mix de production électrique.

\footnotetext{
${ }^{2}$ Bilan total des échanges physiques d'électricité. Selon ENTSO-E, le bilan total des échanges commerciaux atteint même 61 TWh en 2015.

${ }^{3}$ RTE (2016) ; Agora Energiewende (2016a).
} 


\section{L'éolien et le solaire photovoltaïque constituent les piliers de la transition énergétique}

Les énergies éolienne et solaire photovoltaïque sont amenées à jouer un rôle majeur dans l'atteinte des objectifs de décarbonation du système électrique européen. Après une baisse continue de leurs coûts de production, ces technologies sont aujourd'hui compétitives avec les énergies fossiles pour les nouveaux investissements (voir graphique 1). En Allemagne, le coût moyen de production de l'électricité (LCOE) de l'éolien terrestre atteint entre 6 et 9 centimes $/ \mathrm{kWh}$, en fonction des conditions de vent. Ce coût de production atteint entre 8 et 9 centimes/kWh pour le solaire photovoltaïque au sol dans le sud du pays. Ces niveaux sont similaires aux coûts de production des nouvelles centrales à gaz ou à charbon et inférieurs aux coûts des autres énergies décarbonées. Par ailleurs, ces coûts devraient continuer à baisser, en particulier pour les grandes centrales solaires photovoltaïques, atteignant en 2025 entre 4 centimes $/ \mathrm{kWh}$, dans le sud de l'Europe, et 6 centimes $/ \mathrm{kWh}$, dans les pays à l'ensoleillement comparable à celui de la France ${ }^{4}$.

En ce qui concerne le nucléaire, la situation est contrastée. En France, la prolongation de la durée de vie d'exploitation de certaines centrales nucléaires pourrait transitoirement s'avérer une option économiquement pertinente. Ces coûts de prolongation sont néanmoins encore relativement incertains et augmenteront si la production nucléaire diminue (en TWh) à capacité installée constante $(\mathrm{GW})$. En revanche, les coûts du nouveau nucléaire en Europe, par exemple ceux du projet d'Hinkley Point C au Royaume-Uni, s'avèrent aujourd'hui sans ambiguïté largement supérieurs à ceux des projets éoliens et photovoltaïques.

\section{Graphique 1 : Niveau des coûts de production moyens d'électricité annualisés (LCOE) de diverses technologies en 2015}

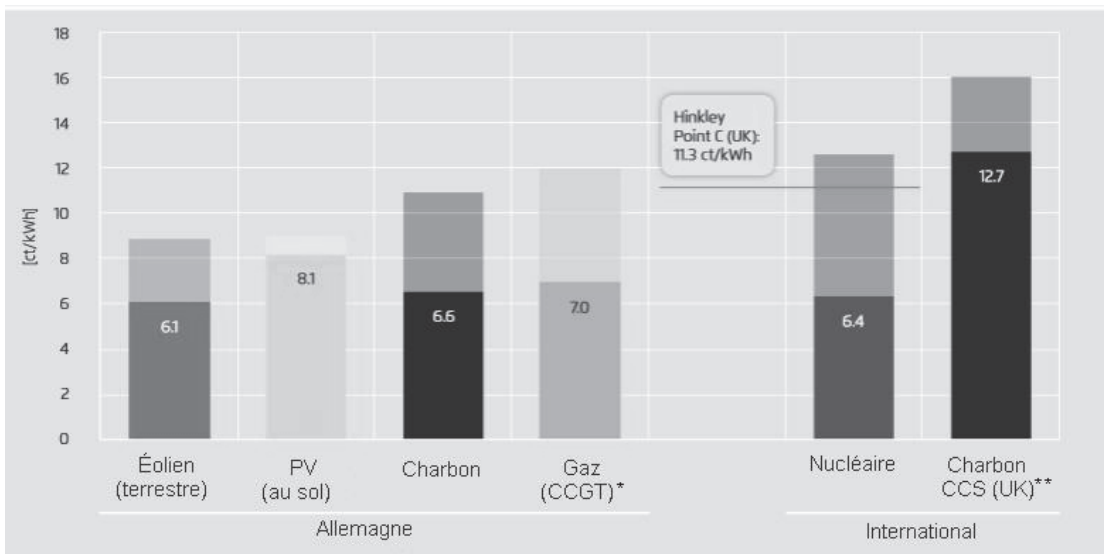

Source : Agora Energiewende (2015a). Les écarts sont la conséquence de diverses hypothèses relatives au prix du $\mathrm{CO}_{2}$, aux coûts d'investissement et aux taux d'utilisation des centrales. * CCGT : combined cycle gas turbine; $* *$ CCS : carbon capture and storage.

\footnotetext{
${ }^{4}$ Fraunhofer ISE (2015).
} 
L'éolien et le solaire photovoltaïque ne se cantonnent donc plus à un marché de niche, mais constituent les solutions décarbonées les plus compétitives pour les nouveaux investissements. Fin 2015, ces deux technologies représentaient déjà environ $85 \mathrm{GW}$ installés en Allemagne, soit davantage que la demande de pointe hivernale (environ $82 \mathrm{GW}$ ). Le pays vise un développement annuel supplémentaire d'au moins $5 \mathrm{GW}$ d'éolien terrestre et de photovoltaïque dans les années à venir. Par ailleurs, il vise $15 \mathrm{GW}$ d'éolien offshore installé d'ici 2030 (contre 3,5 GW aujourd'hui). Le marché français reste plus modeste, avec 16,5 GW d'éolien et de photovoltaïque installés fin 2015, mais les objectifs de croissance, tels que suggérés par les travaux de la programmation pluriannuelle de l'énergie, pourraient porter les capacités installées à 46 GW fin 2023, soit une croissance annuelle de 3,7 GW.

Les éoliennes et les centrales photovoltaïques possèdent néanmoins des caractéristiques spécifiques qui transforment profondément les systèmes et marchés électriques : les installations produisent de l'électricité uniquement lorsque le soleil brille et que le vent souffle et ne peuvent pas être déclenchées à la demande. Par ailleurs elles sont souvent installées loin des centres de forte consommation, ce qui peut nécessiter le développement de nouvelles infrastructures de réseaux. Dans ce contexte, le terme de coûts d'intégration est parfois proposé afin de comparer les technologies entre elles dans une approche davantage systémique.

\section{Graphique 2 : Illustration des diverses composantes des « coûts d'intégration »}

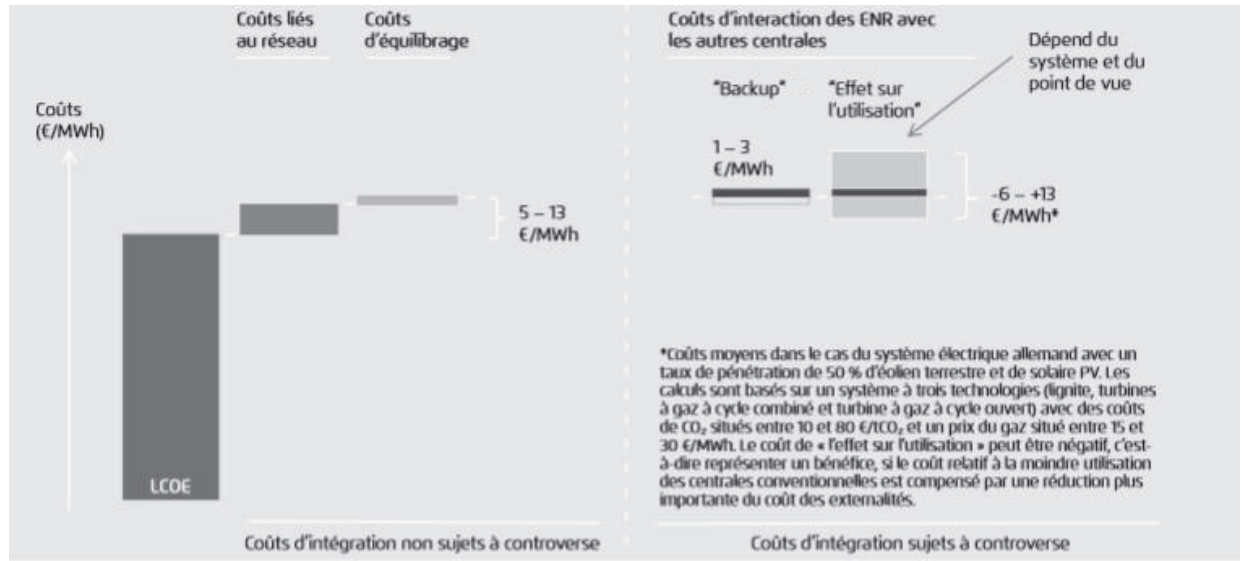

Source : Agora Energiewende (2015b).

Ces coûts d'intégration recouvrent typiquement les coûts de réseaux, les coûts d'équilibrage et le coût de l'impact des énergies renouvelables sur l'utilisation des centrales thermiques conventionnelles. Ces coûts dépendent de la flexibilité globale du système électrique, ainsi que du niveau d'internalisation du coût du $\mathrm{CO}_{2}$. Dans le cas du système électrique allemand avec un taux de pénétration de $50 \%$ d'énergies renouvelables variables, ces coûts d'intégration sont évalués à environ 
0,5 à 2,0 centimes $/ \mathrm{kWh}^{5}$. Ces coûts ne modifient donc pas fondamentalement la compétitivité des énergies renouvelables par rapport aux autres technologies.

\section{La flexibilité comme nouveau paradigme de la transformation des systèmes électriques}

La variabilité de la production d'électricité renouvelable contraint l'ensemble du système électrique à s'adapter, afin de répondre de manière davantage flexible à ces fluctuations. De nombreuses solutions techniques existent pour renforcer cette flexibilité.

Le renforcement des réseaux électriques nationaux et transnationaux constitue l'option de flexibilité la moins coûteuse. Les réseaux permettent de mutualiser, sur une maille géographique large, les divers moyens de production et les différents profils de consommation. Cette régionalisation apporte des bénéfices globaux, car les régimes de production d'électricité d'origine renouvelable ne sont pas parfaitement corrélés entre les pays. Par exemple, le vent ne souffle pas uniformément et simultanément sur toute l'Europe. Ces effets de foisonnement s'observent également pour la demande électrique, bien qu'ils soient moins prononcés ${ }^{6}$. La mise en réseau réduit donc la volatilité globale du système, limite le besoin de flexibilité résiduel et réduit l'écrêtement de la production renouvelable.

\section{Graphique 3 : Production éolienne à divers niveaux d'agrégation en Allemagne durant le mois de mai 2030}

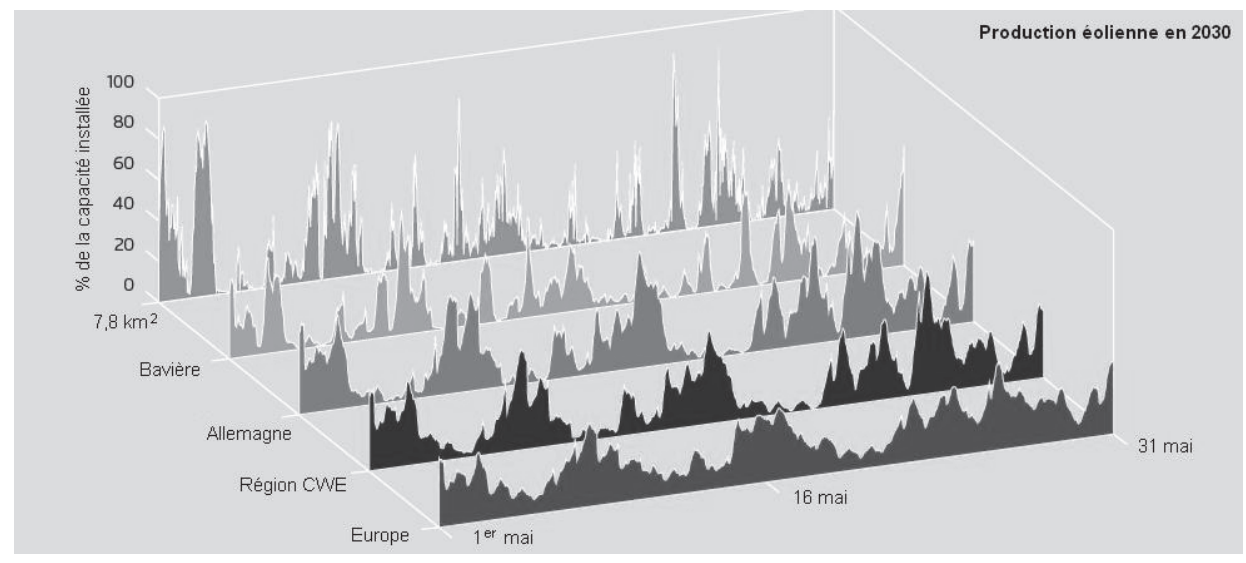

Source : Fraunhofer IWES (2015). Le profil de production éolien devient moins fluctuant à mesure que la maille géographique s'élargit.

\footnotetext{
${ }^{5}$ Agora Energiewende (2015b).

${ }^{6}$ Les pics de consommation se produisent dans tous les pays européens en général l'hiver entre $17 \mathrm{~h}$ et $20 \mathrm{~h}$. Les bilans prévisionnels d'adéquation montrent néanmoins que le pic de consommation à la maille régionale est inférieur de plusieurs GW à la somme des pics nationaux. Des travaux menés pour l'Allemagne et ses voisins ont quantifié cette différence à au moins 10 GW en 2015 et 20 GW en 2020 (Consentec, Fraunhofer ISI, r2b, 2015).
} 
La flexibilisation de la production électrique conventionnelle constitue la seconde option de flexibilité. Les centrales thermiques conventionnelles (gaz, charbon, nucléaire) doivent devenir des partenaires flexibles de la production renouvelable et réagir rapidement à leur variabilité. D'ici 2030, le nombre de centrales électriques de la région du Centre-Ouest de l'Europe ${ }^{7}$ opérant plus de 7000 heures par an, c'est-à-dire en mode dit «baseload», sera réduit d'environ $50 \%$.

\section{Graphique 4 : Production et consommation d'électricité dans la région du Centre-Ouest de l'Europe (CWE) durant une semaine de fin d'été 2030}

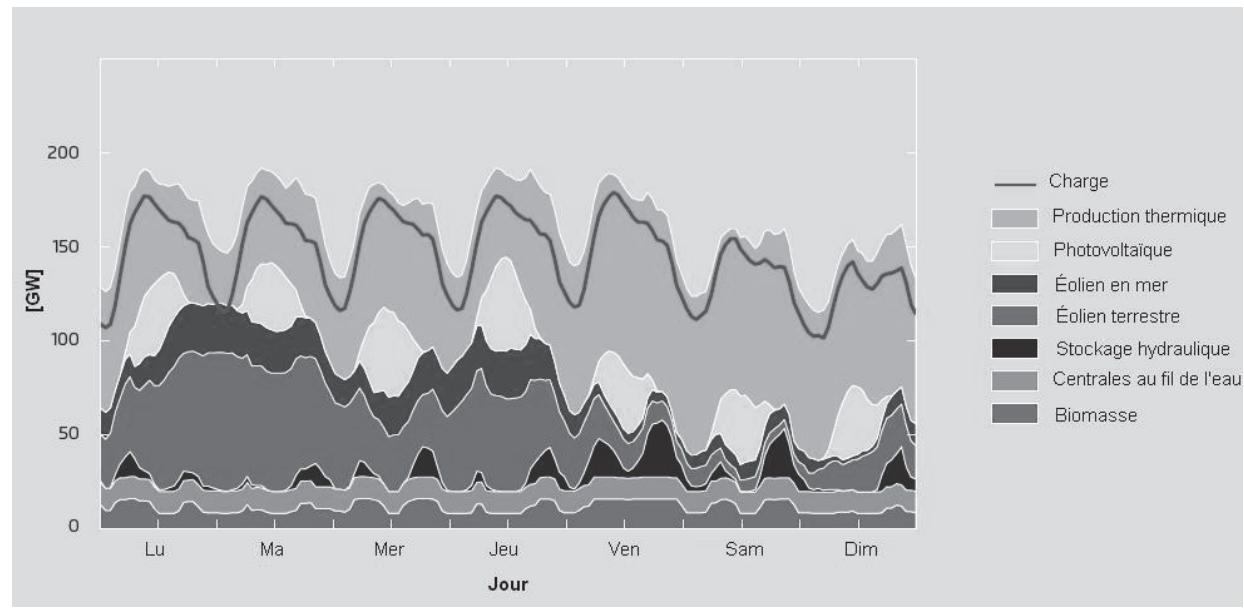

Source : Fraunhofer IWES (2015). Simulation sur la base de l'année météorologique 2011.

En Allemagne, la réponse flexible des moyens de production thermiques est déjà une réalité. Par exemple, lors des jours venteux de décembre 2015, le parc thermique conventionnel a réagi aux variations de prix de manière beaucoup plus flexible qu'on ne le croyait techniquement possible il y a quelques années. Cet ajustement a permis notamment de limiter le niveau des prix négatifs sur le marché. L'éclipse solaire du 20 mars 2015, qui s'est produite lors d'un jour très ensoleillé, représente un autre exemple significatif. Au moment du passage de l'éclipse, la production photovoltaïque a baissé de $12 \mathrm{GW}$ en 65 minutes, puis a augmenté de $19 \mathrm{GW}$ en 75 minutes. Le système électrique allemand est resté parfaitement stable et la flexibilité a été valorisée sur les marchés de court terme. Ces variations étaient extraordinaires en 2015 mais deviendront la norme d'ici 2030, lorsque le système électrique allemand intégrera $50 \%$ d'énergies renouvelables.

Le système électrique français dispose également d'un important potentiel de flexibilité, notamment hydraulique. Ce parc hydroélectrique, composé d'une

\footnotetext{
${ }^{7}$ France, Allemagne, Benelux, Suisse, Autriche.
} 
vingtaine de GW de stockage (court terme, journalier et saisonnier), répond aujourd'hui aux variations de la demande. Dans les années à venir, ce parc hydroélectrique répondra également aux fluctuations des énergies renouvelables. Le parc électronucléaire français est également capable de contribuer techniquement à une partie de ce besoin de flexibilité. Incorporer $40 \%$ de renouvelables implique néanmoins un redimensionnement du parc nucléaire et une modification de son exploitation de court terme vers un régime davantage flexible (voir graphique 5). Les réacteurs les plus récents sont néanmoins capables de fonctionner en suivi de charge et peuvent techniquement contribuer à une partie de ce besoin de flexibilité. Intégrer $40 \%$ d'énergie renouvelable passe néanmoins nécessairement par une réorientation globale de l'exploitation du nucléaire, d'où l'importance des travaux engagés dans le cadre de la programmation pluriannuelle de l'énergie.

Graphique 5 : Corridor de production nucléaire français en 2030, avec $40 \%$ d'énergies renouvelables, et niveau de production en 2013

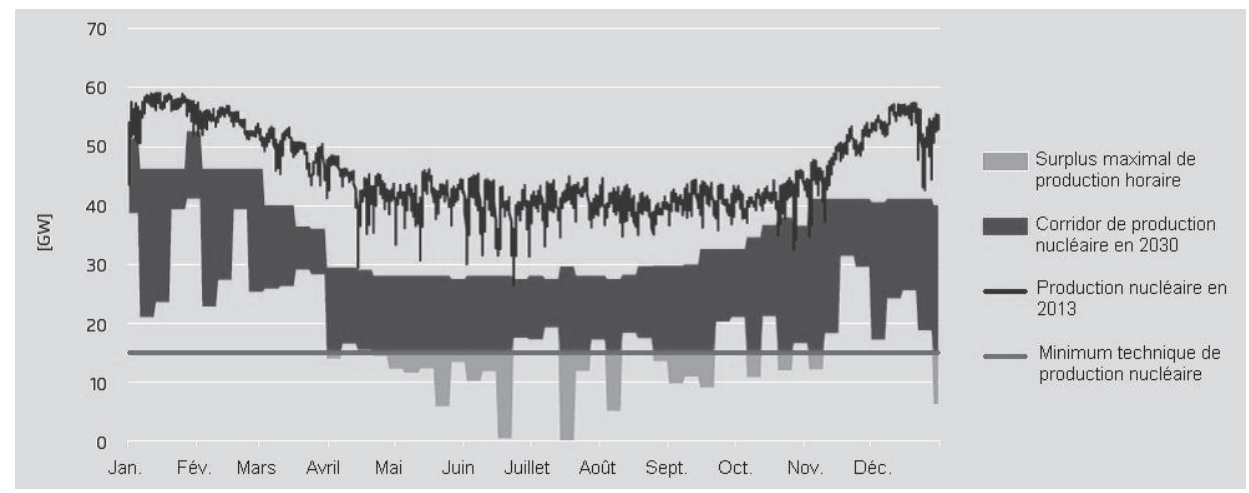

Source : Fraunhofer IWES (2015), sur la base d'Agora Energiewende et RTE.

D'autres options de flexibilité, en particulier la gestion de la demande, le stockage et l'écrêtement ponctuel de la production renouvelable, facilitent l'intégration des énergies renouvelables dans les systèmes électriques. En France, ces options de flexibilité peuvent minimiser le conflit entre intégration des énergies renouvelables et maintien d'un niveau plus élevé de production nucléaire, en particulier lors des semaines caractérisées par de très fortes productions renouvelables. En France, le potentiel de gestion de la demande (le pilotage des chauffe-eau et chauffages électriques, ainsi que la recharge intelligente des véhicules électriques) a été analysé de manière détaillée ${ }^{8}$ et pourrait atteindre près de $25 \mathrm{GW}$ de flexibilité supplémentaire. Le stockage intersaisonnier, en particulier le Power-to-Gas, constitue une option de flexibilité de plus long terme, car aujourd'hui coûteuse, qui interviendra unique-

\footnotetext{
${ }^{8}$ ADEME (2015).
} 
ment à des niveaux de pénétration d'énergie renouvelable très élevés (au-delà de $70 \%)$.

\section{Une optimisation de l'architecture du marché électrique doit permettre de répondre aux besoins accrus de flexibilité}

La flexibilité doit se refléter dans l'ensemble des modalités qui régissent l'organisation des marchés de l'énergie. Les marchés de court terme de l'électricité (journalier, infrajournalier et d'équilibrage) jouent un rôle central dans ce contexte, car ils reflètent au mieux la valeur de l'électricité sur le court terme. Ces marchés mettent en concurrence les diverses options de flexibilité et optimisent les décisions d'une multitude d'acteurs décentralisés sur la base de signaux prix de référence.

Le mouvement de libéralisation du secteur de l'électricité, amorcé dans le milieu des années 1990, a renforcé progressivement la liquidité et le rôle de référence joué par ces marchés. En Allemagne, près de 263 TWh (soit 40 \% de la production d'électricité) ont été négociés sur le marché journalier en $2015^{9}$. Bien qu'une partie importante des échanges se déroule encore hors marché, le prix de marché est la référence des contrats bilatéraux négociés entre producteurs et consommateurs. En France, la fourniture sur le marché de gros a progressé, avec la disparition des tarifs réglementés de l'électricité, effective depuis le $1^{\text {er }}$ janvier 2016, et la baisse des prix de marché qui favorise la concurrence. Les volumes échangés (106 TWh en 2015) y restent inférieurs à ceux observés en Allemagne ${ }^{10}$. Ils sont néanmoins en forte croissance.

L'amélioration de l'architecture des marchés électriques passe par une réduction des barrières à la flexibilité. Un certain nombre d'options «sans regrets » ont été identifiées. Il s'agit notamment d'éliminer les limites de prix (plancher et plafond), afin de refléter au mieux les situations de pénurie ou d'excès de production renouvelable. Il s'agit également de rendre ces marchés plus réactifs, par exemple par l'introduction de produits plus courts. Davantage d'intégration géographique et temporelle joue également un rôle clé, notamment via le couplage des marchés transfrontaliers, en particulier infrajournalier et d'équilibrage.

Ces options «sans regrets » ont été le focus du débat en Allemagne durant l'année 2015. Elles ont également été soulignées dans plusieurs déclarations communautaires et régionales $^{11}$, qui appellent à renforcer le rôle des marchés de l'électricité et la coopération régionale vers davantage de flexibilité.

\footnotetext{
${ }^{9}$ Bundesnetzagentur (2016).

${ }^{10}$ CRE (2016).

${ }^{11}$ Le livre vert et le livre blanc du gouvernement fédéral sur l'architecture du marché électrique, la communication de juillet 2015 de la Commission européenne, la déclaration du Forum pentalatéral et des 12 voisins électriques (juin 2015), ainsi que le mémorandum d'entente (MoU) entre les États de la mer Baltique (juin 2015).
} 


\section{Une réforme du marché des émissions de $\mathrm{CO}_{2}$ et une « mise à la retraite » intelligente des surcapacités thermiques restent nécessaires}

Les marchés électriques européens sont caractérisés par une surcapacité de moyens de production électrique, en particulier des anciennes centrales à charbon fortement émettrices de $\mathrm{CO}_{2}$. Résorber intelligemment cette surcapacité est indispensable pour atteindre les objectifs européens de décarbonation à long terme. Cette priorité concerne en premier plan l'Allemagne, dont le mix électrique est encore largement dominé par le charbon (qui couvre encore $42 \%$ de la production électrique nationale en 2015).

Or aujourd'hui, le marché européen des émissions de $\mathrm{CO}_{2}$ ne délivre pas un signal prix incitatif pour amorcer cette transformation. Une surallocation de quotas de $\mathrm{CO}_{2}$ paralyse le marché et limite totalement le signal d'investissement pourtant nécessaire. Il est donc essentiel de prendre certaines mesures sur le plan communautaire afin de ranimer ce marché, par exemple via l'introduction d'un prix plancher du carbone (par exemple à 30 euros la tonne de $\mathrm{CO}_{2}$ ) et l'élimination des quotas en surplus.

Ces mesures doivent également être complétées le cas échéant par des mesures nationales. L'Allemagne a ainsi décidé de retirer 2,7 GW d'anciennes centrales à lignite entre 2017 et 2020, afin de faciliter l'atteinte de ses objectifs climatiques (réduction des émissions de $\mathrm{CO}_{2}$ de $40 \%$ d'ici 2020 par rapport à 1990). La sortie du charbon doit néanmoins être traitée dans une perspective de long terme, afin d'atteindre les objectifs climatiques du pays, d'accompagner le processus de restructuration socio-économique des régions minières (en particulier la Lusace et le bassin rhénan) et de renforcer la sécurité des investissements dans le domaine de l'énergie. L'adoption d'un plan de sortie du charbon d'ici 2040, développé de manière consensuelle par l'ensemble des parties prenantes, pourrait être une solution en ce sens ${ }^{12}$.

\section{La France est confrontée à d'importantes décisions relatives à l'avenir de son parc électronucléaire}

La France est également confrontée à des choix politiques et économiques majeurs en ce qui concerne le rééquilibrage de son mix électrique et la place qu'y jouera le nucléaire à long terme. Le développement du parc électronucléaire français a nécessité des investissements très concentrés entre 1977 et 1990. De nombreux réacteurs atteindront donc progressivement la fin de leur durée d'exploitation de quarante ans à partir de 2018. Entre 2018 et 2025, ce sont près de $30 \mathrm{GW}$ qui sont concernés (voir graphique 6). Une prolongation de la durée de vie d'exploitation des réacteurs au-delà de quarante ans, qui reste sujette aux avis de l'Autorité de sûreté nucléaire (ASN), nécessite d'importants travaux industriels ( Grand Carénage »). Les coûts de ce programme sont encore relati-

\footnotetext{
${ }^{12}$ Agora Energiewende (2016a).
} 
vement incertains et peu transparents. Selon la Cour des comptes ${ }^{13}$, ils atteindront 100 milliards d'euros entre 2014 et 2030, soit 1,7 milliard d'euros en moyenne par réacteur. Un quart couvre des dépenses d'exploitation (25 milliards d'euros) et les trois autres quarts, des dépenses d'investissement (75 milliards d'euros). Ces coûts sont très sensibles aux coûts du capital, et donc au cadre de financement retenu, ainsi qu'à la durée d'exploitation des installations au-delà de la période de quarante ans. Le coût de la prolongation par kWh dépend ainsi largement de l'autorisation (ou non) d'étendre la durée d'exploitation de deux fois dix ans, comme le souhaite l'opérateur Électricité de France (EDF), et non pas uniquement de dix ans, ce qui est aujourd'hui pourtant l'horizon de décision retenu par l'Autorité de sûreté nucléaire.

Graphique 6 : « La falaise nucléaire » : entre 2018 et 2025, $30 \mathrm{GW}$ de capacités nucléaires en France atteindront 40 ans d'exploitation

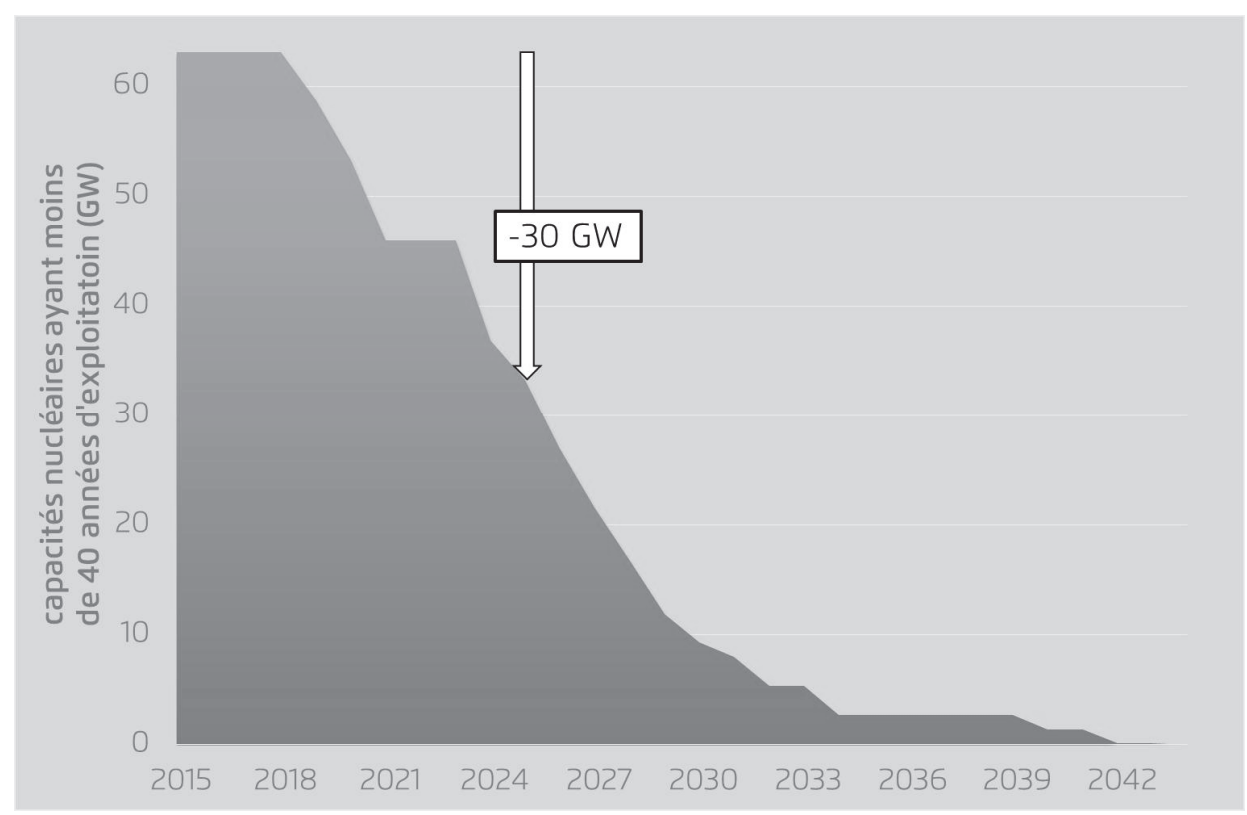

Source : Agora Energiewende, d'après EDF.

Le développement des énergies renouvelables aura vraisemblablement un impact sur le coût de la prolongation du nucléaire en France. Les objectifs retenus par la loi de transition énergétique vont très certainement s'accompagner d'une baisse de la production nucléaire française, dans un contexte de faible croissance de la consommation d'électricité et de capacités limitées à l'export. Cette réduction de la production nucléaire (TWh), à niveau constant de capacités installées $(\mathrm{GW})$, renchérirait mécaniquement le coût de la prolongation du nu-

\footnotetext{
${ }^{13}$ Cour des comptes (2016).
} 
cléaire. À terme, un redimensionnement des capacités nucléaires pourrait donc répondre davantage à une logique économique que politique. Bien que ce rééquilibrage soit implicitement prévu par la loi de transition énergétique (baisse de la part de l'énergie nucléaire de $75 \%$ à $50 \%$ à l'horizon 2025) et son outil programmatique, le pilotage de cette trajectoire en puissance est encore largement incertain. Par ailleurs, dès 2025-2030 commencera à se poser la question du remplacement des centrales nucléaires actuelles par de nouvelles centrales électriques, qu'elles soient nucléaires ou renouvelables. À cet horizon de temps, et sur la base des premiers retours d'expérience des chantiers nucléaires européens en cours, il est fort probable que les énergies renouvelables éolienne et photovoltaïque représentent largement l'alternative la plus économique.

\section{Sécurité d'approvisionnement : les points de vue allemand et français divergent sur la question des mécanismes de capacité}

La question de l'aptitude des marchés de l'énergie (marchés energy only) à garantir seuls les investissements nécessaires à la sécurité d'approvisionnement à long terme fait l'objet d'un débat controversé au sein de l'Union européenne.

Depuis quelques années, on observe une baisse continue des prix de l'électricité sur le marché de gros. En moyenne sur l'année 2015, ils s'établissent à 38,5 euros/MWh en France et 31,6 euros/MWh en Allemagne ${ }^{14}$. Sur le marché à terme pour livraison en 2017, les prix atteignent entre 20 et 25 euros/MWh. Plusieurs facteurs expliquent cette situation : baisse des prix de combustibles sur le marché mondial, stagnation de la demande (conséquence d'une croissance économique faible ainsi que des efforts d'efficacité énergétique) et augmentation de l'offre, à la fois des capacités thermiques conventionnelles et renouvelables. Ces prix bas révèlent une situation surcapacitaire dans pratiquement tous les marchés électriques européens. Ils diminuent les revenus des producteurs, en particulier des producteurs historiques (EDF, E.ON, RWE...). Ce contexte conduit certains observateurs à s'interroger sur la capacité du marché à financer les investissements à venir, lorsque la situation de surcapacité sera résorbée.

Dans certains États membres, en particulier en France, la mise en place d'un marché de capacité ${ }^{15}$ a été proposée, afin de garantir le maintien de suffisamment de capacités dans le système électrique. En France, cette problématique a été discutée dès 2009 dans un contexte structurel et réglementaire très spécifique. Sur le plan structurel, la France est confrontée à une forte thermosensibilité de sa demande électrique, c'est-à-dire que ses pointes de consommation en hiver sont largement supérieures aux niveaux de consommation moyens, une conséquence du recours au chauffage électrique. Sur le plan ré-

\footnotetext{
${ }^{14}$ RTE (2016) ; DNV GL (2015).

${ }^{15}$ C'est-à-dire un marché qui rémunère la capacité disponible (en $\mathrm{MW}$ ) en plus de l'énergie vendue (en MWh).
} 
glementaire, la France considère que la sécurité d'approvisionnement est un bien public, qui est encadré par un critère d'adéquation spécifique ${ }^{16}$ que le système électrique se doit de respecter. Le marché de capacité, qui devrait être effectif dès 2017, a été proposé afin de répondre à ces deux impératifs. À noter néanmoins que les perspectives préoccupantes d'augmentation des pics de consommation faites par le gestionnaire de réseau français depuis les années 2005, et qui ont légitimé l'introduction du mécanisme de capacité, se sont largement résorbées depuis, en particulier grâce aux efforts en matière d'amélioration de l'efficacité énergétique.

Dans d'autres États membres, en particulier en Allemagne, le débat sur les mécanismes de capacité a pris une direction différente. Après une large consultation des parties prenantes, le gouvernement fédéral a refusé l'introduction d'un marché de capacité. Prenant acte de la situation de surcapacité et craignant qu'une rémunération de capacité ne maintienne dans le système des centrales à charbon inflexibles et fortement émettrices de $\mathrm{CO}_{2}$, la priorité a été donnée au perfectionnement du marché de l'énergie. Une réserve de capacité, opérée hors marché, a néanmoins été introduite pour une période transitoire. Cette réserve stratégique, qui peut être actionnée en cas de tension sur l'équilibre offredemande, fait office de «filet de sécurité ». Dans un premier temps, elle rassemblera 2,7 GW d'anciennes centrales à lignite, qui seront maintenues dans le système pour une durée de quatre ans avant d'être déclassées. La réserve stratégique sera ensuite constituée sur la base d'appels d'offres pour une capacité totale de $4,4 \mathrm{GW}$ (environ $5 \%$ de la demande de pointe).

\section{Ces différentes « approches capacitaires » nationales comportent un risque de fragmentation de la politique énergétique européenne}

Compte tenu de l'interconnexion croissante des systèmes électriques européens, la sécurité d'approvisionnement ne peut plus être considérée uniquement sur le plan national. La réalisation de bilans régionaux d'adéquation est une avancée importante qui limite un surdimensionnement des besoins de capacité.

Par ailleurs, les mécanismes de capacité peuvent en principe altérer le bon fonctionnement des marchés de court terme, affaiblissant de facto les efforts entrepris pour améliorer ces marchés. Si un État membre souhaite gérer la sécurité d'approvisionnement par un tel mécanisme, il est essentiel que ce mécanisme soit bien ciblé et qu'il prenne en compte le niveau d'interconnexion et le potentiel de la gestion de la demande, ce que fait d'ailleurs le mécanisme français. La question ne concerne pas uniquement le niveau de capacité requis, mais le type de capacité dont le système a besoin («capabilité »).

Des solutions capacitaires nationales peuvent également entraver a priori la libre concurrence sur le marché européen de l'électricité. Ces mécanismes peuvent avoir des effets redistributifs entre acteurs du marché et entre pays. Par

\footnotetext{
${ }^{16}$ Critère de défaillance LOLE (Loss Of Load Expectation) de $3 \mathrm{~h}$.
} 
exemple, les producteurs d'un pays qui n'ont pas accès à un paiement de capacité sont désavantagés par rapport à ceux qui bénéficient d'une rémunération ${ }^{17}$. C'est dans ce contexte que la France prévoit de faire évoluer son mécanisme, afin de prendre en compte explicitement les participations étrangères. La mise en œuvre de cette disposition, en cours de développement, soulève néanmoins de nombreuses questions techniques et réglementaires.

Ces préoccupations ont conduit la Commission européenne à lancer une enquête sectorielle sur les mécanismes de capacité mis en œuvre dans une dizaine d'États membres, y compris en France et en Allemagne. La Commission a également ouvert une enquête approfondie spécifiquement sur le mécanisme français, afin de s'assurer de sa conformité aux règles de l'Union européenne en matière d'aides d'État. Une harmonisation européenne des mécanismes de capacité nationaux semble aujourd'hui irréaliste, mais un certain niveau de coordination est nécessaire.

\section{Assurer des conditions d'investissement stables en faveur des énergies bas carbone est fondamental pour accompagner leur déploiement}

Les installations d'énergies renouvelables variables sont caractérisées par des coûts d'exploitation (operational expenditure, OPEX) quasi nuls, c'est-à-dire qu'une fois installées, elles produisent quasiment gratuitement lorsque la ressource primaire (soleil ou vent) est disponible. L'investissement initial dans ces installations (capital expenditure, CAPEX) est néanmoins élevé, ce qui nécessite la mise en place de conditions de financement spécifiques. Le nucléaire représente une répartition des coûts similaire (faibles OPEX et CAPEX élevés).

Les conditions de financement des renouvelables sont donc cruciales pour assurer leur compétitivité, et ceci bien que les coûts complets de production de l'éolien et du solaire photovoltaïque soient désormais comparables, voire inférieurs à ceux des centrales thermiques classiques. Un cadre d'investissement favorable aux énergies renouvelables reste donc nécessaire afin de limiter le risque associé au développement des projets et limiter leurs coûts de financement.

Plus fondamentalement, les énergies renouvelables variables sont exposées à un «effet de cannibalisation » lié à leur coût de production marginal quasi nul. Ces technologies produisent de l'électricité lorsque le vent souffle et que le soleil brille, indépendamment du prix de l'électricité sur le marché. Durant les périodes très venteuses ou ensoleillées, les installations produisent une telle quantité d'électricité qu'elles font baisser le prix sur le marché, à partir duquel elles sont censées tirer leurs revenus.

Autres facteurs aggravant la compétitivité des technologies bas carbone : les prix de l'électricité sur les marchés européens sont aujourd'hui extrêmement bas et le prix de la tonne de $\mathrm{CO}_{2}$ est insuffisamment incitatif. Le prix du $\mathrm{CO}_{2}$ va

\footnotetext{
${ }^{17}$ Ces effets redistributifs sont néanmoins contraints par les niveaux d'interconnexions disponibles entre États membres et devraient être relativement limités. Cf. DNV GL (2015).
} 
très vraisemblablement se maintenir à des niveaux insuffisamment élevés dans les années à venir pour inciter au développement des technologies bas carbone. Un prix de 60 euros la tonne de $\mathrm{CO}_{2}$ serait en effet nécessaire pour permettre à ces technologies de récupérer leurs coûts sur le marché.

Il est dans ce contexte très peu vraisemblable que les énergies renouvelables parviennent à se refinancer uniquement sur le marché de l'énergie, en tout cas pas avant 2030, voire 2040. Une approche basée uniquement sur le marché de l'énergie et le marché des émissions de $\mathrm{CO}_{2}$ conduirait à augmenter la prime de risque, et donc le coût des projets. Un cadre de soutien complémentaire reste donc nécessaire, par exemple sous la forme de contrats à long terme, afin de minimiser ce risque, réduire le coût global du déploiement et atteindre les objectifs de développement au rythme et au volume souhaités.

Il est probable qu'un mécanisme similaire soit proposé pour financer les investissements dans le nucléaire, non seulement pour les nouvelles installations (comme c'est d'ailleurs le cas dans le montage financier du projet nucléaire britannique), mais également pour les décisions de prolongation du nucléaire existant. Les prix extrêmement bas observés aujourd'hui sur le marché sont en effet vraisemblablement insuffisants pour financer les investissements importants nécessaires dans le cadre du projet de Grand Carénage.

LA France et L'Allemagne ont engagé des politiques ambitieuses en faveur des énergies renouvelables électriques, en ligne avec les engagements européens à l'horizon 2030. L'éolien et le solaire photovoltaïque sont amenés à jouer un rôle majeur dans cette transition énergétique, conséquence d'une baisse continue de leurs coûts de production. Ces technologies modifient néanmoins profondément les systèmes et marchés électriques européens.

La France et l'Allemagne sont aujourd'hui confrontées à des enjeux politiques de moyen terme différenciés, relatifs à la restructuration de leurs parcs de production conventionnels : nucléaire en France, charbon en Allemagne. Les mix électriques sont néanmoins amenés à converger davantage sur le long terme face au développement significatif des énergies renouvelables.

Préserver des conditions d'investissement fiables sera essentiel pour assurer le déploiement de ces énergies bas carbone au rythme souhaité. Les partisans de l'orthodoxie économique considèrent en général que le marché de l'énergie et le marché européen des émissions de $\mathrm{CO}_{2}$ seront à eux seuls capables de favoriser ces investissements. Une approche davantage pragmatique et holistique est néanmoins nécessaire, afin de prendre en compte la complexité des enjeux économiques et politiques d'un système en transformation.

Dans ce contexte, il apparaît très pertinent de favoriser l'émergence d'une «vision électrique franco-allemande pour l'horizon 2030 », qui bénéficierait à l'ensemble des acteurs économiques et politiques concernés par la transformation des systèmes électriques des deux côtés du Rhin. Cet exercice prospectif commun permettrait d'explorer conjointement les enjeux clés de la transition 
énergétique et les implications de certains choix politiques. Il permettrait également de mieux coordonner les plans nationaux vers l'atteinte des objectifs communautaires.

Renforcer la flexibilité des marchés de l'énergie et restaurer le marché européen des émissions de $\mathrm{CO}_{2}$ sont le préalable à une transformation réussie des systèmes électriques. Ces deux priorités ont déjà été reconnues en France comme en Allemagne. L'avenir de l'architecture du marché électrique devrait intégrer également trois réflexions complémentaires, qui bénéficieraient d'une approche franco-allemande approfondie : comment assurer le maintien de revenus stables pour les technologies bas carbone ? Comment accélérer la « mise à la retraite intelligente » des surcapacités thermiques conventionnelles ? Et comment s'assurer du maintien de la sécurité d'approvisionnement pendant la période de transition ? Au-delà des spécificités électriques nationales de court et moyen terme, ces cinq enjeux seront clés pour permettre le déploiement des énergies renouvelables aux rythmes visés par la France et l'Allemagne à l'horizon 2030.

\section{Indications bibliographiques}

Agence de L'EnVironnement et de LA MAîtriSe de L'Énergie (ADEME), Un mix électrique $100 \%$ renouvelable? Analyses et optimisations, Angers, 2015

Agora Energiewende, Understanding the Energiewende. FAQ on the Ongoing Transition of the German Power System, Berlin, 2015a

Agora ENERgiewende, Coûts d'intégration de l'éolien et du solaire photovoltaïque. Contribution au débat sur les enjeux économiques de l'intégration des énergies éolienne et solaire photovoltaïque dans les systèmes électriques, Berlin, 2015b

Agora Energiewende, Die Energiewende im Stromsektor: Stand der Dinge 2015. Rückblick auf die wesentlichen Entwicklungen sowie Ausblick auf 2016, Berlin, 2016a

Agora Energiewende, Eleven Principles for a Consensus on Coal. Concept for a Stepwise Decarbonisation of the German Power Sector (Short Version), Berlin, 2016b

BUNDESNETZAGENTUR, BUNDESKARTELlaMt, Monitoringbericht 2015, Bonn, 2016

COMmission de RÉGUlation DE L'ÉNERGIE (CRE), Marchés de gros. Observatoire des marchés de l'électricité, du gaz et du $\mathrm{CO}_{2} .4^{e}$ trimestre 2015, Paris, 2016

Consentec, Fraunhofer ISI, R2B, Leitstudie Strommarkt 2015 [étude réalisée pour le compte du ministère fédéral de l'Économie et de l'Énergie], Connect Energy Economics GmbH, Berlin, 2015

COUR DES COMPTES, «La maintenance des centrales nucléaires : une politique remise à niveau, des incertitudes à lever », in COUR DES COMPTES, Le rapport public annuel 2016, Paris, 2016, p. 111-143

DNV GL, Potential Interactions Between Capacity Mechanisms in France and Germany. Descriptive Overview, Cross-Border Impacts and Challenges [étude réalisée pour le compte d'Agora Energiewende], Agora Energiewende, Berlin, 2015

European Network of Transmission System Operators for Electricity (ENTSO-E), Scenario Outlook and Adequacy Forecast 2014-2030, Bruxelles, 2014

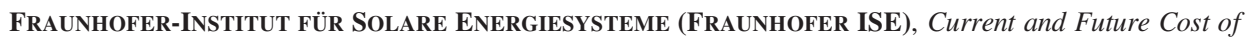
Photovoltaics. Long-term Scenarios for Market Development, System Prices and LCOE of Utility-Scale PV Systems [étude réalisée pour le compte d'Agora Energiewende], Agora Energiewende, Berlin, 2015 
FRAUNHOFER-INSTITUT FÜR WINDENERGIE UND ENERGIESYSTEMTECHNIK (FRAUNHOFER IWES), The European Power System in 2030: Flexibility Challenges and Integration Benefits. An Analysis with a Focus on the Pentalateral Energy Forum Region [analyse réalisée pour le compte d'Agora Energiewende], Agora Energiewende, Berlin, 2015

RÉSEAU DE TRANSPORT D'ÉLECTRICITÉ (RTE), Bilan électrique 2015, La Défense, 2016. 


\section{Le marché européen de l'électricité : un pilier de la sécurité d'approvisionnement ${ }^{1}$}

\section{Wolfram VOGEL}

La sécurité d'approvisionnement est devenue l'une des questions majeures du système électrique en Europe. Les objectifs de I'Union européenne (UE), dans le cadre de sa politique de lutte contre le changement climatique afin de couvrir $27 \%$ de la consommation électrique par la production renouvelable d'ici 2020, ont complexifié la tâche. Grâce à l'arrivée massive des énergies renouvelables, il est question d'une surcapacité en Europe de l'ordre de $100 \mathrm{GW}$, soit l'équivalent de la capacité de production installée en France (cf. Agora Energiewende, 2016).

\section{Le négoce de l'électricité, un enfant de la libéralisation des marchés}

Parallèlement au développement des énergies renouvelables, le secteur énergétique a connu ces deux dernières décennies une transformation fondamentale. Au milieu des années 1990, l'Europe a en effet donné le feu vert à la libéralisation du marché de l'électricité, entraînant le dégroupage de la chaîne énergétique.

Au-delà de la séparation entre la production d'électricité, le réseau de transport et de distribution ainsi que la commercialisation de l'électricité, il s'agissait pour l'UE de créer un marché intérieur européen de l'électricité fortement compétitif.

Cette réorganisation a fait naître de nouveaux besoins, et notamment celui d'organiser le négoce de l'électricité entre les différents maillons de la chaîne de valeur. De cette nécessité sont nées les bourses d'électricité qui assurent un accès non discriminatoire à un marché de gros transparent, fiable et efficace. On parle de « marché organisé de l'électricité » (cf. Conil-Lacoste, 2012).

Le négoce de l'électricité avec des produits standardisés, tel qu'il se déroule tous les jours sur la bourse EPEX SPOT, est donc l'un des résultats les plus visibles de la libéralisation du marché de l'électricité. En ce sens, la bourse de l'électricité s'est à présent établie comme un pilier central de la chaîne de valeur énergétique.

L'électricité présente des caractéristiques physiques qui déterminent sa commercialisation : l'offre et la demande doivent être équilibrées à tout moment de la journée pour garantir la stabilité du réseau ; l'électricité est un produit difficile à stocker ; enfin, la demande est en règle générale peu flexible, tandis que l'offre provenant de sources d'énergie renouvelables devient de plus en plus variable. Le marché de l'électricité reflète ces réalités et s'organise en différents sous-marchés qui se différencient en premier lieu par leurs délais de livraison.

\footnotetext{
${ }^{1}$ Article rédigé en janvier 2017, NdE.
} 


\section{Graphique 1 : L'organisation du marché de l'électricité}

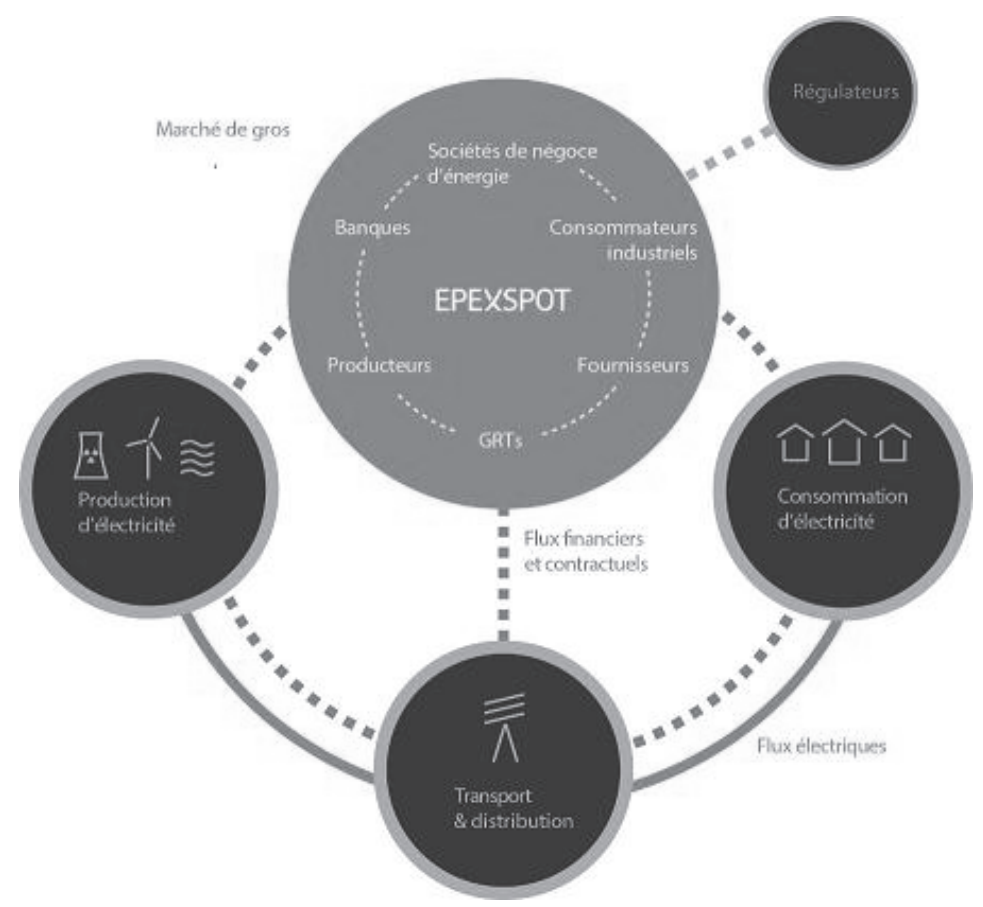

Source : EPEX SPOT.

Sur le marché à terme se négocient des contrats à long et à moyen terme, soit quelques semaines, mois ou années avant échéance. Ce marché sert en première ligne aux acteurs pour gérer le risque prix (couverture/hedging) mais il sert aussi à assurer l'approvisionnement de la demande prévisible (sourcing) ou bien à spéculer. Les quantités fournies sur le marché à terme proviennent en règle générale de centrales électriques conventionnelles, dont la production est planifiable à long terme. Les acheteurs se protègent ainsi des fluctuations de prix à court terme. Ces contrats à terme se soldent soit par une livraison physique, soit par un règlement financier.

Sur les marchés spot, en revanche, ce sont des contrats à court terme qui sont négociés et qui donnent lieu à une livraison physique. EPEX SPOT rassemble producteurs, distributeurs, consommateurs industriels, banques et gestionnaires de réseau de transport qui cherchent à vendre leur production d'électricité ou couvrir leur besoin en électricité sur le court terme. Ces acteurs participent à deux types de marché : le marché J-1 (day-ahead), qui détermine le prix pour chaque heure du lendemain dans le cadre d'une enchère journalière, et le marché infra-journalier (intraday), qui permet aux acteurs de négocier des contrats en continu 24 heures sur 24 , et ce jusqu'à 45 minutes avant la livraison. Les marchés spot contribuent ainsi à l'équilibre à court terme entre la produc- 
tion et la consommation, et se soldent toujours par une livraison physique, c'està-dire une livraison réelle via les réseaux de transport. Le volume d'électricité négocié sur les marchés organisés par la bourse spot de l'électricité s'élève à plus de 500 TWh par an.

\section{Carte 1 : Marchés couverts par EPEX SPOT}

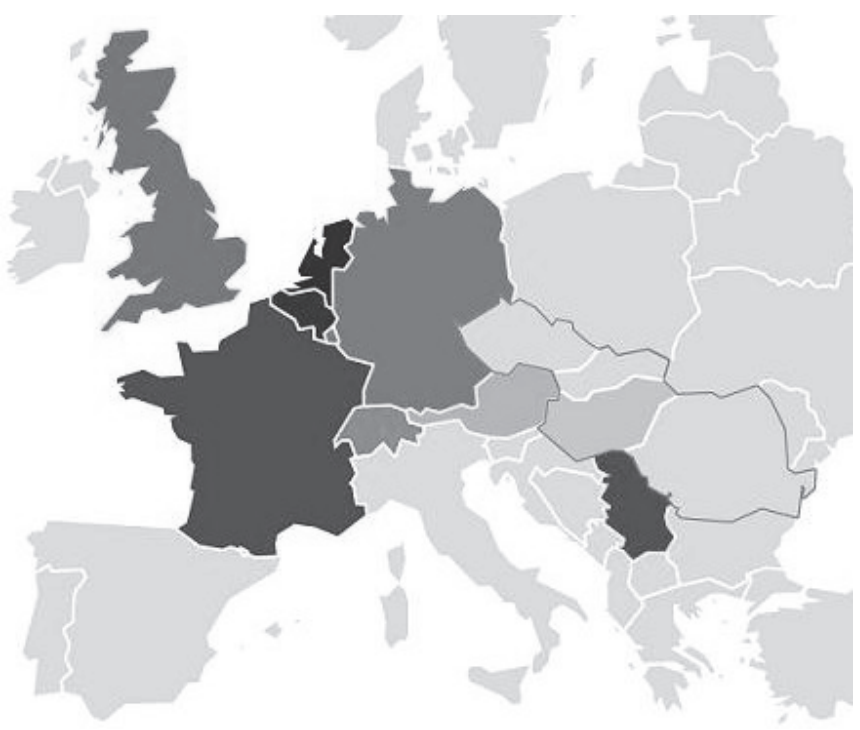

\section{Marchés d'EPEX SPOT}

Allemagne/Autriche/Luxembourg

- France

Grande Bretagne

- Pays-8as

- Belgique

- Suisse

\section{Servir d'autres bourses}

Service doopérations de marché pour la bourse hongroise de l'électricité HUPX

Services de couplage pour la bourse slovaque OKTE, la bourse roumaine OPCOM et HUPX

- Service dopérations de marché pour la bourse serbe de lélectricite SEEPEX (dont $25 \%$ des actions sont détenues par EPEX SPOT)

Source : EPEX SPOT.

En raison des caractéristiques physiques de l'électricité et de l'injection de plus en plus variable provenant de sources d'énergie renouvelables, le négoce à court terme se voit attribuer un rôle toujours croissant. Le marché spot est la plateforme où se négocient notamment les énergies renouvelables, variables et plus difficilement prévisibles sur le long terme.

Les marchés d'ajustement organisés par les gestionnaires de réseau de transport jouent par ailleurs un rôle important dans la fiabilité du système et contribuent à assurer la stabilité du réseau en matière de fréquence et de tension. L'ajustement permet de corriger rapidement tout écart imprévu de production ou de consommation. 


\section{L'évolution du parc de production fait évoluer le prix - et vice versa}

La fonction principale de la bourse de l'électricité consiste à confronter offre et demande de la manière la plus large possible afin de calculer et publier quotidiennement un prix de référence. Celui-ci se détermine par l'intersection entre les courbes d'offre et de demande et correspond en règle générale aux coûts variables de la dernière unité de production dans le «merit order».

Ce prix de référence publié par la bourse est déterminant pour assurer l'efficacité du marché de l'électricité. Il guide les décisions de production et de consommation à court terme, ainsi que les investissements à long terme dans de nouvelles capacités de production. Par ailleurs, il peut aider les consommateurs industriels et résidentiels à moduler/gérer leur consommation. Mais il leur permet aussi de prendre conscience de la réelle valeur de l'électricité et de les sensibiliser à l'éco-citoyenneté. Finalement, ce prix de référence contribue grandement à une utilisation efficace de l'infrastructure du réseau, à son bon fonctionnement et à la sécurité d'approvisionnement.

Tableau 1 : Prix de base (moyenne annuelle) sur les marchés de l'électricité en euros/MWh

\begin{tabular}{|l|c|c|c|c|c|c|c|c|}
\hline \multicolumn{1}{|c|}{ Marché } & $\mathbf{2 0 0 9}$ & $\mathbf{2 0 1 0}$ & $\mathbf{2 0 1 1}$ & $\mathbf{2 0 1 2}$ & $\mathbf{2 0 1 3}$ & $\mathbf{2 0 1 4}$ & $\mathbf{2 0 1 5}$ & $\mathbf{2 0 1 6}$ \\
\hline $\begin{array}{l}\text { DE/AT/LU } \\
\text { (Phelix) }\end{array}$ & 38,85 & 44,49 & 51,12 & 42,60 & 37,78 & 32,76 & 31,63 & 28,98 \\
\hline FR & 43,01 & 47,50 & 48,89 & 46,94 & 43,24 & 34,63 & 38,48 & 36,75 \\
\hline CH (Swissix) & 47,92 & 51,02 & 56,18 & 49,52 & 44,73 & 36,79 & 40,30 & 37,88 \\
\hline
\end{tabular}

Source : EPEX SPOT.

Le niveau actuel des prix ne permet pas à toutes les centrales thermiques d'être rentables. Il faut alors envisager des moyens spécifiques pour leur assurer une rentabilité minimale. C'est l'objectif des mécanismes dits de capacité et des réserves stratégiques. Ces dispositifs, a priori transitoires, ont été proposés principalement de manière indépendante par différents États membres, parmi lesquels la France et l'Allemagne. Ils ont été étudiés par la Commission européenne qui souhaite, tout en les tolérant, les rendre compatibles et ne pas affaiblir le marché intégré de l'électricité qui reste, à ce jour, son grand œuvre. Le projet français de mécanisme de capacité a été autorisé par la Commission le 8 novembre 2016.

\section{La sécurité d'approvisionnement n'est pas une question nationale, mais européenne}

Ce débat ne se limite pas à la simple confrontation "marché energy only » $v s$ «marché de capacité ». Il en découle une multitude de questions : la sécurité 
d'approvisionnement, le prix de l'électricité en tant que facteur économique, l'intégration des énergies renouvelables aux marchés. De plus, les débats se développent à l'échelle nationale et non européenne :

- en Allemagne, le débat s'articule autour de la consultation sur l'organisation du marché de l'électricité de demain (Strommarktdesign von morgen) lancée par le ministère de l'Économie et de l'Énergie. En passant par un livre vert et un livre blanc, il a débouché sur la loi sur le marché de l'électricité qui est en cours de mise en œuvre (cf. BMWi, 2014 ; BMWi, 2015);

- en France et en Grande-Bretagne, le débat est arrivé à son terme. Il est prévu d'introduire un marché décentralisé en France et un marché centralisé en Grande-Bretagne (cf. Stoczkiewicz, 2015).

Il convient néanmoins de différencier les situations nationales. Dans les pays où le marché de l'énergie est mature et pour ceux présentant une surcapacité comme l'Allemagne, nous considérons que les mesures prises sont suffisantes pour continuer de garantir la sécurité d'approvisionnement. D'autres pays faisant face à de sérieux défis en matière de sécurité d'approvisionnement se sont déjà décidés pour le système de mécanisme de capacité, comme en France ou en Grande-Bretagne. Dans ce cas, nous plaidons pour une configuration qui soit compatible avec les objectifs du marché intérieur de l'électricité : c'est-à-dire une configuration basée sur le marché, décentralisée, rentable, « eurocompatible » et neutre en termes de moyens de production.

Incontestablement, l'Allemagne ne rencontre actuellement aucun problème de capacité, mais un problème de répartition. Une grande partie de l'énergie renouvelable est produite dans le nord par les éoliennes à coût marginal zéro, tandis que les centres de consommation se situent dans l'ouest et dans le sud, ce qui représente le défi majeur à relever en matière de transport d'électricité. Le pays se trouve dans une situation de surcapacité, ce qui se répercute sur le signal prix. Personne ne sait aujourd'hui avec certitude si l'Allemagne connaîtra d'ici 2022 une situation de pénurie d'approvisionnement.

Finalement, la question est de savoir comment les politiques vont contourner une telle incertitude : faut-il continuer de développer un marché basé sur le coût marginal (energy only), qui a fait ses preuves au cours des dernières décennies et dont la capacité d'adaptation a été démontrée ? La réponse à cette question ne peut pas être uniquement nationale, mais doit être traitée dans le cadre du marché intérieur européen. La question serait alors de savoir comment garantir la sécurité d'approvisionnement en Allemagne au niveau européen, c'est-à-dire en incluant les capacités de production des pays voisins, le couplage des marchés ainsi que les interconnexions. In fine, cette question concerne tous les États membres de l'UE. Puisque les marchés électriques 
sont couplés, une demande nationale est couverte par l'offre européenne la moins chère. La sécurité d'approvisionnement a ainsi cessé d'être une question nationale. Quelques mesures devraient être mises en place pour sécuriser l'approvisionnement à travers les mécanismes de marché.

\section{L'intégration complète des énergies renouvelables au marché}

Depuis janvier 2012, les producteurs allemands d'énergie renouvelable peuvent choisir de commercialiser directement leur production sur le marché de l'électricité. Ils peuvent se charger eux-mêmes de la commercialisation ou mandater un prestataire. En effet, ces dernières années, plusieurs agrégateurs se sont établis pour prendre en charge la commercialisation des volumes renouvelables pour leurs clients et offrir des services d'analyse et de gestion de portefeuille, de prévision ou d'entretien. En plus des recettes découlant de la vente, réalisée par exemple sur les marchés J-1, infra-journaliers, à terme ou d'ajustement, ces « commercialisateurs directs » se voient rembourser la différence entre le tarif d'achat spécifique au type de l'installation et le prix moyen mensuel de la bourse, sous forme d'une «prime de marché ». Par ailleurs, les risques et les coûts liés à la vente directe sont compensés par une «prime de gestion».

\section{Graphique 2 : Modèle de " prime de marché » en Allemagne}

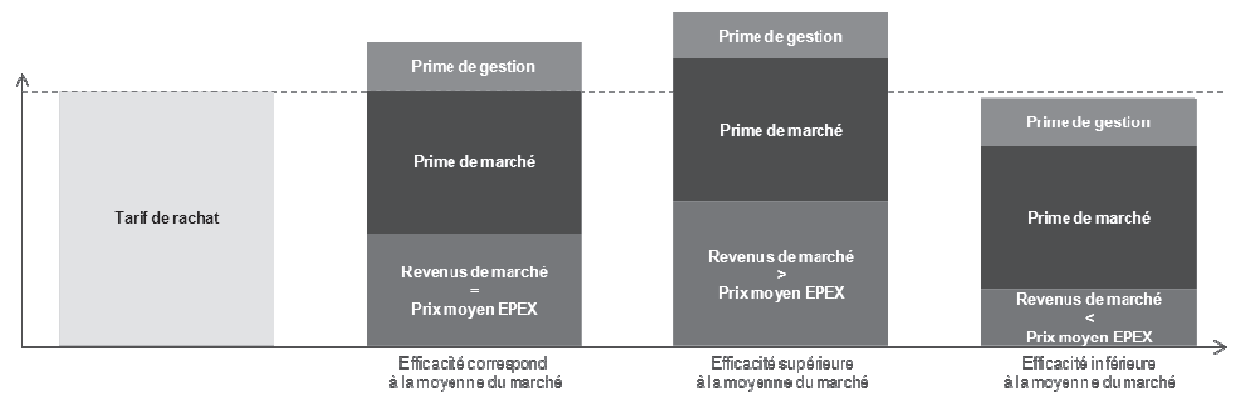

Sources : PwC, gestionnaire de réseau de transport. Analyse : EPEX SPOT.

Tout en renforçant la compétitivité, la vente directe favorise l'intégration progressive des énergies renouvelables dans le marché de l'électricité : les énergies renouvelables agissent sur le marché de l'électricité de manière identique aux autres sources de production, incitant à adopter un comportement vertueux répondant aux besoins du système et à une commercialisation efficace. Ainsi, la précision des prévisions est améliorée et les installations deviennent plus faciles à gérer.

La vente directe connaît une popularité croissante depuis quelques années. Ainsi, fin 2014, plus de la moitié de la puissance renouvelable installée en Al- 
lemagne avait recours au modèle optionnel de la «prime de marché », en particulier dans le secteur éolien. Avec l'entrée en vigueur de la loi allemande de 2014 sur les énergies renouvelables, dite «loi EEG», cette forme de vente directe est obligatoire pour les nouvelles installations dont la puissance dépasse la limite fixée à $500 \mathrm{~kW}$.

\section{Tableau 2 : Puissance installée ayant recours à la vente directe selon le modèle de la " prime de marché "}

\begin{tabular}{|l|r|r|r|r|r|}
\cline { 2 - 6 } \multicolumn{1}{c|}{} & $\begin{array}{c}\text { Déc. 2016 } \\
\text { (en MW) }\end{array}$ & $\begin{array}{c}\text { Déc. 2015 } \\
\text { (en MW) }\end{array}$ & $\begin{array}{c}\text { Déc. 2014 } \\
\text { (en MW) }\end{array}$ & $\begin{array}{c}\text { Déc. 2013 } \\
\text { (en MW) }\end{array}$ & $\begin{array}{c}\text { Déc. 2012 } \\
\text { (en MW) }\end{array}$ \\
\hline Énergie hydraulique & 645 & 631 & 641 & 485 & 392 \\
\hline Gaz & 248 & 249 & 284 & 120 & 42 \\
\hline Biomasse & 5096 & 4760 & 4557 & 2920 & 1936 \\
\hline Géothermie & 30 & 13 & 11 & 5 & 0 \\
\hline Éolien terrestre & 41187 & 36289 & 31868 & 27153 & 23929 \\
\hline Éolien en mer & 4130 & 3269 & 622 & 508 & 248 \\
\hline Solaire & 8235 & 6984 & 5961 & 4297 & 1993 \\
\hline TOTAL & $\mathbf{5 9 5 7 2}$ & $\mathbf{5 2} \mathbf{1 9 5}$ & $\mathbf{4 3 9 4 3}$ & $\mathbf{3 5 4 8 8}$ & $\mathbf{2 8 5 4 1}$ \\
\hline
\end{tabular}

Source : Gestionnaires de réseau de transport. Analyse : EPEX SPOT.

Depuis le lancement du modèle de la «prime de marché », les commercialisateurs directs sont de plus en plus actifs sur la bourse. La fluctuation des prix, qui changent toutes les heures et de plus en plus souvent tous les quarts d'heure, influence considérablement le comportement de ces acteurs. À titre d'exemple, nous pouvons citer les réactions accrues des commercialisateurs directs aux prix négatifs. Depuis l'introduction du modèle de la «prime de marché », on constate le rôle de plus en plus important du signal prix de la bourse lors de périodes de prix négatifs. Les commercialisateurs directs commencent à limiter leurs offres dès l'apparition de prix négatifs. Cette tendance est visible dans les courbes agrégées des prix de l'enchère J-1 d'EPEX SPOT (voir graphique 3). Même si aujourd'hui les commercialisateurs directs ne réagissent pas encore pleinement au signal prix du marché spot, cette tendance représente une évolution importante comparée à l'approche produce-andforget du tarif d'achat fixe.

L'intégration de marché des énergies renouvelables devrait être considérée comme une opportunité pour harmoniser les régimes d'aides européens : les énergies renouvelables devraient avoir un impact grandissant sur les marchés. Il faudrait par ailleurs promouvoir des régimes d'aides transfrontaliers et renforcer les garanties d'origine en tant qu'outil de labellisation de l'électricité. 


\section{Graphique 3 : Courbes agrégées, 9 février 2016 entre 4 et 5 heures}

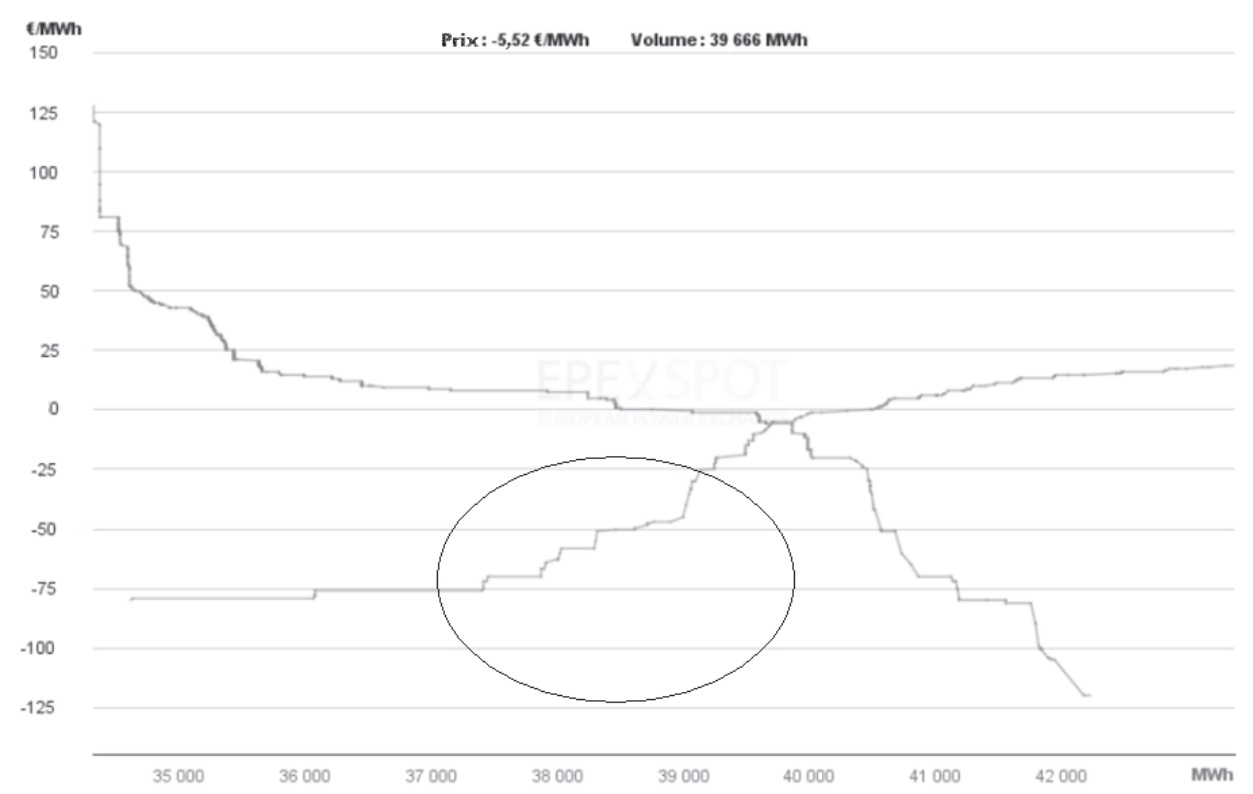

Source : EPEX SPOT.

\section{Valoriser la flexibilité}

Le système de périmètre d'équilibre offre généralement les bonnes incitations économiques permettant de respecter l'équilibre du réseau. La bourse contribue activement, par le biais d'innovations sur le marché, à augmenter la flexibilité du système existant. Afin d'inciter la négociation sur le marché infra-journalier, au lieu de faire appel à l'énergie d'équilibrage, il est correct que le prix de l'énergie d'ajustement prenne en compte le prix infra-journalier.

En 2011, EPEX SPOT a été la première bourse en Europe à introduire les produits quart d'heure sur le marché infra-journalier continu. Les produits quart d'heure permettent en principe de gérer plus efficacement les variations de production et de corriger les écarts de prévision. Les produits quart d'heure font donc sens pour les membres du marché, qui prennent part à la commercialisation directe ou doivent prévoir les fluctuations de production solaire ; mais aussi pour les distributeurs d'électricité, qui peuvent compenser les rampes de leurs centrales conventionnelles et celles de leurs clients au quart d'heure près.

Depuis le lancement des contrats quart d'heure, plus de 21 TWh ont été négociés. Leur part atteint aujourd'hui environ $20 \%$ des volumes infra-journaliers sur le marché allemand. Les incitations à l'équilibrage des zones d'équilibre doivent être augmentées. Dans ce contexte, EPEX SPOT a introduit le 9 décembre 
2014 une enchère quart d'heure sur le marché infra-journalier. L'enchère quart d'heure de l'après-midi (15h) permet la négociation simultanée des 96 quarts d'heure de livraison du jour suivant. Cela permet la concentration de la liquidité et réduit les écarts de prix, au bénéfice du marché.

Cette enchère quotidienne permet aux responsables de périmètre d'équilibre d'adopter une gestion au quart d'heure près des variations de production et une gestion plus fine de leur portefeuille client. Elle permet également une compensation dans l'heure des écarts de prévision. Le signal prix pour les produits quart d'heure permet la plus-value de la flexibilité et encourage dans le même temps les comportements bénéfiques au système. Les volumes négociés sur le marché infra-journalier (ID) se sont développés de manière très dynamique.

Tableau 3 : Volumes infra-journaliers sur le marché allemand (horaires et quart d'heure)

\begin{tabular}{|l|c|c|c|c|c|}
\hline $\begin{array}{c}\text { Volumes } \\
\text { en MWh (DE) }\end{array}$ & $\begin{array}{c}\text { ID Total } \\
\text { (DE) }\end{array}$ & $\begin{array}{c}\text { Quart } \\
\text { d'heure ID } \\
\text { (négociation } \\
\text { continue + } \\
\text { enchère) }\end{array}$ & $\%$ du total & $\begin{array}{c}\text { Enchère } \\
\text { quart } \\
\text { d'heure ID }\end{array}$ & \% du total \\
\hline 2014 & 25853244 & 4936653 & $19 \%$ & 86791 & $0 \%$ \\
\hline 2015 & 36548380 & 7904416 & $22 \%$ & 3956655 & $11 \%$ \\
\hline 2016 & 38808611 & 8273702 & $21 \%$ & 4614405 & $12 \%$ \\
\hline Var. 2015/2014 & $41 \%$ & $60 \%$ & - & - & - \\
\hline Var. 2016/2015 & $6 \%$ & $5 \%$ & - & - & - \\
\hline
\end{tabular}

Source : EPEX SPOT.

\section{Renforcement du signal prix du marché}

Le bon signal envoyé aux acteurs de marché est d'inscrire la libre formation des prix comme modèle cible dans la loi sur le marché de l'électricité. Les prix reflétant la rareté de l'offre, ils représentent une part importante dans la conception du marché. Lorsqu'ils sont utilisés pour valoriser la flexibilité, ils créent des opportunités significatives pour le marché. Un engagement clair contre les interférences réglementaires dans la formation des prix de marché est nécessaire.

Afin de pallier d'éventuelles situations de pénurie, il est nécessaire de permettre sur certaines heures des pics de prix de plusieurs centaines, voire plusieurs milliers d'euros sur le marché. Les pics de prix permettent de couvrir les coûts de maintenance et de production des capacités rarement utilisées. Les pics de prix servent à rentabiliser les installations existantes et à favoriser l'investissement dans de nouvelles centrales. Aussi, de tels pics de prix sporadiques sur le marché de gros n'auraient en moyenne que peu d'impact sur le prix de l'électricité pour le consommateur final, qui ne s'intéresse aujourd'hui que relativement peu au niveau du prix de gros. 
Le prix de référence de la bourse est décisif pour le bon fonctionnement du marché de l'électricité. Les limites de prix techniques sont le fruit d'un consensus européen et donc largement harmonisées. Elles ont fait leurs preuves à leur niveau actuel. Les réflexions portant sur l'harmonisation des limites de prix techniques font l'objet d'une consultation obligatoire dans le cadre du règlement européen sur l'allocation de la capacité et la gestion de la congestion (règlement (UE) 2015/1222).

Seul un marché intérieur de l'électricité européen permet un approvisionnement d'électricité plus sûr et flexible à long terme, car la dimension européenne est la solution en matière d'électricité

L'intégration physique et économique de nos marchés électriques en Europe est une réalité. L'exemple illustrant le mieux que ce marché européen existe est le « couplage des marchés ». Les bourses et les gestionnaires de réseau ont mis en place un système qui permet une utilisation plus efficace de ces interconnexions : pour optimiser les capacités disponibles aux frontières - peu ou mal utilisées avant -, pour faire converger les prix entre les marchés des pays européens respectifs, puis pour améliorer la sécurité d'approvisionnement.

Ce marché intégré a en effet produit, au cours des dix dernières années, des avancées considérables et souvent méconnues, sous la houlette de la Commission européenne. Ainsi, $85 \%$ de la consommation électrique européenne est connectée au travers d'un couplage des marchés conjointement mis en œuvre par les bourses spot de l'électricité et les gestionnaires de réseau de transport. Les capacités de transport aux frontières, calculées quotidiennement par ces derniers, sont introduites dans le carnet d'ordres des bourses spot au moyen d'un algorithme de couplage. Il en ressort que ces capacités de transport sont désormais utilisées à $100 \%$ jusqu'à épuisement et que les électrons vont là où ils sont le plus utiles, de Helsinki à Lisbonne. Il s'agit en quelque sorte d'un « Schengen de l'énergie » dicté par la rationalité économique.

Le couplage des marchés, de par son efficacité, dégage des centaines de millions d'euros de bénéfices par an pour la société européenne. Il a modifié le paradigme de l'indépendance nationale en interdépendance entre États membres, pour améliorer l'indépendance énergétique au niveau de l'Europe. Ce couplage des marchés a coïncidé avec la montée en puissance du renouvelable et a pu en canaliser l'essentiel des effets en élargissant le spectre de la demande au-delà des frontières nationales (cf. Adigbli, 2015).

\section{Une régulation européenne en devenir}

Le troisième paquet énergie de l'Union européenne, entré en vigueur le 3 mars 2011, forme la nouvelle base légale pour les marchés européens de l'énergie. En remplaçant le deuxième paquet de 2003, il a notamment instauré l'Agence de 
coopération des régulateurs de l'énergie (Agency for the Cooperation of Energy Regulators, ACER) et le Réseau européen des gestionnaires de réseau de transport (European Network of Transmission System Operators, ENTSO), pour l'électricité (ENTSO-E) et le gaz (ENTSO-G). L'ACER a été chargée d'établir des lignes directrices permettant à ENTSO-E (secteur de l'électricité) de concevoir des codes de réseau (network codes). Ces codes de réseau définissent de nombreux règlements applicables au réseau électrique, notamment à l'exploitation du réseau, à l'accès aux interconnexions, à la gestion des congestions et à l'énergie d'ajustement. Ils constituent de ce fait le moyen juridique par lequel un marché commun de l'électricité en Europe peut être réalisé. En les intégrant dans le droit de l'UE, les codes de réseau deviennent ainsi juridiquement contraignants (règlements, lignes directrices).

Dans cette optique, le couplage des marchés est devenu victime de son propre succès. Pour achever l'intégration du marché électrique, le couplage est ainsi devenu obligatoire et inscrit dans le règlement européen sur l'allocation de la capacité et la gestion de la congestion. Ce dernier fait partie des codes de réseau mentionnés et vise à promouvoir une concurrence effective dans la production, les marchés de gros et la fourniture d'électricité, à assurer l'utilisation optimale des infrastructures de transport et à respecter la nécessité d'un fonctionnement équitable du marché et d'un processus ordonné de formation des prix (règlement (UE) 2015/1222). Ces règles sont prometteuses et devraient être évaluées au préalable avant d'envisager de nouvelles réglementations.

LE DÉGROUPAGE entre production, transport et commercialisation établit le négoce de l'électricité comme l'un des résultats les plus visibles de la libéralisation du marché de l'électricité. Les acteurs sur le marché de gros ont depuis besoin d'un signal prix qui indique la juste valeur de l'électricité. Puisqu'elle ne se stocke pas, production et consommation doivent être équilibrées en permanence. Les marchés spot contribuent à cet équilibre à court terme en offrant des contrats en J-1 ou en continu 24 heures sur 24 , et ce jusqu'à 30 minutes avant la livraison, se soldant toujours par une livraison physique dans le réseau. La bourse affiche le prix de référence pour chacune des tranches horaires de la journée et contribue ainsi à une utilisation efficace de l'infrastructure du réseau, à son bon fonctionnement et à la sécurité d'approvisionnement. Puisque les marchés européens sont couplés, la sécurité d'approvisionnement a cessé d'être une question nationale. Des mesures ciblées doivent être mises en place pour sécuriser l'approvisionnement à travers les mécanismes de marché : intégrer entièrement les énergies renouvelables dans le marché, valoriser la flexibilité, renforcer le signal prix en admettant des pics de prix sporadiques, évaluer les régulations européennes en vigueur avant de concevoir de nouvelles réglementations. Le marché européen de l'électricité devient ainsi un pilier de la sécurité d'approvisionnement. 


\section{Indications bibliographiques}

Adigbli P., «Voraussetzungen und Folgen einer europäischen Strommarktkopplung », in MüLLER T., KAHL H. (dir.), Erneuerbare Energien in Europa, Nomos, Baden-Baden, 2015, p. 167-184 (Schriften zum Umweltenergierecht ; 21)

Agora Energiewende, The Power Market Pentagon: A Pragmatic Power Market Design for Europe's Energy Transition, Berlin, 2016

BUNDESMINISTERIUM FÜR WIRTSCHAFT UND ENERgIE (BMWi), Ein Strommarkt für die Energiewende. Diskussionspapier des Bundesministeriums für Wirtschaft und Energie (Grünbuch), Berlin, 2014

BUNDESMINISTERIUM FÜR WIRTSCHAFT UND ENERgIE (BMWI), Ein Strommarkt für die Energiewende. Ergebnispapier des Bundesministeriums für Wirtschaft und Energie (Weißbuch), Berlin, 2015

Commission européenne, «Règlement (UE) 2015/1222 de la Commission du 24 juillet 2015 établissant une ligne directrice relative à l'allocation de la capacité et à la gestion de la congestion », Journal officiel de l'Union européenne, $\mathrm{n}^{\circ}$ L197, 25 juillet 2015, p. 24-72

Conil-Lacoste J.-F., «Marchés organisés de l'électricité », in LAPEYRE M. (dir.), Les nouvelles régulations électriques 1. Monopole et concurrence, Lavoisier/Hermes Science, Cachan, 2012, p. 203-244 (EGEM - Génie électrique)

EPEX SPOT, EPEX SPOT. European Power Exchange [en ligne], 2016. Disponible sur : http://www.epex spot.com/en/ [consulté le 08/08/2016]

European Network of Transmission System Operators For Electricity (ENTSO-E), Network Code Overview [en ligne], 2015. Disponible sur: https://www.entsoe.eu/major-projects/network-codedevelopment/Pages/default.aspx [consulté le 08/08/2016]

SToczkiEWicz M., «Capacity Mechanisms in the Electricity Sector in the Context of State Aid », European Energy Journal, vol. 5, n 4 , 2015, p. 29-49. 
$-\mathrm{V}-$

LA COP21

ET LA POLITIQUE EUROPÉENNE DE L'ÉNERGIE 



\section{Protection du climat et transition énergétique ${ }^{1}$}

\section{Berthold GOEKE}

En guise d'introduction, j'aimerais citer un article paru dans l'hebdomadaire Die Zeit du 24 septembre 2015, sous le titre « Ups, wir haben gewonnen"! » (Oups, on a gagné !) Cet article débute par ces mots : "La transition énergétique est le projet modèle de l'Allemagne. On le copie et on le perfectionne dans le monde entier. Est-ce que cet exemple allemand stoppe le changement climatique ? " Le texte parle du journaliste américain Thomas Friedman, qui a visité Berlin en 2015 et, une fois rentré aux États-Unis, a écrit dans le New York Times : "L'Allemagne devient la première puissance verte d'Europe qui fonctionne à l'énergie solaire ${ }^{3}$. " Je trouve que c'est un peu exagéré. Je trouve aussi que cela ne correspond pas au but que nous poursuivons en Allemagne, mais c'est au moins une description de la tendance suivie par le pays avec la transition énergétique entamée ces cinq dernières années. En effet, nous sommes convaincus que la transition énergétique et la protection du climat sont les deux faces d'une même médaille.

\section{La protection du climat est le moteur d'une croissance économique durable}

$\mathrm{Au}$ ministère de l'Environnement comme au gouvernement fédéral, nous sommes tous convaincus que la politique énergétique non seulement peut apporter une contribution décisive à la réussite de la conférence de Paris sur le climat, mais qu'elle doit le faire. En Allemagne et en Europe, la protection du climat est le principal moteur d'une nouvelle politique énergétique durable, et simultanément - je pense important de le souligner - un moteur de croissance économique durable. En effet, nous ne visons pas seulement ceux qui s'inquiètent du réchauffement global de l'atmosphère, mais nous devons aussi impliquer les sceptiques et leur montrer au minimum que nous pouvons aussi apporter des avantages économiques dans la balance. Et c'est ce que nous dit l'équipe de chercheurs de Nicholas Stern : la croissance économique et la protection du climat ne sont pas incompatibles. Au contraire, au XXI ${ }^{\mathrm{e}}$ siècle, la protection du climat est une condition indispensable à une économie moderne et à un bien-être durable.

C'est pourquoi nous saluons l'adoption de la loi relative à la transition énergétique pour la croissance verte en France durant l'été 2015, non seulement parce qu'elle pose les bases du changement dans ce pays ou d'une action commune de

\footnotetext{
${ }^{1}$ Texte issu d'une communication donnée dans le cadre de la journée d'étude La transition énergétique en France et en Allemagne : défis et transformations du marché de l'électricité, coorganisée par le CIRAC le 24 septembre 2015, NdE.

${ }^{2}$ Petra Pinzler, «Ups, wir haben gewonnen » [en ligne], Die Zeit, n 39/2015, 24 septembre 2015, p. [n.c.]. Disponible sur : http://www.zeit.de/2015/39/energiewende-deutschland-vorbild [consulté le 12/08/2016].

3 Thomas Friedman, «Germany, the Green Superpower» [en ligne], The New York Times, 6 mai 2015, p. [n.c.]. Disponible sur : https://www.nytimes.com/2015/05/06/opinion/thomas-friedman-germany-the-greensuperpower.html [consulté le 04/05/2017].
} 
l'Allemagne et de la France dans le contexte européen, mais aussi parce qu'elle constitue un signal encourageant pour la conférence mondiale sur le climat.

Dans l'ensemble, nous pouvons constater que la politique de transition énergétique telle qu'elle a été conçue en France ou qu'elle est actuellement mise en œuvre en Allemagne fait école. D'autres États s'en inspirent. Ils voient à quels problèmes nous sommes confrontés et constatent globalement que nous sommes là sur une voie intéressante. Qui aurait pensé, il y a seulement dix ans, que le photovoltaïque ou l'éolien pourraient produire de l'électricité à un coût compétitif? Aujourd'hui, on sait que cela fonctionne. En Allemagne, les énergies renouvelables couvrent désormais environ un tiers de notre demande en électricité. Celles-ci ont donc supplanté le lignite pour devenir la principale source d'électricité du pays. D'ici 2050, nous voulons renoncer presque totalement aux énergies fossiles. En même temps, nous devons encore améliorer notre efficacité, en matière non seulement de production durable, mais aussi et surtout de consommation d'énergie. Une efficacité énergétique accrue est la clé d'un bien-être durable.

\section{Défis pour la conclusion d'un accord international sur la protection du climat}

Je viens de chercher - notamment avec cet article paru dans Die Zeit - à mettre en évidence une prise de conscience internationale : il nous reste de moins en moins de temps pour procéder à l'indispensable transition vers une économie et un mode de vie respectueux du climat. Même le sommet du G7 qui s'est tenu en juin 2015 au château d'Elmau à Krün, en Allemagne, a donné lieu à un accord entre nos dirigeants : au cours du $\mathrm{XXI}^{\mathrm{e}}$ siècle, l'économie mondiale doit devenir totalement décarbonée, c'est-à-dire s'affranchir complètement du charbon, du pétrole et du gaz naturel. C'est un jalon important pour la politique internationale de lutte contre le changement climatique.

En décembre prochain à Paris, nous devons parvenir à un accord ambitieux sur la protection du climat, en vue d'une économie mondiale neutre pour le climat. Un tel accord nécessite donc :

- une contribution nationale à la protection du climat de la part de tous les États, régulièrement contrôlée et adaptée ;

- un mécanisme d'accroissement des ambitions en vue d'un objectif à long terme ;

- des règles solides et juridiquement contraignantes de transparence et d'imputation, applicables à tous les États.

Actuellement, il ne s'agit pas, comme à Kyoto en 1997, de définir précisément dans le droit international quelles sont les obligations spécifiques à remplir désormais, mais surtout d'enclencher un processus qui fonctionne aussi par un accroissement des ambitions et qui fixe des règles légales de transparence et d'imputation. 


\section{La réduction des émissions de $\mathrm{CO}_{2}$ comme objectif principal}

Pour nous, il est primordial de fixer durablement un prix du carbone valable à l'échelle mondiale et dans tous les secteurs de l'économie. C'est pourquoi nous devons développer et renforcer un marché international du carbone afin de pouvoir atteindre à l'avenir des objectifs ambitieux de réduction des émissions.

En tant que nation industrielle, comment y contribuons-nous ? J'ai déjà mentionné la décision du G7 au château d'Elmau. Au niveau européen, le conseil des ministres de l'Environnement a décidé en septembre 2015 de confier un mandat de négociation exigeant à l'Union européenne (UE), qui nous représente à Paris. L'UE a convenu d'un objectif clair à long terme : atteindre, dans la deuxième moitié de ce siècle, une neutralité climatique durable. Simultanément, les ministres européens de l'Environnement ont décidé de renforcer notablement le système d'échange de quotas d'émission en Europe, en instaurant une réserve de stabilité du marché. Il s'agit là aussi d'une étape décisive vers une réforme ambitieuse du marché européen des quotas d'émission.

En tant qu'Européens, nous prenons actuellement des décisions claires, pour montrer que nous prenons au sérieux la protection du climat, et pour montrer au monde entier que d'autres sont prêts à suivre notre exemple. La protection du climat et l'énergie sont au centre des préoccupations depuis plusieurs années en Europe. Dans le cadre de l'Union de l'énergie, nous nous efforçons de mettre en œuvre les objectifs climatiques fixés par les chefs d'État et de gouvernement de l'UE en octobre 2014. Il s'agit notamment d'atteindre l'objectif européen consistant à réduire de $40 \%$ les émissions de gaz à effet de serre d'ici 2030 (par rapport à 1990), et ce uniquement par des mesures prises au sein de l’Union.

\section{Contribution allemande aux négociations internationales}

Il est clair qu'il nous reste beaucoup de travail à accomplir, y compris pour nous Européens, en particulier pour définir des objectifs contraignants et appropriés en matière de développement des énergies renouvelables, afin de s'assurer que chaque État membre les respecte. En Allemagne, il y a déjà quelques années que nous nous sommes fixé pour objectif, dans le cadre de la transition énergétique, de réduire nos émissions de gaz à effet de serre de $40 \%$ d'ici 2020 . À ce jour, nous constatons que nous sommes certes sur la bonne voie, mais que les mesures prises jusqu'ici ne nous permettront d'atteindre qu'une réduction de $33 \%$ environ.

C'est pourquoi nous avons mis sur pied, en décembre 2014, un programme d'action pour la protection du climat à l'horizon 2020 (Aktionsprogramm Klimaschutz 2020), qui contient plus de 100 mesures supplémentaires afin de combler la différence. En outre, nous préparons actuellement un plan de protection du climat pour la période 2020-2050 (Klimaschutzplan 2050), qui doit montrer aux Allemands comment réussir la décarbonation. Ce programme d'action et ce 
plan climat sont des éléments essentiels de la contribution allemande aux négociations internationales.

\section{POUR CONCLURE, J'AIMERAIS METTRE QUATRE POINTS EN AVANT.}

Premièrement, la contribution décisive pour parvenir à un nouvel accord sur le climat à Paris consiste en une politique d'avenir pour le climat et l'énergie.

Deuxièmement, notre objectif pour Paris est d'obtenir un accord moderne, juste et actuel pour le climat, que tous les États accepteront et qui entrera en vigueur en 2020. Il est particulièrement important de fixer un objectif à long terme, un mécanisme d'accroissement des ambitions et un cadre réglementaire contraignant de transparence et d'imputation.

Troisièmement, Paris doit envoyer un signal clair : le développement mondial doit suivre une voie peu émettrice de carbone et respectueuse du climat. L'objectif visé est une transformation et une décarbonation de l'économie mondiale. Pour cela, tous les États s'engagent à mettre en œuvre des mesures et des réglementations nationales et internationales.

Enfin, quatrièmement - et nous en sommes convaincus -, au XXI $\mathrm{X}^{\mathrm{e}}$ siècle, la protection du climat est indispensable à une économie compétitive et moderne. L'innovation, les investissements et les changements dans le secteur de l'énergie joueront un rôle de premier plan pour assurer une prospérité durable. En tant qu'Allemands, et en tant qu'Européens, nous espérons que les mesures déjà mises en œuvre nous permettront d'être particulièrement convaincants dans les négociations lors de la conférence de l'Organisation des Nations unies (ONU) sur le climat à Paris.

Traduction de Marie-Céline GEORG 


\section{Quelle contribution la politique énergétique peut-elle apporter au succès de la COP21 ?}

\section{Stéphane REICHE}

Ce texte a été rédigé à partir de l'intervention réalisée lors de la journée d'études francoallemande du 24 septembre 2015, organisée par l'Institut allemand de politique étrangère (DGAP), le Centre d'information et de recherche sur l'Allemagne contemporaine (CIRAC) et l'Office franco-allemand pour les énergies renouvelables (OFAEnR)2, au sujet de la transition énergétique en Allemagne et en France. À la suite des sessions sur la compétitivité et la sécurité d'approvisionnement, la troisième session était consacrée au troisième pilier du trilemme énergétique, la protection de l'environnement, avec l'interrogation suivante : "Quelle contribution la politique énergétique peut-elle apporter au succès de la COP21 ? "

\section{Introduction : les conditions de succès de la COP21 - «l'Alliance de Paris pour le Climat »}

En tant que futur président de la COP21, le ministre français des Affaires étrangères Laurent Fabius a en 2015 exposé à de multiples reprises sa vision des conditions de succès de la COP21. Il s'agissait selon lui de construire une «Alliance de Paris pour le Climat », s'appuyant sur quatre piliers :

- un accord universel et juridiquement contraignant ;

- les contributions nationales (ou « $\left.\mathrm{INDC}^{3} »\right)$;

- les moyens de mise en œuvre, financiers et technologiques ;

- la participation des acteurs non-étatiques et de la société civile par le biais de «l'agenda des solutions », également appelé «Plan d'Action Lima-Paris ».

Lors de son discours le 20 janvier 2016 à Strasbourg devant le Parlement européen, Laurent Fabius a estimé que les conditions de succès avaient été remplies par l'accord de Paris ${ }^{4}$. Bien que la somme des contributions nationales à l'effort

\footnotetext{
${ }^{1}$ Article rédigé en mai 2016, NdE.

${ }^{2}$ Devenu depuis l'Office franco-allemand pour la transition énergétique (OFATE), NdE.

${ }^{3}$ INDC: intended nationally determined contributions.

4 «En octobre dernier, j'avais eu l'occasion d'exposer devant vos Commissions de l'Environnement et des Affaires étrangères les critères de ce que serait un «bon accord "à Paris. À travers les 29 articles que comporte l'accord et les 140 points de la décision qui l'accompagne, ces critères ont été remplis. Nous avons obtenu un accord universel, qui repose sur les contributions nationales de 187 pays - c'est considérable. L'accord est reconnu comme ambitieux : il inclut à long terme la limitation du réchauffement climatique à $2{ }^{\circ} \mathrm{C}$, l'engagement à poursuivre les efforts pour atteindre $1,5^{\circ} \mathrm{C}$ et la neutralité carbone dans la deuxième moitié du siècle. Il est dynamique : un mécanisme de revue quinquennale des engagements est prévu, avec un premier rendez-vous fixé en 2023, mais un bilan des efforts pré-2020 aura lieu dès 2018, ce qui permettra une discussion pour un rehaussement des engagements. Concernant le suivi des engagements, un cadre commun de transparence est défini, avec des flexibilités en fonction des capacités de chaque État. L'accord est juridiquement contraignant autant qu'il pouvait l'être compte tenu des contraintes politiques de plusieurs États. Enfin, l'accord est en général tenu pour juste : la notion de différenciation est déclinée sur l'ensemble des su-
} 
de réduction des émissions de gaz à effet de serre ne permette pas encore de limiter le réchauffement climatique à $2{ }^{\circ} \mathrm{C}$ - et encore moins à $1,5{ }^{\circ} \mathrm{C}$ - un dispositif a été mis en place pour une révision à la hausse des engagements nationaux, tous les cinq ans à partir de 2020.

Fort de ce constat, nous allons dans les parties suivantes examiner le rôle joué par l'énergie dans les différentes composantes de cette «Alliance de Paris pour le Climat», que ce soit au niveau des politiques énergétiques nationales (avec les exemples de la France et de l'Allemagne) ou des alliances internationales annoncées lors de la COP21, ou encore de la mobilisation de la société civile au sens large. Enfin, au-delà de ces politiques énergétiques nationales, internationales et locales, nous évoquerons en guise d'ouverture les efforts qui sont à réaliser en dehors du secteur de l'énergie.

\section{Le secteur de l'énergie représente environ deux tiers des émissions de gaz à effet de serre au niveau mondial - quelles politiques nationales pour y remédier?}

Selon un rapport spécial de l'Agence internationale de l'énergie (AIE) de $2015^{5}$, les émissions de $\mathrm{CO}_{2}$ du secteur de l'énergie ${ }^{6}$ représentent environ $60 \%$ des émissions mondiales de gaz à effet de serre.

Plus précisément, toujours selon l'AIE, le secteur de l'énergie représentait en $201068 \%$ des émissions mondiales de gaz à effet de serre d'origine anthropogénique, comme représenté dans le graphique 1 . Les autres principaux secteurs contributeurs sont l'agriculture (11\%) et les process industriels (7\%).

Pour reprendre l'interrogation initiale, la question n'est donc pas seulement de savoir quelle contribution le secteur de l'énergie peut apporter au succès de la COP21, mais plutôt quelle contribution le secteur de l'énergie doit lui apporter. En ce sens, la transition énergétique apparaît bel et bien comme une condition nécessaire à une réduction des émissions au niveau mondial.

Il s'agit alors de comprendre qu'une telle politique énergétique est nécessairement transversale dans son approche. En effet, le secteur de l'énergie recouvre non seulement la production d'électricité et de chaleur ( $42 \%$ des émissions mondiales de $\mathrm{CO}_{2}$ en 2013, toujours selon l' $\mathrm{AIE}^{7}$ ), mais aussi les transports (23\%), l'industrie (19\%), le logement (6\%) et les services (3\%).

Examinons donc comment cette transversalité se décline dans les politiques de transition énergétique française et allemande.

\footnotetext{
jets, et une solidarité des pays riches interviendra envers les pays les plus vulnérables, avec notamment l'engagement de respecter puis de dépasser l'objectif de 100 milliards de dollars annuels au profit de ces derniers et de fixer un nouvel objectif chiffré avant 2025. » Source: http://www.cop21.gouv.fr/wp-content/up loads/2016/01/Discours-LFabius-au-Parlement-europ\%C3\%A9en.pdf [consulté le 04/08/2016].

${ }^{5}$ International Energy Agency (IEA), Key Trends in $\mathrm{CO}_{2}$ Emissions, Excerpt from: $\mathrm{CO}_{2}$ Emissions from Fuel Combustion [en ligne], Paris, édition 2015, p. 3. Disponible sur : http://www.iea.org/publications/freepublica tions/publication/co2-emissions-from-fuel-combustion---2015-edition---excerpt.html [consulté le 04/08/2016].

${ }^{6}$ Le secteur de l'énergie est entendu ici comme la consommation énergétique à laquelle sont ajoutées les émissions fugitives.

${ }^{7}$ Ibid., p. 6.
} 


\section{Graphique 1 : Répartition des émissions mondiales de gaz à effet de serre d'origine anthropogénique (2010)}

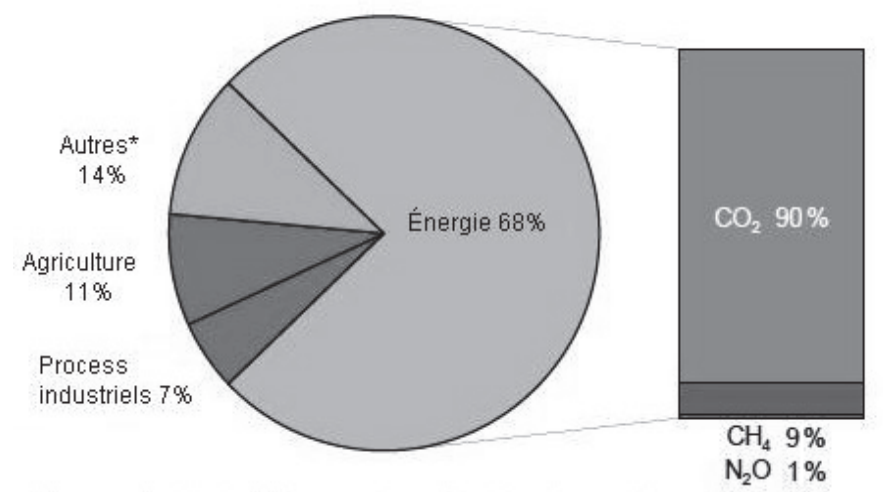

*Autres : combustion de la biomasse à grande échelle, décomposition post-combustion, décomposition de tourbe, émissions indirectes de $\mathrm{N}_{2} \mathrm{O}$ issues d'émissions non agricoles de $\mathrm{NOx}$ et de $\mathrm{NH}_{3}$, déchets, usage de solvants.

Source : International Energy Agency (IEA), Key Trends in $\mathrm{CO}_{2}$ Emissions, Excerpt from: $\mathrm{CO}_{2}$ Emissions from Fuel Combustion [en ligne], Paris, édition 2015, p. 3. Disponible sur: http://www.iea.org/publi cations/freepublications/publication/co2-emissions-from-fuel-combustion---2015-edition---excerpt.html [consulté le 04/08/2016].

\section{La transition énergétique en France}

Au-delà des grands objectifs à moyen et à long terme que sont la réduction des émissions de gaz à effet de serre (-40\% entre 1990 et 2030 ; division par deux entre 1990 et 2050), la réduction de la consommation énergétique finale $(-50 \%$ entre 2012 et 2050) et la réduction de la consommation d'énergies fossiles (-30\% de 2012 à 2030), ainsi que la diversification du mix énergétique (32\% d'énergies renouvelables dans la consommation finale d'énergie en 2030 et $40 \%$ dans la production d'électricité ; diminution de la part du nucléaire à $50 \%$ à l'horizon 2025), la loi de transition énergétique pour la croissance verte adoptée à l'été 2015 présente également des objectifs $^{8}$ en matière de :

- rénovation des bâtiments : 500000 rénovations par an ; $100 \%$ des bâtiments seront au standard «bâtiment basse consommation » en 2050 ;

- développement des transports propres : 7 millions de points de recharge électrique d'ici 2030 ; proportion de $10 \%$ de renouvelables dans la consommation énergétique du secteur du transport en 2020 , et $15 \%$ en 2030 ; obligation d'incorporation de $50 \%$ de véhicules basses émissions lors du renouvellement de flottes de véhicules par l'État et ses agences, et de $25 \%$ pour les collectivités locales ;

- lutte contre le gaspillage et promotion de l'économie circulaire : découplage progressif entre croissance économique et consommation de ma-

\footnotetext{
${ }^{8}$ Source : http://www.developpement-durable.gouv.fr/-Projet-de-loi-.html [consulté le 04/08/2016].
} 
tières premières ; réduction de $10 \%$ des déchets ménagers et assimilés produits d'ici 2020 ; recyclage de $55 \%$ des déchets non dangereux en 2020 et $65 \%$ en 2025 ; valorisation de $70 \%$ des déchets du bâtiment et des travaux publics à l'horizon 2020 ; réduction de $50 \%$ à l'horizon 2025 des quantités de déchets mis en décharge.

Signalons au passage que la réduction des émissions de gaz à effet de serre n'est pas le seul objectif de la loi de transition énergétique, puisqu'elle a également l'ambition de créer de nouveaux emplois (par la « croissance verte»), d'améliorer la qualité de l'air (par le biais notamment des transports propres) et d'augmenter la sécurité énergétique (en valorisant les ressources de nos territoires).

\section{La transition énergétique en Allemagne}

L'Energiewende initiée en 2011 à la suite de l'accident de Fukushima a pris la forme d'une série de briques réglementaires ${ }^{9}$, qui vont de la refonte du soutien aux énergies renouvelables à l'élaboration d'une stratégie d'approvisionnement en gaz, en passant par l'optimisation du réseau électrique - aux niveaux national ${ }^{10}$ et transfrontalier ${ }^{11}$ - ainsi que par une stratégie nationale sur l'efficacité énergétique et une autre sur les bâtiments. Mais du propre aveu des responsables politiques allemands, la transition énergétique en Allemagne s'est pour l'instant surtout caractérisée par le développement des énergies renouvelables dans la production d'électricité. La part des énergies renouvelables dans la consommation électrique est ainsi passée de 20,4 \% en 2011 à 32,6 \% en 2015, tandis que sur la même période elle est, dans la production de chaleur, passée de $11,3 \%$ à (seulement) $13,2 \%$, et dans les transports de $5,6 \%$ à $5,3 \%{ }^{12}$.

La part de l'électricité dans la consommation énergétique finale étant légèrement plus importante en France $\left(22 \%\right.$ en $\left.2014^{13}\right)$ qu'en Allemagne $(21,2 \%$ en $2014^{14}$ ), on constate au total que la part d'énergies renouvelables dans la

\footnotetext{
${ }^{9}$ Cf. la représentation chronologique qu'en a faite le ministère fédéral de l'Économie et de l'Énergie : http:// www.bmwi.de/DE/Themen/Energie/Energiewende/infografik-wichtigste-projekte.html [consulté le 04/08/2016].

${ }^{10}$ Le développement du réseau électrique au niveau national est justifié en Allemagne principalement par la nécessité d'amener l'électricité produite par les éoliennes dans le nord de l'Allemagne vers les bassins de consommation dans le sud de l'Allemagne, mais aussi pour tenir compte d'une production de plus en plus décentralisée.

${ }^{11}$ Une déclaration a été adoptée par l'Allemagne et ses 12 pays « voisins électriques » le 8 juin 2015, afin de favoriser la coopération électrique, cf. http://www.bmwi.de/FR/Presse/communiques-de-presse,did=716332.html [consulté le 04/08/2016].

${ }^{12}$ Source : Umweltbundesamt, http://www.umweltbundesamt.de/themen/klima-energie/erneuerbare-energien/ erneuerbare-energien-in-zahlen [consulté le 04/08/2016].

${ }^{13}$ Source : Richard Lavergne, Pauline Morin, «Chiffres clés énergies-climat en France » [en ligne], in Ministère de l'Écologie, du Développement durable et de l'Énergie, Panorama énergies-climat, édition 2015, Paris, 2015, p. [n.c.]. Disponible sur: http://www.developpement-durable.gouv.fr/IMG/pdf/02_-_Chiffrescles_OK.pdf [consulté le 04/08/2016].

${ }^{14}$ Source : Bundesministerium für Wirtschaft und Energie (BMWi), Arbeitsgemeinschaft Energiebilanzen, Endenergieverbrauch nach Energieträgern [en ligne], juillet 2015. Disponible sur : http://www.bmwi.de/ $\mathrm{DE} /$ Themen/Energie/Energiedaten-und-analysen/Energiedaten/energiegewinnung-energieverbrauch.html [con sulté le 04/08/2016].
} 
consommation énergétique finale est supérieure en France $\left(14,6 \%\right.$ en $\left.2014^{15}\right)$ par rapport à l' Allemagne (entre 13,7 et $13,8 \%$ en $2014^{16}$ ).

Parallèlement au défi de la sortie du charbon dans la production électrique, l'Allemagne se doit de résorber ce retard dans l'émergence des énergies renouvelables dans la chaleur et les transports; c'est pourquoi les appels à un «couplage » des secteurs électricité-chaleur-transports se multiplient. Ce couplage était l'une des priorités majeures mises en avant par le ministre fédéral de l'Énergie Sigmar Gabriel et son secrétaire d'État Rainer Baake les 17 et 18 mars 2016 lors de la deuxième édition du «Berlin Energy Transition Dialogue », qui a vocation à se positionner comme une plate-forme annuelle d'échange mondial de haut niveau sur la transition énergétique.

Avant de généraliser à un cadre international plus large, l'intérêt d'un échange de bonnes pratiques peut s'examiner dans un premier temps à l'exemple du couple franco-allemand.

\section{La coopération franco-allemande pour la transition énergétique}

La France et l'Allemagne héritent d'un mix énergétique sensiblement différent, quand on considère par exemple la part du nucléaire. Pour autant, les deux pays font face aux mêmes défis, en ce qui concerne par exemple l'intégration des énergies renouvelables ou la montée en puissance de l'efficacité énergétique. Il est donc heureux de constater que des structures de coopération bilatérale ont été mises en place, et même se renforcent. Ainsi, un groupe de haut niveau sur l'énergie a été décidé lors du Conseil des ministres franco-allemand du 19 février 2014, qui a ensuite concentré ses efforts sur deux axes de travail : l'incorporation des énergies renouvelables et la sécurité d'approvisionnement électrique.

De façon complémentaire, la plate-forme constituée en 2014 par les deux agences nationales de l'énergie $\left(\mathrm{ADEME}^{17}\right.$ et dena $\left.{ }^{18}\right)$, après avoir défini en 2015 un programme de travail commun, va véritablement monter en puissance au cours de l'année 2016. Une conférence sur la mise en œuvre de la COP21 dans le secteur de l'énergie se tiendra le 14 juin 2016 à l'ambassade de France en Allemagne, associant non seulement l'ADEME et la dena, mais aussi Business France, dans une approche centrée sur les entreprises et leurs solutions technologiques.

De même, l'Office franco-allemand pour les énergies renouvelables verra d'ici cet été son périmètre d'activités étendu et sera à l'occasion de son dixième

\footnotetext{
${ }^{15}$ Source : Richard Lavergne, Pauline Morin, « Chiffres clés énergies-climat en France » [en ligne], in Ministère de l'Écologie, du Développement durable et de l'Énergie, Panorama énergies-climat, édition 2015, Paris, 2015, p. 2. Disponible sur : http://www.developpement-durable.gouv.fr/IMG/pdf/02_-_Chiffres-cles_OK.pdf [consulté le 04/08/2016].

${ }^{16}$ Source : Bundesministerium für Wirtschaft und Energie (BMWi), Zeitreihen zur Entwicklung der erneuerbaren Energien in Deutschland [en ligne], Berlin, février 2016, p. 6. Disponible sur : http://www.erneuerbareenergien.de/EE/Redaktion/DE/Downloads/zeitreihen-zur-entwicklung-der-erneuerbaren-energien-in-deutsch land-1990-2015.pdf?_blob=publicationFile \&v=6 [consulté le 08/08/2016].

${ }_{17}$ ADEME : Agence de l'environnement et de la maîtrise de l'énergie.

${ }^{18}$ dena : Deutsche Energie-Agentur.
} 
anniversaire rebaptisé Office franco-allemand pour la transition énergétique, comme annoncé dans le relevé de décisions du Conseil des ministres francoallemand du 7 avril 2016.

Pour assurer une bonne coordination des positions au sein de l'Union européenne (UE), celle-ci ne doit bien évidemment pas se cantonner à la seule dimension franco-allemande. En particulier, sur les sujets climatiques, une consultation avec les pays d'Europe de l'Est a pu se faire par le biais du triangle de Weimar (Allemagne, France, Pologne), mais aussi de façon élargie avec le groupe de Visegrad, ou V4 (Hongrie, Pologne, République tchèque, Slovaquie). Ainsi, fin septembre 2014, l'Allemagne et la France ont participé à une réunion des pays du V4 associant également la Roumanie et la Bulgarie sur les objectifs énergie-climat de l'UE à l'horizon 2030, trois semaines en amont du Conseil européen du 23 octobre 2014 qui a finalement permis d'adopter de tels objectifs, en particulier celui de réduire d'au moins $40 \%$ les émissions de gaz à effet de serre par rapport à 1990. Ce sont ces objectifs 2030 qui ont constitué la base de la contribution nationale (INDC) de l'UE à la Convention-cadre des Nations unies sur les changements climatiques (CCNUCC). À présent, afin de pouvoir ratifier l'accord de Paris au niveau de l'UE, les États membres doivent se mettre d'accord sur le «partage de l'effort » nécessaire pour réaliser cette réduction de $40 \%$ des émissions.

\section{Zoom sur l'électromobilité - une priorité en France et en Allemagne}

En Allemagne comme en France, la transition énergétique dans les transports passe par l'électromobilité.

L'Allemagne s'est ainsi engagée à ce qu'il y ait un million d'Elektroautos ${ }^{19}$ sur la route d'ici 2020, mais le résultat reste pour l'instant très en deçà de l'objectif : il y aurait actuellement 55000 Elektroautos en circulation. II y a en réalité un problème de « poule et d'œuf » : le nombre de véhicules électriques en circulation étant encore relativement faible, le modèle économique rentabilisant l'investissement dans les infrastructures de recharge n'est pas garanti. Réciproquement, l'insuffisance des infrastructures de recharge peut faire hésiter les particuliers à acquérir de tels véhicules.

En France, le système du bonus-malus et de la prime à la conversion ainsi que le soutien au développement d'une infrastructure de recharge ont contribué à favoriser l'émergence du véhicule électrique au point que la France est devenu le premier pays en volume de ventes de véhicules électriques en Europe, même si la part de marché des véhicules électriques (environ $1 \%$ en France) est encore bien inférieure à celle de la Norvège (environ $15 \%$ ).

En Allemagne, le pas de la prime à l'achat est sur le point d'être franchi, un accord interministériel ayant été obtenu le 26 avril 2016 lors d'une réunion autour de la chancelière fédérale et associant les ministres concernés ainsi que les constructeurs automobiles Volkswagen, Daimler et BMW. Sont prévus 4000 euros pour l'achat d'un véhicule neuf $100 \%$ électrique et 3000 euros pour un véhicule hybride rechargeable.

Des dispositifs de soutien au déploiement des infrastructures de recharge ont également été mis en place dans les deux pays :

- en Allemagne, l'État fédéral consacrera 300 millions d'euros de 2017 à 2020 à l'amélioration des infrastructures de recharge ;

- en France, où il n'existe pas d'objectifs chiffrés sur le nombre de véhicules électriques à atteindre, l'objectif porte sur les infrastructures de recharge : 50000 points de charge accessibles

\footnotetext{
${ }^{19}$ Les Elektroautos ou E-Autos recouvrent ici non seulement les véhicules $100 \%$ électriques, mais aussi les véhicules hybrides rechargeables ainsi que les véhicules hydrogène.
} 
au public en 2020 et 7 millions de points de charge (publics et privés) en 2030. Les fonds du programme des Investissements d'avenir ont soutenu ce déploiement par le biais d'un appel à manifestations d'intérêt.

Du point de vue de l'entente franco-allemande, il est significatif de noter les annonces politiques sur l'électromobilité, par exemple lors du Conseil des ministres franco-allemand du 31 mars 2015, où a été adoptée une déclaration sur l'intégration économique, citant comme cibles d'intérêt commun une infrastructure de recharge transfrontalière des véhicules électriques, la production de cellules de batteries et la mobilité à l'hydrogène ${ }^{20}$; ou lors du Conseil des ministres francoallemand du 7 avril 2016, où les ministres des transports ont annoncé la mise en place d'un groupe de travail franco-allemand "qui traitera surtout des questions liées au déploiement des infrastructures de recharge en électricité et d'hydrogène ainsi que d'autres mesures de politique de transport permettant de renforcer l'attractivité de l'usage de véhicules à moteur électrique ${ }^{21}$ ". Lors de ce même Conseil des ministres franco-allemand, des réflexions ont également été engagées sur la reconversion du bassin d'emploi de Fessenheim, deux options envisagées étant une usine de batteries électriques ou une usine de véhicules électriques (Tesla $)^{22}$.

En ce qui concerne la protection du climat, l'électromobilité est d'autant meilleure que l'électricité consommée est décarbonée. C'est déjà en grande partie le cas en France en raison de la forte part de nucléaire. De plus, en France comme en Allemagne, les objectifs de développement des énergies renouvelables ont vocation à décarboner la production électrique.

En plus de cet effet vertueux contribuant à l'essor des énergies renouvelables, l'électromobilité peut apporter aussi de la flexibilité dans le système électrique, qui devient d'autant plus nécessaire que la part des énergies renouvelables augmente dans le mix énergétique. En effet, on peut imaginer que les véhicules ne soient pas que des consommateurs d'électricité, mais puissent au besoin devenir producteurs (à la manière d'une pile).

Notons également l'appel à projets mondial pour la voiture électrique à 7000 dollars - la « deux-chevaux électrique » - lancé par Ségolène Royal lors de la COP21, tout comme le soutien de la France à la déclaration de Paris sur la mobilité électrique et le changement climatique ${ }^{23}$ qui a été présentée par la «Zero Emission Vehicles Alliance » et qui réunit les régions et les États qui s'engagent pour la mobilité électrique (avec un objectif de $20 \%$ de véhicules électriques d'ici 2030).

Ceci dit, cette alliance n'en est qu'une parmi de nombreuses autres, comme nous le verrons dans la prochaine section.

\section{Les alliances internationales présentées lors de la COP21}

Le troisième pilier de l'Alliance de Paris pour le Climat - les moyens de mise en œuvre - s'appuie sur les transferts financiers et technologiques. Pour les transferts financiers, c'est la somme de 100 milliards de dollars par an qui était la référence au cours des négociations ayant abouti à l'accord de Paris : les

\footnotetext{
${ }^{20}$ Déclaration commune de Sigmar Gabriel et d'Emmanuel Macron $-17^{\mathrm{e}}$ Conseil des ministres francoallemand, 31 mars 2015 à Berlin, http://www.economie.gouv.fr/declaration-franco-allemande-sur-l-inte gration-economique [consulté le 05/08/2016].

${ }^{21}$ Déclaration commune d'Alain Vidalies et d'Alexander Dobrindt $-18^{\mathrm{e}}$ Conseil des ministres francoallemand, 7 avril 2016 à Metz, http://www.developpement-durable.gouv.fr/IMG/pdf/Declaration_ commune_CMFA_-_07-04-16.pdf [consulté le 05/08/2016].

22 Comme expliqué par Ségolène Royal au Grand Jury RTL/LCI/Figaro le 10 avril 2016, cf. http://lci.tf1.fr/politique/segolene-royal-au-grand-jury-pour-une-mutation-industrielle-8731996.html [consulté le $05 / 08 / 2016]$.

${ }^{23}$ Cf. http://www.zevalliance.org/content/cop21-2050-announcement [consulté le 04/08/2016].
} 
fonds publics qui abonderont le Fonds Vert ne représentent certes qu'à peine plus de $10 \%$ de ce montant, mais ils constituent en réalité un levier pour attirer les financements privés nécessaires pour compléter la mise.

Non sans lien, le transfert technologique sera facilité en s'appuyant en particulier sur les alliances internationales présentées lors de la COP21. Une séquence d'évènements inédite s'est en effet tenue pendant la COP21, en parallèle des négociations proprement dites, sous la forme de 12 focus thématiques du $1^{\mathrm{er}}$ au 8 décembre, avec une Journée de l'action de haut niveau le 5 décembre 2015. Une journée était spécialement consacrée à l'énergie.

Depuis la COP21, la France s'efforce de consolider la mise en œuvre de ces coalitions, par exemple l'initiative «énergies renouvelables en Afrique » (installation de $10 \mathrm{GW}$ d'ici 2020), l'Alliance solaire internationale en partenariat avec l'Inde et l'alliance sur la géothermie en partenariat avec l'Islande.

À la croisée entre l'énergie et les transports, 65 pays se sont engagés à améliorer l'efficacité des véhicules dans des pays en développement. Dans le domaine des bâtiments, plus de 60 organisations et 22 pays ont lancé une alliance mondiale sans précédent pour les bâtiments et la construction.

Le prix du carbone, qui constitue la clé de voûte du processus de décarbonation de l'économie et plus particulièrement du secteur de l'énergie, a également fait l'objet d'une coalition lors de la COP21 : la Carbon Pricing Leadership Coalition $^{24}$. Sous sa présidence du G7 en 2015, l'Allemagne avait par anticipation déjà lancé une plate-forme sur le marché du carbone ${ }^{25}$. L'objectif de cette plate-forme est de soutenir l'effort de la CCNUCC dans la démarche de définition de règles uniformes et stables pour l'utilisation de marchés du carbone, qui permettraient à terme de les relier entre eux.

\section{Zoom sur le prix du carbone}

Le prix du carbone peut prendre plusieurs formes, à la fois publiques (taxe, marché de quotas d'émissions, subventions au $\mathrm{CO}_{2}$ évité, valeur tutélaire du carbone) ou privées (prix interne du carbone $^{26}$, marchés de compensation volontaire, désinvestissement du secteur des énergies fossiles). Dans tous les cas, il a vocation à quantifier les coûts (ou « externalités négatives ») engendrés par les émissions de $\mathrm{CO}_{2}$ et à favoriser la décarbonation de l'économie ${ }^{27}$.

Ainsi, la France a introduit une taxe carbone sur le charbon, le fioul et le gaz naturel en 2014, qui a augmenté de 7 euros la tonne de $\mathrm{CO}_{2}$ en 2014 à 14,5 euros la tonne de $\mathrm{CO}_{2}$ en 2015 et 22 euros la tonne de $\mathrm{CO}_{2}$ en 2016. En application de la loi sur la transition énergétique, cette valeur doit atteindre 56 euros la tonne de $\mathrm{CO}_{2}$ en 2020 et 100 euros la tonne de $\mathrm{CO}_{2}$ en 2030. Les entreprises participant au système européen d'échange de quotas d'émission (SEQE) sont exemptées de cette taxe.

Au niveau européen, la France défend l'idée d'un corridor de prix du $\mathrm{CO}_{2}$ afin que le SEQE puisse donner un signal prix suffisant pour orienter les investissements de façon satisfaisante (cf. graphique 2). II faut savoir qu'aux États-Unis, pour les neuf États participant au marché carbone RGGI (Regional Greenhouse Gas Initiative), et au Québec, des corridors de prix sont mis en place. En Chine, les sept marchés carbone pilotes comportent des mécanismes de contrôle des prix.

\footnotetext{
${ }^{24}$ Cf. http://www.carbonpricingleadership.org/ [consulté le 04/08/2016].

${ }^{25} \mathrm{Cf}$. http://www.bmub.bund.de/en/topics/climate-energy/climate/international-climate-policy/carbon-marketplatform/ [consulté le 04/08/2016].

${ }^{26} \mathrm{BP}$ a été l'un des précurseurs en la matière.

${ }^{27}$ Source : http://www.developpement-durable.gouv.fr/Le-prix-du-carbone.html [consulté le 04/08/2016].
} 


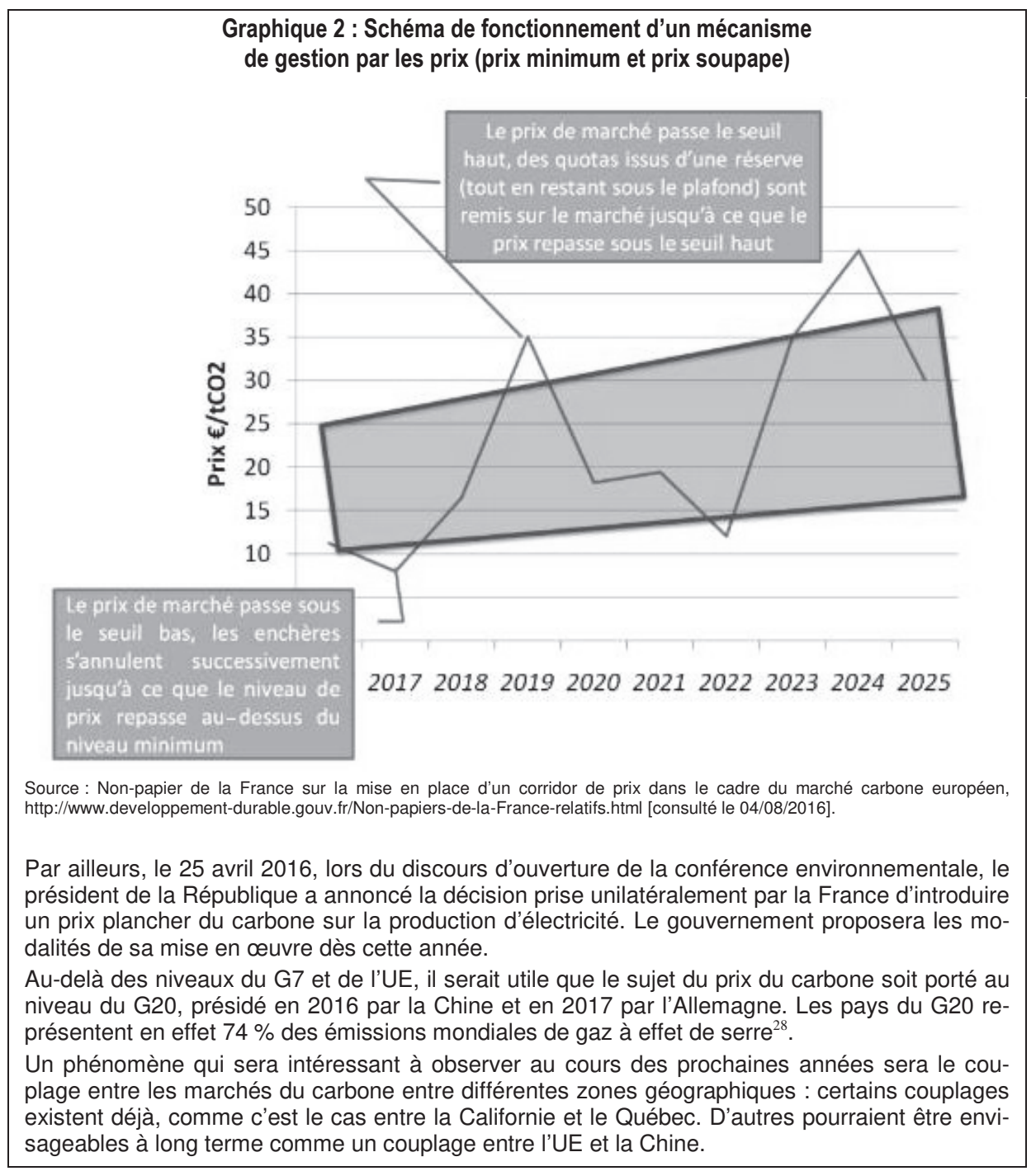

\section{La mobilisation de la société civile}

Par-delà les alliances internationales exposées dans la section précédente et pour revenir sur le quatrième pilier de l'Alliance de Paris, les efforts qui doivent être réalisés dès maintenant reposent d'abord et avant tout sur la mobilisation de la société civile. En effet, ce sont les citoyens en tant que membres de collectivités locales, d'entreprises, de syndicats, d'organisations non gouvernementales (ONG) mais aussi en tant que familles ou simples individus qui ont les clés de

\footnotetext{
${ }^{28}$ Source : http://www.climate-transparency.org/g20-emissions [consulté le 04/08/2016].
} 
la mise en œuvre de la politique énergétique décidée au niveau national. Réciproquement, sans la mobilisation citoyenne, les gouvernements nationaux n'ont pas d'incitation à revoir progressivement à la hausse leur contribution nationale, comme demandé par l'accord de Paris.

Le Plan d'Action Lima-Paris a créé une dynamique qui rassemble plus de 10000 villes, régions, entreprises, investisseurs et associations dans 180 pays.

La plate-forme NAZCA ${ }^{29}$ est une façon de mesurer cette mobilisation de la société civile : 2364 villes, 167 régions, 2090 entreprises et 448 investisseurs s'y sont inscrits. Ainsi, NAZCA centralise et simplifie l'information sur ces engagements climat et permet aux négociateurs de connaître l'ambition et le nombre d'engagements pris par les acteurs de leurs sociétés.

À titre d'exemple, Berlin s'est inscrite ${ }^{30}$ à quatre actions collectives $(C 40$ Cities Clean Bus Declaration, Carbon Neutral Cities Alliance, Compact of Mayors, Covenant of Mayors) et cinq actions individuelles (réduire de $40 \%$ ses émissions de $\mathrm{CO}_{2}$ entre 1990 et 2020 ; couvrir 17,8 \% de la demande énergétique par des énergies renouvelables en 2020 ; réduire de $40 \%$ ses émissions de $\mathrm{CO}_{2}$ entre 2005 et 2020 ; atteindre d'ici 2030 la neutralité carbone pour toute action politique ; réduire de $85 \%$ ses émissions de $\mathrm{CO}_{2}$ entre 1990 et 2050).

Dans les démarches impliquant les collectivités locales, on peut signaler dans la continuité du sommet mondial «climat et territoires » les $1^{\text {er }}$ et 2 juin 2015 à Lyon, le sommet ICCA 2015 les $1^{\text {er }}$ et 2 octobre 2015 à Hanovre ${ }^{31}$, mais aussi l'initiative « Under $2^{\circ} \mathrm{MOU} »$ lancée par le gouverneur de Californie Jerry Brown et le ministre-président du Bade-Wurtemberg Winfried Kretschmann ${ }^{32}$.

Pour revenir sur le franco-allemand, signalons la remise le $1^{\text {er }}$ mars 2016 à Bonn d'une déclaration ${ }^{33}$ de villes françaises et allemandes à la ministre fédérale de l'environnement Barbara Hendricks pour une mise en œuvre de la transition énergétique au niveau local, dans le cadre du programme TANDEM de partenariats franco-allemands pour une transition énergétique au niveau local.

\section{Ouverture : la politique énergétique est une condition nécessaire mais non suffisante pour garantir à l'avenir le succès de la COP21}

Comme nous l'avons vu au début de ce papier, le secteur de l'énergie doit être un contributeur majoritaire à l'effort de réduction des émissions de gaz à effet de serre, mais il ne peut pas réussir à lui tout seul ; il faut aller chercher ailleurs d'autres potentiels d'atténuation.

\footnotetext{
${ }^{29}$ Cf. http://climateaction.unfccc.int/ [consulté le 05/08/2016].

${ }^{30} \mathrm{Cf}$. http://climateaction.unfccc.int/city/berlin/germany [consulté le 08/08/2016].

${ }^{31}$ International Conference on Climate Action, Local Governments Driving Transformation, organisée par le ministère fédéral de l'Environnement, le ministère de l'Environnement du Land de Basse-Saxe et l'Institut allemand d'urbanisme. La déclaration adoptée à l'occasion est disponible sur https://www.icca2015.org/ [consulté le 05/08/2016].

${ }^{32}$ Cf. http://under2mou.org/ [consulté le 05/08/2016].

${ }^{33}$ Cf. http://www.dstgb.de/dstgb/Homepage/Aktuelles/2016/Klimaschutzziele\%20nur\%20mit\%20Kommunen \%20erreichbar/ ou http://bonnsustainabilityportal.de/?p=42125 [consultés le 08/08/2016].
} 
Le programme d'action climat 2020 adopté le 3 décembre 2014 par le gouvernement allemand permet d'ailleurs de quantifier les contributions relatives des différents secteurs de l'économie à un effort de réduction supplémentaire de 62 à 78 millions de tonnes équivalent $\mathrm{CO}_{2}\left(+3\right.$ à $4 \mathrm{Mt} \mathrm{CO}_{2}$ éq. de mesures transversales). Aux côtés de l'efficacité énergétique (25 à $30 \mathrm{Mt} \mathrm{CO}_{2}$ éq.) et de l'évolution du secteur électrique (22 Mt), ce sont les transports (7 à $10 \mathrm{Mt})$, l'industrie et les déchets (3 à 7,7 Mt) et l'agriculture (3,6 Mt) qui y contribuent pour la majeure partie.

Si l'on se réfère à nouveau au bilan du Plan d'Action Lima-Paris ${ }^{34}$, on peut ainsi lister quelques exemples d'initiatives internationales qui ne relèvent pas de la politique énergétique :

- la lutte contre la déforestation : avec un engagement de l'Allemagne, du Royaume-Uni, de la Norvège, de la Colombie, mais aussi de la multinationale Mondelez International ;

- la réduction des émissions dans l'agriculture: l'initiative «4 pour $1000 »$ a comme ambition de favoriser le stockage de carbone dans les sols ;

- les transports : des plans urbains de mobilité durable dans 100 villes à croissance rapide et près de 40 pays grâce à MobilizeYourCity; le développement du fret ferroviaire ;

- les annonces non énergétiques du secteur financier.

De même, sur le portail NAZCA évoqué dans la partie précédente, un certain nombre d'initiatives collectives ou individuelles ne relèvent pas de la politique énergétique stricto sensu.

Dans le domaine des transports, signalons la déclaration du G7 adoptée en septembre dernier sous présidence allemande par les ministres des Transports et par la commissaire européenne en charge des transports, affirmant leur soutien au développement de la conduite autonome et connectée, qui, combinée avec la promotion de systèmes de transport intelligents et durables, y compris la marche et le vélo ainsi que les véhicules propres, peuvent contribuer à un transport routier plus durable. En ce sens, ils soutiendront les efforts à réaliser dans le cadre de l'agenda des solutions, afin de contribuer au succès de la COP $21^{35}$.

Citons pour terminer les politiques de ville durable, qui seront notamment discutées au «German Habitat Forum » début juin à Berlin, en préparation de la conférence «Habitat III» en octobre 2016 à Quito, conférence qui peut être considérée comme la COP21 du milieu urbain.

\footnotetext{
34 Cf. http://www.cop21.gouv.fr/bilan-du-plan-daction-lima-paris-et-de-la-journee-de-laction/ [consulté le 05/08/2016].

35 Cf. http://www.bmvi.de/SharedDocs/EN/Anlagen/VerkehrUndMobilitaet/G7-declaration-on-automatedand-connected-driving.pdf?_blob=publicationFile [consulté le 05/08/2016].
} 
EN CONCLUSION et pour revenir sur la question initiale, "Quelle contribution la politique énergétique peut-elle apporter au succès de la COP21 ? », bien que la communauté internationale se soit félicitée de l'accord trouvé à Paris le 12 décembre 2015 , le succès de la COP21 ne pourra être évalué qu'a posteriori. Le sommet de Paris n'est pas une fin en soi, nous ne sommes qu'au début du voyage.

$\mathrm{Au}$ cours des prochaines rencontres internationales, les évaluations tous les cinq ans à partir de 2018 et les contributions nationales réévaluées à la hausse tous les cinq ans à partir de 2020 sont censées apporter une convergence progressive vers le respect de la limite des $2{ }^{\circ} \mathrm{C}$, voire de $1,5^{\circ} \mathrm{C}$. Nous devrions donc, espérons-le, assister à la mise en place progressive au cours des prochaines années d'une boucle rétroactive entre l'objectif collectif à atteindre et la somme des actions à réaliser ${ }^{36}$.

Comme cela a été évoqué par Hervé Mignon (Réseau de transport d'électricité, RTE) dans son exposé, c'est au niveau des territoires que la transition énergétique et les politiques climatiques de façon générale se joueront in fine, induisant une véritable « révolution des territoires ». Et il est après tout naturel que ce soient les territoires qui portent cette révolution, puisque ce sont eux qui subiront en premier lieu les conséquences du changement climatique.

\footnotetext{
${ }^{36}$ Pour approfondir le lien entre les convictions climatiques du gouverneur Jerry Brown et le « catastrophisme éclairé» du philosophe Jean-Pierre Dupuy, cf. par ex. http://www.sfgate.com/opinion/article/Why-JerryBrown-is-practicing-enlightened-6807422.php [consulté le 08/08/2016].
} 


\section{COP21 : quels enjeux pour l'Europe ?'}

\section{Michel COLOMBIER}

Cette contribution a pour ambition de dresser un bilan de la COP21 et de soulever les questions que les enjeux énergétiques et la diplomatie climatique posent à l'Europe en général, et au couple franco-allemand en particulier, notamment après le vote en faveur $\mathrm{du}$ Brexit de juin 2016.

\section{La COP21 a introduit un changement de paradigme}

De la réduction des émissions...

Dans un premier temps, je souhaiterais replacer la $21^{\mathrm{e}}$ conférence des parties (COP21) de la Convention-cadre des Nations unies sur les changements climatiques (CCNUCC) dans le contexte des négociations de ces 20 à 30 dernières années sur le climat. Pour ma part, j'ai commencé à intervenir dans le processus de négociation peu de temps avant la signature du protocole de Kyoto en 1997 (COP3). Pendant les années qui ont suivi le sommet de Rio en 1992 jusqu'à celui Copenhague en 2009 (COP15), le paradigme qui a prévalu consistait à proposer collectivement de réduire les émissions de gaz à effet de serre en s'appuyant sur les recommandations de la communauté scientifique, dans un contexte de croissance de ces émissions lié notamment à l'émergence de nouvelles économies dans le monde. Cette approche avait conduit à la signature d'une convention à Rio, à une époque où la connaissance scientifique était encore un peu balbutiante et où il s'agissait de stabiliser les émissions mondiales en vertu d'un principe de précaution. La convention de Rio s'est alors construite sur une division du monde classique aux Nations unies, qui consistait à considérer d'un côté les pays industrialisés (dits «Annexe $1 »$ ) et, de l'autre, les pays non industrialisés (dits «non-Annexe $1 »$ ).

\section{... à la décarbonation}

Or cette division du monde correspondait de moins en moins à la réalité, avec l'émergence de la Chine, de l'Inde, de la Corée du Sud ou du Mexique qui n'avaient plus grand-chose à voir avec un certain nombre de pays classés dans la même catégorie. Elle était finalement bien confortable non seulement pour ces pays émergents, qui étaient protégés par le fait qu'on les appelait pays «non-Annexe 1 » et qu'ils n'avaient pas trop d'obligations à remplir, mais aussi parce que, d'un point de vue rhétorique et politique, il était devenu facile pour

\footnotetext{
${ }^{1}$ Texte issu d'une communication donnée dans le cadre du colloque Coopérations franco-allemandes dans la perspective d'une politique européenne de l'énergie, coorganisé par le CIRAC les 26 et 27 septembre 2016, NdE.
} 
les pays du Nord de dire qu'ils ne pouvaient plus s'engager unilatéralement, et ce à la fois pour des raisons d'efficacité environnementale et de compétition économique. Bien que l'adoption du protocole de Kyoto ait été un succès, cette division du monde a ensuite généré des difficultés croissantes dans le processus de négociation.

Dès lors, l'échec de Copenhague a obligé à repenser les choses. Entretemps, plusieurs rapports du Groupe d'experts intergouvernemental sur l'évolution du climat (GIEC) ont été publiés. Il n'y est plus question de seulement réduire les émissions, mais de parvenir à une forme de neutralité carbone au milieu du siècle, ce qui est un tout autre enjeu. En s'appuyant sur cette représentation, les négociateurs, les think tanks ou encore les élus locaux ont progressivement reformulé le paradigme de négociation en amont de la conférence de Paris. L'objectif n'est plus de réduire à moyen terme les émissions de gaz à effet de serre (et donc de savoir qui réduit), mais de parvenir à une décarbonation des économies à la deuxième moitié de ce siècle. Au lieu de chercher une impossible solution diplomatique à des problèmes d'équité qui sont déjà philosophiquement compliqués, mais diplomatiquement totalement insolubles, il s'agit d'avancer collectivement - et individuellement - vers la décarbonation. Or cela nécessite une transition économique et technologique vers un modèle de prospérité différent, qui se déploie dans un nouveau contexte de régulation.

\section{Principe d'universalité}

À partir du moment où un certain nombre de pays adoptent cette approche, cela influe sur tous les pays parce que ne pas s'inscrire dans cette perspective, c'est prendre le risque de rester en dehors de cette transition, ce qui n'est pas forcément une bonne chose.

Entre la conférence de Cancún en 2010 (COP16) et celle de Paris fin 2015, de nouveaux acteurs sont entrés dans la négociation, comme les collectivités locales, mais aussi les milieux financiers, qui ont beaucoup travaillé sur les risques liés au changement climatique, mais aussi sur les risques que des changements de politiques radicaux peuvent faire peser sur les investissements. Ces nouveaux acteurs ont progressivement fait émerger l'idée que tous les pays sont concernés par cette transition. Dans ce contexte, la négociation porte sur la reconnaissance de cette nécessité et sur les questions de solidarité et d'équité, qui perdurent depuis le sommet de Rio, mais qui vont devoir être traitées autrement. Il s'agira en effet de déterminer les coopérations politiques et économiques qui vont permettre à l'ensemble des pays, y compris les pays en développement, de s'engager dans cette transition.

Cette reconnaissance, par l'ensemble des pays, que chacun est concerné et doit s'engager dans la lutte contre le changement climatique est ce qui fait le succès de la COP21, mais aussi sa fragilité : 192 pays ont élaboré une politique prévisionnelle, plus ou moins ambitieuse, de réduction des émissions et d'adaptation, 
ou INDC (intended nationally determined contribution). Cet engagement de tous les pays constitue un formidable progrès. Il y a quelques années encore, certaines grandes économies mondiales ne souhaitaient pas s'impliquer dans ce processus, ce qui créait une impasse sur le plan diplomatique.

L'approche universelle obtenue à Paris a été qualifié de bottom-up. Je pense que c'est une aberration parce que la discussion de Kyoto n'était pas une discussion top-down. Quoi qu'il en soit, chaque pays a exposé son projet pour atteindre l'objectif collectif de décarbonation. Dans des grands pays comme l'Indonésie, l'Australie ou l'Afrique du Sud, ces projets de décarbonation ont fait l'objet d'un vaste débat, relayé dans la presse, entre les ministères des Finances, de l'Industrie et les ONG (ce qui est moins le cas dans de plus petits pays).

Si la mise en œuvre des projets de tous les pays aboutit à l'horizon 2050 à un niveau d'émissions encore trop élevé par rapport à celui qu'il faudrait atteindre selon les scientifiques, il existe néanmoins désormais une référence pour tous les pays. De plus, cette référence constitue une avancée, parce que certains pays y ont tout de même mis une certaine ambition.

\section{L'accord de Paris est avant tout un accord de règles}

Dans le cadre des accords environnementaux qui ont précédé la COP21, l'idée était de négocier des quantités pour une période donnée et de les réviser pour la période suivante. Anticipant les difficultés liées aux projets des différents pays, l'accord de Paris est avant tout un accord de règles, où il n'est pas question de quantités. De plus, certains pays comme les États-Unis n'auraient pas pu le ratifier s'il avait compris des quantités. Enfin, l'accord de Kyoto a montré que prévoir de nouvelles quantités pour une période ultérieure obligeait à ratifier de nouveau un accord, ce qui est compliqué, et risqué, d'un point de vue politique. Désormais, tout l'enjeu sera de savoir si ces règles peuvent fonctionner. Dans ce contexte, l'Europe aura un rôle important à jouer.

Un accord de règles a pour ambition de tenir en permanence une tension de négociation entre les pays vers une augmentation de l'ambition des politiques. Il ne s'agit donc pas de se rencontrer de nouveau en 2028 pour parler des objectifs à l'horizon 2040, mais de discuter d'ici cinq ans à l'échelle mondiale de l'avancée des politiques proposées à l'horizon 2030 dans le cadre de l'accord de Paris, en tenant compte de l'évolution de la science et des retours d'expérience de la mise en œuvre des politiques. On attend de cela à la fois plus d'ambition et une progressive convergence possible, non seulement des objectifs, mais aussi des politiques à mettre en œuvre.

\section{Quatre questions sont posées à l'Europe}

Depuis Rio, puis Kyoto, l'Union européenne (UE) a joué un rôle moteur dans les négociations sur le climat, même si le modèle issu de Kyoto provenait davantage des Américains. Cela est dû notamment à la confiance qui régnait entre 
l'Europe et les pays en développement et à l'ambition affichée du continent pour faire face au défi climatique, en comparaison avec quelques zones économiques et politiques plutôt récalcitrantes au départ.

\section{L'ambition}

L'accord de Paris s'accompagne de quatre enjeux pour l'Europe. Le premier concerne l'ambition. Du fait de la situation actuelle au sein de l'UE, ce ne sera certes pas le sujet du prochain Conseil européen, mais c'est un problème qu'il va falloir résoudre pour deux raisons. Premièrement, l'objectif inscrit dans l'INDC de l'UE, qui prévoit une réduction de $40 \%$ des émissions d'ici à 2030 (par rapport à 1990), n'est pas suffisant pour atteindre l'objectif européen de long terme de -80 à $-95 \%$ d'ici à 2050 . De plus, la volonté de la communauté internationale de parvenir à une neutralité carbone à 2050 oblige l'Europe à revoir son objectif de long terme. Par conséquent, l'Europe n'est plus vraiment le continent de l'ambition.

\section{Le projet industriel et économique}

Puisque l'accord prévoit de rediscuter des objectifs à l'horizon 2020, l'Europe ne peut pas se permettre, d'un point de vue strictement politique, de ne pas revoir ses ambitions à la hausse d'ici là. Toutefois, elle n'est pas en situation d'ouvrir cette discussion actuellement.

En effet, il lui faut d'abord retrouver un projet industriel et économique sur le climat. La vision d'un projet de transition, qui a désormais été inscrite dans l'accord de Paris, est paradoxalement ce qui a porté l'Europe pendant un certain nombre d'années après Kyoto. Je me rappelle qu'entre 2003 et 2005, quand nous préparions le système européen d'échange de quotas d'émission de gaz à effet de serre (SEQE) puis le premier paquet énergie-climat, des délégations issues notamment de Chine, mais aussi d'autres pays, venaient nous interroger sur ce projet européen qui était présenté comme un projet industriel, politique et de régulation économique proposant une prospérité sur une base énergétique différente, portée par l'innovation technologique. Le fait que les Européens croient à cette transition - qui ne remportait pas l'adhésion des Américains - interrogeait beaucoup nos partenaires étrangers. Or l'Europe a progressivement perdu cette vision. Nous nous sommes beaucoup penchés sur les aspects techniques pour améliorer le SEQE ou sur les enjeux de partage de l'effort (burden sharing), mais nous avons perdu l'élan du début des années 2000 sur le projet de transition.

\section{La gouvernance}

Pour mener à bien ce projet, l'Union de l'énergie constitue un cadre, du moins en partie, car le climat va au-delà de l'énergie. Indépendamment de la question 
anglaise, la gouvernance top-down qui a existé jusque-là dans la discussion sur les paquets énergie-climat, avec une répartition des efforts par pays, a bien fonctionné quand il s'agissait d'objectifs moins ambitieux en termes de réduction des émissions ou de développement des énergies renouvelables. Dans le contexte actuel d'une transformation beaucoup plus radicale de nos systèmes énergétiques, il est nécessaire d'avoir une adhésion des milieux économiques et des populations. De plus, la transition se fait aussi en tenant compte de la situation actuelle, et ces projets de transition ne peuvent être identiques d'un pays à l'autre. En Pologne, l'enjeu de la sortie du charbon est encore bien différent de ce qu'il est en Allemagne ou même en France, où il ne reste que quelques centrales à charbon. De plus, il n'est pas sûr que tous les pays souhaitent aller dans la même direction. Par conséquent, l'Europe a besoin d'un projet politique beaucoup plus participatif, qui se construise sur ce que veulent les pays, mais aussi collectif, ce qui implique que la gouvernance soit réévaluée par rapport à ce qui a été fait jusque-là dans le paquet énergie-climat et que la Commission joue rôle différent.

\section{La coopération}

Le dernier point sur lequel je pense qu'il faut que l'Europe soit rapidement très active porte sur les relations extérieures et la coopération. La qualité de la relation de l'Europe au monde en développement a contribué à ce qu'elle joue un rôle de déblocage dans la négociation sur le climat. Celle-ci repose non seulement sur des raisons historiques, mais aussi sur des institutions comme l'Agence allemande de coopération internationale (GIZ), l'Agence française de développement (AFD) et la direction générale de la coopération internationale et du développement de la Commission européenne (DG DEVCO). Après Kyoto, l'Europe a notamment fondé sa coopération sur le mécanisme pour un développement propre (MDP) qui consiste, pour les pays industrialisés, à financer des projets dans des pays moins riches pour y réduire les émissions, en échange de quoi ils reçoivent des crédits pour atteindre leurs propres objectifs de diminution des émissions.

Il est naïf de penser qu'à la conférence de Paris, un accord du même type que celui de Kyoto aurait permis de fixer un prix mondial du carbone. Avoir des engagements quantitatifs ne garantit pas d'avoir un marché du carbone. Quoi qu'il en soit, il est nécessaire d'aborder la question du lien entre les prix du carbone régionaux. Un article dans l'accord de Paris reproduit plus ou moins le MDP.

Actuellement, les pays en développement sont majoritairement méfiants, non pas par rapport à l'utilisation du prix du carbone en tant que politique domestique, mais en tant que base des relations économiques, technologiques et financières entre les pays du Nord et les pays du Sud, comme cela apparaît dans les INDC. Les pays en question vont solliciter des investissements étrangers, mais vont souhaiter s'approprier politiquement les réductions d'émissions. 
Dès lors, il nous faut retrouver un modèle d'engagement avec les pays en développement et de financement de la coopération que nous avons un peu perdu collectivement au niveau européen. Et il nous faut retrouver aussi une certaine confiance de certains pays du Sud qui nous ont trouvés, nous les Européens, un peu dogmatiques sur ce point. Il reste donc un travail à fournir en ce qui concerne les politiques de coopération technologique et les politiques de soutien financier.

L'ACCORd de PARIS CONSTITUe CERTES UNe ÉTAPE IMPORTANTE dans la lutte globale contre le changement climatique, mais il ouvre surtout une période nouvelle à laquelle il propose un cadre de règles qu'il faudra rendre opérationnelles. L'Europe, longtemps leader sur le plan multilatéral mais aussi sur sa dynamique politique interne, a vu son image affaiblie par ses difficultés récentes et la montée en puissance du couple sino-américain. Les revirements à attendre à la suite des élections américaines vont placer la Chine dans une position centrale, mais l'Europe conserve bien évidemment un rôle essentiel pour assurer la poursuite d'un accord qu'elle a très largement contribué à construire.

La dynamique internationale repose désormais sur un jeu complexe entre la CCNUCC et la mise en œuvre domestique, et entre les États parties à la convention et les participants non étatiques. La capacité de l'Europe à retrouver son propre élan interne sur la question climatique sera donc plus que jamais déterminante pour son influence internationale. Avec le Brexit, la France et l'Allemagne sont en première ligne sur ce front, avec des approches de la transition énergétique certes différentes, mais dans les deux cas bien réelles et portées, audelà des États, par leurs sociétés civiles et un nombre croissant de collectivités locales et d'entreprises. Plus que la Commission, c'est ce couple qui peut et doit aujourd'hui reconstruire une vision européenne mobilisatrice. 


\section{La politique énergétique et climatique de l'UE après la COP21}

\section{Christian EgENHOFER}

En tant que responsable du programme Énergie et climat au Centre d'études politiques européennes (CEPS) à Bruxelles, je m'occupe de politique énergétique, de finances, de politique climatique et de marchés. Dans cet exposé, je vais tout d'abord analyser les conséquences de la conférence de l'Organisation des Nations unies (ONU) sur le climat (COP21) pour l'Union européenne (UE) et sa politique climatique. Ensuite, je m'intéresserai à la politique énergétique et climatique actuelle en Europe. Troisièmement, j'aimerais aborder la transition énergétique ou plutôt les transitions énergétiques, les investissements et la compétitivité en tant que principaux chantiers à réaliser. Enfin se pose la question de ce qui se passera en Europe après 2030.

\section{Les conséquences de la COP21 pour l'UE}

Dans le cadre de la conférence de l'ONU sur le climat qui s'est tenue à Paris en 2015, les objectifs suivants de réduction des émissions de gaz à effet de serre ont fait l'objet d'un accord : au moins $40 \%$ d'ici 2030, et 80-95\% d'ici 2050 (par rapport à 1990). Un contrôle aura lieu tous les cinq ans. Le point décisif est toutefois que la conférence de Paris n'a donné aucune instruction précise sur ce que l'UE doit faire. Celle-ci est seule responsable de la mise en œuvre de cette stratégie, via son processus législatif.

Par exemple, en matière d'énergies renouvelables, on propose de fixer des objectifs contraignants à l'échelle de l'UE, mais il n'y a actuellement aucun débat européen pour les atteindre. Il en va de même pour le système européen d'échange de quotas d'émission. Beaucoup de ces questions d'ambition n'en sont qu'au processus de discussion législatif de l'UE. Cela signifie que les choses peuvent durer, avec une issue incertaine.

Le point suivant concerne le fait que le débat sur le climat pour 2020, 2030 et après dépend fortement de la situation politique d'ensemble dans l'UE. Depuis le traité de Lisbonne - et même avant, en principe -, c'est le Conseil européen qui assume la direction, y compris pour la politique climatique et énergétique. À cause des autres grands thèmes de l'UE (l'euro, la crise des migrants, le Brexit) dont il faut débattre en ce moment et qui s'imposeront pendant longtemps encore, il semble difficile de parvenir à organiser un débat de fond pourtant nécessaire sur la politique climatique et énergétique de l'UE.

\footnotetext{
${ }^{1}$ Texte issu d'une communication donnée dans le cadre du colloque Coopérations franco-allemandes dans la perspective d'une politique européenne de l'énergie, coorganisé par le CIRAC les 26 et 27 septembre 2016, NdE.
} 
De plus, une grande partie des États d'Europe du Sud-Est n'ont pas la volonté politique de mettre en œuvre une politique climatique et de renoncer au gaz et au charbon, notamment à cause d'un haut degré de corruption.

\section{La politique énergétique et climatique de l'UE : l'Union de l'énergie}

L'Union de l'énergie est un paquet global qui réunit les questions de politique énergétique et climatique et, dans la plus pure tradition européenne, regroupe les différents intérêts des États membres: la transition énergétique en Allemagne et en France, le gaz et les interconnexions en Europe du Sud-Est, les interconnexions en Espagne et en Italie, ainsi que l'innovation en Europe centrale, en Europe du Nord et au Royaume-Uni. Les principaux thèmes de l'Union de l'énergie sont la directive sur les énergies renouvelables, la conception du marché de l'électricité, la politique des transports et la numérisation de l'énergie.

\section{La directive sur les énergies renouvelables}

En ce qui concerne la directive sur les énergies renouvelables, il existe des divergences croissantes entre les États membres quant à l'objectif de $27 \%$ d'énergies renouvelables ou plus d'ici 2030, fixé par l'UE. La question est de savoir si le cadre politique de l'UE peut combattre ces divergences au moyen d'outils permettant de les éviter (gap avoiders) ou de combler les écarts (gap fillers).

Les gap avoiders sont les fonds structurels de l'UE et une institution européenne de conciliation en cas de modifications rétroactives. À mon avis, les modifications rétroactives des lois en Espagne, en Roumanie, en Bulgarie et au Royaume-Uni ont une très mauvaise influence sur la confiance des investisseurs. Il existe encore de nombreuses autres possibilités pour amener les États membres à en faire plus. Par exemple, il est question d'un arbitrage entre les différents instruments : de cette manière, un État membre qui améliore son efficacité énergétique pourrait, en contrepartie, utiliser un peu moins d'énergies renouvelables.

En ce qui concerne les gap fillers, il faut tenir compte du fait que le coût de $1 \%$ en deçà de l'objectif s'élève à 2-2,5 milliards d'euros par an, sur 20 ans.

\section{Conception du marché de l'énergie}

Notre système électrique se caractérise par une forte augmentation de l'intensité capitalistique. Le secteur des énergies renouvelables et l'énergie nucléaire se composent presque exclusivement de capital. Même les centrales conventionnelles sont marquées par une intensité capitalistique toujours plus élevée, parce qu'elles ont une durée d'exploitation de plus en plus courte. Nous supposons qu'en 2030, environ $50 \%$ des centrales conventionnelles seront inutilisées pendant $80 \%$ du temps. Les centrales seront alors payées pour leurs 
capacités et pour leur production d'électricité. La manière dont cela doit fonctionner fait débat.

\section{Politique des transports}

Le domaine des transports va connaître une véritable révolution technologique, motivée par la baisse des coûts technologiques et renforcée par la numérisation. Des études convergentes indiquent qu'entre 2020 et 2030, le coût total de possession (total cost of ownership, TCO) du moteur hybride rechargeable aura rejoint celui du moteur à combustion. C'est un changement technologique de grande ampleur, qui n'est plus automatiquement impulsé par la politique, mais tiré par le marché. Néanmoins, on ne précise pas comment cela fonctionnera et quel sera le rôle de la réglementation européenne.

\section{Numérisation de l'énergie}

Actuellement, la discussion sur la politique énergétique repose sur la sécurité d'approvisionnement, synonyme de pouvoir et d'influence. L'objectif est de garantir l'accès aux ressources. Pour s'affranchir des pays du Golfe, la France mise sur l'énergie nucléaire. Ailleurs, il existe des partenariats énergétiques. Quant aux États-Unis, ils cherchent à peser dans le Golfe afin de garantir leur sphère de pouvoir et d'influence. Si nous passons maintenant à une numérisation ou à un système énergétique reposant sur la technologie, le point critique sera la technologie, plutôt que les ressources. Cela aura d'importantes conséquences sur l'avenir du système énergétique international et sur les négociations climatiques. Mais actuellement, il y a de l'énergie en excès, ce qui fait que sa valeur est faible. La création de valeur ajoutée réside dans la numérisation.

\section{Principaux chantiers}

\section{Européanisation de la transition énergétique}

Actuellement, il existe plusieurs transitions énergétiques nationales qui dépassent progressivement les frontières. Toutefois, les orientations sont très différentes. Je me demande dans quelle mesure nous parviendrons à créer un plan européen.

\section{Investissements}

En ce qui concerne les investissements, le débat en Europe reste très bureaucratique: selon le plan Juncker, les investissements sont entraînés par l'investissement public. Contrairement à il y a 20 ans, il règne aujourd'hui une grande incertitude dans le secteur de l'énergie. En fait, nous ignorons complètement à quoi il ressemblera dans 20, 30 ou 40 ans, et par exemple combien de 
capacités de transport seront nécessaires. Si nous avons un modèle dans lequel nous misons plus sur l'énergie solaire décentralisée, nous n'aurons pas besoin d'un vaste réseau. En raison de cette incertitude, les investissements vont se faire attendre. Aux États-Unis, le capital dans le secteur de l'énergie est du capital-risque. C'est compréhensible, car on ne sait pas à quelle vitesse les véhicules électriques vont s'imposer, ni ce qu'impliquera cette industrie solaire. Il faut encore créer un cadre pour cela.

\section{Compétitivité}

En Europe, nous avons un système composé de quotas gratuits, auxquels s'ajoute une compensation pour les entreprises fortement consommatrices d'énergie. C'est un système qui fonctionne très bien comme solution transitoire, mais pas à long terme, parce qu'aucune entreprise mondiale n'investit si elle dépend de subventions publiques. C'est simplement trop risqué, parce que les gouvernements changent. Les entreprises veulent un cadre structurel dans lequel elles savent effectivement d'où proviennent leurs revenus et s'ils dépendent ou non à $100 \%$ du gouvernement. Les entreprises fortement consommatrices d'énergie ont besoin d'instruments de couverture, par exemple de contrats de fourniture d'électricité à long terme. Actuellement, il y a un débat pour savoir comment nous pouvons distribuer de moins en moins de quotas gratuits à un nombre toujours plus réduit d'entreprises. C'est un véritable frein à l'investissement.

\section{Après 2030}

\section{Échelle européenne ou nationale?}

En ce qui concerne l'après-2030, on se demande tout d'abord comment on peut européaniser les transitions énergétiques. Au plan national, la transition énergétique est peut-être pertinente dans les grands États membres comme la France et l'Allemagne, mais elle n'a naturellement aucun sens pour la Lituanie, la Bulgarie ou le Portugal. Actuellement, nous revenons plutôt à des solutions nationales. Une orientation plus européenne, voire continentale, serait cependant nécessaire pour stimuler l'investissement.

\section{L'approche bureaucratique de l'UE est-elle adéquate?}

Le second point est que l'UE a une approche plutôt bureaucratique de quantification. Des objectifs sont fixés, puis on négocie cette quantification lors de discussions de répartition très compliquées. On procède à un important équilibrage entre les différents États membres au Conseil européen. Cependant, nous n'avons pas encore eu de discussion européenne sur les stratégies à adopter, comme c'est par exemple le cas aux États-Unis ou en Chine. 
Des entreprises high-tech européennes?

Est-il vrai qu'il n'existe pas d'entreprises high-tech européennes, ou bien sontelles cachées dans d'autres entreprises ? Les statistiques du commerce montrent effectivement que l'Europe est toujours le premier exportateur de produits hightech.

La deuxième question brûlante est : est-ce que c'est vraiment important? Dans les années 1980, on s'est beaucoup demandé où était le Compaq européen. Compaq était une société informatique qui a fusionné en 2002 avec HewlettPackard. La grande question était de savoir si l'Europe perdait le contact avec le secteur high-tech parce que nous n'avions pas d'équivalent de Compaq. Dix ans plus tard, les ordinateurs étaient devenus des produits à faibles marges. On se demande donc si la même chose va se produire ou non avec les technologies du secteur climatique. À l'époque, nous savions comment obtenir un Compaq européen. On ne se posait pas la bonne question, or l'un des grands enjeux pour l'Europe sera de se poser les bonnes questions.

Traduction de Marie-Céline GEORG 



\section{Le pentagone du marché de l'électricité : une conception pragmatique du marché de l'électricité pour la transition énergétique européenne ${ }^{1}$}

\section{Philipp LITZ}

Lors du sommet mondial sur le climat qui s'est tenu à Paris en décembre 2015, l'Europe $s^{\prime}$ 'est engagée à atteindre l'objectif mondial de limitation du réchauffement climatique « bien en dessous de $2{ }^{\circ} \mathrm{C}$ par rapport aux niveaux préindustriels » et à " poursuivre les efforts pour limiter la hausse des températures à $1,5^{\circ} \mathrm{C}$ par rapport aux niveaux préindustriels 2 ॥.

Pour respecter l'accord de Paris, l'Europe doit rester dans la fourchette haute des objectifs de décarbonation définis dans la feuille de route à l'horizon 2050 de l'UE. Spécifiquement, d'ici 2050, elle doit réduire ses émissions globales de gaz à effet de serre (GES) de $95 \%$ par rapport aux niveaux de $1990^{3}$. Cela ne sera pas possible sans une décarbonation complète du secteur de l'électricité.

Les choix d'investissement réalisés entre 2015 et 2030 seront cruciaux pour déterminer si le système électrique de l'UE peut connaître une transition en douceur vers la décarbonation totale d'ici 2050. Une transition en douceur nécessite un cadre d'investissement stable. Ce cadre d'investissement, à son tour, initierait un cercle vertueux économique, en créant de nouveaux emplois, en favorisant la croissance et en améliorant la compétitivité.

II existe un risque évident que la transition énergétique européenne soit retardée par d'anciens choix d'investissements, par la dépendance au sentier (path dependency) et par des considérations économiques sorties des manuels qui idéalisent le pouvoir de transformation des marchés. Cumulés, ces risques créeraient de coûteux cycles d'arrêt/redémarrage, des effets de verrouillage et des coûts bien plus élevés que nécessaire pour la transition énergétique. Globalement, cela signifie que l'Europe se priverait des avantages économiques d'une révolution énergétique qu'elle a contribué à créer, laissant aux États-Unis, à la Chine et à certaines économies émergentes en croissance rapide les premiers bénéfices, au détriment de la compétitivité européenne et de sa base industrielle.

Le cadre réglementaire de l'UE, qui détermine les choix d'investissement dans le secteur de l'électricité, peut jouer un rôle clé pour minimiser ces risques. Actuellement, ce cadre s'appuie excessivement sur des principes économiques "d'école ", sans apporter la certitude dont les véritables investisseurs ont besoin, tout en donnant trop peu de résultats, trop tard.

\footnotetext{
${ }^{1}$ Cet article, rédigé en janvier 2017, est une version abrégée de : Agora Energiewende, The Power Market Pentagon - A Pragmatic Power Market Design for Europe's Energy Transition, Berlin, 2016.

${ }^{2}$ Cf. accord de Paris, article 2.1 a).

${ }^{3}$ La tranche $80-95 \%$ a été définie en fonction de l'objectif précédent, contenir le réchauffement climatique à moins de $2{ }^{\circ} \mathrm{C}$. Cf. Commission européenne, Feuille de route vers une économie compétitive à faible intensité de carbone à l'horizon 2050, Bruxelles, 8 mars 2011 [COM(2011) 112 final].
} 
Cet article confronte les approches simplistes actuellement appliquées - spécifiquement celles qui se composent uniquement d'un marché de l'électricité pur (ou " marché energy only ") et d'un système d'échange de quotas d'émission (SEQE) - à une approche pragmatique et constructive, que nous appelons le pentagone du marché de l'électricité. Cette approche vise à maximiser la valeur du marché energy only et du SEQE pendant la période de transition, mais y ajoute trois éléments : (1) des mesures actives pour retirer du système les capacités de production dépourvues de flexibilité et fortement émettrices de carbone; (2) des mesures visant à générer des flux de recettes stables pour les investissements nécessaires dans de nouvelles capacités de production à base d'énergies renouvelables (qui constitueront l'ossature du futur mix énergétique européen totalement décarboné) ; et (3) des mesures pour préserver l'adéquation du système. Dans cet article, nous expliquons comment les différents éléments du pentagone du marché de l'électricité interagissent, et pourquoi cette approche attirera les investissements nécessaires sur la période 2015-2030, tout en maintenant les coûts à un niveau bas et en assurant la fiabilité du système électrique.

\section{L'Europe sur la voie d'un système énergétique basé sur l'éolien, le solaire et la flexibilité}

En décembre 2015, à la conférence de Paris sur le climat, l'UE faisait partie de la «coalition ambitieuse » qui a réussi à faire adopter un objectif global contraignant de stabilisation du réchauffement climatique «bien en dessous de $2{ }^{\circ} \mathrm{C}$ par rapport aux niveaux préindustriels », et un appel à «poursuivre les efforts pour limiter la hausse des températures à $1,5^{\circ} \mathrm{C}$ par rapport aux niveaux préindustriels ${ }^{4} \gg$.

Cela signifie que le secteur de l'électricité devra être totalement décarboné d'ici 2050 au plus tard ${ }^{5,6}$. Le budget carbone restant en 2050 sera nécessaire pour les secteurs dépourvus du potentiel technique permettant une décarbonation économique, comme l'agriculture ou l'industrie lourde. Afin d'atteindre cet objectif, il faudra réaliser environ la moitié des efforts d'ici 2030, ce qui implique d'importants investissements dans les technologies zéro carbone dans les 15 prochaines années.

L'objectif de l'UE à l'horizon 2030 - générer au moins $27 \%$ de son énergie à partir de sources renouvelables - correspond, d'après la Commission européenne, à une part de 45 à $53 \%$ d'énergies renouvelables dans le secteur de l'électricité ${ }^{7}$. La part de l'électricité renouvelable pourrait même être supérieure

\footnotetext{
${ }^{4}$ Cf. accord de Paris, article 2.1 a).

${ }^{5}$ Commission européenne, Impact Assessment Energy Roadmap 2050, Bruxellles, 2011 [SEC(2011) 1565/2].

${ }^{6}$ European Climate Foundation, Roadmap 2050. A Practical Guide to a Prosperous, Low-Carbon Europe, Bruxelles, 2010 ; Commission européenne, Feuille de route pour l'énergie à l'horizon 2050, Bruxelles, 2011 [COM(2011) 885 final].

7 Voir l'analyse d'impact de la Commission sur un cadre réglementaire pour le climat et l'énergie entre 2020 et 2030 (Commission européenne, A Policy Framework for Climate and Energy in the Period from 20202030, Bruxelles, 22/01/2014 [SWD(2014) 15 final]) pour connaître les scénarios compatibles avec une réduction de $40 \%$ des émissions de GES. Il convient de noter que l'investissement nécessaire dans les énergies renouvelables dépend des objectifs d'efficacité énergétique ; cf. Edith Bayer, Efficiency First: Key Points for
} 
si l'Europe rehaussait son ambition globale pour 2030 en phase avec l'accord mondial de Paris sur le climat.

Atteindre une part moyenne annuelle de $50 \%$ ou plus d'électricité provenant de sources d'énergie renouvelable (EnR) constitue un formidable défi. Aujourd'hui, la part des EnR dans la production d'électricité en Europe se situe autour de $26 \%{ }^{8}$. Cela signifie que dans les 15 prochaines années, il faudra approximativement doubler cette part dans les systèmes électriques de toute l'Europe.

D'après les tendances actuelles, ce sont les installations photovoltaïques et l'éolien terrestre qui représenteront de loin la majeure partie des nouvelles capacités de production d'énergie renouvelable ${ }^{9}$. En un mot, ces deux technologies ont gagné la course à la réduction des coûts technologiques. De plus, on attend encore d'importantes baisses des coûts, en particulier pour le photovoltaïque ${ }^{10}$.

Cependant, les éoliennes et les panneaux photovoltaïques ne produisent d'électricité que lorsque le vent souffle et que le soleil brille. Ce sont donc des sources d'électricité fluctuantes, qui ne peuvent pas simplement remplacer les infrastructures de charge de base selon un principe strictement proportionnel. La transition vers une part bien plus importante d'EnR fluctuantes aura donc des répercussions sur tout le système: de grandes centrales de production d'électricité feront place à un nombre croissant de technologies décentralisées zéro carbone. Cette évolution aura, à son tour, des conséquences sur les investissements planifiés dans l'infrastructure de réseau, au niveau du transport comme à celui de la distribution. Dans l'ensemble, les systèmes électriques devront devenir plus flexibles, à la fois en termes d'offres d'approvisionnement et de satisfaction de la demande.

En outre, l'essentiel du parc de centrales d'Europe a été construit avant la libéralisation et l'intégration transfrontalière des marchés électriques européens. Aujourd'hui, plus de $70 \%$ des centrales électriques conventionnelles en Europe ont plus de 30 ans. En considérant une durée de vie prévue de 40 ans pour les centrales à gaz, 40-50 ans pour les centrales à houille et 50 ans pour les cen-

the Energy Union Communication [en ligne], Regulatory Assistance Project (RAP), Bruxelles, 2015. Disponible sur: http://www.raponline.org/knowledge-center/efficiency-first-key-points-for-the-energy-union-com munication/ [consulté le 14/03/2017].

${ }^{8}$ Commission européenne, Rapport sur les progrès accomplis dans le secteur des énergies renouvelables, Bruxelles, 2015 [COM(2015) 293 final].

${ }^{9}$ Depuis l'an 2000, 443 GW de nouvelles capacités de production ont été installés en Europe, dont $58 \%$ d'EnR, principalement éolienne et solaire (European Wind Energy Association, Wind in Power: 2015 European Statistics, Bruxelles, 2016). À l'horizon 2050, les scénarios inclus dans la feuille de route de l'UE sur l'énergie montrent que la part de l'énergie éolienne et du photovoltaïque pourrait atteindre $67 \%$ du mix électrique (Commission européenne, Impact Assessment Energy Roadmap 2050, Bruxelles, 2011 [SEC(2011) $1565 / 2]$ ). Cette tendance vaut non seulement pour l'Europe, mais aussi pour le reste du monde (cf. par exemple International Renewable Energy Agency (IRENA), Renewable Energy Capacity Statistics 2015, Masdar City, 2016).

${ }^{10}$ Fraunhofer-Institut für Solare Energiesysteme ISE, Current and Future Cost of Photovoltaics. Long-Term Scenarios for Market Development, System Prices and LCOE of Utility-Scale PV Systems, Fribourg-enBrisgau, 2015 [Étude réalisée pour le compte d'Agora Energiewende]. 
trales au lignite et les centrales nucléaires, cela signifie qu'une grande partie du parc européen arrivera en fin de vie dans les 15 prochaines années. Bien avant l'expiration de ce délai, c'est-à-dire essentiellement dans la décennie à venir, les entreprises de services publics devront décider si elles veulent moderniser ces centrales, les remplacer par des capacités de production à émissions de carbone faibles ou nulles, ou investir dans l'usage intelligent et efficace d'installations de moindre capacité.

En prenant ces deux aspects en compte, d'un point de vue décisionnel, il est donc économiquement pertinent d'assouplir le système électrique en améliorant la flexibilité du parc européen de centrales électriques, tout en mettant en service une part croissante de capacités renouvelables variables. Dans le cas contraire, si l'augmentation de la part des énergies renouvelables ne s'accompagne pas d'une évolution du système vers un bouquet de capacités de production plus flexible et qualitativement différent, les conséquences économiques seront négatives pour la société.

\section{Pourquoi il ne suffit pas de renforcer le marché energy only et le SEQE pour réussir la transition énergétique dans l'UE}

Les partisans d'une approche harmonisée de la politique climatique et énergétique dans l'UE affirment que la transition énergétique européenne devrait reposer sur deux éléments principaux : un marché de l'électricité pur renforcé et un système d'échange de quotas d'émission (SEQE) renforcé. Ils affirment que ces deux instruments offrent le moyen le plus rentable pour effectuer une transition fiable vers un système énergétique à faibles émissions de carbone. De plus, les instruments additionnels perturbent le fonctionnement efficace des marchés et devraient être éliminés le plus vite possible.

Nous allons maintenant présenter la base théorique de ces opinions. Nous montrerons ensuite pourquoi, parce qu'elles reposent sur des principes économiques simplistes et idéalisés ${ }^{11}$, ce sont des réflexions à courte vue. Nous pensons que la foi dans cette approche présente un très haut risque de dérapage dans la progression vers une décarbonation rentable, ce qui aurait des répercussions négatives graves sur le leadership industriel et technologique de l'Europe ainsi qu'un impact négatif sur l'emploi, la croissance et la compétitivité.

\section{Marché energy only et SEQE : des principes économiques purement livresques}

On ne manque pas de littérature sur le fonctionnement des marchés énergétiques purs et sur la bonne conception des systèmes d'échange de quotas d'émission ${ }^{12}$.

\footnotetext{
${ }^{11}$ Ces modèles reposent notamment sur les hypothèses suivantes : une anticipation sans faille, une demande élastique par rapport au prix et une concurrence parfaite, ainsi que l'internalisation complète du coût externe des émissions de carbone.

${ }^{12} \mathrm{Au}$ sujet des marchés energy only, voir par exemple : Michael Caramanis, «Investment Decisions and Long-Term Planning Under Electricity Spot Pricing », IEEE Transactions on Power Apparatus and Systems,
} 
Toutefois, au moins dans l'UE, la combinaison du marché energy only et du SEQE s'est montrée jusqu'à présent incapable de stimuler l'investissement dans un système énergétique d'avenir, sans carbone et diversifié ${ }^{13}$. Afin de comprendre les raisons de cet échec, il semble utile de commencer par exposer les principes théoriques de ces affirmations.

Affirmation 1 : en l'absence de distorsion, les marchés energy only assurent suffisamment de revenus et d'incitations pour de nouveaux investissements dans tous les types de technologies de production d'électricité et de réponse à la demande.

La conclusion théorique que les marchés energy only assurent suffisamment de revenus pour les nouveaux investissements ne tient que dans certaines conditions et sous certaines hypothèses. Premièrement, la demande doit être élastique par rapport au prix, c'est-à-dire que les consommateurs d'électricité doivent réduire leur consommation lorsque les prix augmentent sur le marché de l'électricité. Spécifiquement, une demande élastique par rapport au prix facilite l'équilibre du marché (c'est-à-dire la correspondance entre l'offre et la demande) lorsque l'offre est saturée, en générant ce qu'on appelle un prix de pénurie. Les consommateurs disposés à payer moins que le prix d'équilibre du marché vont retarder ou réduire leur consommation d'électricité à ces momentslà, ce qui permet d'éviter les délestages involontaires (baisses de tension, coupures localisées). En retour, les prix atteindront des niveaux plutôt élevés pendant ces quelques heures, ce qui facilitera le recouvrement total des coûts pour toutes les technologies. En plus de l'élasticité de la demande par rapport au prix, il faut remplir les conditions d'une anticipation sans faille et d'une concurrence parfaite pour que le marché energy only donne des résultats efficaces ${ }^{14}$. Dans ces conditions, il est possible d'éviter les cycles d'expansion-récession (alternances répétées de surinvestissement et sous-investissement).

En théorie, les investissements dans des centrales dites «de pointe » sont vitaux. En effet, ces centrales fonctionnent pendant quelques heures seulement, à des moments où la consommation est élevée et la production d'énergies renouvelables faible. Or les centrales de pointe nécessitent des prix de l'électricité élevés (prix dits de pénurie) pendant leurs (rares) heures de fonctionnement afin de permettre un recouvrement total des coûts (y compris les investissements initiaux).

À l'avenir, l'électricité sera principalement produite par des actifs renouvelables et à faibles émissions de carbone. Quelle que soit la combinaison technologique spécifique, cela représentera un défi supplémentaire parce que les

vol. 101, n 12, 1982, p. 4640-4648; Michael Crew, Chitru Fernando, Paul Kleindorfer, « A Theory of Peak Load Pricing: A Survey », Journal of Regulatory Economics, n ${ }^{8}$, 1995, p. 215-248.

${ }^{13}$ Les derniers chiffres de Bloomberg New Energy Finance (BNEF) montrent une baisse des investissements dans les énergies renouvelables uniquement en Europe, et une croissance parfois forte ailleurs dans le monde (BNEF, Clean Energy Investment: Q4 2015 Factpack, New York, 2016).

${ }^{14}$ Laurens de Vries, The Instability of Competitive Energy-Only Electricity Markets, [s.e.], [s.1.], 2003. 
énergies renouvelables, spécifiquement l'éolien et le photovoltaïque, se caractérisent par des coûts d'investissement relativement élevés et des coûts marginaux relativement bas, voire nuls. Ces sources sont généralement en fonctionnement lorsque les prix de gros sont bas, et elles ne tirent parti de prix élevés que de manière très limitée. Elles sont donc plus vulnérables que les sources conventionnelles aux prix de pénurie stochastiques.

En théorie, l'absence de distorsion sur les marchés de l'électricité devrait assurer le recouvrement total des coûts pour les technologies renouvelables, pour les technologies d'approvisionnement résiduel à faibles émissions de carbone et pour les adaptations de la demande, à condition que le SEQE définisse un prix du carbone suffisamment élevé pour refléter l'importance des réductions d'émissions nécessaires.

Affirmation 2: les systèmes d'échange de quotas d'émission peuvent favoriser une décarbonation rentable du système électrique en définissant un plafond d'émissions contraignant et décroissant.

Selon les théories livresques, le marché energy only orientera également les investissements vers les options à émissions de carbone faibles ou nulles, à condition que le coût de ces émissions, auparavant externalisé, soit internalisé ${ }^{15}$. Le montant de ce surcoût reflète le niveau « socialement responsable » d'émissions de carbone qui est possible pour les secteurs économiques relevant du système d'échange de quotas. Idéalement, ce plafond est cohérent avec la réduction des émissions à long terme nécessaire pour atteindre les objectifs à long terme de lutte contre le réchauffement climatique.

Le plafond d'émissions déclenche une pénurie de quotas d'émission, ce qui entraîne la fixation d'un prix pour les certificats d'émission, qui à son tour encourage les mesures de réduction (lesquelles ont un coût). Le prix des certificats va pousser le marché à privilégier les technologies à faibles émissions de carbone par rapport aux technologies fortement émettrices et, théoriquement, faciliter une réduction rentable des émissions de $\mathrm{CO}_{2}$, puisque les investissements seront réalisés là où le coût marginal de réduction d'une quantité donnée d'émissions sera le plus faible.

Le prix des certificats orientera la répartition des ressources existantes, en favorisant l'usage accru des centrales à faibles émissions de carbone tout en encourageant l'investissement dans les nouvelles technologies à faibles émissions de carbone ainsi que la fermeture des infrastructures très polluantes. De fait, le SEQE devrait permettre la transition des installations conventionnelles fortement émettrices vers les moins émettrices, et des sources fossiles vers les énergies renouvelables zéro carbone.

\footnotetext{
${ }^{15}$ Ce prix peut résulter d'un système d'échange de quotas d'émission ou d'une taxe carbone. Ci-après, nous nous référerons uniquement au SEQE. Il convient de noter que la théorie définit un prix du carbone dans tous les secteurs.
} 
Comme le marché energy only, le SEQE théorique repose sur la certitude des acteurs du marché : ils doivent avoir confiance dans la stabilité du cadre réglementaire et dans le fait que le plafond d'émissions va progressivement baisser.

\section{Les failles du marché energy only et du SEQE dans le monde réel}

Les chiffres récents sur les investissements mondiaux dans les énergies renouvelables montrent des niveaux en baisse en Europe, tandis qu'ils augmentent partout ailleurs ${ }^{16}$. Ce fait, à lui seul, devrait nous encourager à considérer honnêtement les mérites et les failles du marché energy only et du SEQE en tant qu'outils de la décarbonation en Europe.

À notre avis, le fait de s'appuyer sur des solutions inspirées de principes économiques simplistes causera certainement l'échec des efforts de décarbonation, et ce pour au moins six raisons :

- la dépendance au sentier : les marchés sont des constructions sociales. Dans la vie réelle, ils reflètent les imperfections et les contraintes des trajectoires imposées par les choix antérieurs ${ }^{17}$. L'évolution historique du mix énergétique dans les États membres est l'une des contraintes spécifiques qui limitent l'action de la politique climatique et énergétique en Europe. En matière de système énergétique, les choix du passé ont été effectués sans tenir compte d'objectifs climatiques ${ }^{18}$. De plus, les marchés de l'électricité dans l'UE n'ont été que partiellement libéralisés et intégrés ${ }^{19}$. Par conséquent, le système électrique actuel dans la plupart des pays d'Europe se caractérise par un excès de capacités de production et une part importante d'infrastructures de charge de base dépourvues de flexibilité. Ces deux aspects constituent un défi majeur pour la transition vers un système énergétique de marché ;

- l'incertitude : la logique théorique des investissements déterminés par le marché energy only et le SEQE néglige une caractéristique importante des marchés réels, l'incertitude. Un certain niveau d'incertitude est tout simplement une donnée dans tout marché, et ne représente pas forcément un inconvénient. Cependant, en fonction de la nature de l'incertitude, celle-ci peut constituer un obstacle dans des marchés supposés contribuer à atteindre des objectifs politiques, comme c'est le cas des marchés de l'énergie et du carbone en Europe. L'incertitude se tra-

\footnotetext{
${ }^{16}$ BNEF, Clean Energy Investment: Q4 2015 Factpack, New York, 2016.

${ }^{17}$ Cf. l'ouvrage précurseur de Douglass C. North : Institutions, Institutional Change and Economic Performance, Cambridge University Press, Cambridge, 1990.

${ }^{18}$ Par exemple, le charbon a été crucial pour la révolution industrielle en Allemagne, au Royaume-Uni et dans d'autres pays.

19 Agency for the Cooperation of Energy Regulators (ACER)/Council of European Energy Regulators (CEER), ACER/CEER - Annual Report on the Results of Monitoring the Internal Electricity and Natural Gas Markets in 2014, Ljubljana/Bruxelles, 2015.
} 
duit par un risque lorsque l'impact économique d'événements incertains peut être calculé. La gestion des risques est une activité économique de base. Du point de vue des acteurs du marché, il y a des risques contre lesquels on peut se prémunir à l'intérieur du cadre existant, et d'autres contre lesquels il est impossible de se protéger (par exemple, les changements futurs des règles du marché), ou bien à un coût prohibitif ;

- le risque réglementaire : un système électrique fiable et sûr est d'une grande importance pour toute économie. Pour cette raison, la fiabilité du système électrique est souvent considérée comme un bien public. Même si, théoriquement, un marché energy only peut encourager de nouveaux investissements et assurer la fiabilité du système, de nombreux responsables politiques et organes de régulation semblent douter de l'efficacité du marché energy only. En pratique, la baisse des marges de réserve des systèmes électriques a déclenché un débat sur la nécessité de favoriser des investissements supplémentaires pour «garder les lumières allumées », que ce soit par des marchés de capacité à part entière ou des approches «filet de sécurité » telles que des réserves de capacité ou des réserves stratégiques ${ }^{20}$. Dans la plupart des États membres de l'UE, un ou plusieurs de ces instruments ont déjà été mis en œuvre. L'introduction de ces instruments et le débat public dont ils font l'objet ont accru l'incertitude chez les acteurs du marché, ce qui a réduit les probabilités d'investissements de marché dans de nouvelles capacités ;

- la valeur de marché de l'éolien et du photovoltaïque : le milieu scientifique est engagé dans un débat intense concernant les prix de marché de l'électricité que peuvent atteindre les installations à base d'EnR pendant les heures auxquelles elles produisent, lorsque le système électrique dispose d'une forte proportion d'énergies renouvelables variables ${ }^{21}$. Audelà des arguments théoriques, il semble qu'une part accrue d'énergies renouvelables variables est associée à une chute des revenus de marché pour chaque kilowattheure d'électricité produite par ces EnR variables. De plus, lorsque la part des EnR dans la production électrique est élevée, le prix marginal du marché de gros va être de plus en plus souvent déterminé par l'électricité renouvelable et nucléaire, et non par les centrales à combustibles fossiles relevant du SEQE. Pendant ces heures, le SEQE ne contribuera donc pas au prix de marché obtenu par les producteurs d'électricité à base d'EnR ;

\footnotetext{
${ }^{20}$ Les réserves de capacité ou les réserves stratégiques répondent aux inquiétudes politiques sur l'aptitude du marché energy only à construire des capacités suffisantes. Elles ne réduisent pas les risques que les capacités restantes au sein du marché energy only puissent générer un risque de dérive : au fil du temps, la taille des réserves devient de plus en plus importante à cause du manque d'investissements déterminés par le marché.

${ }^{21}$ Cf., par exemple, Agora Energiewende, The Integration Cost of Wind and Solar Power, Berlin, 2015, et les références qui y figurent; Lion Hirth, «The Market Value of Variable Renewables », Energy Economics, $\mathrm{n}^{\circ} 38,2013$, p. 218-236 ; Michael Hartner et al., « East to West - The Optimal Tilt Angle and Orientation of Photovoltaic Panels from an Electricity System Perspective », Applied Energy, vol. 160, 2015, p. 94-107.
} 
- l'offre excédentaire dans le SEQE : depuis plusieurs années, le SEQE connaît systématiquement un excédent d'offre. Le surplus actuel s'établit à environ 2 milliards de quotas d'émission. Dans ce contexte, les législateurs européens ont récemment modifié le SEQE en créant une « réserve de stabilité de marché » $(\mathrm{RSM})^{22}$. L'objectif principal de cette RSM est de mieux protéger le SEQE contre les chocs externes tels qu'une crise économique. Cependant, les projections suggèrent que la RSM sera incapable de réduire substantiellement l'offre excédentaire avant la fin des années 2020. Ainsi, même s'il s'agit d'un instrument économiquement efficace en théorie, dans le monde réel, le SEQE donnera des résultats trop faibles et trop tardifs, malgré les récentes propositions de réforme ;

- la non-acceptation de prix du $\mathrm{CO}_{2}$ suffisamment élevés : une réduction plus rapide et plus radicale de l'excédent d'offre pourrait faire augmenter les prix plus vite. Cependant, considérant l'historique du SEQE à ce jour, et étant donné l'anxiété des décideurs politiques vis-à-vis des risques de fuite de carbone ou les inquiétudes sur une possible désindustrialisation de l'Europe, il est peu probable que l'on atteigne des prix de quotas suffisamment élevés dans un avenir proche.

\section{Le pentagone du marché de l'électricité}

Dans ce contexte de dépendance au sentier, d'incertitudes fondamentales sur la dynamique du marché, et de signaux d'investissements insuffisants émanant du SEQE, nous envisageons cinq éléments (détaillés ci-dessous) comme des parties importantes d'un marché de l'électricité post-2020 cohérent avec le triple objectif de décarbonation totale du système électrique d'ici 2050, tout en garantissant la sécurité de l'approvisionnement et un coût de transition réduit. Ensemble, ces cinq éléments forment le pentagone du marché de l'électricité. Nous considérons ici ce pentagone comme faisant partie intégrante du premier principe de «l'efficacité énergétique», un principe systématique consistant à donner la priorité aux investissements dans les ressources génératrices d'efficacité à chaque fois qu'elles coûteraient moins cher ou rapporteraient plus que l'investissement dans toute autre source de fourniture d'énergie ou infrastructure disponible ${ }^{23}$.

\footnotetext{
${ }^{22}$ Parlement européen, Conseil de l'Union européenne, « Décision (UE) 2015/1814 du Parlement européen et du Conseil du 6 octobre 2015 concernant la création et le fonctionnement d'une réserve de stabilité du marché pour le système d'échange de quotas d'émission de gaz à effet de serre de l'Union et modifiant la directive 2003/87/CE », Journal officiel de l'Union européenne, L 264, 9 octobre 2015, p 1-5.

${ }^{23}$ Edith Bayer, Efficiency First: Key Points for the Energy Union Communication [en ligne], Regulatory Assistance Project (RAP), Bruxelles, 2015. Disponible sur: http://www.raponline.org/knowledgecenter/efficiency-first-key-points-for-the-energy-union-communication/ [consulté le 14/03/2017].
} 
Élément 1 : les marchés de l'électricité purs et les marchés d'équilibre gèrent le défi de la flexibilité en pilotant l'offre et la demande

Actuellement, les marchés de l'électricité en Europe sont des marchés énergétiques purs. Les fournisseurs et les consommateurs échangent des kilowattheures, c'est-à-dire des quantités spécifiques d'énergie à un moment donné. Le prix de l'électricité sur les marchés de gros est déterminé en triant les offres de production de la moins chère à la plus chère, et en les croisant avec les enchères de demande. Avec ce mécanisme de classement «par ordre de mérite», les centrales électriques ayant les coûts de fonctionnement les plus faibles sont employées d'abord, puis celles dont le coût de fonctionnement est plus élevé. Tant qu'il existe un surplus de production, les prix correspondent au coût de fonctionnement (ou au coût marginal) de la centrale la plus chère en service dans le système.

Pour relever les défis du monde réel sur les marchés actuels de l'électricité en transition, il est crucial d'adapter ces marchés à la flexibilité. Pour cela il faudrait :

- coupler les marchés de l'énergie et les rendre plus rapides pour améliorer l'efficacité de la flexibilité ;

- relier des segments de marché à court terme pour atteindre des prix de marché reflétant la valeur de l'énergie en temps réel ;

- améliorer la prédictibilité des prix de pénurie pour réduire les risques, soutenir des investissements efficaces et contribuer à la fiabilité ;

- créer des règles du jeu équitables pour la flexibilité du côté de la demande et de l'offre afin de réduire les coûts totaux et de maximiser la fiabilité.

Bien que les changements de conception du marché décrits ci-dessus soient des préalables importants à un système énergétique plus flexible et plus rentable, ils n'auront d'effet que si les prix du marché de l'électricité sont suffisamment élevés pour rendre économiquement valables les options de flexibilité du côté de l'offre ou de la demande. À l'heure actuelle, les prix de gros du marché de l'électricité en Europe sont inhibés par les surcapacités et la part élevée d'infrastructures de charge de base sans flexibilité, utilisant du charbon bon marché $^{24}$. La mise hors service active des centrales anciennes, non flexibles et fortement émettrices de carbone devrait donc être un élément important de la conception du marché post-2020 (cf. élément 3 ci-après). La valeur de marché des capacités de production flexibles et peu émettrices serait en outre accrue par le renforcement du SEQE (cf. élément 2).

\footnotetext{
${ }^{24}$ Matthias Buck, Michael Hogan, Christian Redl, The Market Design Initiative and Path Dependency: Smart Retirement of Old, High-Carbon, Inflexible Capacity as a Prerequisite for a Successful Market Design, Agora Energiewende/RAP, Berlin/Bruxelles, 2015.
} 
Élément 2 : le SEQE devrait fournir un prix moyen stable du carbone, et interagir intelligemment avec les politiques relatives aux énergies renouvelables, à l'efficacité et au retrait ciblé d'anciennes centrales

Le SEQE est souvent considéré comme «l'outil phare » de la politique climatique européenne ${ }^{25}$. Son importance est mise en évidence par le simple fait qu'il couvre $45 \%$ des émissions de gaz à effet de serre de l'UE. Cependant, comme nous l'avons montré plus haut, le SEQE donne actuellement de mauvais résultats et ne fournira probablement pas un signal prix suffisant et stable pour les investissements dans des capacités à faibles émissions ou zéro carbone avant au moins 2030.

Ces dernières années, les recherches sur les différentes politiques climatiques $^{26}$ ont montré que les systèmes d'échange de quotas d'émission ne devraient pas être considérés comme la «solution universelle» qui disqualifie tous les autres instruments de politique climatique (comme les manuels d'économie le suggéreraient). Ils devraient plutôt être vus comme un outil de politique climatique qui doit être complété par d'autres instruments, comme les normes sur l'efficacité énergétique ou une réglementation sur la mise hors service des installations à fortes émissions de carbone.

Ainsi, le rôle du SEQE dans les politiques climatiques européennes devrait être redéfini comme suit :

- le SEQE n'est pas le bon instrument pour favoriser des installations zéro carbone comme les énergies renouvelables, ni pour éliminer totalement du système les centrales au lignite fortement émettrices, car cela nécessiterait actuellement des prix du carbone supérieurs à 60 euros la tonne de $\mathrm{CO}_{2}$, ce qui ne semble ni réaliste politiquement ni souhaitable, au vu des effets négatifs potentiels d'un tel prix sur une industrie à haute intensité énergétique ;

- le principal rôle du SEQE dans le secteur de l'énergie devrait être de fournir un prix pertinent du carbone facilitant la transition du mix énergétique des combustibles fossiles fortement émetteurs vers des combustibles fossiles émettant moins de carbone. Au prix actuel du charbon et du gaz, un prix du $\mathrm{CO}_{2}$ autour de 30 euros la tonne devrait suffire à atteindre cet objectif ;

\footnotetext{
${ }^{25}$ Commission européenne, « Transformer le système énergétique européen — Le paquet d'été de la Commission européenne ouvre la voie » [en ligne], Commission européenne - Base de données des communiqués de presse, $\mathrm{n}^{\circ} \mathrm{IP} / 15 / 5358,15$ juillet 2015, 3 p. Disponible sur : http://europa.eu/rapid/press-release_IP-155358_fr.htm [consulté le 14/03/2017].

${ }^{26}$ OCDE/Banque mondiale, The FASTER Principles for Successful Carbon Pricing, Paris/Washington, 2015 ; Christina Hood, Managing Interactions Between Carbon Pricing and Existing Energy Policies: Guidance for Policymakers, Agence internationale de l'énergie, Paris, 2013 ; Richard Schmalensee, Robert Stavins, Lessons Learned from Three Decades of Experience With Cap-and-Trade, Harvard Kennedy School, Cambridge, 2015 (Faculty Research Working Paper Series); Felix Matthes, Greenhouse Gas Emissions Trading and Complementary Policies. Developing a Smart Mix for Ambitious Climate Policies, Öko-Institut, Berlin, 2010.
} 
- le plafond du SEQE devrait s'articuler intelligemment avec les objectifs de réduction des émissions de carbone visés par les autres instruments de politique climatique qui affectent le secteur du SEQE, c'est-à-dire les tarifs de rachat, les mesures d'efficacité énergétique et les instruments de retrait ciblé d'anciennes centrales. Ainsi, toute réduction des émissions de $\mathrm{CO}_{2}$ dépassant les prévisions doit être déduite du plafond afin de préserver l'efficacité économique et l'intégrité environnementale de ce plafond ;

- en tant qu'instrument agissant au niveau de l'UE, le SEQE devrait interagir avec les instruments nationaux de politique climatique et favoriser leur action. Afin d'avoir un effet positif sur le climat mondial, les réductions d'émissions supplémentaires venant de ces actions nationales doivent se traduire dans le SEQE par une réduction du plafond global.

\section{Élément 3 : retrait ciblé - mise hors service active des anciennes centrales dépourvues de flexibilité et fortement émettrices de carbone}

La transition énergétique européenne nécessite le déploiement à grande échelle d'énergies renouvelables. Mais pour qu'elle soit rentable, l'adoption des énergies renouvelables doit aller de pair avec une transition plus large du système vers un bouquet de capacités de production beaucoup plus flexible et qualitativement différent. Pour que l'Europe puisse réussir une décarbonation rentable, il faudra une volonté politique et l'engagement à s'affranchir de la dépendance au sentier créée par les investissements antérieurs dans des capacités de production dépourvues de flexibilité et fortement polluantes ${ }^{27}$.

Étant donné que chaque État membre a le droit de déterminer son propre mix énergétique, c'est aux gouvernements nationaux qu'il revient principalement de mettre en œuvre une politique active de retrait des anciennes centrales non flexibles et fortement polluantes. Après l'accord historique sur le climat adopté à Paris, plusieurs États membres ont envisagé d'accélérer leur sortie du charbon, notamment le Royaume-Uni, l'Allemagne, les Pays-Bas et le Danemark.

Toutefois, ces réflexions doivent impérativement envisager la manière dont les politiques nationales s'articulent avec les règles applicables au niveau européen, et comment elles pourraient être complétées utilement par une action au plan européen. Dans la sous-partie précédente sur le SEQE, nous avons déjà expliqué comment la possibilité d'un mécanisme d'annulation des excédents de quotas d'émission résultant des actions nationales supplémentaires accroîtrait la capacité de ces initiatives à apporter une contribution no-

\footnotetext{
${ }^{27}$ Matthias Buck, Michael Hogan, Christian Redl, The Market Design Initiative and Path Dependency: Smart Retirement of Old, High-Carbon, Inflexible Capacity as a Prerequisite for a Successful Market Design, Agora Energiewende/RAP, Berlin/Bruxelles, 2015.
} 
table à la protection du climat. Cependant, le retrait actif des anciennes centrales de charge de base, non flexibles et fortement émettrices de carbone, faciliterait l'intégration d'une part accrue d'énergies renouvelables dans le système électrique, ce qui rendrait plus efficaces les réformes portant sur la conception du marché présentées ci-dessus, tout en contribuant à maintenir la transition énergétique européenne sur une trajectoire rentable en termes de coût du système.

\section{Élément 4 : assurer des revenus de marché stables}

pour les nouveaux investissements dans l'électricité à base d'EnR

Dans les discussions sur le cadre climatique et énergétique à l'horizon 2030, on affirme fréquemment que d'ici 2020, les technologies renouvelables matures seront capables d'être économiquement autonomes et généreront suffisamment de revenus sur le marché de l'électricité pur. Comme nous l'avons montré, une analyse des réalités du marché de l'électricité contredit cette opinion.

C'est pourquoi nous considérons que le dogme selon lequel les énergies renouvelables n'auront plus besoin de soutien après 2020 n'est pas raisonnable, d'un point de vue analytique et politique. En outre, il est également inutile de prédire un financement des EnR par le marché après 2020, car l'évolution vers un système d'appels d'offres concurrentiels pour les nouvelles capacités de production à base d'EnR signifie que les marchés montreront quand et où les conditions d'une rentabilité de marché totale des investissements dans les projets d'énergies renouvelables seront remplies, et où une certaine stabilisation des revenus sera nécessaire et dans quelle mesure.

Le cadre européen de lois, règlements et mesures relatifs à l'énergie renouvelable est important afin de donner aux investisseurs assez de confiance et de stabilité pour qu'ils investissent dans les capacités de production à base d'EnR en Europe. Une situation stable et fiable est considérée comme un risque moindre, ce qui se traduit par un coût moindre pour les promoteurs de projets et un plus faible taux de rentabilité nécessaire pour amortir les investissements. Ce dernier point signifie que, pour le même prix de l'électricité, les projets à faible risque auront besoin d'une aide moins élevée ou nulle pour combler les éventuelles lacunes de revenus.

\section{Élément 5 : préserver l'adéquation du système}

Le faible niveau des prix de gros et les revenus insuffisants pour tous les types de ressources sont souvent considérés comme des signes annonciateurs d'une pénurie de capacités plutôt que d'une réduction bienvenue des surcapacités. C'est une inquiétude majeure dans les débats sur les moyens de préserver l'adéquation du système.

Comme nous l'avons montré, la transition énergétique européenne doit s'appuyer sur une combinaison toujours plus flexible de ressources afin de 
suivre une trajectoire rentable. Préserver l'adéquation du système va devenir de plus en plus une question évolutive : il ne s'agit plus seulement de combien de capacités on a besoin, mais de quel type de capacités.

Pour démontrer la nécessité de mesures destinées à préserver l'adéquation du système :

- un pilier de cet élément est le fait que l'adéquation du système est évaluée au niveau régional transfrontalier pour des raisons de rentabilité et de fiabilité ;

- en plus des efforts constants pour améliorer la méthodologie des évaluations d'adéquation du système au plan régional, la régionalisation de cette surveillance doit faire l'objet d'un débat pour éviter les préjugés potentiels dans les perspectives nationales ;

- enfin, il devrait être impératif d'évaluer l'adéquation du système à une échelle régionale avant d'introduire des programmes de subventionnement des capacités de production nationales.

LES OBJECTIFS DE L'UE EN MATIÈRE DE LUTTE CONTRE le réchauffement climatique exigent une décarbonation totale du secteur électrique européen d'ici 2050 au plus tard. Surtout, la réussite du sommet sur le climat à Paris en décembre 2015 démontre que l'Europe n'est pas seule dans cette entreprise.

Comme nous l'avons montré dans cet article, l'énergie éolienne et le solaire photovoltaïque assureront l'ossature du futur système énergétique zéro carbone de l'UE. La raison en est évidente : même en incluant leur coût d'intégration, ces sources d'énergie sont moins chères que toute autre nouvelle technologie zéro carbone. Pour que l'intégration progressive des parts croissantes d'électricité renouvelable fluctuante soit rentable, le système électrique doit réagir avec flexibilité, du côté de l'offre et de la demande, aux schémas plus variables de production de l'électricité éolienne et photovoltaïque.

Les recherches menées sur une trajectoire de transition rentable montrent qu'il est possible d'accroître la flexibilité du système électrique à des coûts nettement inférieurs, si la part des ressources non flexibles décroît pendant que celle des ressources flexibles augmente.

Cette conclusion simple met en lumière les signaux d'investissement donnés par les marchés de l'électricité en Europe. Les données statistiques sur l'évolution du mix de production européen et les chiffres récents sur les schémas d'investissement dans les énergies renouvelables suggèrent que l'approche actuelle de l'UE vers une décarbonation axée sur le marché ne fonctionne pas comme elle le devrait. Une grande partie des défauts du cadre actuel sont imputables à des solutions reposant sur une approche économique simpliste, composée d'un marché energy only et du SEQE. Dans cet article, nous avons expliqué les hypothèses essentielles qui sous-tendent cette approche et démontré pourquoi elle ne donnera que des résultats trop faibles et trop tardifs. 
À l'opposé, nous proposons de bâtir la transition énergétique européenne sur un pentagone du marché de l'électricité, à partir du premier principe de l'efficacité énergétique. Cette approche pragmatique et constructive pourrait effectivement maximiser la valeur du marché energy only et du SEQE existant, mais en lui ajoutant trois éléments : (i) le retrait ciblé des anciennes centrales dépourvues de flexibilité et polluantes, (ii) des mesures assurant des revenus de marché stables pour les nouveaux investissements dans les EnR, et (iii) des mesures pour préserver l'adéquation du système.

Traduction de Marie-Céline GEORG 

$-\mathrm{VI}$ -

DES STRATÉGIES ÉNERGÉTIQUES

DIFFÉRENCIÉES SELON LES PAYS 



\section{EXPOSÉ INTRODUCTIF}

\section{Transition(s) énergétique(s) en Europe : unis dans la diversité ? ${ }^{1}$}

\section{Thomas PELLERIN-CARLIN}

Le projet d'Union européenne de l'énergie vise à accélérer la transition énergétique en Europe grâce à une coopération européenne plus intense en ces domaines. L'idée de cette Union de l'énergie a été initialement promue dès 2010 par Jacques Delors, ancien président de la Commission européenne et président-fondateur de l'Institut Jacques Delors, et Jerzy Buzek, alors président du Parlement européen. Quatre ans plus tard, l'idée d'Union de l'énergie est reprise par Donald Tusk et Jean-Claude Juncker, tandis que I'Institut Jacques Delors publie un rapport de référence ${ }^{2}$ sur le contenu à donner à cette Union de l'énergie.

La situation énergétique actuelle en Europe est connue : $75 \%$ du mix énergétique primaire de l'Union européenne est constitué d'énergies fossiles avec, par ordre d'importance, le pétrole, le gaz et le charbon. Les États européens se sont par ailleurs dotés, aux niveaux national, européen et international, d'objectifs pour leur politique énergétique. On recense trois objectifs :

- décarboner. Dans le prolongement des stratégies nationales et européenne de réduction des émissions de gaz à effet de serre, l'Union européenne et ses États membres ont signé l'accord de Paris, dont l'article 4 prévoit notamment des émissions nettes de gaz à effet de serre égales à zéro au niveau mondial, d'ici la fin de ce siècle ;

- sécuriser l'approvisionnement en énergie, notamment pour assurer la résilience de nos systèmes énergétiques à une potentielle perturbation de nos approvisionnements en gaz russe, pétrole du Moyen-Orient ou à d'éventuelles coupures massives de courant en Europe ;

- assurer la compétitivité. Il s'agit là de l'objectif le plus mal défini, car trop souvent abordé par la seule question du prix de l'énergie, alors que c'est désormais la capacité à innover qui fonde la compétitivité des entreprises européennes dans la mondialisation ${ }^{3}$.

\footnotetext{
${ }^{1}$ Article rédigé en octobre 2016, NdE.

${ }^{2}$ Sami Andoura, Jean-Arnold Vinois, De la Communauté européenne de l'énergie à l'Union de l'énergie une proposition politique pour le court et le long terme [en ligne], préface de Jacques Delors, Institut Jacques Delors, Paris, janvier 2015 (Études \& Rapports ; 107). Disponible sur : http://www.delorsinstitute.eu/media/ unionenergie-andouravinois-ijd-jan15.pdf?pdf=ok [consulté le 27/04/2017].

${ }^{3}$ Thomas Pellerin-Carlin, Pierre Serkine, From Distraction to Action - for a Bold European Energy Union Innovation Strategy [en ligne], Institut Jacques Delors, Paris, 14 juin 2016 (Policy Paper ; 167). Disponible sur: http://www.institutdelors.eu/media/energyunioninnovationstrategy-pellerincarlinserkine-jdi-june16.pdf? pdf=ok [consulté le 27/04/2017].
} 
Dans ce contexte, la communication sur l'Union de l'énergie promulguée par la Commission européenne le 25 février $2015^{4}$ est d'une grande clarté sur la vision qui doit animer l'Union de l'énergie : "une Union de l'énergie focalisée sur le citoyen [...] nous devons abandonner le modèle économique reposant sur les combustibles fossiles [...] donner du [pouvoir] aux consommateurs [...] [créer une] coordination des politiques nationales ». Dès lors, il convient de regarder les politiques énergétiques nationales à l'aune de leurs convergences et divergences avec le projet d'Union de l'énergie. Dans les cas du Royaume-Uni, de la Suède et de la Pologne, il convient de s'interroger sur deux éléments :

- la situation énergétique actuelle : quels points communs et différences entre une situation nationale et celle des autres États européens/de l'Union européenne?

- les objectifs nationaux de politique énergétique : sur quels éléments voit-on une convergence, une complémentarité ou une opposition entre les objectifs d'un État européen, ceux d'autres États européens et ceux de l'Union européenne?

\footnotetext{
${ }^{4}$ Commission européenne, Paquet «Union de l'énergie »-Communication de la Commission au Parlement européen, au Conseil, au Comité économique et social européen, au Comité des régions et à la Banque européenne d'investissement - Cadre stratégique pour une Union de l'énergie résiliente, dotée d'une politique clairvoyante en matière de changement climatique [en ligne], Bruxelles, 25 février 2015 [COM(2015) 80 final]. Disponible sur: http://eur-lex.europa.eu/resource.html?uri=cellar:1bd46c90-bdd4-11e4-bbe1-01aa75ed 71a1.0003.03/DOC_1\&format=PDF [consulté le 27/04/2017].
} 


\section{La politique énergétique du Royaume-Uni : orientations passées et futures ${ }^{1}$}

\section{Joseph DUtTon}

La politique énergétique est au cœur du projet européen depuis sa naissance, et pas seulement dans l'approche réglementaire large des marchés et de la normalisation sur laquelle la Commission européenne légifère de nos jours. L'énergie était initialement considérée sous l'angle restrictif de la sécurité de l'approvisionnement et de la mise en commun des ressources. Le traité établissant la Communauté européenne du charbon et de l'acier (CECA) a été signé par la Belgique, l'Allemagne, la France, l'Italie, le Luxembourg et les Pays-Bas en avril 1951, et est entré en vigueur en juillet 1952. Il a été suivi par la création des marchés communs du charbon, du minerai de fer, du métal et de l'acier en 1953, puis par celle de la Communauté européenne de l'énergie atomique (Euratom) en 1957.

Graphique 1 : Dépendance européenne envers les importations d'énergie, 1998-2014 (en \%)

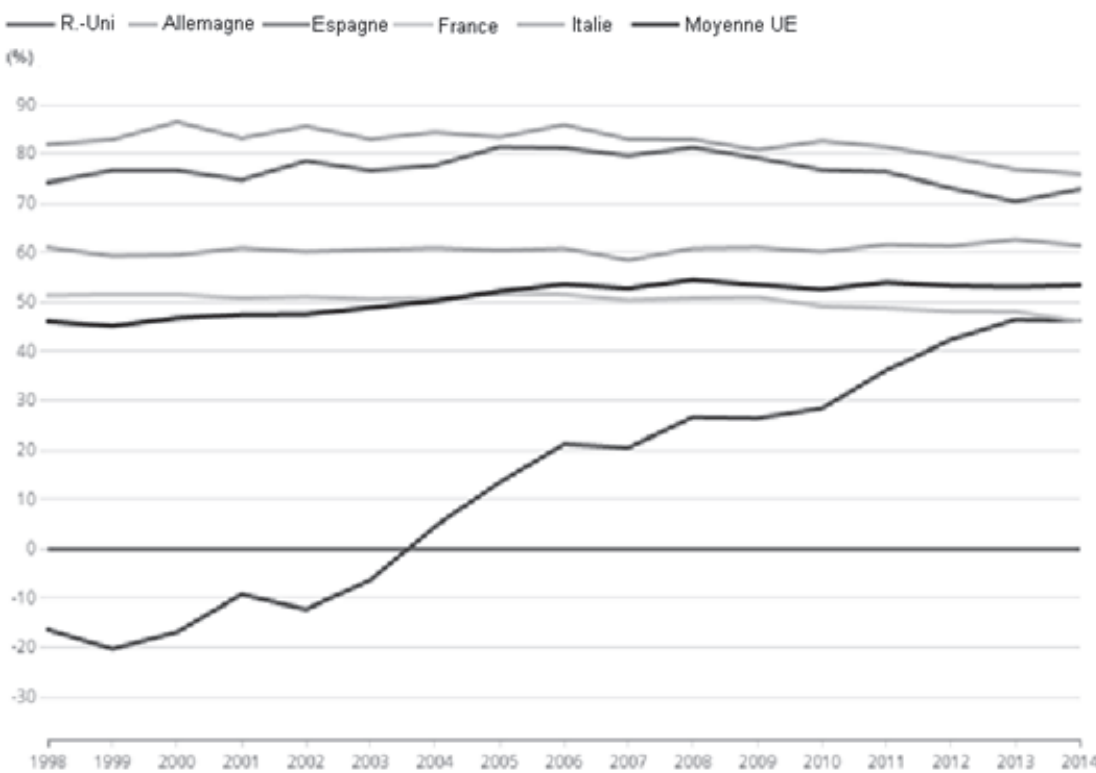

Source : Office for National Statistics².

\footnotetext{
${ }^{1}$ Article rédigé en novembre 2016, NdE.

${ }^{2}$ Office for National Statistics (ONS), UK Energy: How Much, What Type and Where From? [en ligne], Newport, 15 août 2016. Disponible sur : http://visual.ons.gov.uk/uk-energy-how-much-what-type-and-wherefrom/ [consulté le 02/03/2017].
} 
Pendant une grande partie de cette période, le Royaume-Uni était autosuffisant en matière énergétique. Le développement de l'exploitation pétrolière et gazière en mer du Nord à partir des années 1960 a pris la relève d'une vaste industrie charbonnière - tout en provoquant, en fin de compte, son effondrement. Depuis qu'il a rejoint l'Union européenne (UE) alors dénommée Communauté économique européenne (CEE) - en 1973, le Royaume-Uni participe activement à une approche plus régionale et coopérative de l'énergie. C'est particulièrement notable depuis 20 ans, puisque la chute de la production indigène de combustibles fossiles a forcé le Royaume-Uni à s'orienter vers une approche plus internationale et européenne - de la sécurité énergétique et de sa dépendance aux importations.

En observant l'ampleur de la demande énergétique du Royaume-Uni et le déclin de sa production nationale d'énergie, on peut définir la nature et le niveau de ses interactions avec l'UE sur les questions énergétiques. Le Royaume-Uni a été le moteur de certaines politiques, à la pointe de questions telles que la libéralisation du marché, le développement des énergies renouvelables et la lutte contre le réchauffement climatique. La politique énergétique du pays est largement définie par un trilemme énergétique: sécurité de l'approvisionnement, prix abordable, et qualité environnementale et lutte contre les changements climatiques. Elle réagit typiquement à l'évolution de l'offre et de la demande nationale, mais aussi au contexte politique et au gouvernement en place.

\section{Approvisionnement énergétique}

Alors qu'une grande partie de l'UE est dépendante des importations d'énergie, le Royaume-Uni a bénéficié d'une autosuffisance pendant presque tout le $\mathrm{XX}^{\mathrm{e}}$ siècle grâce à son industrie des énergies fossiles. La découverte de gaz naturel en mer du Nord dans les années 1960, suivie par la conversion au gaz du secteur de la production d'électricité dans les années 1990 - la «ruée vers le gaz » - a profondément changé le paysage de la production électrique. Le Royaume-Uni est devenu exportateur net d'énergie en 1981 grâce à la production croissante de la mer du Nord, et l'est resté jusqu'au milieu des années 2000. Plus récemment, la réduction de la consommation de charbon dans le secteur de l'électricité, associée à la croissance des énergies renouvelables, a encore modifié le mix énergétique du pays en posant de nouveaux défis en termes de sécurité d'approvisionnement.

\section{Pétrole et gaz de mer du Nord}

Au début des années 1970, le Royaume-Uni importait plus de 50 \% de sa consommation en énergie. Cette dépendance avait des conséquences financières considérables pour le pays, mais l'embargo pétrolier arabe de 1973 a également fait planer la menace d'une pénurie et mis en évidence les faiblesses en matière de sécurité énergétique. Les membres arabes de l'Organisation des pays exportateurs de pétrole (OPEP) ont appliqué un embargo sur les exportations de pétrole vers les États-Unis et plusieurs pays européens perçus comme leurs alliés, notamment le Royaume-Uni, l'Allemagne de l'Ouest et les Pays-Bas, à cause de leur soutien 
aux opérations militaires israéliennes contre des pays arabes. Mais auparavant, la crise de Suez en 1956-1957 et la découverte du champ gazier de Groningen aux Pays-Bas en 1959 avaient déjà incité le Royaume-Uni à exploiter ses propres ressources - cette année-là, plusieurs sociétés ont demandé des droits d'exploration dans les eaux britanniques, et la prospection sismique a commencé en $1962^{3}$. Le gouvernement a délivré ses premiers permis d'exploration pétrolière en mer du Nord en 1964, et le premier puits a été foré en 1967. La production de pétrole a démarré en 1975, avec une augmentation spectaculaire alors que de nombreux gisements entraient en production dans un court laps de temps. Les prix élevés du pétrole au niveau international à cause de l'embargo arabe rendaient également la production de la mer du Nord rentable malgré les importants coûts de développement dans cette région ${ }^{4}$. On a découvert du gaz en mer du Nord pour la première fois en 1964, mais c'est seulement l'année suivante que la première découverte commercialement viable a été effectuée par BP dans le gisement de West Sole. Ensuite, en 1966, Esso et Shell ont découvert du gaz sur le Leman Bank. La production est devenue significative à partir de 1968, et les rendements ont augmenté de 30 \% par an jusqu'en 1980 avant de culminer en 1999. En conséquence, la dépendance envers les importations est tombée de 52,5\% en 1974 à $26 \%$ en 1977. En 1981, le Royaume-Uni était devenu exportateur net d'énergie et l'est resté jusqu'en $2004^{5}$.

\section{Graphique 2 : Production de gaz naturel au Royaume-Uni, 1960-2015 (en GWh)}

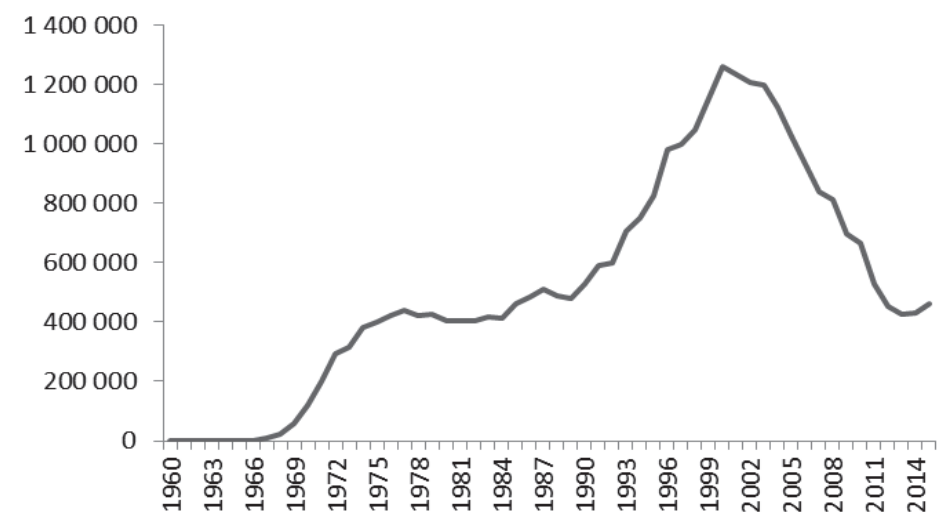

Source : ministère britannique des Entreprises, de l'Énergie et de la Stratégie industrielle (Department for Business, Energy and Industrial Strategy, BEIS ${ }^{6}$ ).

\footnotetext{
${ }^{3}$ Paul Cleary, Trillion Dollar Baby, Biteback, Londres, 2016, p. 12.

${ }^{4}$ Guy Lodge, «Thatcher and North Sea Oil - a Failure to Invest in Britain's Future », New Statesman, 15 avril 2013, p. [n.c.].

${ }^{5}$ Office for National Statistics (ONS), UK Energy: How Much, What Type and Where From? [en ligne], Newport, 15 août 2016. Disponible sur : http://visual.ons.gov.uk/uk-energy-how-much-what-type-and-wherefrom/ [consulté le 02/03/2017].

${ }^{6}$ BEIS, Historical Gas Data: Gas Production and Consumption and Fuel Input 1920 to 2015, Londres, 2016.
} 


\section{La « ruée vers le gaz »}

L'abondance de gaz naturel a incité le gouvernement à convertir au gaz naturel (contenant du méthane) les réseaux locaux de distribution de gaz du pays qui fonctionnaient au gaz de ville (gaz manufacturé), à développer le réseau national de transport dans les années 1970 pour le raccorder à ces réseaux locaux, et à construire une série de terminaux destinés à recevoir le gaz acheminé par des pipelines depuis les gisements offshore.

Par la suite, le gaz a gardé un rôle important dans le secteur du chauffage domestique, mais sa croissance dans le secteur de la production d'électricité baptisée la « ruée vers le gaz » - est principalement due à la politique gouvernementale, même si l'abondance de gaz indigène a également joué un rôle. Le résultat : la présence du gaz dans le secteur de l'électricité a explosé. En 1990, il n'existait aucune grande centrale électrique à gaz, mais en 1995 le gaz représentait $19 \%$ de la production d'électricité, puis $32 \%$ en $1998^{7}$. La première centrale à gaz à cycle combiné (combined cycle gas turbine, CCGT) a été commandée en 1992 ; en 1999, le réseau totalisait 16 GW de capacité, soit environ $23 \%$ de la capacité totale de production connectée ${ }^{8}$.

Avant la libéralisation du marché, à la fin des années 1980, le gouvernement donnait un accès préférentiel au réseau à l'énergie nucléaire et aux centrales à charbon. L'Office central de production d'électricité (Central Electricity Generating Board, CEGB) avait mis en place une série de contrats - dont certains sont restés en vigueur jusqu'en 1998 - entre les sociétés publiques British Coal et National Power pour un approvisionnement en charbon au-dessus des prix pratiqués sur les marchés internationaux ${ }^{9}$.

Mais l'explosion est également due en partie à la politique de la Communauté économique européenne (CEE) sur la protection de l'environnement. En 1988, celle-ci a mis en place la directive sur les grandes installations de combustion (88/609/CEE) visant à fermer progressivement les unités industrielles très polluantes et à réduire les émissions. Dans cette législation, le RoyaumeUni s'est vu assigner un objectif inférieur à celui de la plupart des autres pays, car l'importance des centrales à charbon dans son système électrique aurait impliqué des coûts plus élevés en comparaison ${ }^{10}$. Pour se mettre en conformité avec la directive, il aurait fallu moderniser $12 \mathrm{GW}$ de capacité. Cependant, en 1990, la Commission européenne a mis fin à l'interdiction d'utiliser du gaz pour produire de l'électricité, ce qui a permis aux sociétés du marché récemment pri-

\footnotetext{
${ }^{7}$ Department for Energy and Climate Change (DECC) [ministère de l'Énergie et du Changement climatique], Digest of UK Energy Statistics - 60th Anniversary Edition, Londres, 2008, p. 40.

${ }^{8}$ DECC, Third Progress Report of the Promotion and Use of Energy from Renewable Sources for the United Kingdom, Londres, 2016.

${ }^{9}$ Office of Gas and Electricity Markets (Ofgem), Review of Energy Sources for Power Stations [en ligne], Londres, 1998, p. 13. Disponible sur: https://www.ofgem.gov.uk/ofgem-publications/79084/review-energysources-power-stations-submission-dges-30-04.pdf [consulté le 02/03/2017].

${ }^{10}$ Dominique Finon., John Surrey, « The Future of EU Energy Policy », in Francis McGowan (dir.), European Energy Policies in a Changing Environment, Physica-Verlag, Heidelberg, 1996, p. 173.
} 
vatisé de construire des centrales au gaz approvisionnées par les stocks abondants de la mer du Nord, plutôt que de moderniser les anciennes centrales au charbon à un coût supérieur ${ }^{11}$. L'abondance et le faible prix du gaz ont été cruciaux pour le développement de nouvelles centrales CCGT à haut rendement, mais on a également affirmé que l'intervention du gouvernement, la recherche militaire et industrielle ainsi que le financement de la R\&D avaient aussi eu une influence $^{12}$.

\section{Graphique 3 : Production d'électricité par source, 1990-2015 (en TWh)}

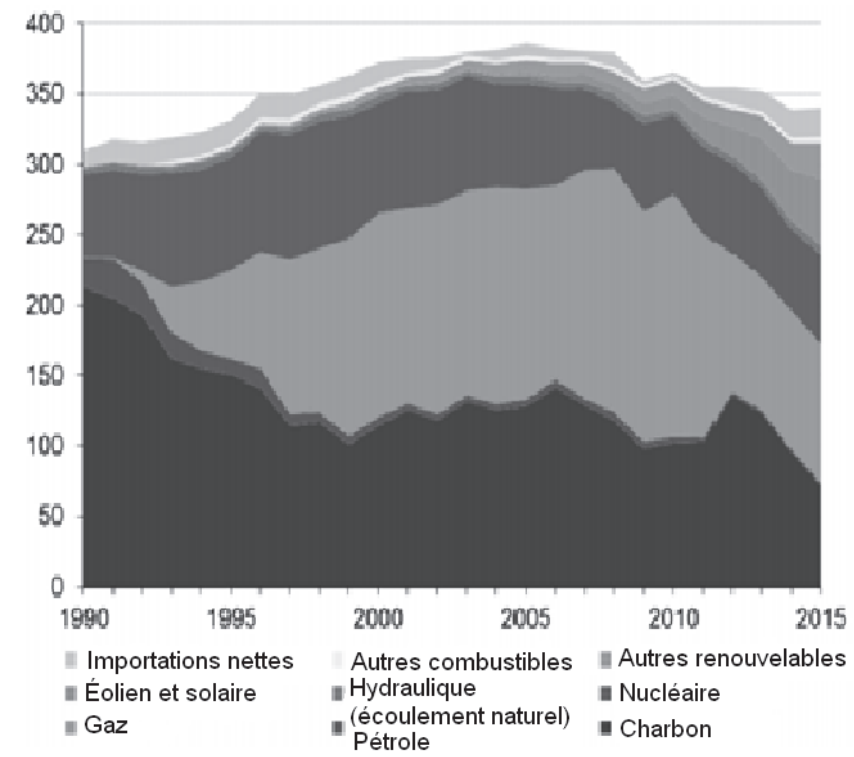

Source : BEIS ${ }^{13}$.

\section{Énergies renouvelables}

Tout comme le développement des réserves nationales de pétrole et de gaz, le développement des sources d'électricité renouvelables, en particulier l'éolien, est devenu un problème stratégique dans les années 1970 à cause de l'embargo arabe sur le pétrole. Dans le cadre de la privatisation et de la restructuration du marché britannique de l'énergie, la loi de 1989 sur l'électricité (Electricity Act) incluait des dispositions exigeant des sociétés régionales d'électricité qu'elles s'engagent sur une certaine capacité de production d'électricité à partir de

\footnotetext{
${ }^{11}$ Ibid.

12 Jim Watson, «The Technology that Drove the Dash for Gas », Power Engineering Journal, février 1997, p. [n.c.].

13 BEIS, Coal Generation in Great Britain [en ligne], Londres, 9 novembre 2016. Disponible sur : https://www.gov.uk/government/uploads/system/uploads/attachment_data/file/566929/Unabated_coal_closure _consultation_FINAL_v6.0_.pdf [consulté le 02/03/2017].
} 
sources d'énergie renouvelable. La première ferme éolienne terrestre du Royaume-Uni a été construite en 1990, tandis que la première ferme éolienne offshore n'a pas été bâtie avant 2001. La technologie éolienne était envisagée depuis les années 1980, mais on considérait que son coût était prohibitif, et dans les années 1990, la faiblesse des prix du pétrole et la ruée vers le gaz ont calmé les inquiétudes sur la sécurité de l'approvisionnement ${ }^{14}$.

\section{Graphique 4 : Production d'électricité à partir de sources d'énergie renouvelable, 2000-2014 (en \%)}

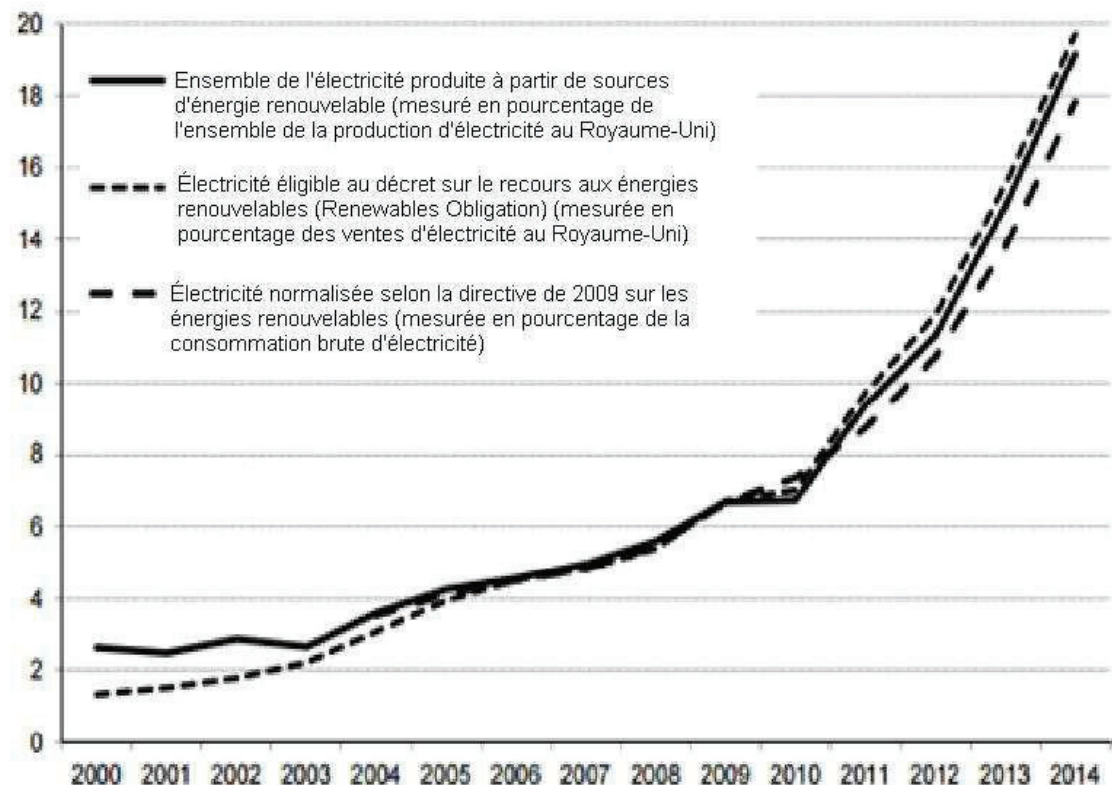

Source : ministère britannique de l'Énergie et du Changement climatique (Department for Energy and Climate Change, $\mathrm{DECC}^{15}$ ).

Par rapport aux autres membres de l'UE, le développement des énergies renouvelables au Royaume-Uni a été entravé par la disponibilité des combustibles fossiles indigènes, en particulier le charbon et le gaz de la mer du Nord ${ }^{16}$. Cependant, on a ensuite observé une forte croissance du secteur éolien britannique, en partie à cause de l'alignement des intérêts économiques et environnementaux sous-jacents chez les acteurs institutionnels et les promoteurs ${ }^{17}$. Cela a coïncidé avec une augmentation des importations d'énergie du Royaume-Uni. La part globale des éner-

\footnotetext{
${ }^{14}$ Jim Watson, «The Technology that Drove the Dash for Gas », Power Engineering Journal, février 1997, p. [n.c.].

${ }_{15}^{15}$ DECC, « Renewable Sources of Energy », Digest of UK Energy Statistics, Londres, 2015, p. [n.c.].

${ }^{16}$ Danyel Reiche, Mischa Bechberger, «Policy Differences in the Promotion of Renewable Energies in the EU Member States », Energy Policy, vol. 32, n 7, 2004, p. 843-849.

${ }^{17}$ Florian Kern, Adrian Smith, Chris Shaw, Rob Raven, Bram Verhees, « From Laggard to Leader: Explaining Offshore Wind Developments in the UK », Energy Policy, vol. 69, 2014, p. 635-646.
} 
gies renouvelables dans le mix électrique est restée faible jusqu'aux années 2000, où le développement des énergies renouvelables s'est taillé une place substantielle dans le champ des politiques de l'UE. Même si celle-ci avait précédemment cherché à encourager la croissance des énergies renouvelables par des mesures telles que la directive de 2001 sur la promotion de l'utilisation de l'énergie produite à partir de sources renouvelables (2001/77/CE), c'est la directive de 2009 sur les énergies renouvelables (2009/28/CE) qui a eu le plus d'effet.

Cette directive fixe un objectif européen de $20 \%$ de la consommation d'énergie provenant de sources renouvelables d'ici 2020. Quant au Royaume-Uni, il a adopté un objectif national de $15 \%$ d'ici 2020. Chaque État membre a fixé son propre objectif non contraignant et a défini son plan d'action national en matière d'énergies renouvelables dans lequel il est question des moyens à mettre en œuvre pour atteindre cet objectif. Le ministère britannique de l'Énergie et du Changement climatique a prévu à l'époque que pour atteindre l'objectif, il faudrait satisfaire $30 \%$ de la demande en électricité à partir d'énergies renouvelables d'ici 2020. La nouvelle directive s'appuyait sur celle de 2001, qui avait fixé un objectif communautaire de $22 \%$ d'ici 2010 , avec un objectif indicatif de $10 \%$ pour le Royaume-Uni.

Le Royaume-Uni a mis en place son programme de soutien des énergies renouvelables par un tarif de rachat (feed-in tariff, FiT) en avril 2010, quelques années après d'autres pays. Par exemple, l'Allemagne l'a mis en place en 1991, et le Danemark, l'Espagne, la Norvège et la Suède ont tous instauré leur propre régime avant 2000. Le FiT garantissait un prix fixe par unité d'électricité produite et injectée dans le réseau en fonction de la technologie renouvelable employée. Ce programme a rencontré un vif succès, avec 379531 installations enregistrées dans les trois premières années de fonctionnement. L'objectif initial du programme était d'atteindre 750000 installations enregistrées d'ici $2020^{18}$. En 2014, les énergies renouvelables satisfaisaient $7 \%$ de la demande britannique, contre seulement $1,3 \%$ en $2005^{19}$. Un rapport de la bibliothèque de la Chambre des communes affirme que l'accent mis par le Royaume-Uni sur les énergies renouvelables découle des objectifs et des politiques de l'UE ${ }^{20}$. L'impact de ces politiques sur la production d'électricité est visible dans la croissance des capacités provenant d'énergies renouvelables : de 9,2 GW en 2010, elles sont passées à 27,2 GW début $2015^{21}$. Les sources d'énergie renouvelable ont généré quelque $19,1 \%$ de l'électricité totale en 2015 , contre seulement $6,7 \%$ en $2009^{22}$.

\footnotetext{
${ }^{18}$ David Grover, The British Feed-in Tariff for Small Renewable Energy Systems: Can it Be Made Fairer? Rapport d'orientation du Centre for Climate Change Economics and Policy (CCCEP) et du Grantham Research Institute on Climate Change and the Environment, Leeds/Londres, octobre 2013.

${ }^{19}$ DECC, National Renewable Energy Action Plan for the United Kingdom, Londres, 2009.

${ }^{20}$ House of Commons Library, « Leaving the EU », Research Paper, ${ }^{\circ} 13 / 42,2013$.

${ }^{21}$ DECC, « Renewable Sources of Energy », Digest of UK Energy Statistics, 2015, p. [n.c.].

${ }^{22}$ Eurostat, Énergie - Statistiques illustrées [en ligne], Luxembourg, [s.d.]. Disponible sur : http://ec.europa. eu/eurostat/web/energy/statistics-illustrated [consulté le 02/03/2017].
} 


\section{Interconnexions électriques}

Les interconnexions sont de plus en plus importantes pour l'approvisionnement du Royaume-Uni en électricité ; elles en représentaient un peu moins de $7 \%$ en $2015^{23}$. L'Interconnexion France-Angleterre (IFA) de $2 \mathrm{GW}$ qui relie le Royaume-Uni à la France a été mise en service en 1986, tandis que la plus récente, l'East-West Interconnector (EWIC), a été terminée en 2012. Un certain nombre d'interconnexions sont en cours de développement, non seulement pour ajouter des connexions avec des partenaires existants, mais aussi pour en établir de nouvelles avec de nouveaux marchés en Islande, en Norvège et au Danemark.

\section{Carte 1 : Production d'électricité à partir de sources d'énergie renouvelable, 2000-2014 (en \%)}

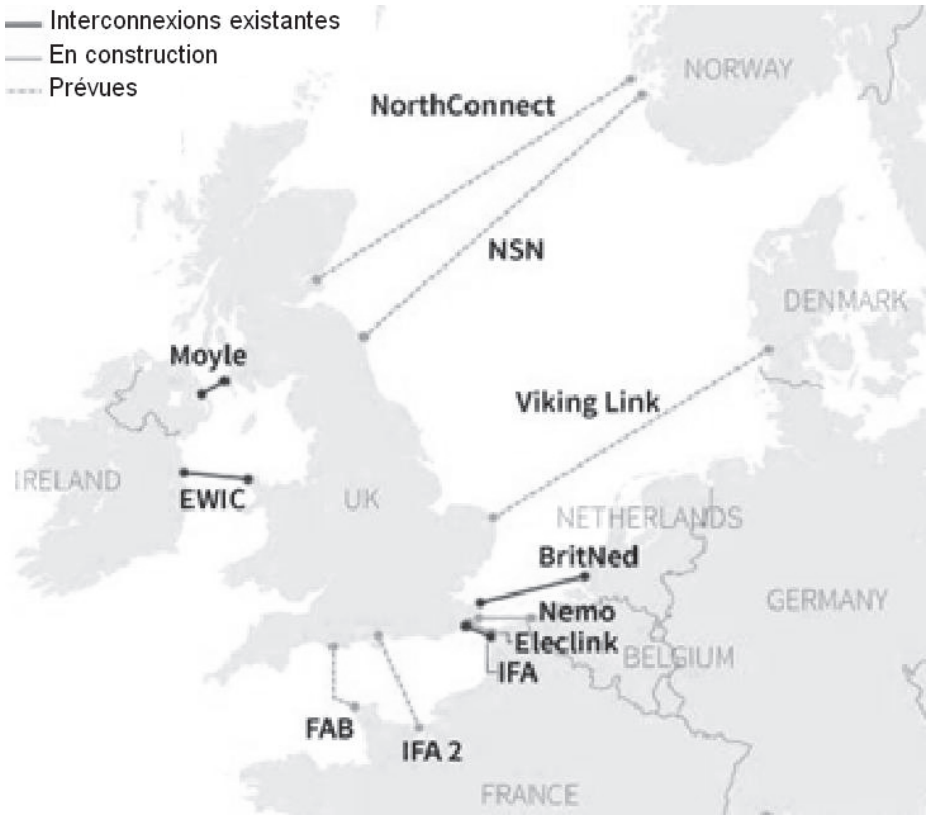

Source : Reuters ${ }^{24}$.

Les interconnexions font partie intégrante du marché européen de l'énergie en permettant des échanges transfrontaliers entre les États membres ; de fait, elles constituent le «hardware » sur lequel s'appuient la réglementation et la législation du marché unique, le «software $»^{25}$. La connexion à d'autres marchés permet au Royaume-Uni d'importer de l'électricité à moindre coût ; National Grid a estimé

\footnotetext{
${ }^{23}$ BEIS, Fuel Used in Electricity Generation and Electricity Supplied, Londres, 29 septembre 2016.

${ }^{24}$ Susanna Twidale, Britain Banks on Electricity Imports to Keep Lights on [en ligne], Reuters, Londres, 25 septembre 2015. Disponible sur: http://www.reuters.com/article/britain-power-interconnectors-idUSL5 N11V1LO20150925 [consulté le 02/03/2017].

${ }^{25}$ Joseph Dutton, «EU Energy Policy and the Third Package », EPG Working Paper, n¹505, 2015, p. [n.c.].
} 
que chaque gigawatt additionnel de capacités d'interconnexion entre le RoyaumeUni et l'Europe pourrait faire baisser le prix de gros de 1 à $2 \%$, soit l'équivalent de 3 millions de livres sterling d'économies par jour d'ici $2020^{26}$. La Commission a également noté que le coût de gros de l'électricité dans l'UE avait baissé d'un tiers entre 2008 et 2012, en partie du fait de l'intégration des marchés ${ }^{27}$.

La Commission encourage le développement de nouvelles interconnexions, qui sont primordiales pour créer un marché unique en permettant les échanges transfrontaliers d'électricité et le développement de marchés régionaux, ce qu'on appelle le «couplage des marchés ». C'est un élément central du développement et du fonctionnement du marché interne de l'énergie, de plus en plus présent sur les marchés de gros. En mai 2014, la Commission a proposé une augmentation des niveaux d'interconnexion européens pour atteindre $15 \%$ de la capacité de production installée dans chaque État membre d'ici 2030, alors que l'objectif précédent était de $10 \%$. L'année suivante, un rapport sur la progression vers cet objectif établissait que 14 des 28 États membres avaient toujours une capacité d'interconnexion inférieure ou égale à $10 \%$, y compris le Royaume-Uni (6\%) et des pays tels que l'Espagne (3\%) et l'Irlande (9\%) qui devraient devenir d'importants exportateurs d'énergie éolienne à l'avenir.

\section{Réchauffement climatique et politique environnementale}

Même si les combustibles fossiles ont toujours été le pilier du secteur de l'énergie britannique et de son économie au sens large, le pays a toujours fait preuve d'une volonté politique de s'attaquer au réchauffement climatique : les gouvernements successifs de Margaret Thatcher, Tony Blair, Gordon Brown et plus récemment la coalition libérale-conservatrice ont tous soutenu une réduction des émissions de carbone, que ce soit au plan national ou international ${ }^{28}$. Dernièrement, à l'occasion de l'accord conclu en 2014 sur le Cadre d'action de l'UE en matière de climat et d'énergie à l'horizon 2030, Ed Davey, secrétaire d'État britannique à l'Énergie et au Changement climatique, a fait remarquer combien le Royaume-Uni avait été à la pointe des débats sur le climat et milité pour un objectif ambitieux ${ }^{29}$, et avait été un participant prépondérant aux négociations de la COP21 sur le climat à Paris en 2015.

La croissance des énergies renouvelables et la décarbonation du secteur de la production d'électricité peuvent être imputées à la politique menée par le Royaume-Uni, comme sa loi de 2008 sur les changements climatiques (Climate

\footnotetext{
${ }^{26}$ National Grid, Getting More Connected - The Opportunity from Greater Electricity Interconnection, Warwick, 2014.

${ }^{27}$ Commission européenne, COM/2015/082 Final, Bruxelles, 2015.

${ }^{28}$ Dieter Helm, «Government Failure, Rent-Seeking, and Capture: the Design of Climate Change Policy », Oxford Review of Economic Policy, vol. 26, n 2, 2010, p. 182-196.

${ }^{29}$ DECC, Running Hours During Winter 2013-14 for Plants Opted-Out of the Large Combustion Plant Directive [en ligne], Londres, 2014. Disponible sur : https://www.gov.uk/government/uploads/system/uploads/ attachment_data/file/357531/LCPD.pdf [consulté le 02/03/2017].
} 
Change Act), mais la politique de l'UE a également joué un rôle. L'UE a introduit des règles sur la décarbonation dans un certain nombre de domaines, notamment le transport, le chauffage et l'efficacité énergétique. En ce qui concerne le secteur de la production d'électricité, les principales règles mises en œuvre sont la directive sur les grandes installations de combustion et celle sur les énergies renouvelables. Même si les aspects environnementaux de ces règles sont à saluer, au Royaume-Uni on a avancé l'argument qu'elles avaient eu un impact négatif sur l'industrie de la production d'électricité, à tel point que l'on pourrait craindre pour la sécurité de l'approvisionnement, les investissements dans de nouvelles centrales thermiques ayant été freinés ${ }^{30}$. Cependant, on pourrait également considérer que l'augmentation de la capacité de production indigène par l'investissement dans les énergies renouvelables et d'autres objectifs à faibles émissions de carbone a eu un effet largement positif sur la sécurité d'approvisionnement du Royaume-Uni ${ }^{31}$.

\section{Le Climate Change Act}

La loi britannique de 2008 sur le réchauffement climatique a été la première au monde à légiférer sur la réduction des émissions de carbone. Elle a fixé un objectif contraignant de réduction de $80 \%$ par rapport aux niveaux de 1990 d'ici $2050^{32}$. Cette loi précède la réglementation européenne sur la maîtrise des émissions de dioxyde de carbone - contrairement à celle qui s'attaque à la pollution mise en place dans le cadre du troisième paquet énergétique en 2009. La loi adoptée par le parti travailliste a été saluée par le monde politique, l'industrie et les militants environnementaux, et a obtenu un large soutien de tous les partis au Parlement. En effet, cette loi était conçue comme un moyen de contraindre les gouvernements ultérieurs à s'attaquer au réchauffement climatique quels que soient les changements de gouvernement, et fournissait un cadre stable dans lequel développer des technologies et des infrastructures à faibles émissions de carbone $^{33}$. En termes institutionnels pour le Royaume-Uni, il est à noter que cette loi créait le ministère de l'Énergie et du Changement climatique (DECC), qui a renforcé la présence du réchauffement climatique dans le processus législatif, mais également assuré une responsabilité pour la définition d'objectifs et l'affectation de politiques ${ }^{34}$. Le DECC a repris au ministère des Affaires économiques, des Entreprises et de la Réforme réglementaire les compétences relatives

\footnotetext{
${ }^{30}$ Christian Egenhofer, Arno Behrens, Richard S.J. Tol, Michel Bethélemy, François Lévêque, Jaap C. Jansen, « Does Europe Need a Comprehensive Energy Policy? », Intereconomics, vol. 46, n 3, 2011, p. 124-142.

${ }^{31}$ Michael Grubb, Lucy Butler, Paul Twomey, « Diversity and Security in UK Electricity Generation: The Influence of Low-Carbon Objectives », Energy Policy, vol. 34, n ${ }^{\circ}$ 8, 2006, p. 4050-4062.

${ }^{32}$ HM Government [Gouvernement de Sa Majesté], Climate Change Act 2008 [en ligne], Londres, 2008. Disponible sur: http://www.legislation.gov.uk/ukpga/2008/27/part/1/crossheading/the-target-for-2050 [consulté le $02 / 03 / 2017]$.

${ }^{33}$ Matthew Lockwood, «The Political Sustainability of Climate Policy: The Case of the UK Climate Change Act », Energy Policy, vol. 23, n 5, 2013, p. 1339-1348.

${ }^{34}$ Ibid.
} 
à l'énergie, et au ministère de l'Environnement, de l'Alimentation et des Affaires rurales celles qui relèvent du réchauffement climatique.

\section{Sortie progressive du charbon}

En 2016, le gouvernement a annoncé une proposition visant à restreindre l'utilisation des centrales à charbon sans dispositif de captage et stockage du carbone d'ici 2023, pour une sortie totale d'ici $2025^{35}$. Cette proposition a été saluée, mais la sortie du charbon est en fait en cours depuis de nombreuses années : ce processus trouve ses racines réglementaires à la fin des années 1980 et au début des années 1990, comme on l'a détaillé plus haut, lorsque la Communauté économique européenne a cherché à réduire les émissions de carbone. Les politiques nationales ont également fait diminuer la production d'électricité par des centrales à charbon : par exemple, la suppression de l'accès préférentiel au réseau pour les centrales au charbon a lancé la ruée vers le gaz. La production d'électricité par des centrales à charbon a fortement chuté tout au long des années 1990 à cause du secteur gazier émergent, passant d'un total de 184,7 TWh en 1989/90 à 95,7 TWh en 1997, soit une chute de 48 \%. Sur la même période, les capacités de production au charbon dans le réseau ont baissé de seulement $26 \%$, ce qui montre que c'est l'utilisation qui a diminué, avec des centrales restées inactives dans le système. La part du charbon dans la production totale d'électricité est tombée de $72 \%$ en $1989 / 90$ à $34 \%$ en $1997^{36}$. En 2009, le gouvernement travailliste a modifié la législation sur la planification pour imposer un système de captage et stockage du carbone (carbon capture and storage, $\mathrm{CCS}$ ) à toute nouvelle centrale électrique à charbon, tandis que les autres centrales thermiques (à gaz ou à biomasse) d'une capacité de production supérieure à $300 \mathrm{MW}$ doivent être « compatibles CCS » afin de permettre l'ajout de cette technologie ultérieurement ${ }^{37}$. Dans les faits, cette mesure a stoppé la construction de nouvelles centrales à charbon.

Au niveau de la politique européenne, la directive de 2001 sur les grandes installations de combustion (GIC) visait à mettre fin au fonctionnement des centrales industrielles ayant une puissance thermique nominale supérieure ou égale à $50 \mathrm{MW}$ - mises en service avant 1987 - afin de réduire les émissions de dioxyde de soufre, d'oxyde d'azote et de particules. À l'époque, cette directive s'appliquait à toutes les centrales à charbon britanniques et leur donnait trois possibilités : respecter les limites d'émissions spécifiées dans la directive, s’inscrire à un

\footnotetext{
35 Amber Rudd, Speech on a New Direction for UK Energy Policy [en ligne], Government Digital Service, Londres, 18 novembre 2015. Disponible sur : https://www.gov.uk/government/speeches/amber-rudds-speechon-a-new-direction-for-uk-energy-policy [consulté le 02/03/2017].

${ }^{36}$ Office of Gas and Electricity Markets (Ofgem), Review of Energy Sources for Power Stations [en ligne], Londres, 1998. Disponible sur : https://www.ofgem.gov.uk/ofgem-publications/79084/review-energy-sourcespower-stations-submission-dges-30-04.pdf [consulté le 02/03/2017].

${ }^{37}$ Practical Law, Consent Given for New Gas and Coal Power Stations with CCS Capability [en ligne], Londres, 2009. Disponible sur : http://uk.practicallaw.com/5-385-0123?q=CCS [consulté le 02/03/2017].
} 
plan national de réduction des émissions dont les participants respecteraient des objectifs collectifs de réduction et échangeraient des quotas d'émission, ou bien fonctionner pendant un maximum de 20000 heures entre le $1^{\mathrm{er}}$ janvier 2008 et le 31 décembre 2015, l'option dite de «retrait ${ }^{38}$. La directive a été remplacée et renforcée par la directive relative aux émissions industrielles (2010/75/UE), entrée en vigueur le $1^{\mathrm{er}}$ janvier 2016. Celle-ci étend le champ et la profondeur de la réglementation en regroupant sept directives antérieures.

En application de la directive GIC, 6 des 17 centrales à charbon opérationnelles au Royaume-Uni ont opté pour le retrait, ce qui a supprimé $8 \mathrm{GW}$ de capacité sur les $28 \mathrm{MW}$ actifs en $2008^{39}$. Après l'entrée en vigueur de la directive sur les émissions industrielles, d'autres fermetures ont eu lieu. En décembre 2015, le Royaume-Uni comptait $17 \mathrm{GW}$ de capacité opérationnelle au charbon, dont environ 5 GW devaient fermer d'ici l'été 2016. Sur le restant, au moins $5 \mathrm{GW}$ sont conformes à la directive sur les émissions industrielles et demeureront opérationnels après 2025 ; quant au statut des sept derniers gigawatts, il reste à déterminer ${ }^{40}$. L'usage du charbon a continué à baisser, au point qu'en mai 2016, pendant une brève période il n'y a eu aucune production d'électricité sur le réseau à partir de charbon, et ce pour la première fois depuis 1882. En avril, l'énergie solaire a généré plus d'électricité que le charbon pour la première fois, et cette tendance s'est maintenue pendant le reste de l'année, avec une production solaire supérieure à celle du charbon entre avril et septembre ${ }^{41}$.

\section{Libéralisation du marché de l'énergie}

Une politique européenne des marchés a commencé à émerger dans les années 1990, mais c'est sous la présidence britannique de l'UE en 2005 que des progrès considérables ont été accomplis vers le marché unique de l'énergie. Pendant cette présidence, les priorités énoncées par le gouvernement en matière d'énergie incluaient la promotion de marchés de l'énergie compétitifs et ouverts, de la sécurité d'approvisionnement à long terme, et la lutte contre les changements climatiques ${ }^{42}$. Cependant, le processus avait démarré bien plus tôt au Royaume-Uni, avec la loi de privatisation du secteur électrique en 1989 (Electricity Act). Celle-ci a divisé la compagnie publique CEGB en quatre sociétés, dont trois ont reçu des actifs de production, et la quatrième le réseau de distribution en Angleterre et au Pays de Galles. La méthode britannique de libéralisation a même servi de modèle dans l'UE pour son marché unique de l'éner-

\footnotetext{
${ }^{38}$ Rob Gross, Jamie Speirs, Adam Hawkes, Simon Skillings, Phil Heptonstall, Could Retaining Old Coal Lead to a Policy Own Goal?, Centre for Energy Policy and Technology, Imperial College London, Londres, 2014 (ICEPT Research Report)

${ }^{39}$ DECC, Running Hours During Winter 2013-14 for Plants Opted-Out of the Large Combustion Plant Directive [en ligne], Londres, 2014. Disponible sur : https://www.gov.uk/government/uploads/system/uploads/ attachment_data/file/357531/LCPD.pdf [consulté le 02/03/2017].

${ }^{40}$ Argus Media, «UK Pledges Coal Plant Closures », Argus European Electricity, 18 novembre 2015, p. [n.c.].

${ }^{41}$ Simon Evans, « Analysis: UK Solar Beats Coal Over Half a Year », Carbon Brief, 4 octobre 2016, p. [n.c.].

${ }^{42}$ Richard Whitman, Gareth Thomas, Two Cheers for the UK's EU Presidency, Chatham House, Londres, 2005.
} 
gie et pour les règles du troisième paquet énergie de $2009^{43}$. L'influence du Royaume-Uni dans ce domaine a été telle que celui-ci a non seulement déterminé la nature des règles de libéralisation du marché dans l'UE, mais qu'il a également eu plus d'impact sur la libéralisation du secteur de l'électricité en Norvège, membre de l'Espace économique européen (EEE), que les précédentes politiques européennes ${ }^{44}$.

Après la présidence britannique, la Commission a mené une enquête sur le secteur de l'énergie, qui a permis d'identifier un certain nombre de lacunes des marchés, notamment la concentration des participants sur les marchés nationaux, le manque de liquidité du marché et la trop faible intégration entre les marchés des États membres ${ }^{45}$. En conséquence, la Commission a élaboré un nouvel ensemble de règles, appelé le troisième paquet énergie, qui est entré en vigueur en 2011. Celui-ci consistait en deux directives et trois règlements, visant en priorité les domaines suivants : dissocier les fournisseurs d'énergie (y compris la production) des opérateurs du réseau, renforcer l'indépendance des régulateurs de l'énergie, créer l'Agence de coopération des régulateurs de l'énergie (Agency for the Cooperation of Energy Regulators, ACER), renforcer la coopération transfrontalière des gestionnaires de réseau de transport (via ENTSO $^{46}$-E pour l'électricité et ENTSO-G pour le gaz) et accroître la transparence sur les marchés de détail ${ }^{47}$.

Les directives antérieures sur les règles communes des marchés nationaux de l'électricité et du gaz ont été remplacées, et les nouveaux règlements ont instauré des règles dans des domaines tels que l'accès aux réseaux de transport du gaz naturel, les conditions d'accès aux réseaux afin de permettre les échanges transfrontaliers d'électricité, et la création de l'ACER. La dissociation (unbundling), dans le cadre du troisième paquet, marque un changement par rapport aux précédentes directives. La nouvelle directive a en effet introduit une «séparation structurelle» entre les gestionnaires de réseau de transport et les activités de génération, de production et de fourniture d'énergie, dont le but était d'éviter les conflits d'intérêts et d'assurer la transparence ${ }^{48}$. Le troisième paquet cherchait également à assurer l'indépendance des autorités de régulation vis-à-vis des gouvernements et des acteurs de l'industrie, tandis que le précé-

\footnotetext{
${ }^{43}$ Dieter Helm, « Government Failure, Rent-Seeking, and Capture: the Design of Climate Change Policy », Oxford Review of Economic Policy, vol. 26, n ${ }^{\circ}$ 2, 2010, p. 182-196.

${ }^{44}$ Ian Bartle, «Europeans Outside the EU: Telecommunications and Electricity Reform in Norway and Switzerland », Governance, vol. 19, n ${ }^{\circ}$ 3, 2006, p. 407-436.

${ }^{45}$ Commission européenne, DG Competition Report on Energy Sector Inquiry [en ligne], Bruxelles, 2007. Disponible sur : http://ec.europa.eu/competition/sectors/energy/2005_inquiry/full_report_part1.pdf [consulté le $02 / 03 / 2017]$.

${ }^{46}$ European Network of Transmission System Operators.

${ }^{47}$ Commission européenne, Market Legislation [en ligne], Bruxelles, [s.d.]. Disponible sur : http://ec.europa. eu/energy/node/50 [consulté le 02/03/2017].

${ }^{48}$ Commission européenne, Ownership Unbundling - The Commission's Practice in Assessing the Presence of a Conflict of Interest Including in Case of Financial Investors [en ligne], Bruxelles, 2013, p. 2. Disponible sur : http://ec.europa.eu/energy/sites/ener/files/documents/swd_2013_0177_en.pdf [consulté le 02/03/2017].
} 
dent paquet législatif, en 2003, ne prévoyait une harmonisation des règles et des pratiques que sur la base du volontariat ${ }^{49}$.

Les deux processus réglementaires jumelés de la décarbonation de la production d'électricité et de la libéralisation du marché sont au cœur de la politique énergétique de l'UE, mais il existe des tensions entre eux. Le RoyaumeUni en est un exemple notable, en particulier dans la manière dont la législation européenne favorisant les énergies renouvelables au détriment des autres formes de production d'électricité signifie, de fait, que le gouvernement intervient pour déterminer la composition du secteur de la production d'électricité, ce qui entre en contradiction avec sa position sur l'économie de marchés0'. Cette intervention de l'UE sur des bases normatives environnementales est également en contradiction avec ses propres règles essentielles de libéralisation du marché et de dissociation de l'industrie énergétique, puisque la promotion des énergies renouvelables et les programmes spécifiques de soutien mis en place par les États membres pour atteindre les objectifs fixés ont provoqué une distorsion des marchés ${ }^{51}$.

\section{5 et le Brexit : orientations politiques}

Après l'élection du gouvernement conservateur en 2015, les programmes d'incitation et les mécanismes de soutien aux énergies renouvelables instaurés après la directive sur les énergies renouvelables de 2009 ont été réduits. Le gouvernement a également mis en avant les nouvelles technologies gaz et nucléaire pour l'avenir de l'approvisionnement électrique et la décarbonation, en envisageant le développement de nouveaux gisements de gaz de schiste pour remplacer le charbon ${ }^{52}$. Même si le processus de sortie de l'UE n'a pas encore formellement commencé, le gouvernement britannique a déjà apporté des changements à sa politique énergétique. Le ministère de l'Énergie et du Changement climatique (DECC) a été démantelé après le référendum, et son successeur, le ministère des Entreprises, de l'Énergie et de la Stratégie industrielle (BEIS), a fait savoir que le gouvernement avait l'intention d'incorporer l'énergie au sein d'un ensemble plus large de politiques industrielles. Cependant, la suppression du réchauffement climatique - du moins dans le nom - et le manque de clarté en ce qui concerne l'avenir des règles européennes de qualité environnementale et de lutte contre le réchauffement climatique au Royaume-Uni font craindre aux groupes environnementaux que le gouvernement ne réduise le champ

\footnotetext{
${ }^{49}$ Per Ove Eikeland, «EU Internal Energy Market Policy », FNI Report, n 14, 2008, p. 12.

${ }^{50}$ Malcolm Keay, UK Electricity Market Reform and the EU, Oxford Institute for Energy Studies, Oxford, 2013.

${ }^{51}$ David Buchan, How to Create a Single European Electricity Market - and Subsidise Renewables, Centre for European Reform, Londres, avril 2012 (Centre for European Reform policy brief).

52 Amber Rudd, Speech on a New Direction for UK Energy Policy [en ligne], Government Digital Service, Londres, 18 novembre 2015. Disponible sur : https://www.gov.uk/government/speeches/amber-rudds-speechon-a-new-direction-for-uk-energy-policy [consulté le 02/03/2017].
} 
d'application et les exigences s'il instaure une nouvelle législation pour remplacer celle de l'UE.

Même avant le référendum, la politique énergétique du Royaume-Uni divergeait de celle de l'UE dans un certain nombre de domaines. Par exemple, l'incitation à développer le gaz de schiste met le pays en opposition avec d'autres États membres de l'UE - comme l'Allemagne, le Danemark, la France et l'Espagne - qui ont ou sont en train d'instaurer des interdictions ou des moratoires sur la fracturation hydraulique. Le nouveau gouvernement britannique a promis de revigorer le secteur balbutiant du gaz de schiste. L'UE n'a pas mis en place de réglementation globale à ce sujet. Jusqu'à présent, son implication s'est plutôt limitée à la réglementation environnementale - où elle joue déjà un rôle substantiel - et à des principes minimaux de sauvegarde dans son développement ${ }^{53}$. En août, le gouvernement a présenté des projets de distribution de recettes fiscales aux communautés locales touchées, et même directement aux ménages. Plus récemment, le gouvernement a cassé la décision de l'autorité de planification du conseil du comté du Lancashire et autorisé la recherche de gaz de schiste ${ }^{54}$. De la même manière que l'abondance de gaz naturel en mer du Nord a entraîné la « ruée vers le gaz » dans les années 1990 - et retardé la transition vers les énergies renouvelables -, le soutien actuel du gouvernement au développement du gaz de schiste repose largement sur le principe d'utilisation des réserves indigènes afin d'améliorer la sécurité de l'approvisionnement. De la même manière, après le résultat du référendum, la construction du nouveau réacteur nucléaire de Hinkley Point $\mathrm{C}$ a finalement été approuvée et signée par le gouvernement en septembre, après des années de tergiversations ${ }^{55}$.

\section{Après le référendum, avant la sortie}

Pendant la campagne du référendum, la secrétaire d'État à l'Énergie de l'époque, Amber Rudd, avait affirmé que le Brexit pourrait infliger un « choc de 500 millions de livres » au système énergétique, qui finirait par se répercuter sur la facture des consommateurs. Le rapport auquel Amber Rudd faisait référence, commandé par le gestionnaire de réseau de transport National Grid, étudiait un large éventail de risques auxquels le Brexit exposerait le secteur de l'énergie. $\mathrm{Ce}$ rapport concluait que les risques pour l'approvisionnement physique en énergie étaient faibles, mais que les surcoûts des investissements dans les infrastructures énergétiques constitueraient la menace la plus importante pour le

\footnotetext{
${ }^{53}$ Commission européenne, Environnement : la Commission européenne préconise des principes minimaux applicables au gaz de schiste [en ligne], Bruxelles, 22 janvier 2014. Disponible sur : http://europa.eu/rapid/ press-release_IP-14-55_fr.htm [consulté le 02/03/2017].

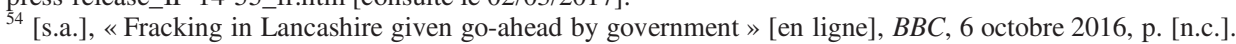
Disponible sur : http://www.bbc.co.uk/news/uk-england-lancashire-37567866 [consulté le 02/03/2017].

55 BEIS, « Hinkley Point C Contract Signed» [en ligne], Government Digital Service, Londres, 29 sep tembre 2016. Disponible sur : https:/www.gov.uk/government/news/hinkley-point-c-contract-signed [consulté le $02 / 03 / 2017]$.
} 
secteur de l'énergie après le Brexit. De même, toute période prolongée d'incertitude pourrait entraîner un report des investissements dans le secteur ${ }^{56}$. Les rapporteurs prévoyaient également un renchérissement des coûts à cause de la dévaluation de la livre sterling. Cela augmenterait le coût de l'énergie importée, ainsi que des équipements et services nécessaires pour moderniser les réseaux et l'infrastructure afin de les adapter à une part accrue d'énergies renouvelables, pour remplacer les centrales électriques à fermer, et pour développer un système énergétique décentralisé. Tout retard dans les investissements énergétiques vitaux pourrait encore réduire la sécurité de l'approvisionnement en énergie. Enfin, les auteurs du rapport suggéraient également que si le Royaume-Uni était exclu du marché unique de l'énergie, il pourrait perdre les avantages financiers offerts par l'intégration des marchés.

LA POLITIQUe ÉNERGÉTIQUe DU ROYAUME-Uni a été définie par la disponibilité de combustibles fossiles indigènes, comme le charbon, puis le pétrole et le gaz. Lorsque des menaces existentielles ont pesé sur la sécurité de l'approvisionnement, comme lors de l'embargo pétrolier arabe, les inquiétudes ont stimulé le développement de nouvelles sources d'énergie et de nouvelles technologies. Même si, dans les décennies suivantes, le réchauffement climatique et la sécurité environnementale ont occupé le devant de la scène, la sécurité de l'approvisionnement est restée le principal facteur déterminant de la politique énergétique - le développement des énergies renouvelables indigènes ayant été promu dans ce but. Cependant, le nouveau gouvernement devra prendre des décisions politiques sérieuses sur l'énergie dans les années à venir, d'ici la sortie de l'UE; pourtant, il est probable que les ressources seront consacrées au Brexit avant tout autre domaine. Parmi les défis à long terme, il faudra notamment adapter le système énergétique à la part croissante des sources d'électricité renouvelables intermittentes, garantir l'approvisionnement en électricité malgré la fermeture des centrales à charbon et à gaz vieillissantes, faire face au déclin des gisements de mer du Nord et à la fin de leur exploitation, et prendre des décisions sur les investissements dans de grandes infrastructures, notamment de nouvelles centrales nucléaires. Tous ces défis se présenteraient même sans le référendum, mais le gouvernement doit désormais affronter la perspective d'un éventuel détricotage et d'une réécriture de plusieurs décennies de législation, tout en veillant à ce que le système énergétique britannique soit prêt pour l'avenir.

\section{Traduction de Marie-Céline GEORG}

\footnotetext{
${ }^{56}$ Vivid Economics, The Impact of Brexit on the UK Energy Sector [en ligne], Londres, 2016. Disponible sur : http://www.vivideconomics.com/wp-content/uploads/2016/03/VE-note-on-impact-of-Brexit-on-theUK-energy-system.pdf [consulté le 02/03/2017].
} 


\title{
La politique énergétique d'autres pays d'Europe : le cas de la Suède'
}

\author{
Thomas B. JOHANSSON
}

La Suède fait face à d'importants défis en matière d'environnement, d'économie et de sécurité. Le dernier document de politique global pour la Suède est le projet de loi 2008/09:162 sur l'énergie et le climat, présenté par le gouvernement au Parlement. Ce texte présentait une vision de la Suède disposant d'un système énergétique durable et efficace, sans émissions nettes de gaz à effet de serre dans l'atmosphère, d'ici 2050.

Pour y parvenir, deux principaux instruments sont en place. D'une part, la participation au système européen d'échange de quotas d'émission (SEQE) couvrant les centrales électriques et les grandes industries, et d'autre part l'application d'une taxe $\mathrm{CO}_{2}$ depuis 1991, qui s'élève désormais à plus de 100 euros la tonne de $\mathrm{CO}_{2}$. La Suède a également pris une part active aux initiatives de l'Union européenne (UE) sur les questions climatiques et énergétiques.

Pour le secteur des transports, une commission a été créée en 2012 afin de clarifier le concept de "flotte de véhicules indépendante des combustibles fossiles ॥ introduit par la loi, et de proposer des mesures pour le mettre en œuvre. Cette commission est connue sous le sigle FFF2.

Récemment, la Suède a signé l'accord de Paris ${ }^{3}$ pour lutter contre le réchauffement climatique. Les signataires de cet accord se sont engagés à " conten[ir] l'élévation de la température moyenne de la planète nettement en dessous de $2^{\circ} \mathrm{C}$ par rapport aux niveaux préindustriels et [à poursuivre] l'action menée pour limiter la hausse de la température à $1,5^{\circ} \mathrm{C}$ par rapport aux niveaux préindustriels $"$. Ces limites sont proches, car le réchauffement a déjà dépassé $1^{\circ} \mathrm{C}$. Pour rester en dessous du plafond de $2^{\circ} \mathrm{C}$, les émissions mondiales de gaz à effet de serre devront être pratiquement ramenées à zéro d'ici 2050 , et pour limiter le réchauffement à $1,5^{\circ} \mathrm{C}$, il faudrait presque supprimer immédiatement toutes les émissions mondiales. Comme les deux tiers des émissions de gaz à effet de serre proviennent des combustibles fossiles, il faut réaliser d'importants efforts dans le secteur énergétique pour atteindre les objectifs de l'accord de Paris.

\section{Quelques objectifs nationaux}

Les émissions de gaz à effet de serre doivent être, d'ici 2020, de 40 \% inférieures au niveau de 1990 (pour les activités non couvertes par le SEQE).

La part des énergies renouvelables doit atteindre au moins $50 \%$ de la consommation d'énergie totale d'ici 2020.

\footnotetext{
${ }^{1}$ Article rédigé en janvier 2017, NdE.

${ }^{2}$ Fossiloberoende fordonsflotta: flotte de véhicules indépendante des combustibles fossiles. Le rapport de la commission a été présenté en décembre 2013 comme un rapport officiel du gouvernement, sous la référence SOU 2013:84.

${ }^{3}$ Accord de Paris, Nations unies, 2015.
} 
L'efficacité énergétique doit progresser, pour atteindre une réduction de $20 \%$ de l'intensité énergétique de l'économie d'ici 2020 par rapport à 2008.

\section{L'initiative « sans fossiles » de la Suède}

Cette initiative a été lancée en 2016 par le gouvernement. Son objectif est de faire de la Suède l'un des premiers pays sans combustibles fossiles du monde. La principale approche consiste à stimuler la coopération entre les secteurs public et privé, les universitaires, les ONG, etc. À ce jour, plus de 170 acteurs l'ont rejointe. Un coordinateur national a été nommé.

Si elle atteint son but, la Suède sera en conformité avec l'objectif de $2{ }^{\circ} \mathrm{C}$ de l'accord de Paris, sans plus.

\section{Énergie et émissions : rappel historique}

Le graphique 1 montre la croissance du produit intérieur brut (PIB), la consommation d'énergie primaire et la consommation d'électricité ainsi que les émissions de $\mathrm{CO}_{2}$ entre 1970 et 2015 . Les émissions ont baissé de $50 \%$ tandis que le PIB a augmenté de $150 \%$. La consommation d'énergie primaire est constante depuis les années 1980 et la consommation d'électricité a progressé jusqu'au milieu des années 1980 avant de se stabiliser, avec un léger déclin après l'an 2000.

Graphique 1 : Suède, 1970-2015 (année de référence : 1970)

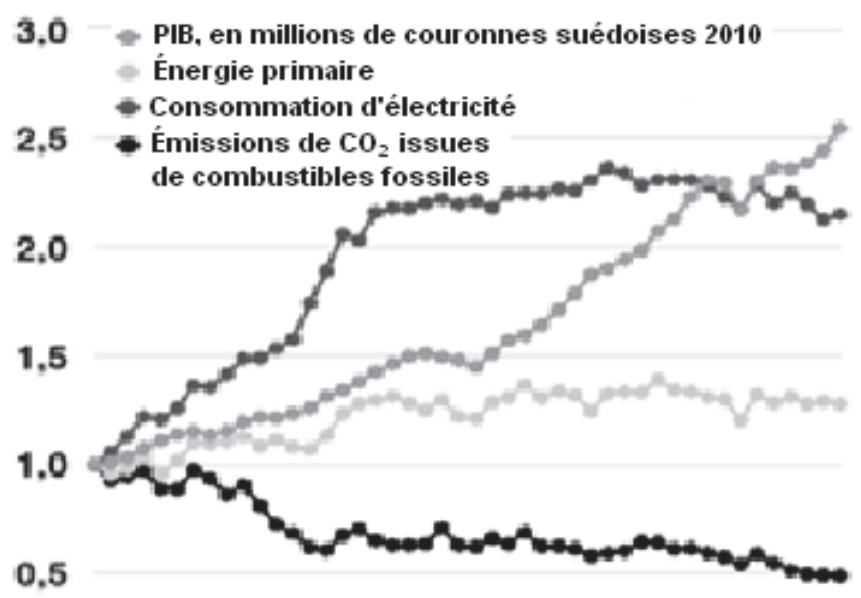

$1970197519801985199019952000 \quad 2005 \quad 2010 \quad 2015$

Source : Thomas B. Johansson, Tomas Kåberger, « Renewable Energy and Efficiency Development in Sweden », in Dörte Fouquet (dir.), ouvrage de la Fédération européenne des énergies renouvelables (EREF) à paraître. 
Les émissions présentées dans le graphique 1 sont mesurées sur une base géographique, ce qui signifie que les émissions recensées en Suède proviennent de tous les secteurs et de la production pour la consommation en Suède aussi bien que pour les exportations. Elles ne couvrent pas les émissions incluses dans les importations, les transports aériens internationaux et les transports maritimes.

Les émissions considérées du point de vue de la consommation sont présentées dans le schéma 1, tandis que le graphique 2 montre les données des deux points de vue, par habitant et de 1990 à 2012. Sur cette période, la population suédoise est passée de 8,6 à 9,6 millions d'habitants.

\section{Schéma 1 : Deux manières de considérer les émissions}

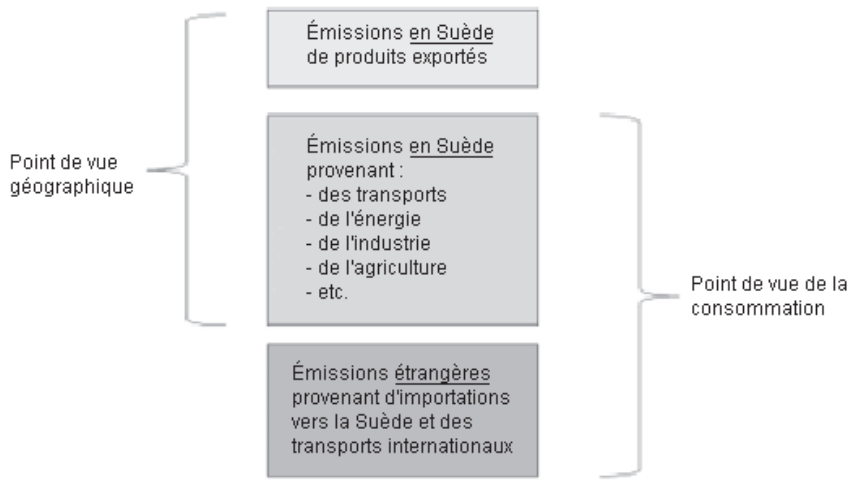

Source : Jörgen Larsson, Hållbara konsumtionsmönster - analyser av maten, flyget och den totala konsumtionens klimatpåverkan idag och 2050. En forskarantologi, Naturvårdsverket, Stockholm, 2015.

\section{Graphique 2 : Émissions de gaz à effet de serre :} le point de vue de la consommation vs le point de vue géographique

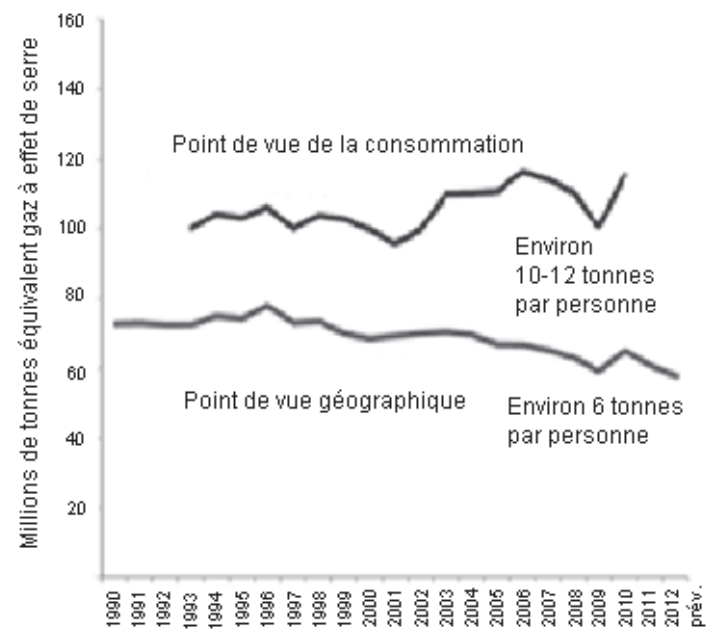

Source : Jörgen Larsson, 2015. 
Il est évident, au vu du graphique 2, que la production destinée à la consommation suédoise est associée à des émissions nettement supérieures au niveau enregistré en Suède. Le commerce est un facteur qui peut expliquer ce phénomène, les émissions des transports aériens internationaux en sont un autre, puisque ceux-ci ne sont pas inclus dans les statistiques géographiques.

Cela illustre l'importance d'inclure les niveaux et les modes de consommation dans les discussions sur l'empreinte écologique d'un pays. Jusqu'à présent, cela n'a pas été le cas dans les négociations internationales sur le climat.

Graphique 3 : Vols par personne et par année (1973-2013)

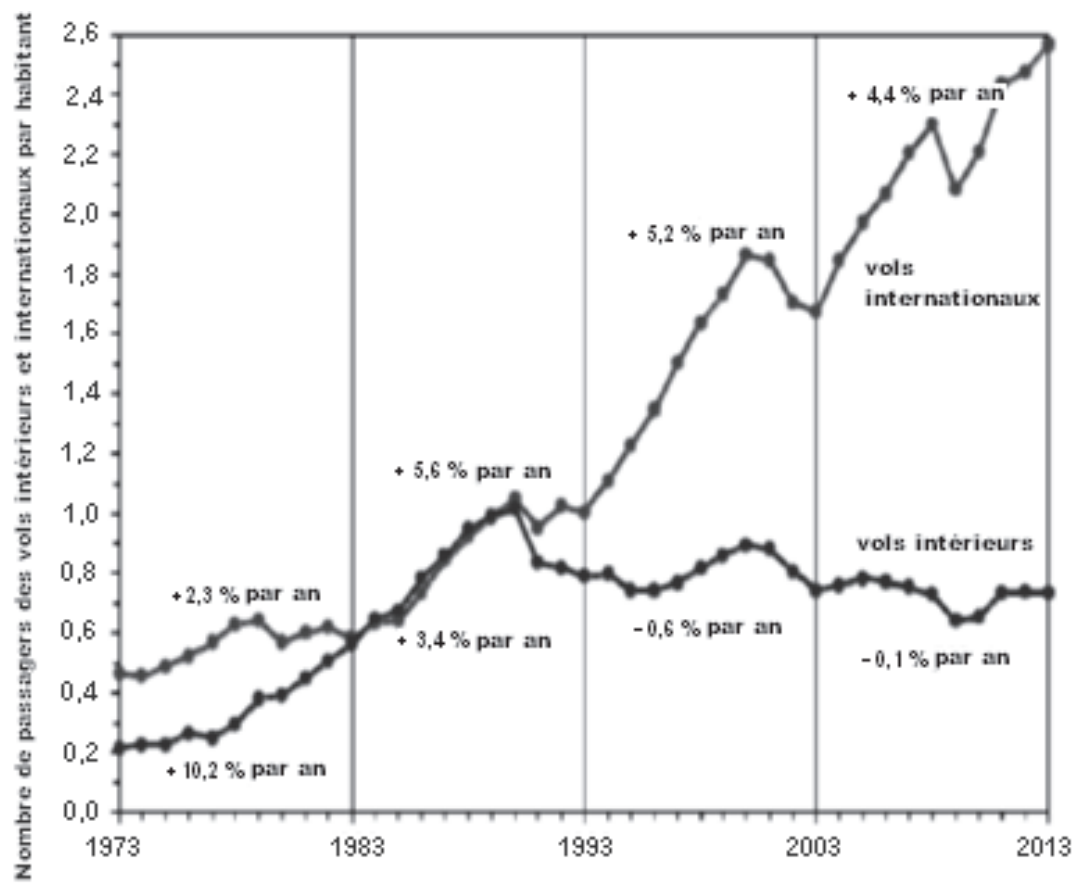

Source : Jörgen Larsson, Hållbara konsumtionsmönster - analyser av maten, flyget och den totala konsumtionens klimatpåverkan idag och 2050. En forskarantologi, Naturvårdsverket, Stockholm, 2015. Données : Trafikanalys, 2014.

Le graphique 3 montre la croissance du trafic aérien par habitant, pour les vols intérieurs et internationaux. Le trafic aérien intérieur diminue lentement depuis les années 1990, alors que le trafic aérien international par habitant continue à augmenter rapidement. Seule la consommation de carburant pour les déplacements intérieurs est incluse dans les statistiques d'émissions suédoises. Les émissions liées à l'utilisation de carburant pour le trafic aérien international sont donc un autre exemple d'émissions provenant de la consommation suédoise qui ne sont pas couvertes par les statistiques. Il faudra tenir compte de ces émissions par rapport aux ambitions mentionnées dans l'accord de Paris. 


\section{Graphique 4 : Approvisionnement en énergie selon les sources d'énergie (1970-2014, en TWh)}

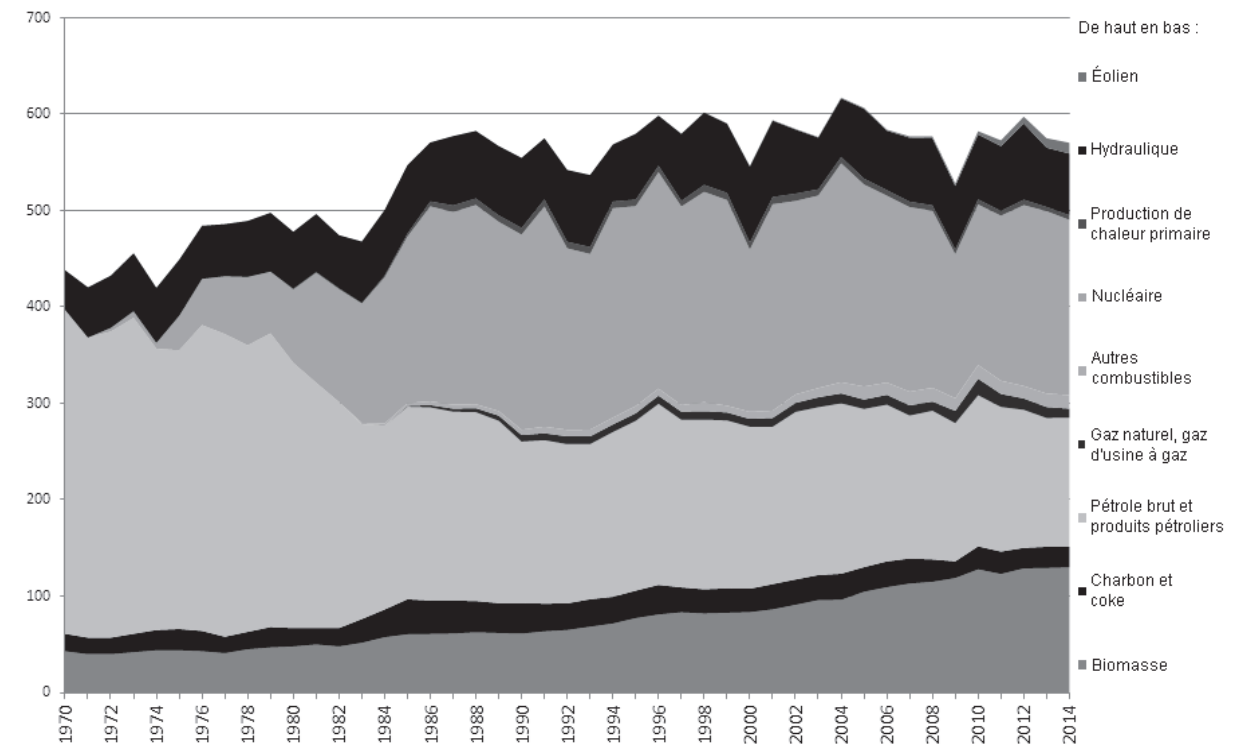

Source : Agence suédoise de l'énergie (Energimyndigheten), Energy in Sweden 2016.

Le graphique 4 présente l'approvisionnement en énergie. La réduction de l'utilisation du pétrole au début des années 1980 correspond à l'impact du passage au chauffage électrique direct dans les logements individuels à la place des chaudières au fioul. Cette consommation accrue d'électricité venait principalement de la capacité de production croissante d'énergie nucléaire. Pendant la période indiquée sur le graphique, la consommation de bioénergie a plus que doublé, principalement pour alimenter les centrales de chauffage urbain. Cette biomasse provenait principalement de déchets forestiers nationaux et de résidus.

\section{Graphique 5 : Part des énergies renouvelables (2005-2014, en \%)}

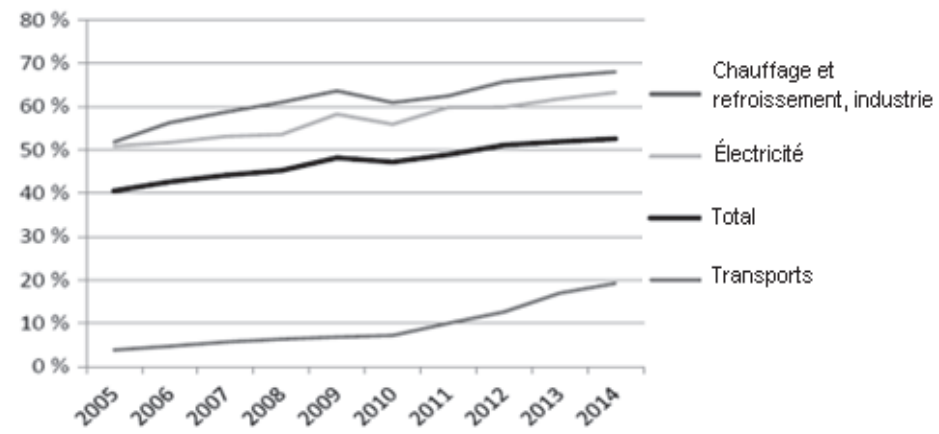

Source : Agence suédoise de l'énergie et Eurostat. 
Le graphique 5 montre la part des énergies renouvelables dans l'approvisionnement énergétique de la Suède. L'objectif de la Suède, adopté par le Parlement suédois dans la loi 2008/2009:162, est d'atteindre 50\% d'énergies renouvelables en 2020. C'est un peu plus que l'objectif fixé par l'UE pour le pays, qui est de $49 \%$. Le graphique montre que le taux de $50 \%$ a été atteint dès 2012 . Aucun nouvel objectif n'a été fixé.

\section{Graphique 6 : Production nette d'électricité (1971-2015, en TWh)}

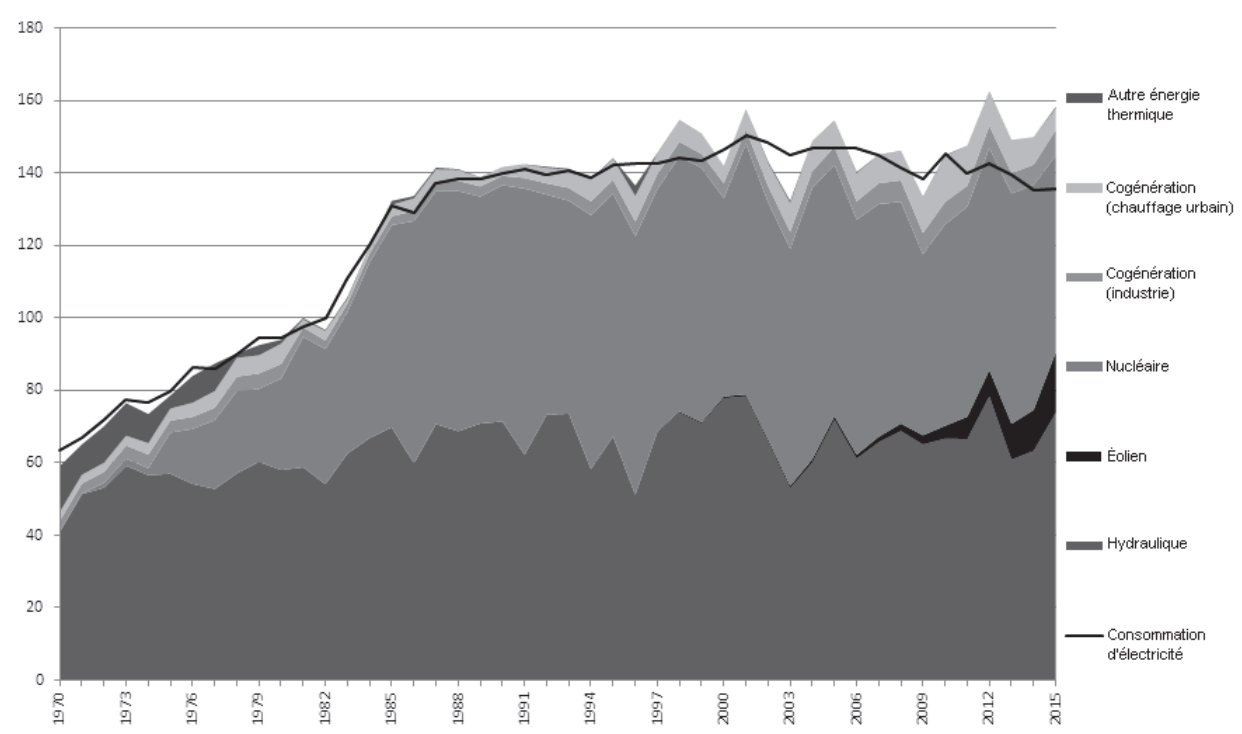

Source : Agence suédoise de l'énergie, Energy in Sweden 2016.

Le graphique 6 montre la production d'électricité. Celle-ci a augmenté au début des années 1980, principalement pour le chauffage électrique, avant de se stabiliser avec un léger déclin ces 15 dernières années. Ces dernières années, les exportations d'électricité ont atteint $20 \mathrm{TWh}$ par an.

L'énergie hydroélectrique et le nucléaire ont assuré la plus grande part de la production, avec une légère croissance de l'hydroélectricité. L'énergie nucléaire a culminé à la fin des années 1980 et décline désormais, au fur et à mesure que les réacteurs vieillissent et que certaines petites centrales sont fermées faute de rentabilité.

La production combinée de chaleur et d'électricité (cogénération) pour le chauffage urbain et l'industrie connaît une croissance lente. L'éolien a commencé à progresser dans la dernière décennie, et représente désormais environ $10 \%$ de la production totale d'électricité.

Afin d'augmenter les investissements dans la production d'électricité renouvelable, un système de certificats «électricité verte » a été mis en place au début des années 2000. Les producteurs d'électricité renouvelable reçoivent 
les certificats verts pour chaque MWh fourni, et les distributeurs sont obligés de les acheter sur un marché. Ce système s'étend aussi à la Norvège depuis 2012. Les capacités de production installées peuvent fournir quelque $20 \mathrm{TWh} / \mathrm{an}$. La plupart des investissements ont été réalisés dans l'éolien et la cogénération biomasse, avec quelques centrales hydroélectriques de petite puissance (cf. graphique 7).

Graphique 7 : Production d'électricité par source d'énergie dans le système de certificats d'électricité (2003-2014, en TWh)

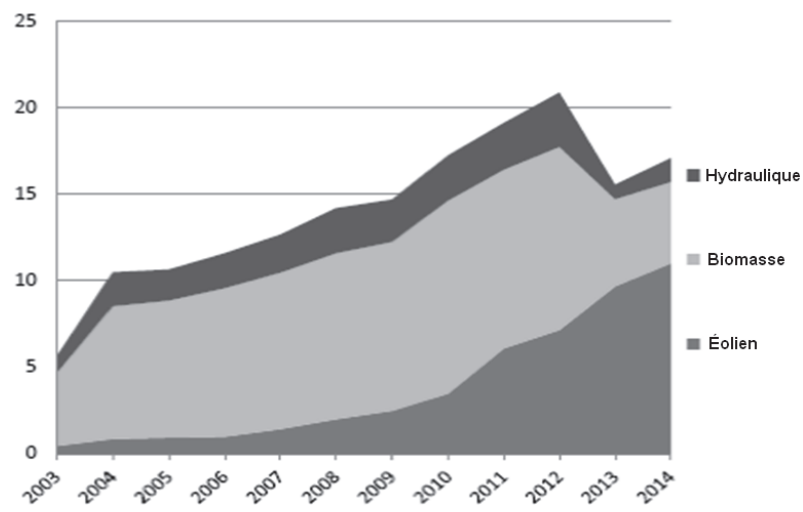

Source : Agence suédoise de l'énergie, Energy in Sweden 2016.

Graphique 8 : Sources d'énergie utilisées dans la production de chauffage urbain (1970-2014, en TWh)

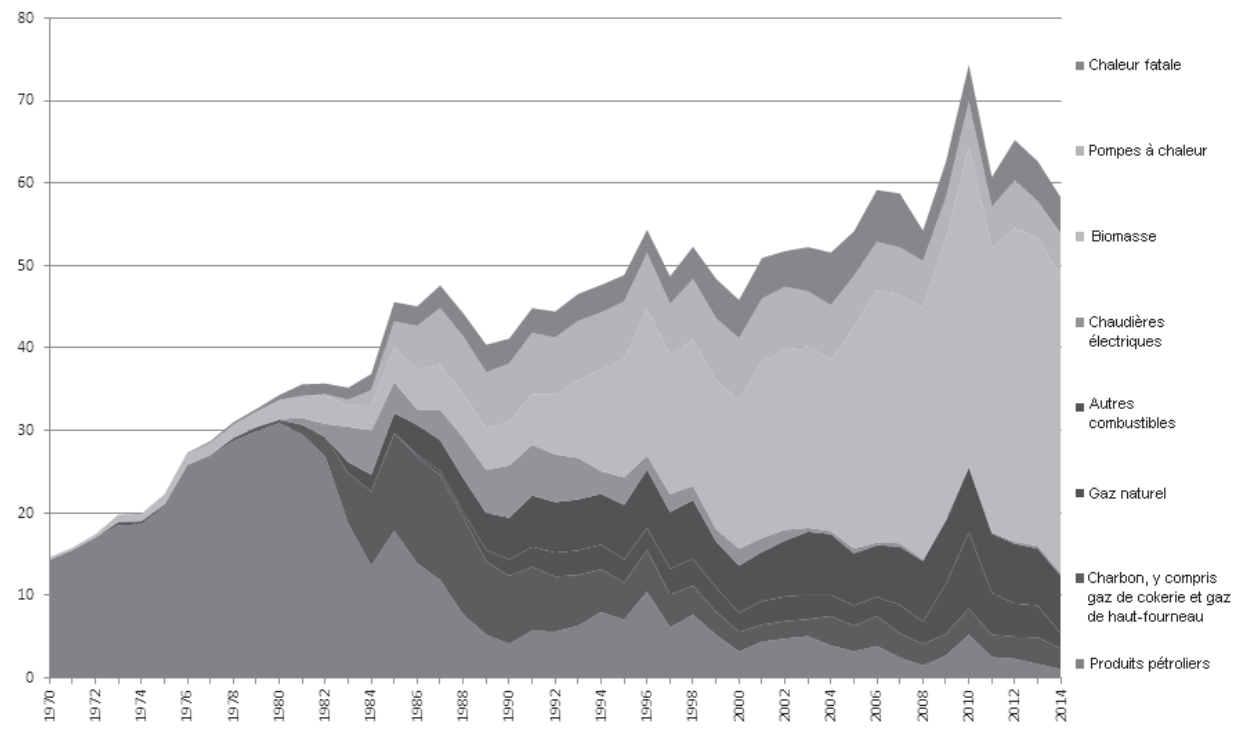

Source : Agence suédoise de l'énergie, Energy in Sweden 2016. 
L'utilisation d'énergie pour le chauffage urbain est présentée dans le graphique 8. Une partie de cette énergie provient de centrales à cogénération. Le chauffage urbain dessert la plupart des logements collectifs et des bâtiments publics, notamment les écoles, les hôpitaux ou les bureaux. L'énergie primaire utilisée dans les centrales à cogénération était principalement le pétrole dans les années 1980, puis on s'est tourné vers le charbon et le gaz naturel, avant de donner à partir des années 1990 une place croissante à la biomasse et à la chaleur fatale. La hausse du prix du pétrole en 1973 et en 1980 est en partie à l'origine de l'usage croissant du charbon et du gaz naturel, tandis que c'est la taxe carbone, introduite en 1991, qui a en partie motivé la transition vers la biomasse. Les combustibles fossiles encore utilisés entrent dans le cadre du SEQE.

\section{Graphique 9 : Échanges nets d'électricité de la Suède (1987-2015, en TWh)}

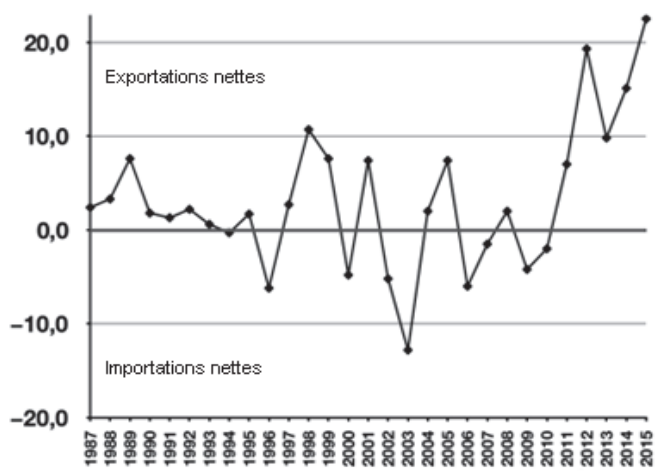

Source : Svensk Energi («Énergie suédoise », organisme de branche).

La Suède dispose de bonnes interconnexions électriques avec les pays nordiques, et les importations/exportations d'électricité ont fluctué au fil des ans, notamment en fonction des variations de disponibilité de l'hydroélectricité. Le déclin progressif de la demande en électricité ces 10 dernières années en Suède et l'expansion de l'éolien et de la cogénération biomasse ont permis aux exportations suédoises d'atteindre environ $20 \mathrm{TWh} / \mathrm{an}$ (cf. graphique 9).

\section{Le secteur des transports}

Les émissions de gaz à effet de serre en Suède proviennent à quelque $33 \%$ du transport routier.

Le projet de loi 2008/2009 sur l'énergie et le climat dispose que la priorité à long terme de la Suède devrait être d'avoir une flotte de véhicules indépendante des combustibles fossiles d'ici 2030. On pourrait voir cet objectif comme une étape vers la vision pour 2050 citée plus haut.

En 2012, une commission a été chargée de proposer des mesures afin de concrétiser cette priorité. La commission a soumis son rapport au gouvernement en décembre 2013, sous la référence SOU 2013:84. 


\section{Graphique 10 : Consommation d'énergie finale} dans le secteur des transports intérieurs (1970-2014, en TWh)

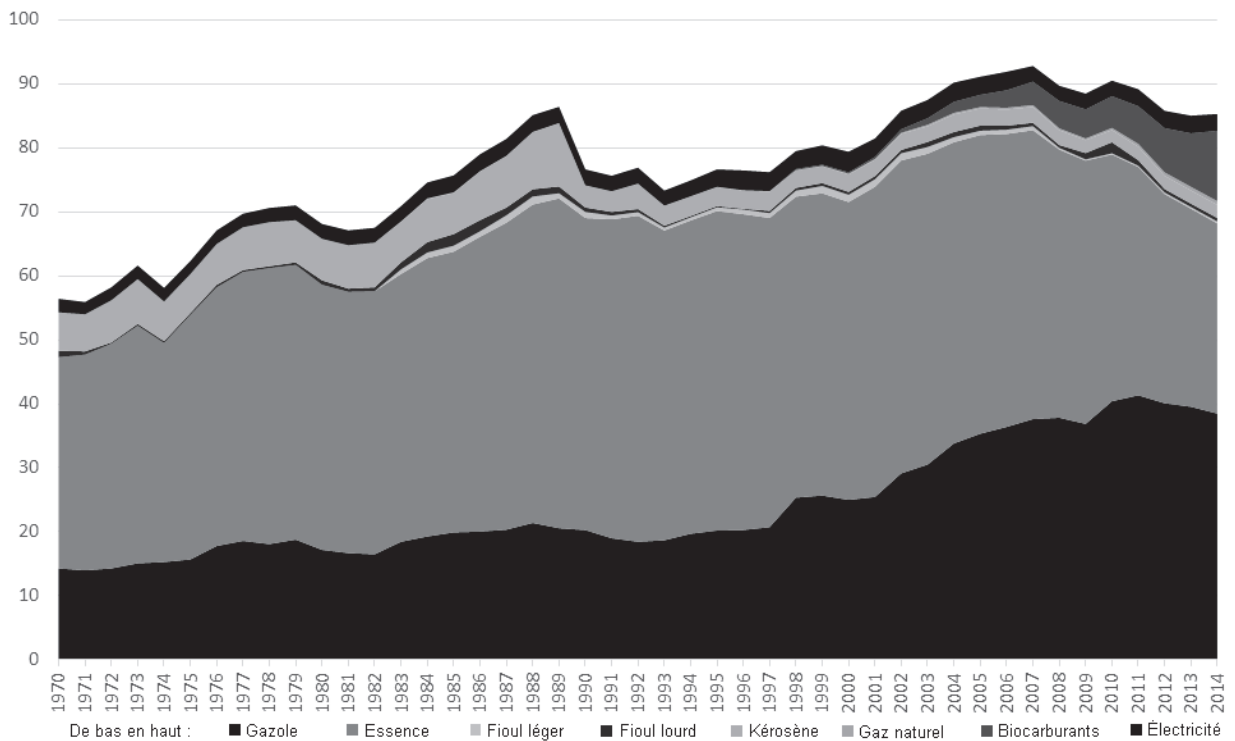

Source : Agence suédoise de l'énergie, Energy in Sweden 2016.

Le graphique 10 montre la consommation d'énergie finale dans le secteur des transports intérieurs. L'usage du gazole a augmenté depuis le début des années 2000, au détriment d'une partie de l'essence. L'électricité concerne le transport ferroviaire.

\section{Graphique 11 : Part des biocarburants dans le secteur des transports (intérieurs)} par rapport au total des carburants dans le secteur du transport routier (2007-2015, en \%)

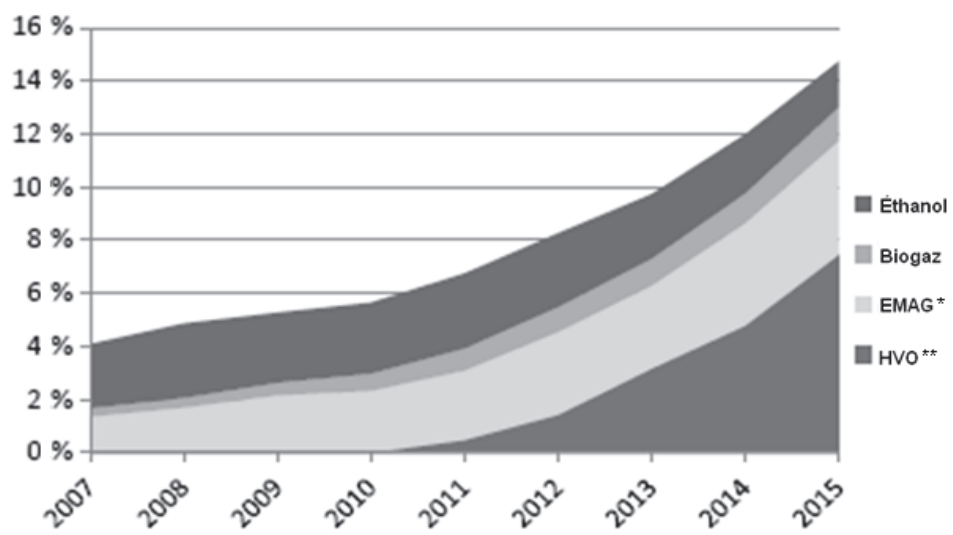

Sources : Agence suédoise de l'énergie et Office suédois de la statistique (SCB). * EMAG : esters méthyliques d'acides gras ; ** HVO : huiles végétales hydrotraitées. 
Les biocarburants ont atteint $14 \%$ du total des carburants dans le secteur du transport routier (cf. graphique 11). Cependant, en utilisant la méthode de calcul introduite dans la directive européenne 2009/28/CE, le pourcentage des énergies renouvelables atteint $24 \%$, ce qui est largement au-dessus des objectifs de l'UE pour la région (cf. graphique 12).

Graphique 12 : Part des biocarburants dans le secteur des transports, calculée selon la méthode indiquée dans la directive européenne 2009/28/CE (en \%)

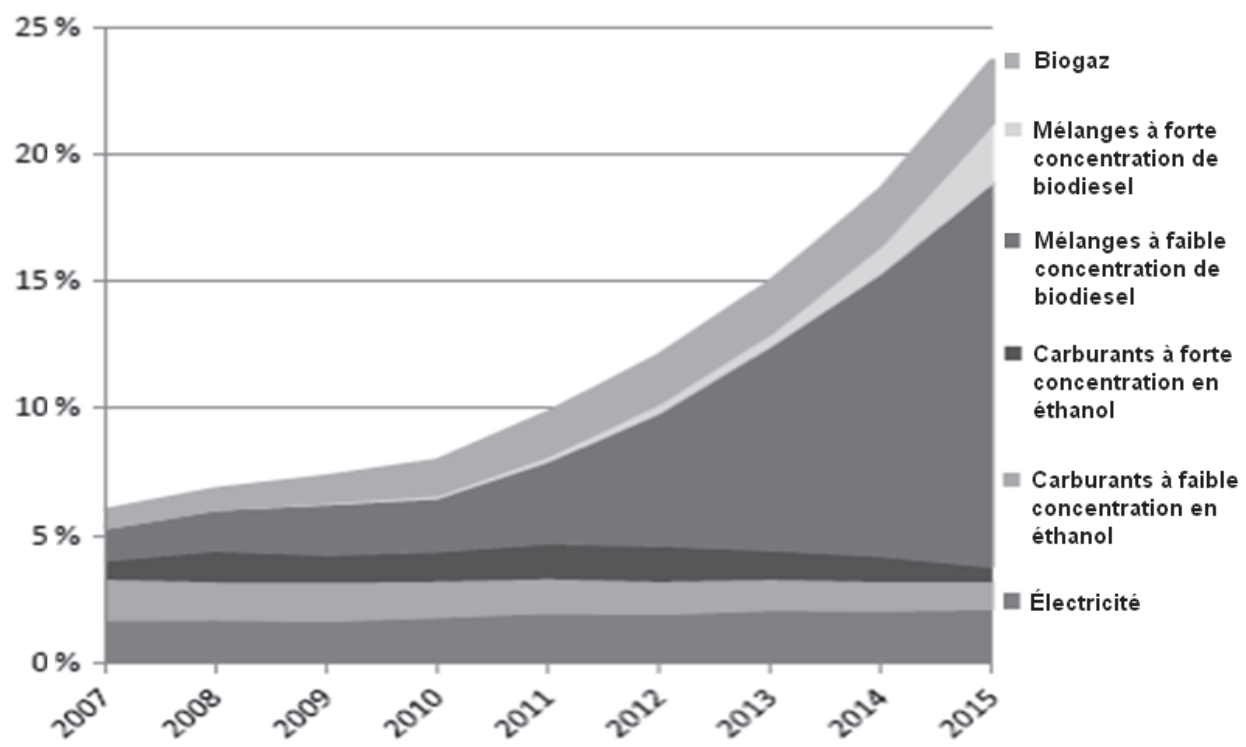

Source : Agence suédoise de l'énergie et Office suédois de la statistique (SCB).

Pour réduire la part des combustibles fossiles dans les transports et atteindre l'objectif présenté dans la loi, la commission a envisagé cinq secteurs dans lesquels on pourrait agir, afin d'étudier à quel point diverses mesures pourraient contribuer à libérer le transport routier des combustibles fossiles :

- la poursuite de la transition sociale vers une réduction des transports avec une augmentation de l'efficacité ;

- les investissements dans les infrastructures et le changement de mode de transport ;

- une meilleure efficacité énergétique des véhicules ;

- un transport routier électrique ;

- les biocarburants.

Le résultat est présenté dans le graphique 13. De presque 80 TWh consommés en 2010, les autorités ont estimé que la demande allait passer à 90 TWh en 2030, puis juste au-dessus de 100 TWh en 2050. Sur la base de ces niveaux, la réduction des combustibles fossiles pourrait être influencée par les cinq mesures 
citées plus haut. Le cas 2030A représente une application intégrale des mesures identifiées, tandis que le cas 2030B illustre une introduction et une pénétration un peu plus lentes de ces mesures. Les deux cas pour 2050 sont générés de la même manière.

Pour 2030, la commission n'a pas trouvé de moyen d'éliminer totalement les combustibles fossiles, ce qui n'est pas surprenant étant donné le nombre de véhicules en circulation, avec une durée de vie moyenne de 17 ans (la mise au rebut précoce des véhicules n'a pas été envisagée en raison du coût que cela implique). Il faut également du temps pour augmenter l'approvisionnement en biocarburants et mettre en circulation des véhicules électriques à grande échelle. Cependant, d'ici 2050 il serait tout à fait possible d'éliminer les combustibles fossiles, et même d'exporter des quantités notables de biocarburants, principalement produits à partir de déchets et de sous-produits de l'industrie forestière.

\section{Graphique 13 : Consommation des combustibles fossiles dans le transport routier, avec et sans les mesures}

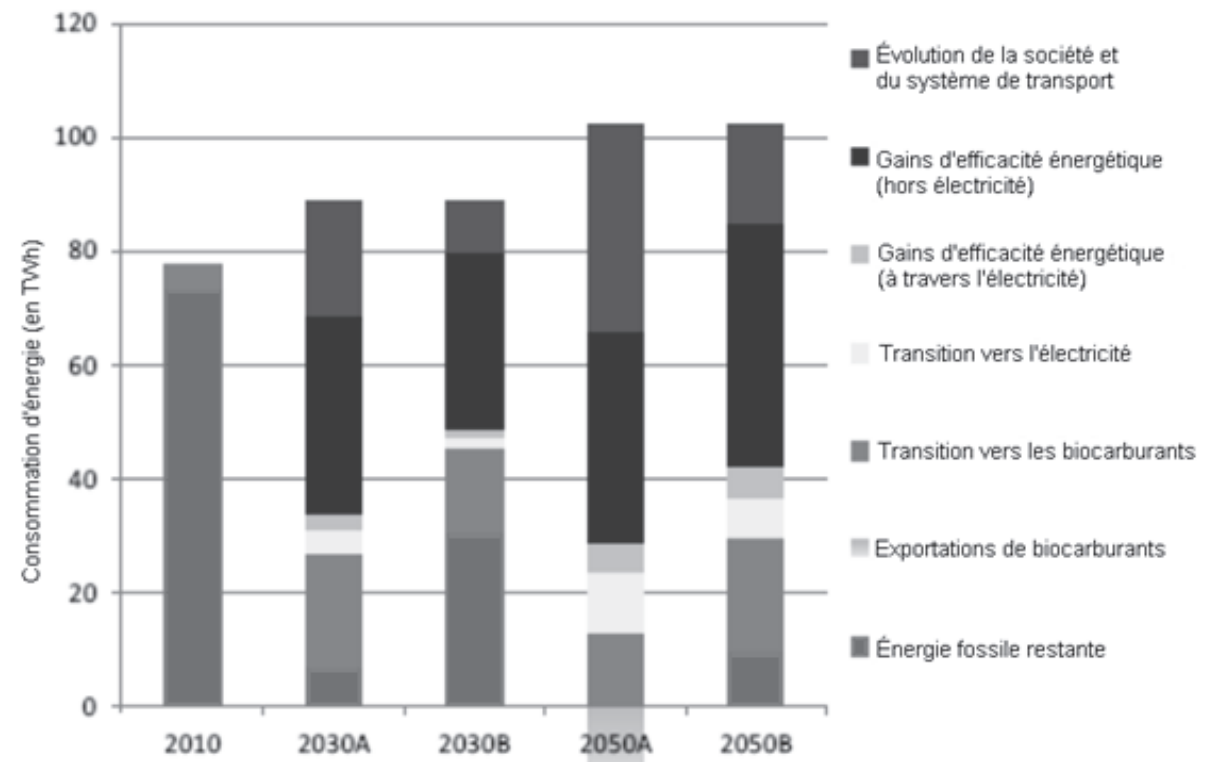

Source : SOU 2013:84.

Les principales mesures consistent en une utilisation plus efficace de l'énergie, en particulier en passant à la motorisation électrique. Sachant que le système électrique de la Suède est pratiquement exempt de combustibles fossiles, cela représente une amélioration de l'efficacité au niveau du système, et donc une réduction significative des gaz à effet de serre ainsi que des autres polluants atmosphériques. 
L'introduction des véhicules électriques pourrait être assez rapide (cf. graphique 14).

\section{Graphique 14 : Moyens de transport par mode de propulsion}

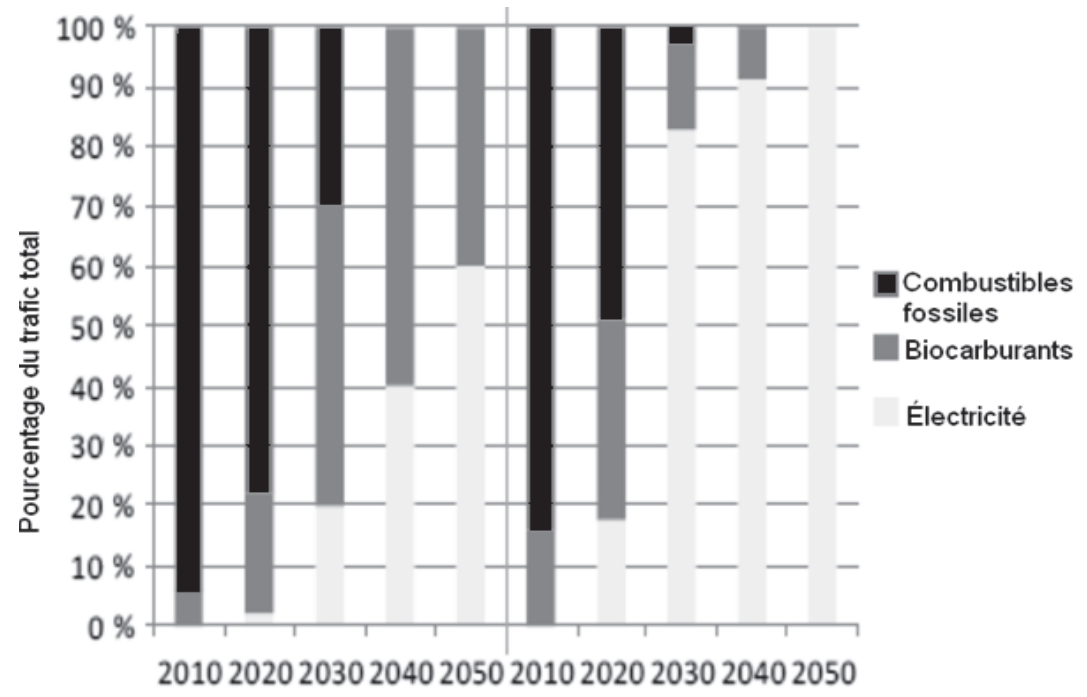

Voitures particulières

Bus urbains/camions de livraison

Source : SOU 2013:84.

La commission a conclu que la vision du gouvernement pour 2030 et 2050 pourrait se réaliser. En outre, lorsqu'on prend du recul pour avoir une vue d'ensemble de la situation, des éléments supplémentaires deviennent visibles. La réalisation de transports routiers sans combustibles fossiles aurait d'autres avantages. Par rapport aux propositions de la commission, ces bénéfices multiples incluent :

- un approvisionnement énergétique plus sûr ;

- de meilleures opportunités d'emploi ;

- une meilleure accessibilité dans les villes ;

- une réduction des émissions de polluants atmosphériques et du bruit ;

- une augmentation de l'activité physique et une amélioration de la santé ;

- une base de transports publics accrue ;

- une réduction des besoins fonciers pour le transport ;

- des progrès en termes d'intégration et d'équité sociales ;

- une meilleure sécurité routière ;

- une réduction des coûts au fil du temps.

Chacun de ces avantages a une valeur importante pour la société, qui n'est pas visible sur le marché. Ensemble, tous ces avantages justifient encore plus la mise en œuvre d'un avenir des transports routiers sans combustibles fossiles. 


\section{Du partage de l'effort au partage des bénéfices?}

Pour atteindre l'objectif de l'accord de Paris et maintenir le réchauffement mondial à moins de $2{ }^{\circ} \mathrm{C}$ au-dessus du niveau préindustriel, il faut procéder à une restructuration immédiate en profondeur des systèmes énergétiques, en abandonnant les combustibles fossiles, afin d'amener les émissions mondiales de gaz à effet de serre à un niveau proche de zéro d'ici 2050. Pour maintenir le réchauffement mondial aussi près que possible de $1,5^{\circ} \mathrm{C}$, il faudrait réduire les émissions encore plus rapidement. Le fait que le monde utilise actuellement presque $80 \%$ de son énergie primaire sous forme de combustibles fossiles illustre bien l'ampleur du défi. L'abandon des combustibles fossiles est souvent présenté comme une évolution extrêmement coûteuse. Lors des négociations sur la lutte contre le réchauffement climatique, la notion de "partage de l'effort» repose sur cette notion de coût élevé associé à l'atténuation du réchauffement climatique. Heureusement, le coût des énergies renouvelables diminue rapidement, et dans de nombreuses situations, leur coût conventionnel est déjà inférieur à celui des alternatives conventionnelles (combustibles fossiles).

Cependant, l'introduction de la notion de «bénéfices multiples » offre une approche qui permet d'éviter les connotations négatives liées à la notion de partage de l'effort. Les bénéfices multiples ont été au cœur de l'évaluation énergétique mondiale (Global Energy Assessment, GEA) ${ }^{4}$. L'analyse GEA a montré que tous les grands défis du développement durable liés aux problèmes énergétiques (que ce soit la croissance économique, l'élimination de la pauvreté, la sécurité de l'emploi, le réchauffement climatique, la pollution atmosphérique, l'environnement local, et bien d'autres) pourraient être relevés simultanément en s'attachant d'abord à utiliser l'énergie plus efficacement, en particulier au niveau de la consommation finale, mais aussi à utiliser davantage de sources d'énergie renouvelable.

Cette double approche apporterait bien d'autres avantages à toutes les sociétés, notamment plus d'emplois au niveau local, un soutien de la croissance, une réduction du nombre de personnes vivant dans la pauvreté, un air plus pur à l'intérieur et à l'extérieur - donc moins de nuisances pour la santé -, un approvisionnement énergétique plus sûr, une réduction des risques d'accidents graves et de leur ampleur, une réduction de la dégradation de l'environnement local, une réduction des risques climatiques, etc. Tous ces avantages ne sont pas pris en compte dans les évaluations traditionnelles des coûts de marché.

Cette approche suggère qu'il y a un grand intérêt à changer de point de vue, pour se détourner d'un grand nombre de défis qui semblent peu liés entre eux et se concentrer sur des champs d'action - l'énergie en est un, peut-être le plus important.

L'approche des bénéfices multiples permet de déplacer la discussion, des avantages mondiaux à long terme de la lutte contre le réchauffement climatique

\footnotetext{
${ }^{4}$ Thomas B. Johansson et al. (dir.), Global Energy Assessment, Cambridge University Press, Cambridge, 2012.
} 
vers les avantages locaux à court terme (emploi, santé, éducation...). Puisque les bénéfices multiples sont associés à des valeurs positives, le plus souvent locales, la discussion pourrait passer du partage de l'effort au partage des bénéfices !

On se concentrerait ainsi sur les investissements dans l'efficacité énergétique et les énergies renouvelables, et non sur la limitation du réchauffement climatique. Cette limitation ne serait qu'un des bénéfices obtenus parmi d'autres. Lors des négociations sur le climat, on a employé le terme de cobénéfices, pour indiquer l'existence d'autres avantages que la limitation du réchauffement climatique. Cette manière de présenter de tels bénéfices reste focalisée sur la limitation du réchauffement, en négligeant le fait que les principaux moteurs de la transition vers des investissements dans l'efficacité énergétique et les énergies renouvelables sont différents, par exemple la sécurité ou la qualité de l'air.

Par conséquent, on peut considérer qu'en concentrant les investissements sur l'efficacité énergétique et les énergies renouvelables, on investit dans les instruments du développement durable.

Leur mise en œuvre bénéficierait de la construction de partenariats entre les différents groupes qui profitent des investissements dans l'efficacité énergétique/les énergies renouvelables.

Un pas dans cette direction, pour considérer l'énergie comme un champ d'action, a été franchi dans le cadre des objectifs de développement durable (ODD) adoptés par l'Assemblée générale des Nations unies en septembre 2015. L'objectif 7 est ambitieux, puisqu'il prévoit notamment de garantir un accès universel à des services énergétiques fiables, durables et modernes, à un coût abordable, d'accroître nettement la part des énergies renouvelables dans le bouquet énergétique mondial, de multiplier par deux le taux mondial d'amélioration de l'efficacité énergétique, de renforcer la coopération internationale en vue de faciliter l'accès à la recherche et aux technologies relatives à l'énergie propre en incluant les énergies renouvelables, l'efficacité énergétique et les nouvelles technologies relatives aux combustibles fossiles propres - et de promouvoir l'investissement dans l'infrastructure énergétique et les technologies relatives à l'énergie propre.

CES DERNIÈRES DÉCENNIES, LA SUÈDE a connu la croissance économique et réduit ses émissions de gaz à effet de serre. Cela montre qu'il est possible d'apporter des changements notables. Néanmoins, c'est un processus qui prend du temps : il faut penser en décennies. Il reste toutefois d'importants défis à relever pour le pays. En même temps, les perspectives sont considérables, surtout si l'on regarde au-delà de l'économie conventionnelle. Pour concrétiser ces opportunités, il faut effectivement élargir le point de vue. Les gouvernements doivent agir au-delà de la situation actuelle. 


\section{Les choix énergétiques d'autres pays européens : la Pologne ${ }^{1}$}

\section{Marek WASIŃSKI}

Les choix d'un gouvernement en matière de politique énergétique sont étroitement liés au parcours historique du pays, car le secteur énergétique ne peut pas s'organiser au jour le jour : il nécessite des investissements à long terme et a un fort impact sur l'emploi. C'est précisément le cas de la Pologne, où les décisions prises par le gouvernement sous contrôle communiste, qui a poussé au développement de l'industrie lourde et des centrales à charbon, pèsent toujours sur la réflexion actuelle en matière de politique énergétique. La transformation d'une économie homogène basée sur le charbon sera inévitablement associée à un coût social et à d'importants investissements. D'un autre côté, l'absence de cette transformation signifierait que ce coût deviendrait encore plus élevé à long terme. Nous présentons dans cet article les possibilités existantes pour les futurs choix énergétiques de la Pologne.

\section{Sécurité énergétique}

À partir des discussions des décideurs polonais sur l'énergie, on peut identifier les principaux facteurs qui les motivent : l'indépendance énergétique (problème de l'importation des ressources et de la technologie), un approvisionnement stable et économique en énergie, la réduction des prix pour les particuliers, la limitation des impacts négatifs de la transformation sur les emplois, et l'adaptation aux règles climatiques européennes. D'une manière légèrement différente, ces principes ont été définis dans le Plan de développement responsable, document stratégique du ministère polonais du Développement économique. Les objectifs fixés pour les 10 ans à venir sont les suivants : la stabilité de l'approvisionnement énergétique, la baisse de la consommation d'énergie primaire, l'augmentation graduelle et rentable de la part des sources d'énergie renouvelable (SER) dans le mix énergétique, et la limitation de l'augmentation du prix de l'énergie pour les ménages. La condition sine qua non de la compétitivité, pour l'économie polonaise, est son objectif de «moderniser le secteur énergétique et de diversifier les sources d'énergie ${ }^{2} »$.

Apparemment, tous ces principes peuvent se résumer en un seul : la sécurité énergétique. Dans sa définition par l'Agence internationale de l'énergie (AIE), cette sécurité inclut la stabilité de l'approvisionnement et du coût de l'énergie pour les consommateurs ${ }^{3}$. Cependant, la sécurité est une notion plus large, car

\footnotetext{
${ }^{1}$ Article rédigé en janvier 2017, NdE.

${ }^{2}$ Ministère du Développement économique de la République de Pologne, Strategia na rzecz Odpowiedzialnego Rozwoju, Varsovie, 29 juillet 2016.

3 «L'AIE définit la sécurité énergétique comme la disponibilité ininterrompue de sources d'énergie à un prix abordable. La sécurité énergétique a différents aspects : la sécurité énergétique à long terme est principale-
} 
elle inclut également les défis climatiques ainsi que les risques sanitaires, comme dans le cas des catastrophes de Fukushima ou Tchernobyl. Les scientifiques polonais, dans ce domaine, soulignent également d'autres aspects de la sécurité énergétique, liés à l'autonomie économique et à la minimisation des éventuelles pressions politiques exercées par les entités de vente et de transit (sociétés privées ou autres pays) ${ }^{4}$. Avec une définition aussi large, les discussions et les choix énergétiques de la Pologne sont principalement motivés par le souci de sa sécurité énergétique.

\section{Indépendance énergétique}

L'indépendance dans le domaine de l'énergie peut être considérée de plusieurs points de vue. Premièrement, c'est le ratio entre importations et sources intérieures d'énergie. Deuxièmement, c'est la diversification des fournisseurs. Récemment, la Pologne dépendait essentiellement d'un seul pays fournisseur pour ses importations de gaz et de pétrole : la Russie. En 2014, elle a importé $98 \%$ de son pétrole et $66 \%$ de son gaz ${ }^{5}$. Cependant, grâce aux efforts des décideurs, cette dépendance a pu être réduite. Le terminal gazier de Świnoujście, ouvert en 2016, a la capacité de fournir à la Pologne un tiers de sa consommation de gaz, et il existe des possibilités d'extension future ${ }^{6}$. Cette année également a eu lieu le premier achat de pétrole à l'Arabie saoudite. Après les actions russes sur le marché du gaz en 2012, puis l'agression de la Russie avec l'occupation de la Crimée et de l'est de l'Ukraine, la diversification des fournisseurs est devenue une priorité pour les gouvernements polonais. Ce n'était d'ailleurs pas le cas que pour la Pologne, mais pour le marché européen dans son ensemble.

Troisièmement, l'indépendance énergétique concerne non seulement les importations, mais aussi la diversité des ressources énergétiques. Dans ce domaine, on peut observer une forte dépendance au charbon. En 2014, le lignite et la houille représentaient plus de $50 \%$ de la consommation d'énergie primaire

ment une question d'investissements effectués en temps et en heure pour fournir de l'énergie en phase avec le développement économique et les besoins environnementaux. Par ailleurs, la sécurité énergétique à court terme se préoccupe de la capacité du système énergétique à réagir rapidement à des changements soudains dans l'équilibre entre l'offre et la demande » (AIE, http://www.iea.org/topics/energysecurity/, consulté le 30/05/2017).

${ }^{4}$ Maciej Janowski, Marcin Jastrzębski, Łukasz Nowakowski, Igor Protasowicki, Polityka bezpieczeństwa energetycznego państw Europy Środkowo-Wschodniej. Rola i znaczenie Grupy Wyszehradzkiej, Towarzystwo Naukowe Powszechne, Varsovie, 2016, p. 17-48; Zdzisław Nowakowski, Polityka bezpieczeństwa energetycznego Polski. Wptyw europeizacji i globalizacji, Towarzystwo Naukowe Powszechne, Varsovie, 2016, p. 7-13, $45-65$.

${ }^{5}$ Office central de la statistique (GUS).

6 Michał Duszczyk, «Polska znacznie zwiększa import ropy naftowej spoza Rosji », Rzeczpospolita, 5 septembre 2016, p. [n.c.] ; Sylwia Zadrozna, « Terminal LNG w Świnoujściu: dziś pierwsza dostawa » [en ligne], PolskieRadio, 17 juin 2016, p. [n.c.]. Disponible sur: http://www.polskieradio.pl/42/273/Artykul/ 1632761,Terminal-LNG-w-Swinoujsciu-dzis-pierwsza-dostawa [consulté le 30/05/2017]. 
en Pologne, tandis que le pétrole comptait pour $25 \%$, le gaz pour $13 \%$ et les énergies renouvelables pour un peu plus de $11 \%{ }^{7}$.

\section{Graphique 1 : Consommation d'énergie primaire en 2013 (en \%)}

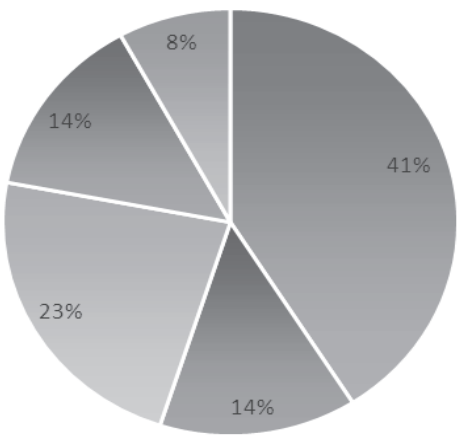

Source : Office général de la statistique de Pologne.

La dépendance de l'économie polonaise envers le charbon est encore plus forte quand on étudie le mix énergétique de la production d'électricité. Plus de $50 \%$ de l'électricité était produite à partir de centrales alimentées à la houille, $35 \%$ au lignite, plus de $12 \%$ provenait de SER et $2 \%$ du gaz ${ }^{8}$.

\section{Graphique 2 : Production d'électricité en 2014 (en \%)}

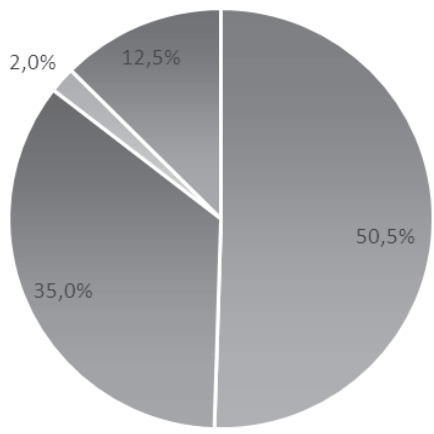

Source : Office général de la statistique de Pologne.

\footnotetext{
${ }^{7}$ Jacek Kamiński, Przemysław Kaszyński, Marcin Malec, Adam Szurlej, « Analiza zmian zużycia energii pierwotnej w Polsce w kontekście liberalizacji rynków paliw i energii », Polityka Energetyczna - Energy Policy Journal, $\mathrm{n}^{\circ}$ 3, 2015, p. 25-36.

${ }^{8}$ Office central de la statistique (GUS).
} 
Bien qu'on extraie du charbon en Pologne et que les réserves soient estimées à au moins 57 ans $^{9}$, l'instabilité du marché ainsi que la hausse des coûts d'extraction et des émissions imposent à long terme de diversifier les sources pour assurer un approvisionnement stable et économique.

\section{Nécessité de transformation}

Le marché polonais de l'électricité est confronté au défi de la mise hors service des vieilles centrales, soit $21 \mathrm{GW}$ dans les 15 prochaines années ${ }^{10}$. Presque $60 \%$ de toutes les turbines électriques ont plus de 30 ans, auxquelles s'ajoutent $16 \%$ qui ont plus de 20 ans. Des investissements sont en cours pour la modernisation et la construction de nouvelles tranches dans les centrales à charbon d'Opole, Kozienice et Jaworzno, mais il en faudra plus pour combler les lacunes dans les prochaines années. Les lignes de transport posent un défi similaire. En effet, $80 \%$ des lignes à $220 \mathrm{kV}, 23 \%$ des lignes à $400 \mathrm{kV}$ et $38 \%$ des transformateurs ont plus de 30 ans. À cause de l'âge avancé de ces infrastructures, les pertes moyennes en ligne dépassent $8 \%$ en Pologne, alors que la moyenne de l'UE se situe autour de $5 \%{ }^{11}$. Cela nécessite également des investissements de modernisation du système énergétique polonais.

\section{Graphique 3 : Prix de l'électricité pour les particuliers au $2^{\mathrm{e}}$ semestre (S2) de l'année 2015 (en euros/KWh)}

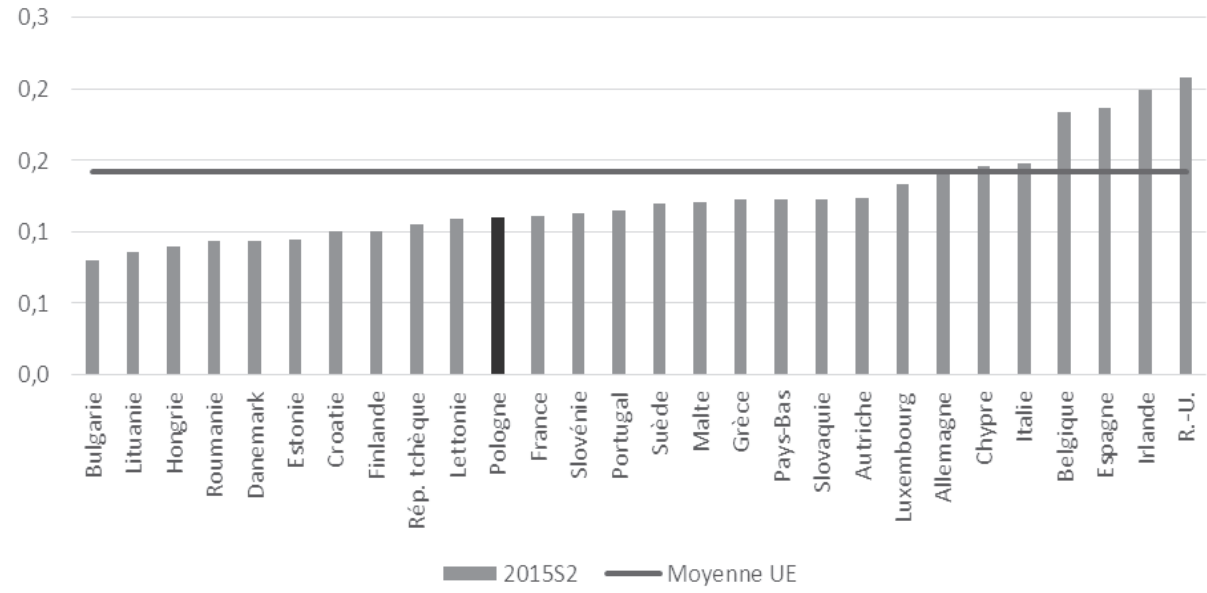

Source : Eurostat.

\footnotetext{
${ }^{9}$ Maciej Janowski, Marcin Jastrzębski, Łukasz Nowakowski, Igor Protasowicki, op. cit., p. 54.

${ }^{10}$ [s.a.], «Forum Energii: Rynek mocy nie pomoże w transformacji energetyki » [en ligne], Biznes Alert, 28 octobre 2016, p. [n.c.]. Disponible sur: http://biznesalert.pl/forum-energii-rynek-mocy-nie-pomoze-wtransformacji-energetyki/ [consulté le 30/05/2017].

${ }^{11}$ Strategia na rzecz Odpowiedzialnego Rozwoju, op. cit.
} 
Par rapport aux autres États membres, les tarifs nominaux de l'électricité en Pologne sont inférieurs à la moyenne européenne pour les consommateurs industriels et privés, et légèrement supérieurs dans le cas du gaz. D'un autre côté, ces prix ajustés au pouvoir d'achat constituent une charge trop lourde. Il existe plusieurs définitions de la précarité énergétique. Si nous utilisons le seuil de $10 \%$ du budget d'un ménage, $44 \%$ des Polonais pourraient faire partie de ce groupe. Si on déplace ce seuil à $13 \%$, un tiers de la population du pays pourrait être considérée en situation de précarité énergétique. Selon un autre modèle, l'indicateur "Low Income High Costs », l'Institut pour le développement durable (Instytut na rzecz Ekorozwoju) est arrivé à la conclusion que $17 \%$ des Polonais étaient confrontés à ce problème. Cela signifie qu'un cinquième de la population polonaise ressent fortement le poids du coût de l'énergie dans son budget ménager ${ }^{12}$.

\section{Graphique 4 : Prix de l'électricité pour les particuliers au 2e semestre (S2) de l'année 2015, standard de pouvoir d'achat (en euros/KWh)}

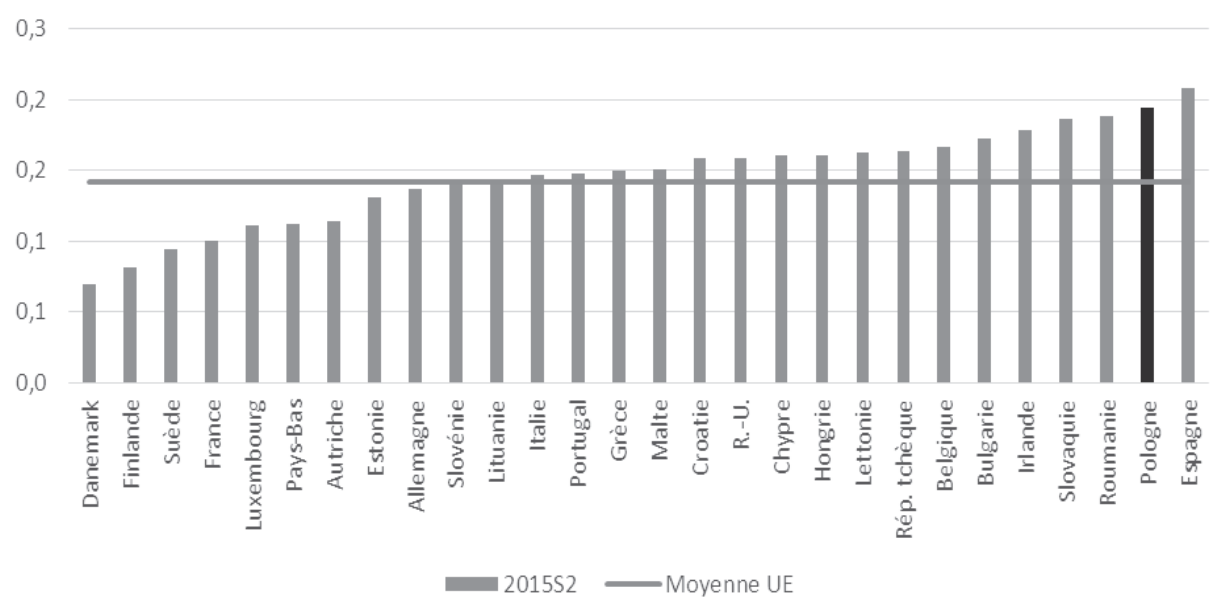

Source : Eurostat.

\section{Politique climatique}

La forte dépendance au charbon mentionnée plus haut constitue un grand défi pour les décideurs polonais en matière de politique climatique également. Les investissements historiques dans ce secteur ont laissé une industrie marquée par d'importantes émissions de carbone et une forte consommation d'énergie. Nominalement, les économies d'Europe centrale et orientale sont plus gourmandes en énergie : par exemple, en 2013 la Pologne a consommé 2,5 fois plus d'éner-

\footnotetext{
${ }^{12}$ Dominik Owczarek, Agata Miazga, Ubóstwo energetyczne w Polsce - definicja i charakterystyka spoteczna grupy, Instytut na rzecz Ekorozwoju, Varsovie, 2015.
} 
gie que l'Allemagne ${ }^{13}$. Cependant, l'intensité énergétique estimée en parité de pouvoir d'achat (PPA) et corrigée des variations climatiques en 2014 a montré que la différence était nettement moins importante, avec seulement $15 \%$ d'énergie consommée en plus par l'économie polonaise que par celle de l'Allemagne ${ }^{14}$. C'est pour cela que l'amélioration de l'efficacité énergétique est l'un des principaux objectifs visés par les actions de la Pologne en matière de politique climatique.

La chute de l'économie socialiste planifiée a été fortement liée à la régression de l'industrie lourde, ce qui a également entraîné une importante réduction des émissions de gaz à effet de serre (GES). En prenant 1988 comme année de base (comme dans le protocole de Kyoto), la Pologne a réduit de $33 \%$ ses émissions de GES en 2014. Cependant, par rapport à 1990 ou 2005, lorsqu'on exclut les efforts de transformation de l'économie polonaise, la réduction se limite à $20 \%$ et $4 \%$ respectivement.

\section{Graphique 5 : Réduction des émissions de GES en 2014 par rapport à 1988 (en \%)}

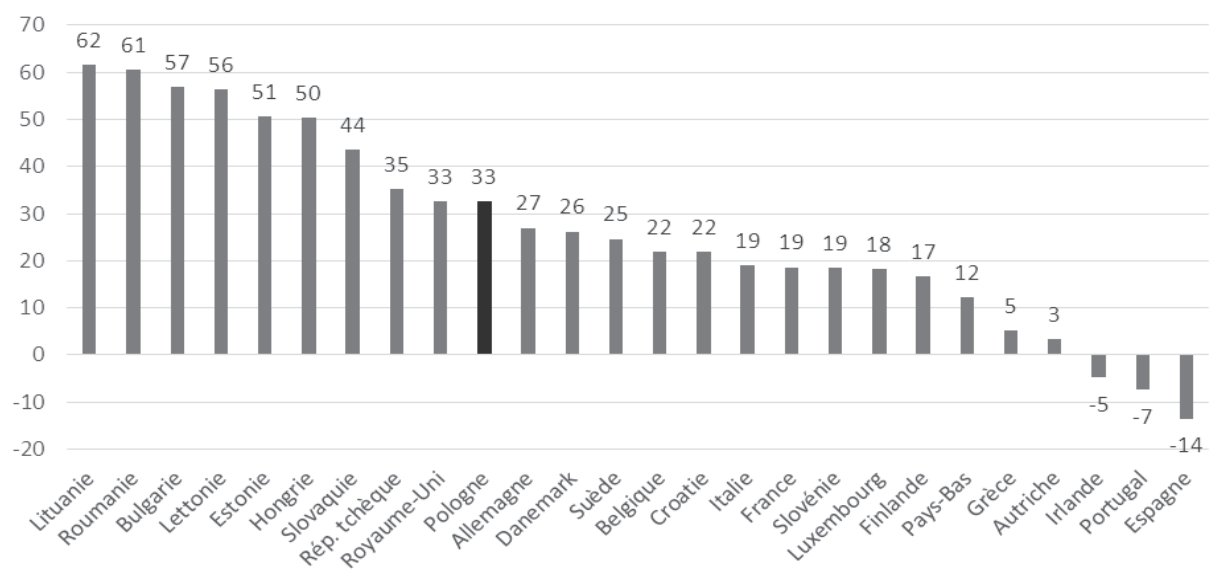

Source : Agence européenne pour l'environnement.

Le gouvernement polonais a signé l'accord de Paris et l'a ratifié le 7 octobre 2016, car il soutient largement le processus international inclusif dans lequel des pays du monde entier contribuent aux efforts de lutte contre le réchauffement de la planète. S'agissant d'une économie basée sur le charbon, l'objectif du gouvernement polonais est d'utiliser tout l'éventail des actions climatiques possibles: pas uniquement les politiques de décarbonation, mais aussi le développement des capacités d'absorption de $\mathrm{CO}_{2}$ dans les puits de carbone naturels comme les forêts.

\footnotetext{
${ }^{13}$ Produit intérieur brut (PIB) à prix constants 2000 (en kg équivalent pétrole/euro), source : Eurostat.

${ }^{14} \mathrm{PIB}$ à prix constants (PPA) 2005, source : Odyssee.
} 


\section{Choix énergétiques pour l'avenir}

L'état du secteur énergétique polonais tel qu'on vient de le décrire avec son besoin de modernisation, la politique climatique européenne et la lutte pour assurer la stabilité de l'approvisionnement énergétique, ainsi que les efforts pour atteindre l'indépendance énergétique montrent clairement que, dans les prochains mois ou les prochaines années, le gouvernement polonais devra faire ses choix politiques afin de mettre en place une stratégie à long terme pour le secteur énergétique. En août 2015, le gouvernement a présenté un document analysant ces possibilités : le plan de politique énergétique polonais PEP2050. Il met en évidence les principaux défis à relever et propose trois scénarios pour le futur mix énergétique de la Pologne. Deux des hypothèses utilisées pour ces scénarios sont extrêmement importantes. La première concerne la demande en énergie. Le plan PEP2050 prédit une croissance régulière jusqu'en 2030, alors que ces dernières années, les prévisions de ce genre se sont toujours avérées trop élevées par rapport à l'augmentation réelle de la demande. Deuxièmement, le mix énergétique prévu pour 2050 sera déjà atteint en 2030, ce qui ne donnerait aucune flexibilité pour les changements technologiques et l'évolution de la demande ${ }^{15}$.

Le plan PEP2050 propose trois scénarios. L'un des principaux facteurs déterminants pour la composition du mix énergétique polonais est la construction de centrales nucléaires. Le programme nucléaire du pays est actuellement suspendu, mais d'après le ministère de l'Énergie, une décision sur la construction du premier réacteur nucléaire pourrait intervenir avant la fin de la législature actuelle $^{16}$. Dans le scénario nucléaire du PEP2050, la consommation d'énergie primaire d'origine nucléaire atteindrait $45 \%$.

\section{Graphique 6 : Consommation d'énergie primaire en 2050, scénario nucléaire (en \%)}
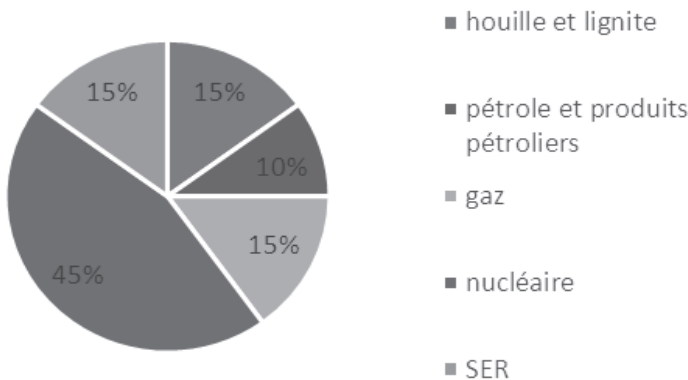

Source : PEP2050.

\footnotetext{
${ }^{15}$ Ministère de l'Économie, Polski Plan Energetyczny 2050, Varsovie, 10 août 2015.

${ }^{16}$ [s.a.], Elektrownia atomowa jednak powstanie. «Decyzja jeszcze w tej kadencji » [en ligne], Forsal.pl, 7 septembre 2016, p. [n.c.]. Disponible sur : http://forsal.pl/artykuly/973810,elektrownia-atomowa-jednakpowstanie-decyzja-jeszcze-w-tej-kadencji.html [consulté le 30/05/2017].
} 
Un scénario plus plausible, en cas de développement d'une centrale nucléaire, serait celui qui était considéré comme le scénario de base en 2015. Il prévoit que l'énergie nucléaire représentera $14 \%$ de la consommation d'énergie primaire en 2050. La houille et le lignite resteront la source d'énergie principale, tandis que le reste de la consommation proviendra de sources diverses.

\section{Graphique 7 : Consommation d'énergie primaire en 2050, scénario de base (en \%)}
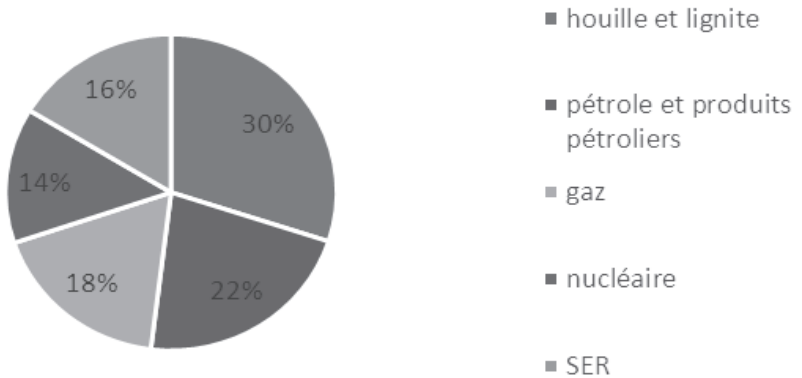

Source : PEP2050.

Le dernier scénario a été élaboré au cas où la décision serait prise de ne construire aucune centrale nucléaire, ce qui nécessiterait une part plus importante d'autres sources d'énergie faiblement émettrices, comme les SER et le gaz. Le gaz offrirait au système la flexibilité nécessaire pendant les périodes de faible production par les SER. Le charbon resterait une source importante d'énergie, au même plan que dans le scénario de base.

\section{Graphique 8 : Consommation d'énergie primaire en 2050, scénario gaz \& SER (en \%)}

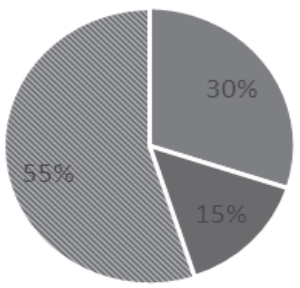

- houille et lignite

- pétrole

gaz \& SER

Source : PEP2050.

En 2016, l'Institut Jagielloński a préparé une autre analyse des choix énergétiques possibles pour le gouvernement polonais ${ }^{17}$. À partir des hypothèses du

\footnotetext{
${ }^{17}$ Wojciech Jakóbik, Bartosz Bieliszczuk, Krystian Kowalewski, Adam Stolarz, Jaka energia będzie napędzać polskq gospodarkę?, Instytut Jagielloński, Varsovie, juin 2016.
} 
plan PEP2050, il a défini trois scénarios. Contrairement au PEP2050, ceux-ci ne montrent que le mix énergétique de la production d'électricité à l'horizon 2030.

Dans le scénario charbon, cette ressource reste la principale source de production d'électricité, en baisse de $10 \%$ par rapport au mix énergétique actuel. La différence provient principalement de la croissance des centrales à gaz, moins de celle des énergies renouvelables. Dans ce scénario, aucune centrale nucléaire ne sera construite.

\section{Graphique 9 : Scénario charbon 2030}

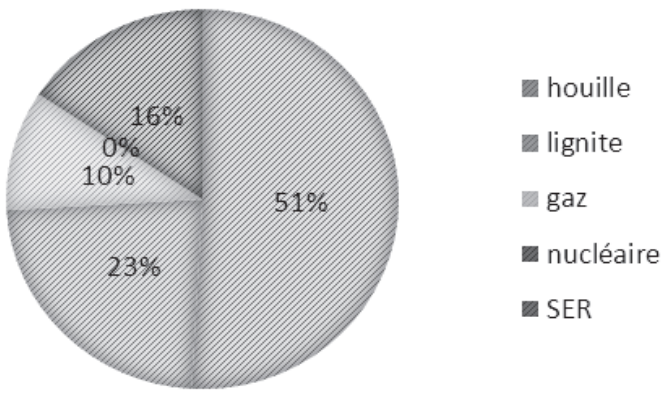

Source : Instytut Jagielloński.

La seule différence entre le scénario charbon et le scénario gaz fournis par l'Institut Jagielloński est la part supérieure du gaz aux dépens de la houille. De cette manière, la Pologne réduirait les émissions en modernisant les centrales à charbon et la technologie du gaz combustible fossile.

\section{Graphique 10 : Scénario gaz 2030}

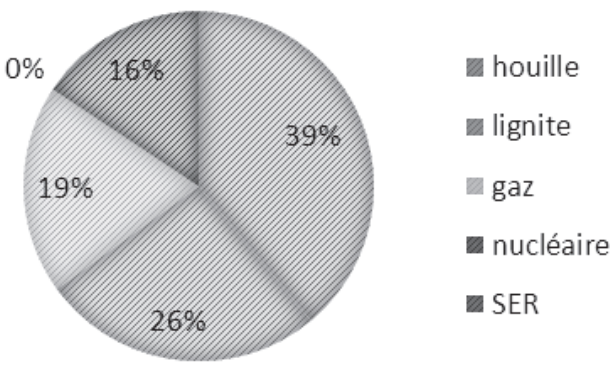

Source : Instytut Jagielloński.

Enfin, le troisième scénario analysé par l'Institut Jagielloński comporte une grande diversité de sources d'électricité, qui permet également une réduction 
accrue des émissions. Au lieu des $16 \%$ prévus, les SER compteraient pour $31 \%$ de la production en 2030 , et les centrales à gaz produiraient $11 \%$ de l'électricité. Une centrale nucléaire se substituerait à des centrales à charbon, pour atteindre $12 \%$ du mix énergétique. La houille et le lignite resteraient la principale source d'électricité, mais tomberaient sous la barre des $50 \%$.

\section{Graphique 11 : Scénario basses émissions 2030}

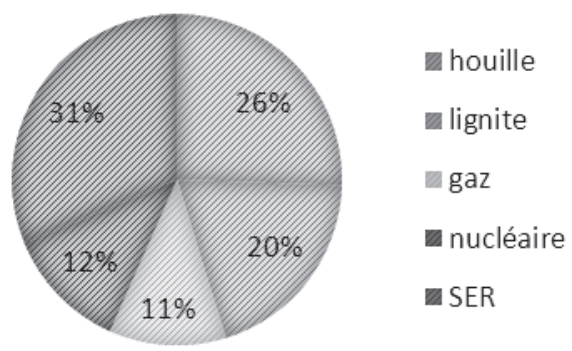

Source : Instytut Jagielloński.

QUELS QUE SOIENT LES CHOIX DES GOUVERNEMENTS POLONAIS dans les années à venir, un facteur déterminant pour la transformation du système énergétique de la Pologne sera l'amélioration de l'efficacité énergétique et la réalisation d'économies d'énergie, donc une transformation des habitudes sociales. Comme on l'a vu dans les scénarios ci-dessus, le charbon restera l'une des principales sources d'énergie du pays, mais sa part dans la production diminuera. C'est pourquoi la modernisation des centrales électriques pour les rendre plus efficaces et le développement de nouvelles technologies moins émettrices seront très importants. Le programme polonais d'e-mobilité va dans la même direction, avec l'objectif de stimuler la croissance économique ${ }^{18}$. Il inclut la production de voitures électriques et le développement d'un réseau électrique suffisant et de centrales électriques ${ }^{19}$. Ce sont des orientations qui accompagneront inévitablement les nouveaux choix de politique énergétique de la Pologne dans les années à venir.

Traduction de Marie-Céline GEORG

\footnotetext{
${ }^{18}$ Pour plus d'informations sur la manière dont la politique énergétique devrait également refléter la politique industrielle, cf. Leszek Jesień, Michał Kurtyka, New Electricity and New Cars. The Future of the European Energy Doctrine, CeDeWu, Varsovie, 2016, p. 63-70.

${ }^{19}$ Strategia na rzecz Odpowiedzialnego Rozwoju, op. cit.
} 


\section{LES AUTEURS}

\section{Stefan C. AYKUT}

Politiste et sociologue au Laboratoire interdisciplinaire sciences innovations sociétés (LISIS), université Paris-Est Marne-la-Vallée et chercheur associé au Centre Marc Bloch de Berlin (CNRS/Université Humboldt de Berlin)

\section{Alain BELTRAN}

Directeur de recherche au CNRS, Unité mixte de recherches (UMR) Sorbonne-Identités, relations internationales et civilisations de l'Europe (SIRICE), université Paris 1 Panthéon-Sorbonne

\section{Ulrich BENTERBUSCH}

Directeur général de l'Agence allemande de l'énergie (Deutsche Energie-Agentur, dena) (jusqu'en 2015)

Actuellement directeur adjoint de la division Chaleur et efficacité énergétique dans l'industrie et les bâtiments résidentiels, ministère fédéral de l'Économie et de l'Énergie, Berlin

\section{Ian COCHRAN}

Directeur de programme Finance, investissement et climat à I4CE (Institute for Climate Economics), Paris

\section{Michel COLOMBIER}

Co-fondateur et directeur scientifique de l'Institut du développement durable et des relations internationales (Iddri) à Paris et professeur associé à Sciences Po Paris

\section{Michel CRUCIANI}

Chargé de mission au Centre de géopolitique de l'énergie et des matières premières (CGEMP) de l'université Paris-Dauphine et chercheur associé à l'Institut français des relations internationales (Ifri), Paris

\section{Joseph DUTTON}

Chercheur associé au groupe Politique énergétique de l'université d'Exeter, Royaume-Uni

\section{Christian EGENHOFER}

Chercheur, responsable du programme Énergie et climat au Centre d'études politiques européennes (Centre for European Policy Studies, CEPS) à Bruxelles

\section{Manfred FISCHEDICK}

Vice-président de l'Institut sur le climat, l'environnement et l'énergie de Wuppertal (Wuppertal Institut)

\section{Severin FISCHER}

Chercheur au département UE/Europe de la Fondation Science et politique (Stiftung Wissenschaft und Politik, SWP) à Berlin (jusqu'en 2015) Actuellement chercheur au sein de l'équipe Sécurité mondiale du Centre d'études sur la politique de sécurité (Center for Security Studies, CSS) de Zurich 


\section{Berthold GOEKE}

Directeur de la sous-direction Politique de lutte contre le changement climatique, ministère fédéral de l'Environnement, Berlin

\section{Hadrien HAINAUT}

Chef de projet Finance, investissement et climat à I4CE (Institute for Climate Economics), Paris

\section{Ulrike HANSEN}

Chef du bureau Monitoring du marché énergétique, Agence fédérale des réseaux

(Bundesnetzagentur), Bonn

\section{Solène HAZOUARD}

Ingénieur d'études au Centre d'information et de recherche sur l'Allemagne contemporaine (CIRAC), Cergy-Pontoise

\section{Thomas B. JOHANSSON}

Professeur émérite et ancien directeur de l'Institut international d'économie environnementale industrielle (The International Institute for Industrial Environmental Economics, IIIEE) de l'université de Lund, Suède, ancien vice-président de l'Évaluation énergétique mondiale (Global Energy Assessment) de l'Institut international d'analyse des systèmes appliqués (International Institute for Applied Systems Analysis, IIASA) de Laxenbourg en Autriche et ancien membre de la commission Flotte de véhicules indépendante des combustibles fossiles au ministère suédois de l'Énergie à Stockholm

\section{Joachim LANG}

Directeur du bureau E.ON SE à Berlin (jusqu'en 2016)

Actuellement directeur général de la Fédération de l'industrie allemande (Bundesverband der deutschen Industrie, BDI), Bruxelles

\section{René LASSERRE}

Directeur du Centre d'information et de recherche sur l'Allemagne contemporaine (CIRAC) et professeur émérite à l'université de Cergy-Pontoise

\section{Christophe LEININGER}

Directeur du développement des marchés, Commission de régulation de l'énergie (CRE), Paris

\section{Philipp LITZ}

Chargé de mission Lutte contre le réchauffement climatique, données et outils, Agora Energiewende, Berlin

\section{Hervé MIGNON}

Directeur Économie, Prospective et Transparence, Réseau de transport d'électricité (RTE), La Défense

\section{ROMain MOREL}

Chef de projet Finance, investissement et climat à I4CE (Institute for Climate Economics), Paris (jusqu'en 2016)

Actuellement Financial Modeling Expert, Groupe SNI (Groupe Caisse des Dépôts), Montpellier 


\section{Jürgen OSER}

Directeur du service Coopération transfrontalière et Affaires européennes (SGZE)

au Regierungspräsidium de Fribourg-en-Brisgau (Land de Bade-Wurtemberg)

\section{Thomas Pellerin-Carlin}

Chercheur à l'Institut Jacques Delors sur la politique européenne de l'énergie et la politique européenne de défense, Paris

\section{Jean-Claude PERRAUDIN}

Conseiller énergie atomique et énergies alternatives à l'ambassade de France en Allemagne,

Berlin (jusqu'en 2016)

Actuellement chargé des affaires publiques européennes au Commissariat à l'énergie atomique et aux énergies alternatives (CEA), Saclay

\section{Dimitri PESCIA}

Senior Associate au sein de l'équipe Coopération européenne en matière d'énergie, Agora Energiewende, Berlin

\section{Stéphane REICHE}

Conseiller économique Transport, énergie, environnement à l'ambassade de France en Allemagne, Berlin

\section{Sven RÖSNER}

Directeur de l'Office franco-allemand pour la transition énergétique (OFATE), Paris

\section{Anne TURFIN}

Chargée de mission Contrôle des émissions de $\mathrm{CO}_{2}$ et de la consommation d'énergie chez Klima-Bündnis à Francfort-sur-le-Main

\section{Wolfram VOGEL}

Directeur Affaires publiques et communication, European Power Exchange - EPEX SPOT, Paris

\section{Marek WASIŃSKI}

Expert pour le programme Relations économiques internationales et défis mondiaux à l'Institut polonais des Affaires étrangères (Polski Instytut Spraw Międzynarodowych, PISM) de Varsovie 
Composition : CIRAC http://www.cirac.u-cergy.fr CIRAC, c/o Université de Cergy-Pontoise

33, boulevard du Port - 95011 CERGY-PONTOISE CEDEX

Impression : Dupli-print

2, rue Descartes - Z.I. Sezac - 95330 DOMONT

Dépôt légal : juin 2017

$\mathrm{N}^{\circ}$ d'impression : 2017060110

Imprimé en France 